\title{
Low-Power Stlicon-based Thermal Sensors and Actuators for Chemical Applications
}

\section{Elizaveta Vereshchagina}
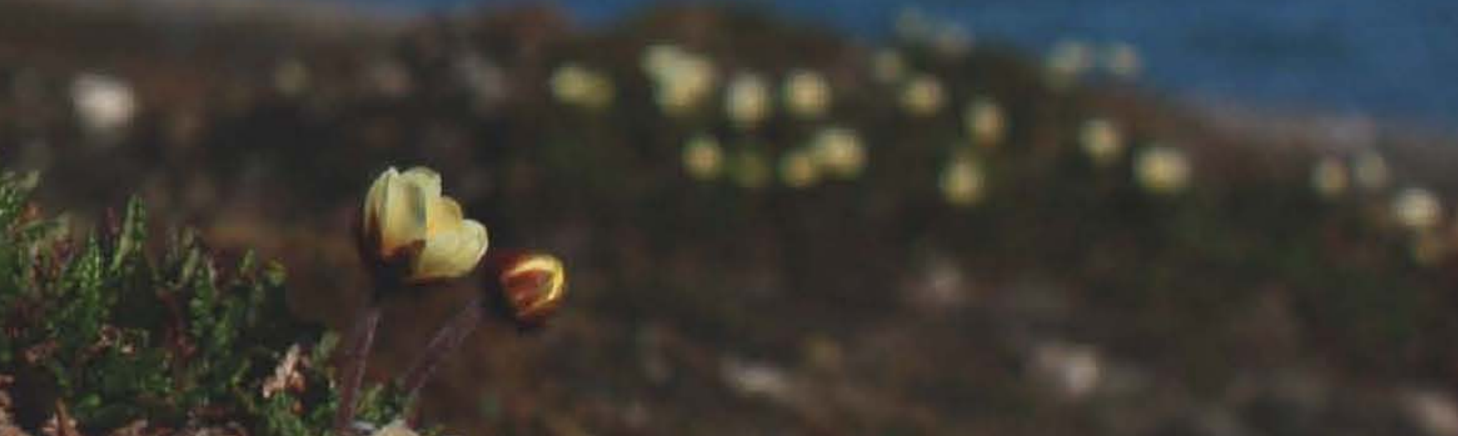

$1+3,-20$

y.

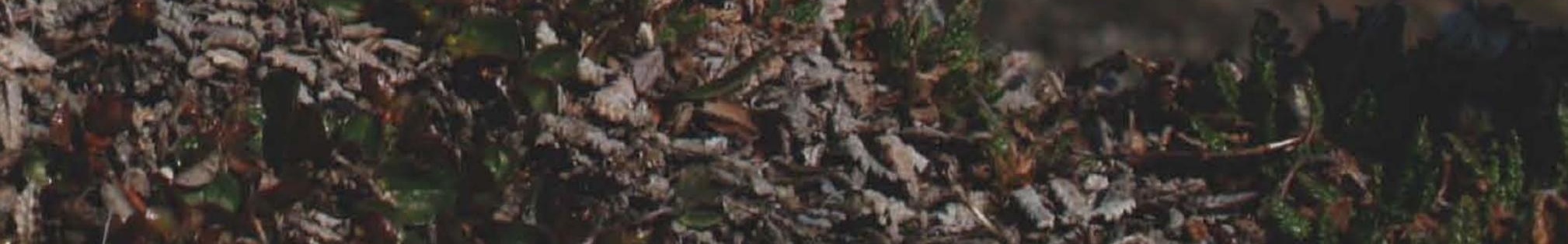




\section{LOW-POWER SILICON-BASED THERMAL SENSORS AND ACTUATORS FOR CHEMICAL APPLICATIONS}

Elizaveta Vereshchagina 


\section{Samenstelling promotiecommissie:}

Prof. dr. Gerard van der Steenhoven (voorzitter) Universiteit Twente

Prof. dr. J.G.E. Gardeniers (promotor)

Universiteit Twente

Prof. dr. K. Seshan

Universiteit Twente

Prof. dr. ing. A.J.H.M. Rijnders

Universiteit Twente

Prof. dr. R. Dekker

Technische Universiteit Delft

Dr. A.Y. Kovalgin

Universiteit Twente

Dr. A.G. van den Bos

Sensata Technologies

Prof. dr. R.A.M. Wolters

Universiteit Twente.

The work in this thesis was carried out in the Mesoscale Chemical Systems group, MESA+ Institute for Nanotechnology, University of Twente. It is part of the project: Super Low Power Hot Surface Silicon Devices for Chemical Sensors and Actuators financially supported by the Dutch Technology Foundation STW (Hot Silicon, nr. 07682).

Publisher:

Elizaveta Vereshchagina, Mesoscale Chemical Systems, University of Twente,

P.O. Box 217, 7500 AE Enschede, The Netherlands

mcs.tnw.utwente.nl

Cover design: David Oberholzer. The picture was taken on $11^{\text {th }}$ July 2009 in the northern part of Spitsbergen. The lake on the background is Lomfjord. The flower is Mountain avens.

Print: Gildeprint, Enschede, The Netherlands

(C) Elizaveta Vereshchagina, Enschede, The Netherlands 2011

No part of this work may be reproduced in any form

without the permission in writing from the publisher.

ISBN: 978-90-365-3300-3 


\title{
LOW-POWER SILICON-BASED THERMAL SENSORS AND ACTUATORS FOR CHEMICAL APPLICATIONS
}

\author{
PROEFSCHRIFT \\ ter verkrijging van \\ de graad van doctor aan de Universiteit Twente, \\ op gezag van de rector magnificus, \\ Prof. dr. H. Brinksma, \\ volgens besluit van het College voor Promoties \\ in het openbaar te verdedigen \\ op vrijdag 9 december 2011 om 16.45 uur \\ door
} Elizaveta Vereshchagina geboren op 16 Mai 1984 te Sint-Petersburg, Rusland 
Dit proefschrift is goedgekeurd door de promotor:

Prof. dr. J.G.E. Gardeniers 
To my family and Roman. 
1 Introduction $\quad 1$

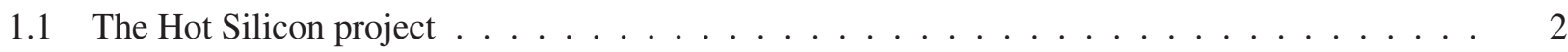

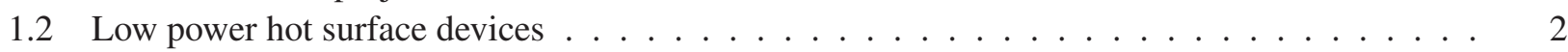

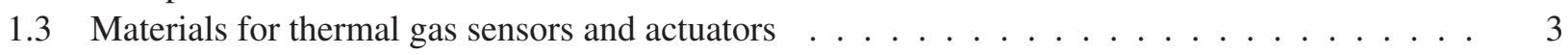

1.4 Micro- and nano- calorimeters $\ldots \ldots \ldots \ldots \ldots \ldots$

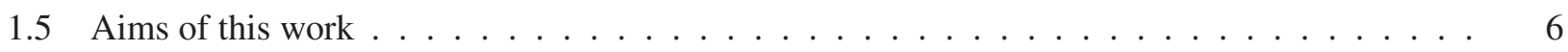

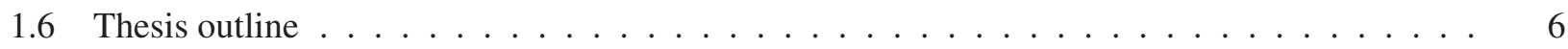

2 Thermo-chemical model for catalytic microsensors 9

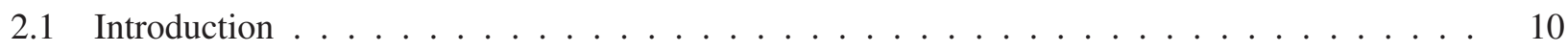

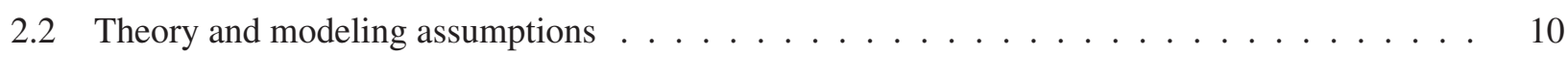

2.3 Results and discussion $\ldots \ldots \ldots \ldots \ldots \ldots \ldots \ldots$

2.4 Summary and conclusions $\ldots \ldots \ldots \ldots \ldots \ldots$

3 Measurement of reaction heats using a polysilicon-based microcalorimetric sensor 29

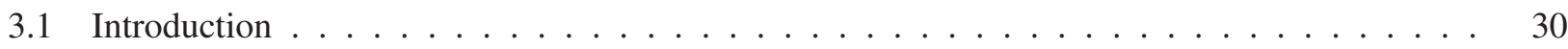

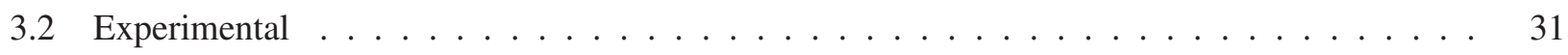

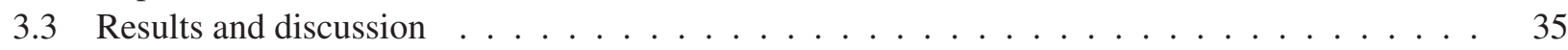

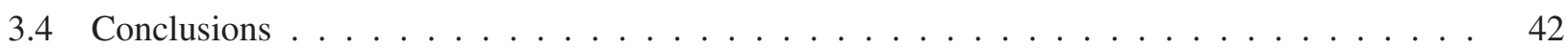

4 Development of titanium silicide - boron-doped polysilicon resistive temperature sensors 45

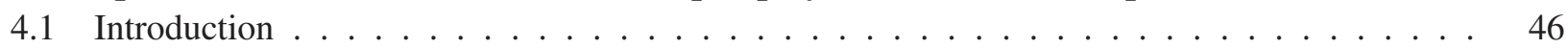

4.2 Choice of a material for temperature sensing . . . . . . . . . . . . . . . 46

4.3 Formation and characterization of the $\mathrm{TiSi}_{2}$ thin films . . . . . . . . . . . . . . . . 49

4.4 Fabrication of the temperature sensors and heaters . . . . . . . . . . . . . . . . 52

4.5 Results and discussion $\ldots \ldots \ldots \ldots \ldots \ldots \ldots$

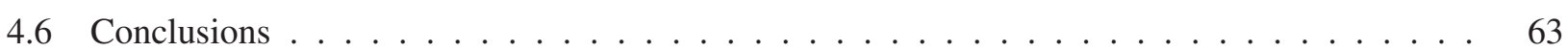

5 Characterization of the $\mathrm{TiSi}_{2} / \mathrm{Poly}-\mathrm{Si}$ micro hotplates for microcalorimetric analysis of gaseous $\begin{array}{lr}\text { samples. } & \mathbf{6 5}\end{array}$

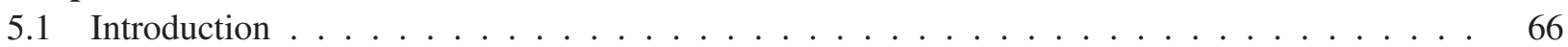

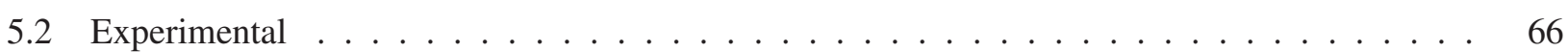

5.3 Results and Discussion $\ldots \ldots \ldots \ldots \ldots \ldots \ldots \ldots$

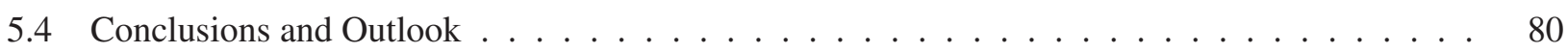

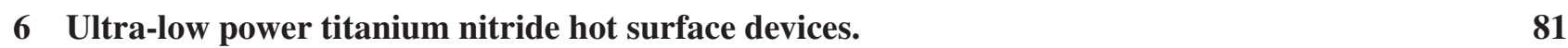

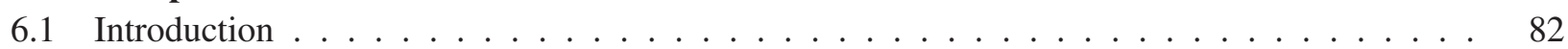

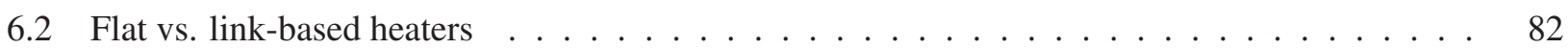

6.3 Overview of designs and fabrication $\ldots \ldots \ldots \ldots \ldots \ldots$

6.4 Selected applications $\ldots \ldots \ldots \ldots \ldots \ldots$

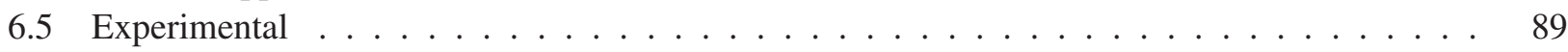

6.6 Results and discussion $\ldots \ldots \ldots \ldots \ldots \ldots \ldots$ 
6.7 Outlook and remarks $\ldots \ldots \ldots \ldots \ldots$

7 Selection, characterization and local deposition of catalytic thin films. 95

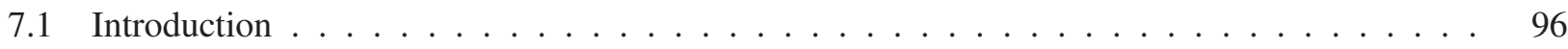

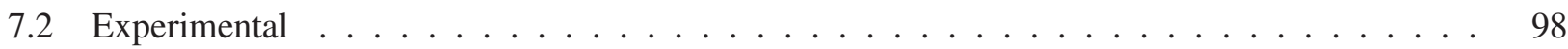

7.3 Results and Discussion . . . . . . . . . . . . . . . . . . . . 102

7.4 Summary and conclusion . . . . . . . . . . . . . . . . . . . . 109

8 Outlook $\quad 111$

8.1 Design and fabrication of hot surface devices $\ldots \ldots \ldots \ldots \ldots \ldots \ldots \ldots$

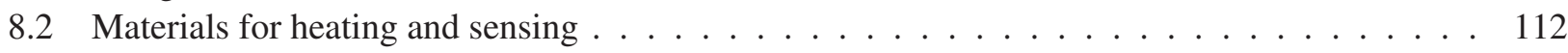

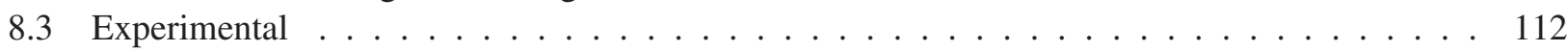

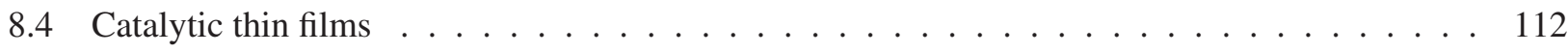

8.5 Nanolink-based devices . . . . . . . . . . . . . . . . . . . . . . 112

$\begin{array}{ll}\text { Appendix } & 123\end{array}$

$\begin{array}{ll}\text { Summary } & 125\end{array}$

$\begin{array}{ll}\text { Samenvatting } & 127\end{array}$

$\begin{array}{lr}\text { List of Publications } & 129\end{array}$

$\begin{array}{ll}\text { Acknowledgments } & 131\end{array}$

$\begin{array}{ll}\text { Author biography } & 135\end{array}$ 


\title{
List of Nomenclature
}

\author{
Roman \\ A Cross-sectional area, $\mathrm{m}^{2}$ \\ $B$ \\ Coefficient proportionality \\ Correction factor \\ $C_{p}$ \\ Heat capacity at constant pressure, $\mathrm{J} / \mathrm{kg} \cdot \mathrm{K}$ \\ Thermal capacitance, $\mathrm{W} / \mathrm{K}$ \\ Diffusion coefficient, $\mathrm{m}^{2} / \mathrm{s}$ \\ Density, $\mathrm{kg} / \mathrm{m}^{3}$ \\ Electric field intensity, $\mathrm{V} / \mathrm{m}$ \\ Activation energy, $\mathrm{J} / \mathrm{mol}$ \\ Accelerating energy of implanted ions, $\mathrm{J}$ \\ Standard enthalpy of formation, $\mathrm{J} / \mathrm{mol}$ \\ Electric current, A \\ Electric current density, $\mathrm{A} / \mathrm{m}^{2}$ \\ Heat, $\mathbf{J}$ \\ Heat source, $\mathrm{W} / \mathrm{m}_{3}$ \\ Resistance, p.d.u. \\ Reynolds number \\ Reaction term, $\mathrm{mol} / \mathrm{m}^{3} \cdot \mathrm{s}$ \\ Temperature, ${ }^{\circ} \mathrm{C}(\mathrm{K})$ \\ Electric potential, $\mathrm{V}$ \\ Volume, $\mathrm{m}^{3}$ \\ Conversion \\ Area, $\mathrm{m}^{2}$ \\ Concentration, $\mathrm{mol} / \mathrm{m}^{3}$ \\ Distance in $\mathrm{x}, \mathrm{y}$, or $\mathrm{z}$ direction, $\mathrm{m}$ \\ Heat transfer coefficient, $\mathrm{W} / \mathrm{m}^{2} \mathrm{~K}$ \\ Pre-exponential (frequency) factor, $\mathrm{mol} /\left(\mathrm{kg}_{\mathrm{cat}} \cdot \mathrm{s} \cdot \mathrm{Pa}\right)$ \\ Length, $\mathrm{m}$ \\ Mass, kg \\ Pressure, $\mathrm{Pa}$ \\ Reaction rate, $\mathrm{Pa}$ \\ Heat flux, $\mathrm{J} / \mathrm{m}^{2} \cdot \mathrm{s}$ \\ Thickness, $\mathrm{m}$ \\ Gas velocity, $\mathrm{m} / \mathrm{s}$ \\ Width, $\mathrm{m}$
}

\section{Greek}

$\beta$

Temperature coefficient of resistance, $\mathrm{K}^{-1}$ Fitting parameter: slope of the $\mathrm{R}(\mathrm{T})$ curve

$\varepsilon_{g}$

Porosity, vol\% 


\section{Index}

air

Al

$a m b$

av

cav

ch

cond

conv

dif

fall

flux

flead

gas

$h$

heat

in

lin

$\max$

meas

memb

min

out

ox

reac

res

rise

$s$

Si

slead

sol

src

sup

surf

th

tot

Abbreviations

AFM

BET

CMOS

CVD

DC

GC
Thermal time constant, sec

Thermal conductivity, $\mathrm{W} / \mathrm{m} \cdot \mathrm{K}$

Resistivity, $\Omega \cdot \mathrm{m}$

Electric conductivity, $\mathrm{S} / \mathrm{m}$

Tortuosity

Air

Alumina support

Ambient

Average

Cavity etched in silicon substrate

Channel

Conduction

Convection

Diffusion

Fall thermal time constant

Heat flux to ambient

Force connection lead

Gaseous media

Heater

Heat source

Inlet

Linear

Maximum

Measured

Thermally isolating membrane

Minimum

Outlet

Oxidation

Reaction

Resistive

Rise thermal time constant

Sheet

Silicon substrate

Sense connection lead

Solid

Source

Supplied

Surface

Thermal

Total

Atomic Force Microscope

Brunauer-Emmett-Teller Method

Complementary Metal-Oxide-Semiconductor

Chemical Vapor Deposition

Direct Current

Gas Chromatograph 
GND

IV

HRSEM

LEL

LPCVD

MEMS

MIMIC

$\mu \mathrm{CP}$

$\mu \mathrm{TM}$

PDMS

PECVD

PFDTS

RIE

SEM

TCR

TEM

XPS

XRD

\section{Constants}

$R_{i d}$

$k$
Ground

Current-Voltage characteristic

High-Resolution Scanning Electron Microscope

Low Explosion Limit

Low Pressure Chemical Vapor Deposition

Microelectromechanical Systems

Micromolding in Capillaries

Micro Contact Printing

Micro Transfer Molding

Polydimethylsiloxane

Plasma Enhanced Chemical Vapor Deposition

Perfluorodecyltrichlorosilane

Reactive Ion Etching

Scanning Electron Microscope

Temperature Coefficient of Resistance

Transmission Electron Microscope

X-ray Photoelectron Spectroscopy

$\mathrm{X}$-ray Diffraction

Ideal gas constant, $8.31 \mathrm{~J} / \mathrm{mol} \cdot \mathrm{K}$

Boltzmann constant, $1.38 \cdot 10^{-23} \mathrm{~J} / \mathrm{K}$ 


\section{1 \\ Introduction}

In this chapter the background and goals of the Hot Silicon project are introduced: terminology, definitions and state-of-the-art overviews of a series of devices and applications are given, as well as a detailed description of the content of each chapter. 


\subsection{The Hot Silicon project}

The work described in this thesis is done within the framework of the "Hot Silicon" project. The official name of this project is "Super Low Power Hot-Surface Silicon Devices for Chemical Sensors and Actuators". The project is carried out within the STW ("Stichting Technische Wetenschappen" - Dutch Technology Foundation) framework under the project number 07682.

The "Hot Silicon" project is a cooperation between the research chairs Mesoscale Chemical Systems (MCS) and Semiconductor Components (SC), the MESA+ Institute for Nanotechnology of the University of Twente (the Netherlands). In this project we study the feasibility of ultra-low-power and CMOS compatible hot surface micro devices and their application as chemical sensors and actuators.

\subsection{Low power hot surface devices}

Hot surface devices, also referred to as micro hotplates, are transducer elements that include functionality for thermal actuation and temperature measurement (and control), and are suitable for monitoring temperaturedependent physical and/or chemical phenomena on microscale [1]. A general concept of the micro hotplate device consists of heaters, temperature sensors and, in some cases, a specific chemical interface. Due to their high thermal sensitivity, rapid heating and cooling, micro hotplates have been used widely for a variety of sensing applications, including:

- Flow sensor [2],

- Thermal conductivity detector [3], [4],

- Adsorptive metal-oxide-based sensor [5]-[7]

- Infrared bolometer [8],

- Catalytic sensor (micro combustor) [9]-[13].

Some of these applications operate on calorimetric principle, i.e. involve measurement of temperature change, described in detail in Chapter 5.

The efficiency of heat generation $\left({ }^{\circ} \mathrm{C} / \mathrm{mW}\right)$, thermal sensitivity $\left(/{ }^{\circ} \mathrm{C}\right)$ and surface temperature distribution are major criteria for determining feasible sensor applications.

A higher thermal resistance of the hot surface device in a particular ambient increases the thermal sensitivity. As a result, a thin isolating membrane and suspended micro bridges have become an unavoidable solution in current low-power micro hotplate fabrication technology [14]-[17]. The main idea is to use a "release etch" of the silicon substrate as a final fabrication step, in order to suspend the active area of the sensor on a dielectric membrane or beams. This restricts heat conduction to the substrate, one of the main mechanisms of thermal losses, and allows detection of small temperature variation at decreased levels of power consumption. The power consumption of most of the state-of-the-art micro hotplates is in the range 20-50 $\mathrm{mW}$ [5]-[13].

Sensitivity to heat effects is determined by the temperature transduction mechanism (temperature sensitive resistor, thermocouple, transistor, diode) and the measurement method used for the readout of the sensor output. Nowadays thermal sensors allow the measurement of temperature changes with a resolution down to $10^{-4}{ }^{\circ} \mathrm{C}$. A further reduction in power consumption and an increase in the thermal sensitivity can be achieved by implementation of new approaches to the design of micro hot plate devices, such as concepts of physical amplification, i.e. downscaling of the dimensions of heater and temperature sensor and implementation of alternative resistive materials.

Downscaling of hot surface devices is an important step towards accurate and rapid measurement of the temperature at the low micro- and nanoscale range. Besides enhanced sensitivity and high spacial resolution that can be achieved in nanoscale thermal sensors [18], fabrication of such miniaturized hot surface devices with 
standard CMOS technology allows a further upgrade to portable silicon-based sensing systems as well as addition of electronic control on a single chip.

Thus, on one hand, the ultra-low power consumption and high sensitivity can be achieved by minimization of the dimensions of the sensor and actuator, on the other hand, this can also be accomplished by implementation of an alternative concept of heat dissipation and measurement.

In literature, two examples of ultra-low-power (below $3 \mathrm{~mW}$ ) chemical sensors and actuators, so called "suspended membrane actuators" (SMAs), employing the idea of heat dissipation on low micro- and nanoscale, are demonstrated [19], [20]. Device operation is based on heat generation inside a highly-ohmic conductive nanolink, $10-100 \mathrm{~nm}$ in diameter, acting simultaneously as a heater and as a temperature sensor. A distinctive difference between nanolink-based and state-of-the-art (suspended flat heater) hot surface devices is that heat is generated locally (inside the link with defined geometrical dimensions) and that thermal losses through the leads are maximally suppressed.

When made using a reproducible fabrication routine, ultra-low power hot surface devices can become valuable transducers for low-power, simple, cheap chemical sensors and actuators. Additionally, due to the minimal power requirements the ultra-low-power hot surface devices can be easily coupled with a wireless transmission device for portable sensor systems at remote locations.

\subsection{Materials for thermal gas sensors and actuators}

For microcalorimetric gas sensing applications, the thermal sensor and actuator needs to be integrated in a micro flow channel and has to be partially covered with a catalyst to facilitate the high-temperature detection of oxidation reaction.

The concept of an ultra-low-power thermal sensor and actuator is based on the behavior of a highly-ohmic micro- or nano- link that can be operated simultaneously as a heater and as a temperature sensor. When using more than one resistive structures, i.e. link-based or flat thin film resistors, the actuating and sensing functions can also be separated. Originating from this concept and due to the technological and operational requirements imposed by the application, the focus of this study is on resistive methods for temperature sensing. The resolution and operating temperature of commonly used temperature sensors is demonstrated in Figure 1.1. Among

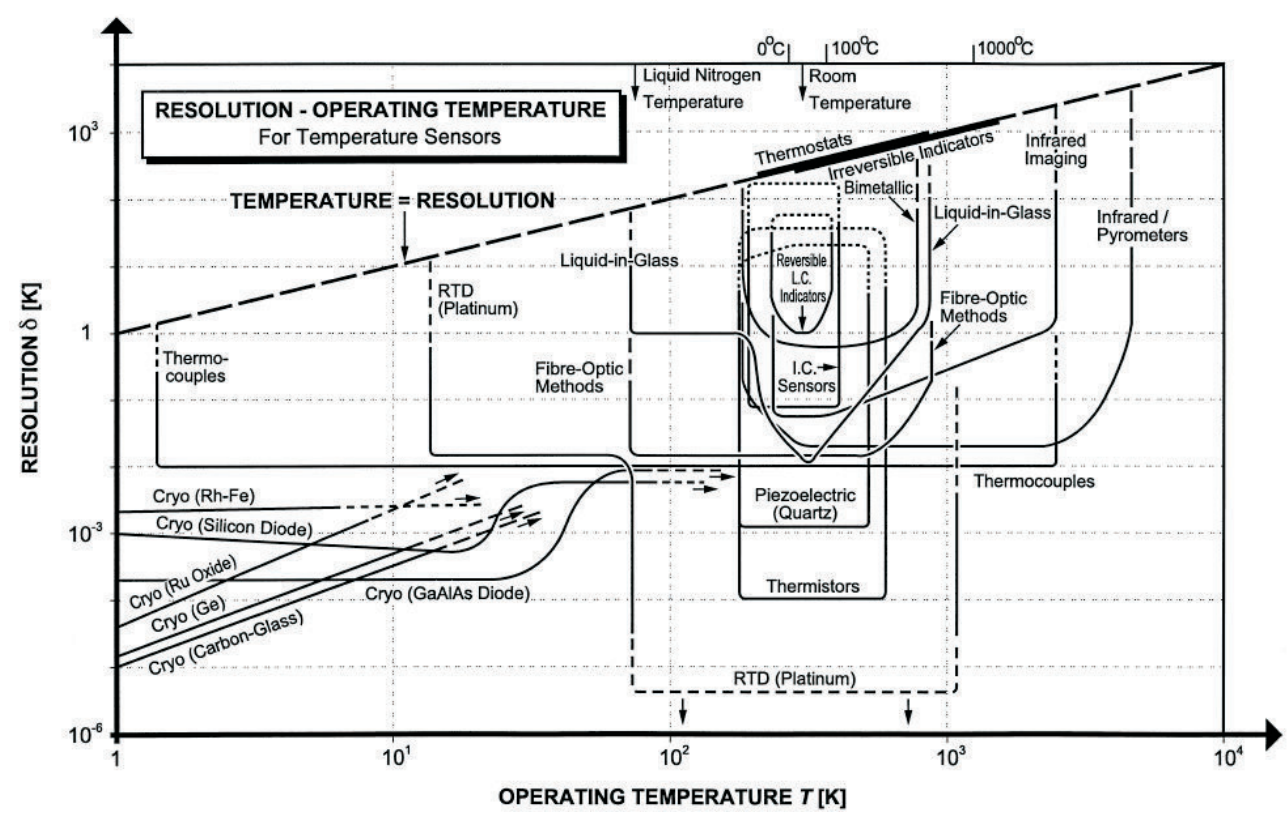

Figure 1.1: Resolution versus operating temperature for temperature sensors [22].

a variety of thermal CMOS-based sensors [21] thermocouples are the best candidates in terms of the tempera- 
ture resolution and operating temperature range [22]. However, integration of thermocouples is technologically complex and requires complicated calibration compared to temperature sensitive resistors.

The control of the thermo-electrical properties of the selected resistive material is crucial.

A requirement of CMOS compatibility of technological processes [23] imposes strict constraints on the choice of resistive materials. If the resistive temperature sensors are intended for integration in micro- and nano- calorimetric sensing systems, a high and stable temperature coefficient of resistance (TCR) and stable operation at elevated temperatures and in high oxidizing environment are necessary.

Three types of thin film resistive materials were used for thermal sensing and actuation throughout this work:

1. Boron doped polycrystalline silicon (poly-Si) [24]-[27];

2. A combination of poly-Si and titanium silicide [28];

3. Titanium nitride [29], [30].

These materials were selected for heating and temperature sensing applications based on their high resistivity, high temperature coefficient of resistance, compatibility with silicon processing and stability at elevated temperatures [31], [32], [33].

\subsection{Micro- and nano- calorimeters}

Among the variety of applications of micromachined hot surface devices, micro- and nano- calorimetry is an attractive concept [34], [35]. The concept of chemical sensing involves the measurement of heat generated by
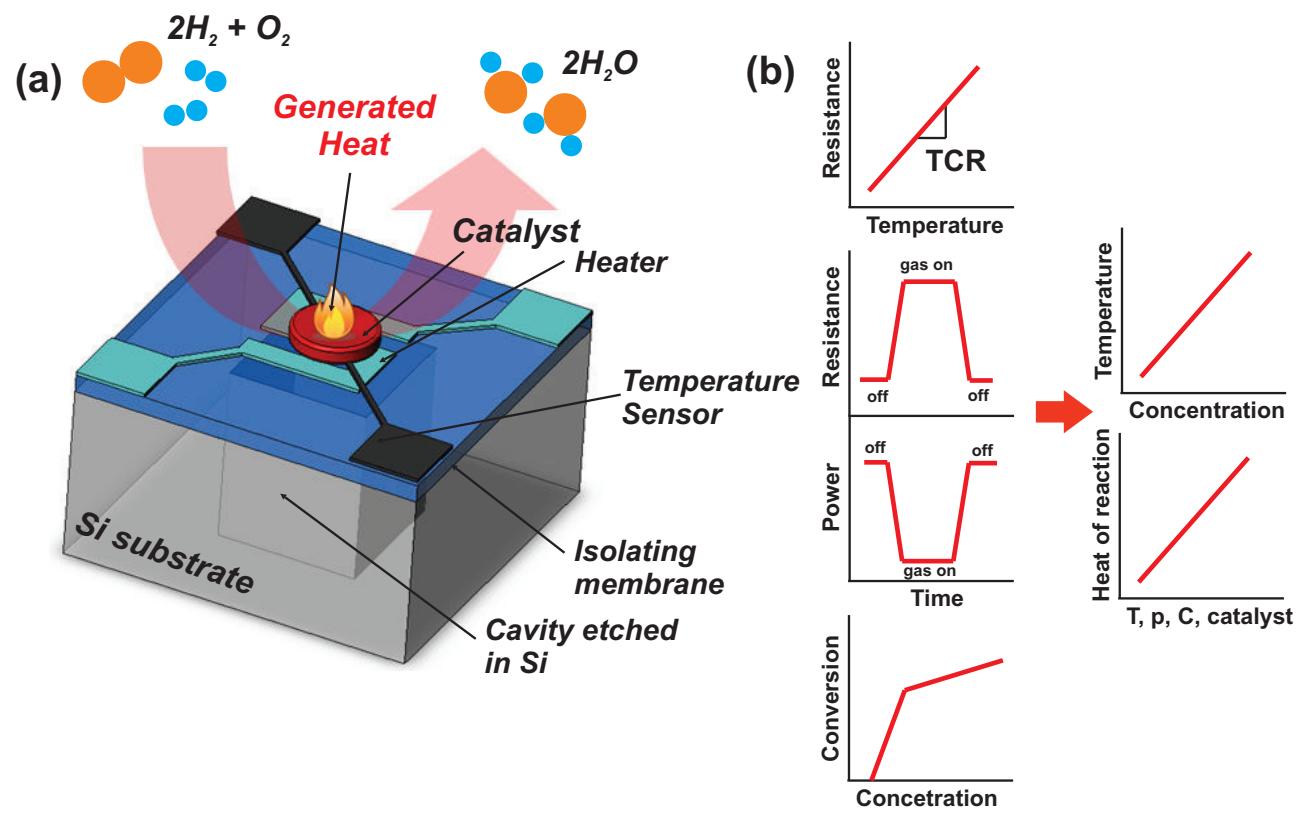

Figure 1.2: Schematic representation of a micro calorimetric sensor (a) and operational principle (b).

a chemical reaction or adsorption of a specific component on a reactive surface of the micro sensor (see Figure 1.2). The heat generation is determined by the measurement of the temperature change of resistive element(s), which is in direct contact with the reactive surface or the gas compartment [36], [37]. Microcalorimetry is a powerful technique and it provides a direct measure of the reaction heat effect. Microcalorimetric sensing has a long history, showing a continuous decrease in power consumption (see Figure 1.3): starting from the first Pt wire-based systems with a power consumption up to $1000 \mathrm{~mW}$ [38]-[40], to chip-based sensors consuming power in the range 30-200 $\mathrm{mW}$ [41]-[47], to low [12], [17] and ultra-low power, rapid, sensitive thermal sensors 
[19], [20] that require 1-30 $\mathrm{mW}$. The size of the sensors diminished to the nanometer scale, making ultrasensitive detection feasible at power levels below a few milliwatt. The application of this technique is very broad, and ranges from sensors for measurement of thermal properties of solids [48], gases [49], [50] to liquid bio-medical applications [51]-[54]. The physical measurable output of the micro- or nano- calorimeter is a temperature change. This change can be an indication of several physical and chemical phenomena at once: surface interaction (catalytic sensors), convective heat losses (flow sensors), thermal properties of the fluid (thermal conductivity detector), etc. For some applications this is an advantage, but also a challenge, as a sensor comprising only one resistive element, can not distinguish between heat produced by the reaction or by other (undesirable) sources such as phase changes or any other degradation in the catalytic or resistor materials, side reactions, ambient. The differentiation between these various phenomena causing alteration of the sensor

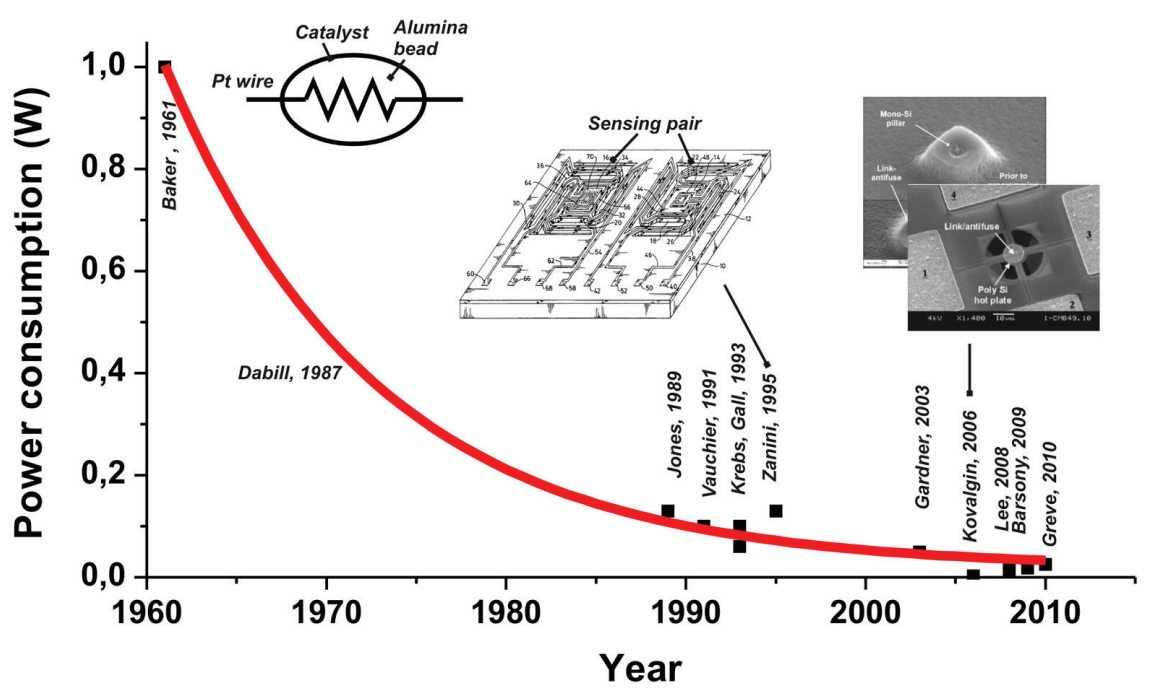

Figure 1.3: Evolution of microcalorimetric sensors.

output is achieved by e.g. combining the sensors in an array. A micro- or nanocalorimeter based on one resistive element, although sensitive to various thermally dependent phenomena, is never suitable as an ideal sensor for each of them simultaneously. However, by choosing an application specific design and measurement circuit, the desired thermal effects can be detected. When the output is calibrated with respect to the gas concentration, the sensor can be used for the measurement of a gas concentration. Under specific measurement conditions, the rate of the catalytic reaction depends on the concentration of the target gas, oxygen supply and actuating temperature. Determination of the reaction heat generated for a specific volume of gas is possible when the associated enthalpies, as well as the degree of conversion are known. Since this data is not always available, calibration of the sensor for a range of concentrations under similar conditions to the real application is required. Calibration also helps to circumvent possible sources of heat loss and parasitic influences, which may be very difficult to model a priori.

The selectivity of the catalytic microsensor highly depends on the choice of the chemical interface on which the gas species have a specific interaction, such as a catalytic surface reaction. The sensitivity is determined by the properties of the solid catalyst (activity), thermal sensitivity of the temperature transducer and the measurement method.

Various combustible gases in an oxidizing environment can be detected: hydrogen, carbon monoxide, methane, ethane, propane, etc. Examples of commercial microcalorimetric gas sensors are shown in Figure 1.4. 

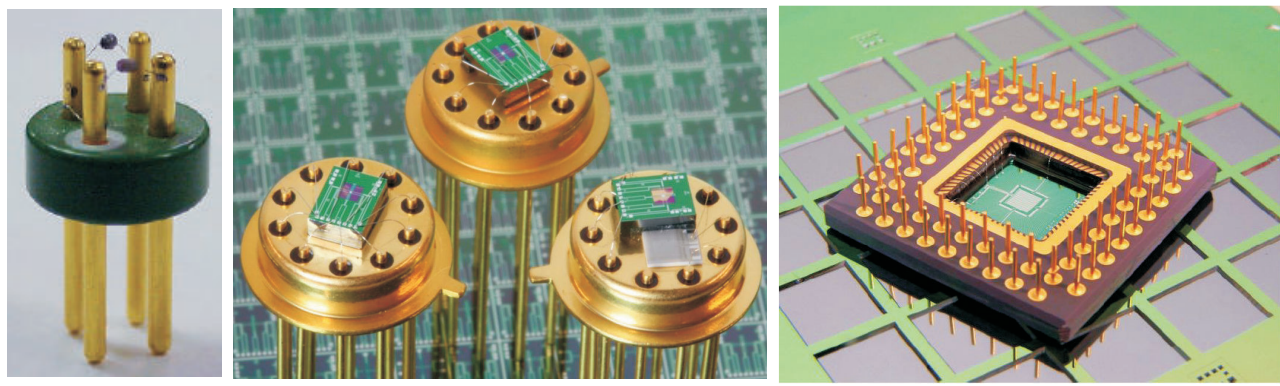

Figure 1.4: Catalytic bead sensor (a), Xensor gas microcalorimetric sensors (b, $c$ ).

\subsection{Aims of this work}

In the Hot Silicon project we aim to develop low and ultra-low-power Si-based hot surface devices, i.e. thermal sensors and actuators, for an application as catalytic gas micro sensors, micro- and nano- calorimeters.

Within the "Hot Silicon" framework the objectives of this work are:

1. Design and fabrication of the suspended membrane ultra-low-power hot-surface Si-based thermal sensors and actuators,

2. Integration of the sensors and actuators into a micro flow channel, and investigation of the related microfluidic aspects, i.e. flow behavior and control, heat transport, transport of reactive species,

3. Investigation of potential applications in the field of micro calorimetric sensing, i.e. studying thermallyactivated physical and chemical processes on-a-chip, i.e. detection of reaction heats, adsorption/desorption kinetics.

In microcalorimetric gas sensors the high temperature oxidation of hydrocarbons is initiated on the sensor and the heats released as a result of the reactions need to be accurately detected. This imposes several requirements to the sensor:

1. Reliable operation in the range $100-600{ }^{\circ} \mathrm{C}$ required for detection of combustible gases;

2. Low power operation which is essential for the future integration;

3. Reliable operation in a highly oxidizing environment;

4. Chemical inertness of the materials comprising the sensor.

\subsection{Thesis outline}

Chapter 2 describes the results of finite element analysis of a catalytic micro flow system consisting of a micro hotplate at elevated temperature with a catalyst. The influence of the design and material parameters on the power consumption, maximum operating temperature and surface temperature distribution is analyzed, and the results are presented. Furthermore, issues related to downscaling of the heater and a correlation between the thermal design of the micro hotplate and conditions of the catalytic reaction are discussed.

In Chapter 3 a low-cost, low-power, small sample volume microcalorimetric sensor for measurement of reaction heats is presented. The detection of reaction heats for a model reaction - the catalytic oxidation of propane in air - at concentrations of propane 0.01 to $0.8 \mathrm{vol} \%$ is demonstrated. Thermo-electrical properties of the polysilicon as a sensor material are discussed. 
Chapter 4 discusses the fabrication method, morphological and electrical characterization of the TiSi $2 / \mathrm{poly}_{2} \mathrm{Si}$ thin film temperature sensors to be implemented in hot surface micro devices. The temperature sensors exhibited a high temperature coefficient of resistance (TCR) of $3.8 \times 10^{-3} /{ }^{\circ} \mathrm{C}$, a linear dependence of resistance on temperature and an excellent thermal and electrical stability up to $800{ }^{\circ} \mathrm{C}$. The thermo-electrical characterization and sensing performance of such $\mathrm{TiSi}_{2} /$ poly-Si based hot surface devices are presented in Chapter $\mathbf{5}$

In Chapter 6 platinum (Pt) and palladium (Pd), both as sputtered thin film and as nanoparticles supported on $\gamma$-alumina, are compared in terms of their applicability as functional coatings in catalytic gas sensors. The deposition techniques applied and their limitations are discussed.

In Chapter 7 the feasibility of ultra low power titanium nitride link-based devices as resistive sensors and actuators is described. The devices can facilitate local heating and temperature sensing up to $300{ }^{\circ} \mathrm{C}$ at a power consumption less than $3 \mathrm{~mW}$, and for particular designs even down to a few $\mu \mathrm{W}$. This enables potential applications in various portable sensing systems, where rapid temperature sensing and/or thermal actuation with high spacial resolution and minimized power consumption are required.

Finally, in Chapter $\mathbf{8}$ a summary of the results obtained is given, followed by recommendations for future research. 


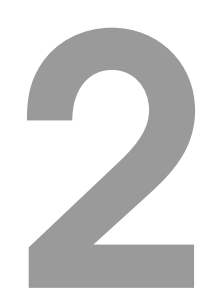

\section{Thermo-chemical model for catalytic microsensors}

In this chapter we study catalytic micro flow systems consisting of a micro hotplate and a deposited reactive catalyst at elevated temperature. Successful operation of such a system as a thermal actuator for chemical reaction and thermal sensor for the evolved reaction heats requires a thermally balanced design of the heater and controlled reaction and flow conditions. Understanding of these factors helps to develop application specific designs.

For this purpose a model was developed including ( $i$ ) 3D thermal model of the suspended heater and (ii) $2 D$ model of thermally actuated chemical reaction in the flow channel. The calculations were performed using Comsol Multiphysics 4.2 software.

The influence of the design and material parameters of the micro hotplate on the power consumption, the maximum achievable temperature and the surface temperature distribution is analyzed and the results are presented. Specifically, we discuss issues related to downscaling of the heater and establish a correlation between the thermal design of the micro hotplate and reaction conditions. 


\subsection{Introduction}

\subsubsection{Motivation}

Thermal actuation is a process in which physical or chemical processes are initiated by means of introduction of heat to, for example, the surface of the micro hotplate [55]. Micro-hotplates are devices used to maintain locally high temperatures, typically up to 500-650 K and, for particular applications, even beyond [56]. Micro-hotplates commonly utilize a thermally isolating membrane to improve the heat confinement and, as a consequence, thermal sensitivity to evolved heat. General concepts for their design aim at the reduction of power consumption, fast heating and cooling rates, and the uniformity of temperature [57] in the heated area. The former is important for portable chemical sensors [58] where energy consumption is critical and the latter two for kinetic studies [59], [60] and catalyst screening [61].

The development of a good model for thermo-chemical actuation and possible heat loss mechanisms is crucial for the design of various catalytic microsystems, e.g. microcalorimetric sensors [62], [63], microfluidic calorimeters [64] and catalytic microreactors [65], [66]. Such a model allows to reduce the amount of labbench experiments and to predict device performance with respect to a specific application.

Although the approaches to micro hotplate optimization are established and were demonstrated in the past for adsorptive gas sensors and flow meters [67], [68], there is a lack of information on their optimization in combination with the catalytic reaction carried out inside of a flow channel. Heat and mass transfer aspects in a heated micro-channel [69] and combustor [70] system were investigated on mesoscale level independently.

The goal of this study is to identify the main mechanisms influencing the thermal efficiency (power supplied with respect to heat generated) and thermal sensitivity of catalytic micro hotplate flow systems and to develop an approach for their optimization in relation to the catalyst properties and reaction conditions.

\subsubsection{Optimization parameters}

In evaluating the micro hotplate design the following factors are important:

- Minimized power consumption (reduced heat losses);

- Thermal efficiency, i.e. maximum temperature on the membrane at a required level of power consumption;

- Uniform surface temperature distribution;

- Fast heating and cooling times.

When integrating in a flow channel for catalytic studies on a chip the following factors are important to consider with respect to generation of sufficient amount of heat to be detected:

- Volume of channel;

- Flow rate;

- Reaction temperature;

- Concentration of reacting species;

- Catalytic activity and possibly selectivity of an applied catalyst.

\subsection{Theory and modeling assumptions}

In this section model assumptions and theoretical background for the model are described. The selected geometry and boundary conditions are explained, followed by a description of multi-physical phenomena included in the model. 


\subsubsection{D micro hotplate}

\section{Geometry, materials and boundary conditions}

The selected 3D geometry is shown in Figure 2.1. A Si wafer, a thermal isolating membrane $\left(\mathrm{Si}_{3} \mathrm{~N}_{4}, \mathrm{SiO}_{2}\right.$ or $\mathrm{SiRN}$ ) and a meander-shaped poly-Si heater placed in a channel of a defined height. The dimensions corresponding to the geometry are summarized in Table 2.1. The heater was in a 4-point configuration, so that the

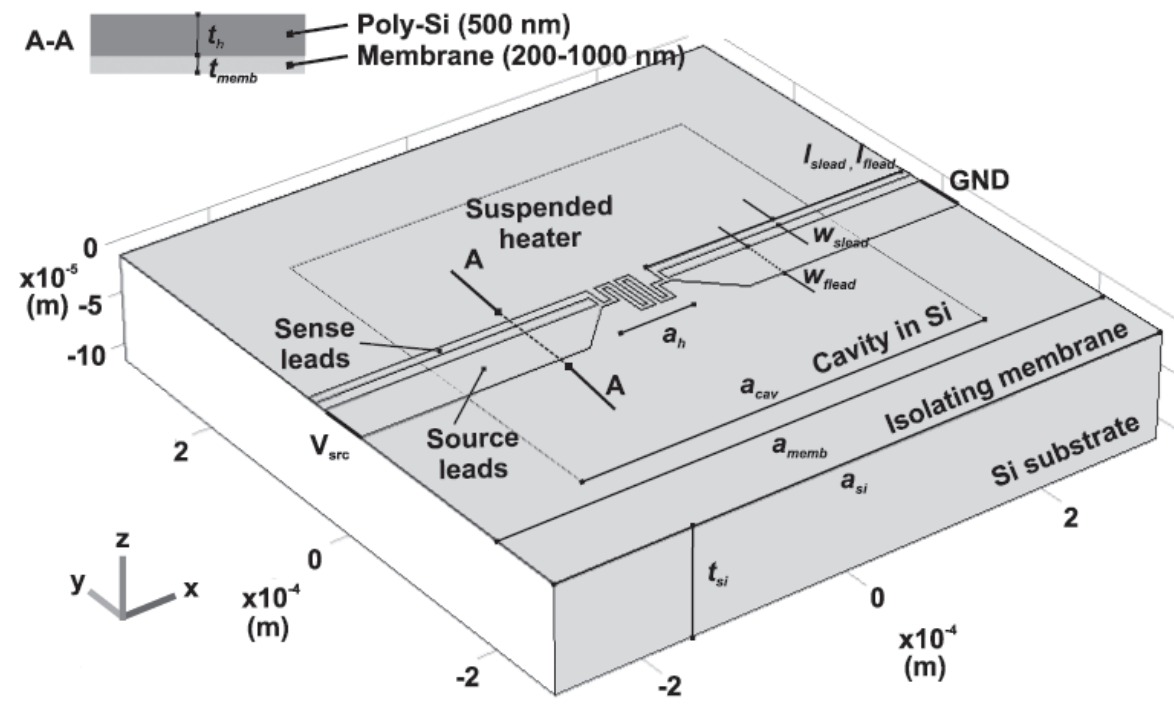

Figure 2.1: $3 D$ geometry of the micro-hotplate and corresponding cross sectional view A-A showing a heater suspended on a membrane.

Table 2.1: Dimensions used in geometry of the heater.

\begin{tabular}{lc}
\hline Parameter & Value $(\mu \mathrm{m})$ \\
\hline \hline $\mathrm{a}_{\text {cav }}$ & 400 \\
$\mathrm{a}_{h}$ & 50 \\
$\mathrm{a}_{\text {memb }}$ & 600 \\
$\mathrm{a}_{\text {si }}$ & 600 \\
$\mathrm{l}_{\text {flead }}$ & 220 \\
$\mathrm{l}_{\text {slead }}$ & 220 \\
$\mathrm{t}_{\text {ch }}$ & 200 \\
$\mathrm{t}_{h}$ & 0.5 \\
$\mathrm{t}_{\text {memb }}$ & 1 \\
$\mathrm{t}_{\text {si }}$ & 100 \\
$\mathrm{w}_{\text {flead }}$ & 50 \\
$\mathrm{w}_{h}$ & 5 \\
$\mathrm{w}_{\text {slead }}$ & 10 \\
\hline
\end{tabular}

heat losses through the connection leads are included into the model. The heater is assumed to act as a resistor, i.e. generate Joule heat. The complete geometry is electrically insulated except the leads, at which electrical potential and grounding are applied. To simplify the structure and reduce the simulation time, the thickness of the Si substrate was assumed to be $100 \mu \mathrm{m}$. However, in practice, the substrate has a larger thermal mass than used in our geometry. This design corresponds to micro hotplates we have studied previously [71], [72].

The mesh used in the numerical model was determined by the dimensions of the thin isolating membrane as it 
is the thinnest layer in the system. The materials and their thermo-physical data at $298 \mathrm{~K}$ are summarized in Table 2.2. The properties of poly-Si are selected equal to mono crystalline substrate. However, the real values are dependent on structural properties of poly-Si and on the dopant type and concentration [73], [74].

The ambient temperature, $T_{a m b}$ is assumed to be $293 \mathrm{~K}$. There is a normal heat flux $(q)$ present to the ambient. Radiation has been evaluated to be negligible within the operating range (from room temperature to $550 \mathrm{~K}$ ).

\section{Joule heating}

In this section the electrostatic behavior of the conductive poly-Si heater is defined. The heater is subjected to a potential difference $(\Delta U)$ which induces a current through the leads $(I)$ in the high resistive central part of the structure. The generated heat $(Q)$ is proportional to the square of the magnitude of the electric current density $(J)$, which was extracted from modeling results:

$$
Q=\frac{1}{\sigma}|J|^{2}=\frac{1}{\sigma}|\sigma \cdot E|^{2}=\sigma|\cdot \nabla U|^{2}
$$

The electrical conductivity $(\sigma)$ of the heater decreases with the temperature $(T)$ upon heating according to
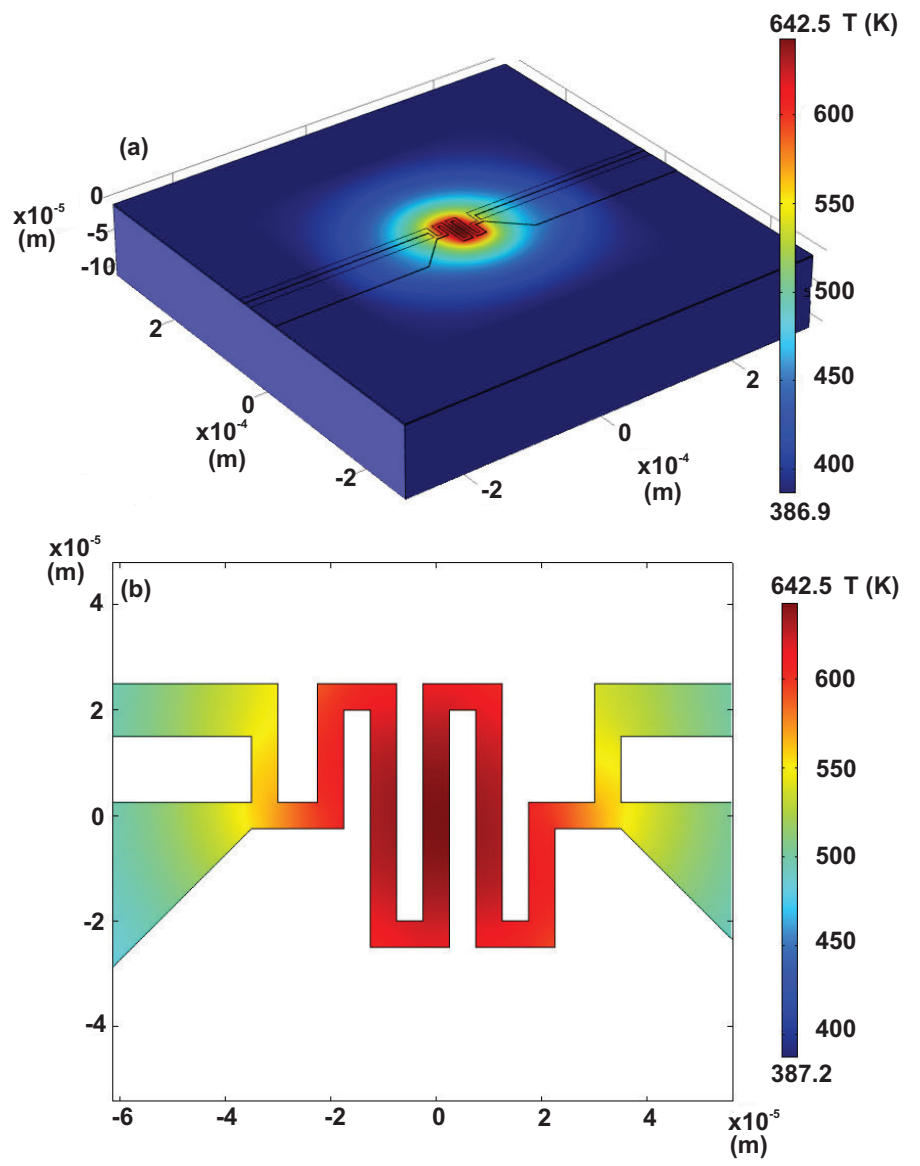

Figure 2.2: Temperature profile in $K$ along the $50 \times 50 \mu \mathrm{m}^{2}$ heater suspended on a $\mathrm{Si}_{3} \mathrm{~N}_{4}$ membrane of 1000 $n m$ thickness with a $400 \times 400 \mu \mathrm{m}^{2}$ cavity etched in the Si underneath the membrane. The power supplied to the heater is $14 \mathrm{~mW}$.

Equation 2.2 with the temperature coefficient of resistance $(\alpha)$, taken as $2.5 \times 10^{-4} / \mathrm{K}$ (this TCR was measured for the poly-Si extrinsically doped with boron [71]:

$$
\sigma=\frac{1}{\rho_{0} \cdot\left(1+\alpha \cdot\left(T-T_{a m b}\right)\right)}
$$




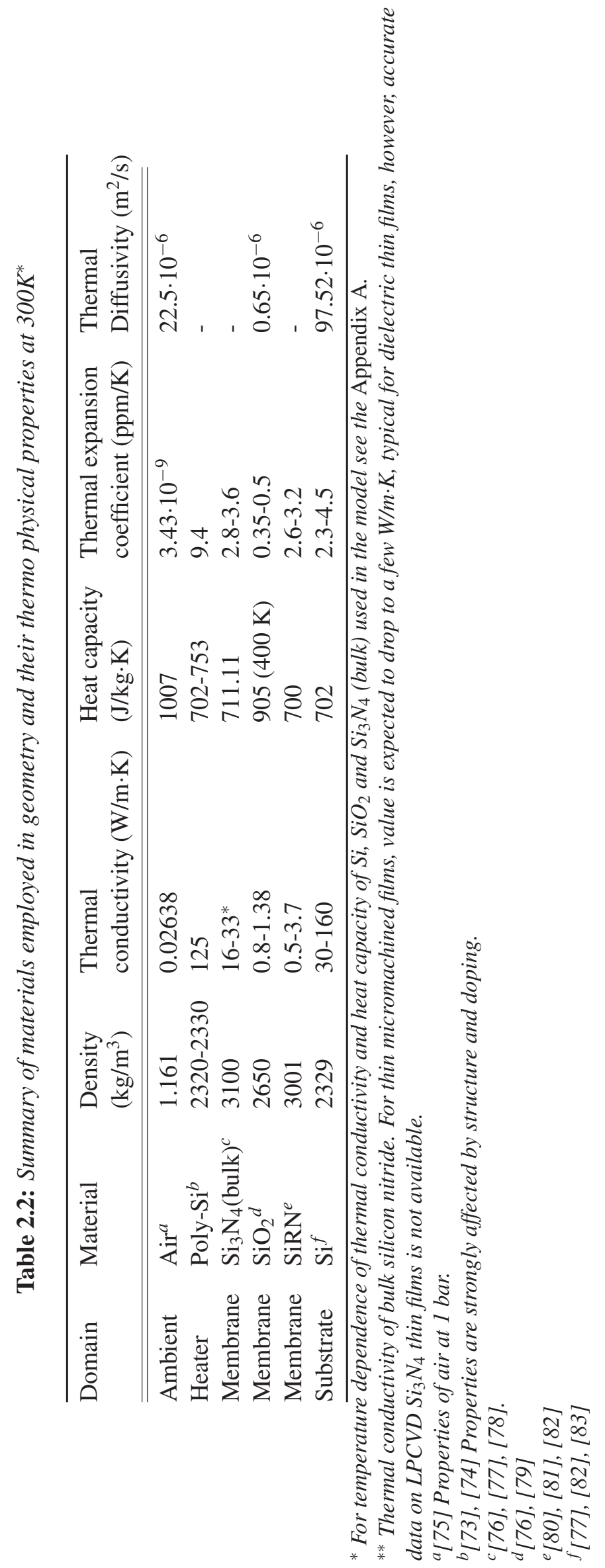




\section{Heat transfer}

The temperature of the heater rises when an electrical current passes through it due to electrical resistance, i.e. Joule heating. In Figure 2.2 the temperature profile of the 3D suspended heater (a) as well as a 2D surface temperature profile of the heater (b) are shown. The heat generated is transferred to the Si frame via the thermally isolating membrane according to Fourier's law for heat conduction. The amount of heat transferred per unit time $(\dot{Q})$ through cross-sectional area $(A)$ is proportional to thermal conductivity $(\lambda)$ :

$$
\dot{Q}=\frac{\lambda}{\Delta x} \cdot A \cdot \Delta T
$$

The temperature dependence of the thermo-physical properties, i.e. the thermal conductivity $(\lambda)$ and the specific heat capacity $\left(C_{p}\right)$, were incorporated in the model as a third order polynomial fit of the data available in the literature [76], [77], [83] (see Appendix A). The heat flux to the ambient $\left(q_{\text {flux-amb }}\right)$ from the hot surface is defined in terms of the heat transfer coefficient $\left(h_{\text {flux-amb }}\right)$ :

$$
\begin{gathered}
q_{\text {flux }-a m b}=h_{\text {flux }-a m b} \cdot\left(T_{\text {amb }}-T_{\text {surf }}\right) \\
h_{\text {flux-amb }}=\lambda_{\text {air }} / h_{\text {ch }}
\end{gathered}
$$

\section{Heat flows in the system}

Ideally, power supplied to the heater is fully converted to thermal energy and remains confined in the central heater area. However, due to a variety of heat transfer mechanisms (conduction in solids and fluids, convection, radiation) the thermal energy is distributed in the membrane, the Si frame and the ambient gas. In this model we consider conduction in solids and surrounding gas, and we neglect natural convection and radiation (negligibly small for the studied temperature range). Forced convection due to the gas flow is considered in further sections. Possible paths for heat transfer from the micro hot plate and an equivalent thermal resistance circuit are shown

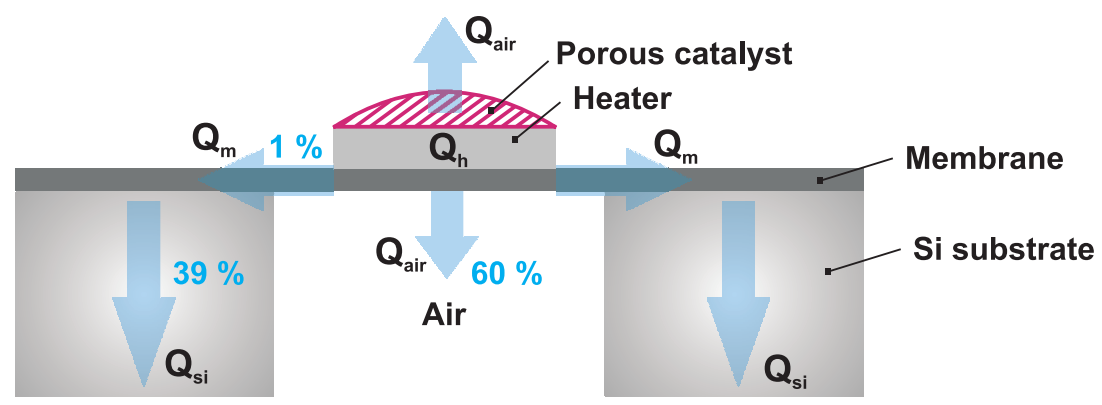

Figure 2.3: Overview of the heat flows in a catalytic sensor. Main heat sinks are Si substrate (ca. $39 \%)$ and surrounding air (flow and standing, ca. $60 \%$ ). The isolating membrane has a low thermal mass and itself takes less than $1 \%$ of the total supplied heat. However, it provides heat paths to Si and air due to the large surface-to-volume ratio.

in Figure 2.3 and Figure 2.4. The largest heat sink in the system is air (ca. $60 \%$ ) and silicon substrate (ca. $40 \%$ ). The overview of heat distribution calculated for different membrane materials is listed in Table 2.3. There is no drastic change in conduction loss mechanisms observed due to a comparable low thermal conductivity of the selected membrane materials. If the membrane would consist of silicon additionally to isolating layer as in microreactor system for catalytic partial oxidation [65], [66] then much higher heat fluxes would be allowed to flow to the silicon substrate and silicon-based membrane making heat flux towards the ambient negligible. In such a microreactor system, high supplied powers are necessary to compensate for the loss of heat through the membrane, as demonstrated the power raises almost up to $1 \mathrm{~W}$ [65]. Table 2.4 gives an overview of the calculated thermal resistances and thermal capacitances for the system with a $600 \mathrm{~nm}$ thick membrane considering an average $\left(T_{a v}\right)$ and maximum temperature $\left(T_{\max }\right)$ of the heater. This corresponds well 


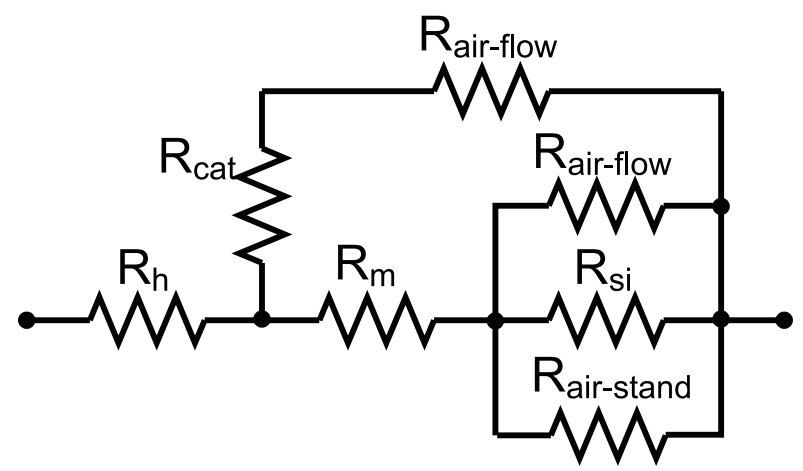

Figure 2.4: Equivalent circuit of thermal resistances: heat generated by the heater is transferred to the surrounding air (through catalyst layer and isolating membrane) and to Si substrate (through the membrane).

Table 2.3: Overview of the heat paths in a catalytic sensor system when $15 \mathrm{~V}$ potential (ca. $14 \mathrm{~mW}$ ) is applied: comparison is given for $\mathrm{SiRN}, \mathrm{SiO}_{2}, \mathrm{Si}_{3} \mathrm{~N}_{4}$ isolating membrane with thickness of 400 and $800 \mathrm{~nm}$.

\begin{tabular}{lccccc}
\hline & & \multicolumn{3}{c}{ \% of total supplied power } \\
\cline { 3 - 5 } & & \multicolumn{3}{c}{ Heat paths } & Useful heat \\
\cline { 3 - 5 } & $\mathrm{t}_{\text {memb }}(\mathrm{nm})$ & $\mathrm{Q}_{S i}$ & $\mathrm{Q}_{\text {memb }}$ & $\mathrm{Q}_{\text {air }}$ & $\mathrm{Q}_{h}$ \\
\hline \hline $\mathrm{SiRN}$ & 400 & 39.21 & 0.2729 & 60.49 & 0.0161 \\
& 800 & 39.2 & 0.5600 & 60.23 & 0.0149 \\
\hline $\mathrm{SiO}_{2}$ & 400 & 39.89 & 0.0167 & 59.81 & 0.0171 \\
& 800 & 39.00 & 0.0343 & 60.42 & 0.0159 \\
\hline $\mathrm{Si}_{3} \mathrm{~N}_{4}$ & 400 & 41.02 & 0.2751 & 58.69 & 0.0132 \\
& 800 & 42.72 & 0.5401 & 56.72 & 0.0121 \\
\hline
\end{tabular}

with the concept of high thermal resistance (or low thermal capacitance) desired in thermally efficient designs. Thermal resistances in the range of $27-59 \mathrm{~K} / \mathrm{mW}$ were determined, which is in a good agreement with the values measured for similar type of designs found in the literature [84]. The supplied power is expressed as sum of energies needed to rise the heater temperature, i.e. useful heat $\left(Q_{h}\right)$, and the heat transfer through membrane $\left(Q_{m e m b}\right)$, silicon substrate $\left(Q_{S i}\right)$ and air $\left(Q_{a i r}\right)$. By useful resistive heat $\left(Q_{h}\right)$ we mean heat needed to rise the temperature of the heater to desired level.

$$
Q_{\text {sup }}=I_{s r c} \cdot U=Q_{h}+Q_{m e m b}+Q_{s i}+Q_{\text {air }}
$$

The heat required to increase the temperature of the body is correlated to the thermal mass of the body $\left(\mathrm{C}_{p} \cdot \mathrm{m}\right)$ :

$$
Q=C_{p} \cdot m \cdot\left(T_{a v}-T_{a m b}\right),
$$

We extracted the average temperature $\left(T_{a v}\right)$ for each domain from the modeling results. The mass $(m)$ was calculated from the known volume $(V)$ and density $(D e)$ of the materials. The mass of the heater was expressed as a sum of the highly resistive part and the leads. We estimated that ca. $40 \%$ of total power supplied goes to silicon and the rest of the heat is distributed to the air. The heat transfer through the membrane $\left(Q_{\text {memb }}\right)$ and useful heat to warm up the heater $\left(Q_{h}\right)$ make nearly $1 \%$ of the total supplied power. The heat conducted through air $\left(Q_{\text {air }}\right)$ was calculated as the difference between the total supplied power $\left(Q_{\text {sup }}\right)$ and the sum of useful resistive heat and the heat transfer through the membrane and silicon $\left(Q_{m e m b}, Q_{S i}\right)$. 
Table 2.4: Overview of thermal resistances and thermal capacitances calculated at $T_{a v, h}$ along the heater and leads and at $T_{a v, \max }$ of the heater for $\mathrm{SiRN}, \mathrm{SiO}_{2}, \mathrm{Si}_{3} \mathrm{~N}_{4}$ isolating membrane with thickness of $600 \mathrm{~nm}$ and applied potential of $10 \mathrm{~V}(\mathrm{ca} .6 \mathrm{~mW})$.

\begin{tabular}{lcccccccc}
\hline Parameter & \multicolumn{2}{c}{$\mathrm{SiRN}$} & & \multicolumn{2}{c}{$\mathrm{SiO}_{2}$} & & \multicolumn{2}{c}{$\mathrm{Si}_{3} \mathrm{~N}_{4}$} \\
\cline { 2 - 3 } \cline { 8 - 9 } $\mathrm{T}_{a v, h} / \mathrm{T}_{a v, \max }(\mathrm{K})$ & 367.8 & 662.5 & & 371.54 & 727.6 & & 335.8 & 471.5 \\
\hline \hline $\mathrm{R}_{t h}(\mathrm{~K} / \mathrm{mW})$ & 11.34 & 59.31 & & 12.05 & 70.52 & & 5.96 & 27.44 \\
$\mathrm{C}_{t h}(\mathrm{nW} / \mathrm{K})$ & 88.2 & 16.8 & & 82.9 & 14.2 & & 167.8 & 36.4 \\
\hline
\end{tabular}

\subsubsection{D model of suspended heater in flow channel and chemical reaction}

A forced flow of reactive gas species over the heater and a catalyst pellet were included in the 2D model. We assume here that the heater design is optimized and results in an uniform temperature distribution at low power supply. The goal of this investigation is to correlate various flow, catalyst and reaction parameters relative to the dimensions of the suspended thermal actuator.

\section{Geometry, materials and boundary conditions}

The 2D geometry used in this study is presented in Figure 2.5. It consists of the suspended membrane structure with integrated heater dimensions identical to those discussed previously. All calculations are done with the assumption that physical processes in a third coordinate $\mathrm{z}$ are constant (extruded from 2D design). The system is placed in a channel with an inlet and an outlet for the transport of the reactive and non-reactive species. For non-reactive conditions the gas properties were taken as for air. A constant flow of gas was introduced into the channel. Thus, in addition to all heat loss mechanisms described previously, heat lost by forced convection is added. A porous catalyst pellet $\left(\mathrm{Pt} / \mathrm{Al}_{2} \mathrm{O}_{3}\right)$ with a radius varied from 1 to $50 \mu \mathrm{m}$ was placed on top of the heater. The properties of the catalyst are listed in Table 2.5. It is assumed that the solid structure has heat

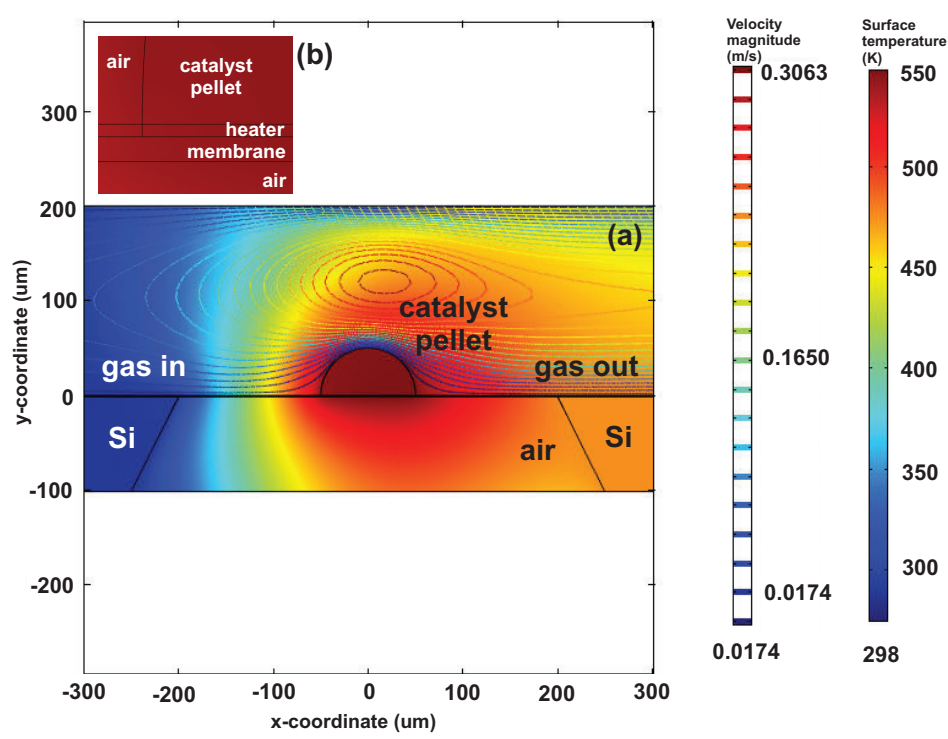

Figure 2.5: $2 D$ geometry used for modeling of the propane oxidation at constant flow conditions: temperature and flow velocity profiles are shown (a) with the overview of the sensor and catalyst arrangment $(b)$.

exchange with the environment. The top surface is subjected to forced convective heat exchange. Also, there is air below the membrane, which conducts heat. The normal heat flux to air is set to $130 \mathrm{~W} /\left(\mathrm{m}^{2} \cdot \mathrm{K}\right)$. This value is 
Table 2.5: Properties of the alumina catalyst.

\begin{tabular}{lcc}
\hline Parameter & Value & Units \\
\hline \hline Density, $D e_{A l}$ & 4000 & $\mathrm{~kg} / \mathrm{m}^{3}$ \\
Thermal conductivity, $\lambda$ & 30 & $\mathrm{~W} /(\mathrm{m} \cdot \mathrm{K})$ \\
Porosity, $\varepsilon_{g}$ & 50 & $\mathrm{vol} \%$ \\
Heat capacity, $C_{p}$ & $0.88 \cdot 10^{3}$ & $\mathrm{~J} /(\mathrm{kg} \cdot \mathrm{K})$ \\
Diffusion coefficient, $D$ & $1 \cdot 10^{-5}$ & $\mathrm{~m}^{2} / \mathrm{s}$ \\
Tortuosity, $\tau$ & 3 & - \\
Pt content, $c_{P t}$ & 10 & $\mathrm{wt} \%$ \\
\hline
\end{tabular}

calculated according to Equation 2.5 with the height of channel given as $200 \mu \mathrm{m}$. The inlet temperature of the gas is set to $293 \mathrm{~K}$ which equals to the ambient temperature surrounding the sensor.

\section{Transport of diluted species and energy balance}

There is fully developed laminar flow inside the channel by means of which reacting species are transported. Reynolds number in order of 10 was calculated. The fluid transport is described by the non-isothermal incompressible Navier-Stokes equations. At the inlet a velocity vector is specified normal to the boundary, the outlet boundary is specified by setting the pressure to 1 bar. The surfaces of the channel, heater and porous catalyst pellet are set to non-slip boundary conditions.

The concentration of the reacting gas is specified at the inlet. At the outlet the mass flow is governed by both diffusive and convective processes. It is assumed that no mass is transported across other boundaries. The energy balance equations consider heat transfer by means of convection $\left(Q_{c o n v}\right)$, diffusion $\left(Q_{d i f}\right)$ and conduction $\left(Q_{\text {cond }}\right)$. In gas the heat can be transferred by convection and conduction:

$$
D e_{\text {gas }} \cdot C_{p, g a s} \frac{\partial T}{\partial t}+\nabla \cdot(-\lambda \nabla T)+D e_{\text {gas }} \cdot C_{p, g a s} \cdot u_{\text {gas }} \cdot \nabla T=Q_{\text {gas }}
$$

Where $D e_{\text {gas }}$ denotes the density of gas, $C_{p, g a s}$ the heat capacity at constant pressure, $\lambda$ gas thermal conductivity, $u$ gas velocity and $Q_{\text {gas }}$ is a heat source. Within the catalyst conduction, diffusion and generation of the heat due to the reaction occur:

$$
D e_{c a t} \cdot C_{p, c a t} \frac{\partial T}{\partial t}+\nabla \cdot(-\lambda \nabla T)+R t \cdot \Delta H_{r}=Q_{c a t}
$$

The mass transfer is determined by the convection and diffusion equation including a reaction term $(R t)$ :

$$
\frac{\partial c}{\partial t}+\nabla \cdot\left(-D_{e f f} \cdot \nabla c+c \cdot u\right)=R t
$$

using the effective diffusion coefficient $\left(D_{e f f}\right)$, concentration $(c)$. The velocity $(u)$ of gas is set to zero as we assume that only diffusive processes occur within the catalyst.

Inside the catalyst pellet only the diffusive processes take place. We used an effective diffusion coefficient $\left(D_{e f f}\right)$ expressed by the density $(D e)$, porosity $\left(\varepsilon_{g}\right)$ and tortuosity $(\tau)$ :

$$
D_{e f f}=\frac{D e \cdot \varepsilon_{g}}{\tau}
$$

Finally, for the solids (micro hotplate materials) energy balance equation includes only conduction:

$$
D e_{s o l} \cdot C_{p, s o l} \frac{\partial T}{\partial t}+\nabla \cdot(-\lambda \nabla T)=Q_{s o l}
$$



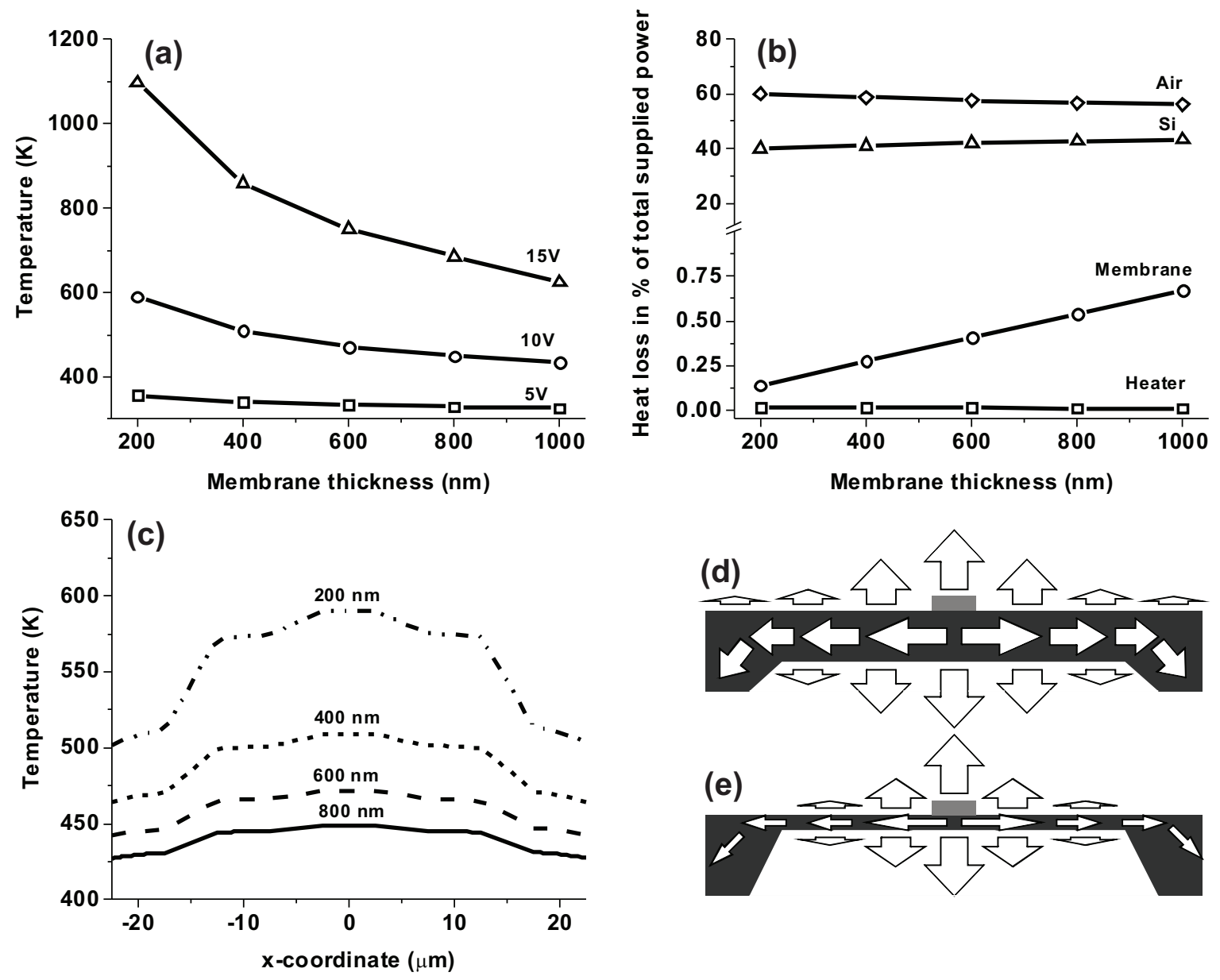

Figure 2.6: Influence of the $\mathrm{Si}_{3} \mathrm{~N}_{4}$ membrane thickness on the maximum temperature of the heater at potential applied of 5, 15, $15 \mathrm{~V}$, corresponding to ca. 2, 6, $14 \mathrm{~mW}($ a), heat paths in \% of total supplied power (b), surface temperature distribution (c) at $10 \mathrm{~V}(6 \mathrm{~mW})$ and scheme of heat flows in thick (d) and thin (e) membrane-based sensor (arrow thickness represents the size of cross sectional area and arrow length represents the heat flux).

\subsection{Results and discussion}

\subsubsection{Design parameters}

\section{Thickness of membrane}

The influence of membrane thickness was investigated for the case of a $\mathrm{Si}_{3} \mathrm{~N}_{4}$ membrane.

Shown in Figure 2.6a is the maximum temperature of the heater at the membrane thickness varied between 200 and $1000 \mathrm{~nm}$ and applied potentials of 5, 10 and $15 \mathrm{~V}$ which corresponds to ca. 2, 6, $12 \mathrm{~mW}$ supplied power, respectively. The thinner the membrane, the smaller path of conduction to the substrate, which represents the largest heat sink in the system besides the surrounding air as can be seen from Figure 2.6b. Therefore, with thinner membranes better localization of the heat at the high-ohmic central part can be achieved. Shown in Figure 2.6c are surface temperature profiles across the heater area at the membrane thickness varied between 200 and $1000 \mathrm{~nm}$ and applied potential of $10 \mathrm{~V}$. The general trend is that with the increase of membrane thickness less temperature gradient was found in the heated area. The lowest gradient is observed for $800 \mathrm{~nm}$ thick membrane. Thus, increase in thickness, leading to increase in thermal mass, causes decrease of maximum achievable temperature, but better temperature uniformity. The heat flows within the micro hot plate comprised of the thin and thick isolating membrane are illustrated in Figures 2.6d and 2.6e. 

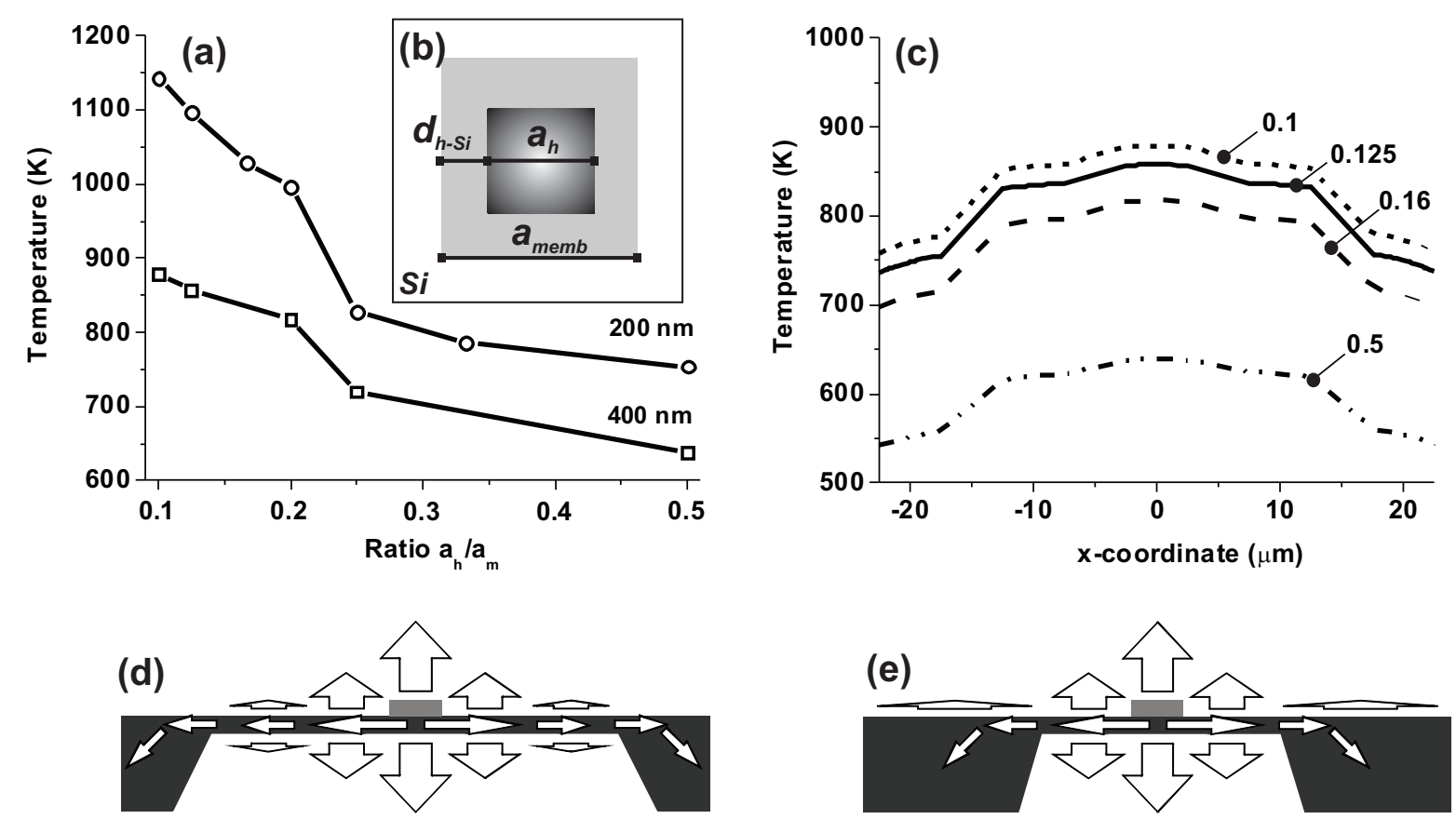

Figure 2.7: Influence of the $\mathrm{a}_{h} / \mathrm{a}_{\text {memb }}$ ratio illustrated on the top view (a) on the maximum temperature of the heater for membrane thickness of 200 and $400 \mathrm{~nm}(b)$, surface temperature distribution for membrane thickness of $400 \mathrm{~nm}(\mathrm{c})$ at potential applied of $15 \mathrm{~V}$ (corresponds to supplied power of ca. $14 \mathrm{~mW}$ ) and scheme of heat flows in membrane-based sensor with low $\mathrm{a}_{h} / \mathrm{a}_{\text {memb }}(d)$ and high $\mathrm{a}_{h} / \mathrm{a}_{\text {memb }}(e)$ ratios (arrow thickness represents the size of cross sectional area and arrow length represents the heat flux).

\section{Ratio of heater and membrane}

The influence of the $\mathrm{a}_{h} / \mathrm{a}_{m}$ ratio on the maximum temperature on the membrane and surface temperature distribution is illustrated in Figure 2.7a. Heat flux in the membrane can be expressed in terms of thermal conductance $\left(\lambda_{\text {memb }}\right)$, distance between heater and silicon $\left(d_{h-s i}\right)$ and temperature difference between the heater $\left(T_{h}\right)$ and substrate $\left(T_{S i}\right)$ :

$$
q=\frac{\lambda_{m e m b}}{d_{h-s i}} \cdot\left(T_{h}-T_{S i}\right)
$$

The larger the $d_{h-s i}$ the less heat is lost to the substrate.

Shown in Figure 2-7b is the dependence of the maximum heater temperature on $a_{h} / a_{m}$ ratio. The smaller ratios, in general, result in higher total heat losses (losses to surrounding air do not change but losses through substrate become larger). This tendency is not linear: for $a_{h} / a_{m}$ ranging from 0.2 to 0.5 the heat losses increased. This is more pronounced for a $200 \mathrm{~nm}$ thick membrane where the thermal gradient is higher. The optimum for the cavity size can be selected in the ratios less than 0.25 . At ratios below 0.25 the main heat loss mechanism is by conduction through the air, thus, more heat will be supplied to catalyst and reacting gas.

Shown in Figure 2-7c are the temperature profiles for various ratios of $a_{h} / a_{m}$ for $\mathrm{Si}_{3} \mathrm{~N}_{4}$ membrane with a thickness of $400 \mathrm{~nm}$. At high ratios $a_{h} / a_{m}$ no improvement in the temperature uniformity is observed. Only the absolute temperature is decreased due to the proximity of the Si substrate to the heating source and higher thermal flux towards the substrate. The heat flows within the micro hot plate with small and large cavity are illustrated in Figures 2.7d and 2.7e. 


\section{Leads dimensions}

At all other optimized parameters the design of the contact leads is crucial. The balance has to be found between lower electrical conductance, to localize heat, and low thermal conductance, to reduce the heat flux from the area of the leads to air and Si substrate. These two effects are counterbalancing each other: better heat confinement is achieved with larger leads, but, on the other hand, increased heat losses via thermal conductive process are introduced.

The influence of the width of force leads $\left(w_{\text {flead }}\right)$ on heat transfer within the sensor was investigated at the assumption of stationary conditions (zero convective flux) and all other dimensions of the heater as specified earlier in Table 2.1. In Figure 2.8 is presented the maximum temperature of the heater and total supplied power as a function of width of force leads. When increasing the width from $15 \mu \mathrm{m}$ to $50 \mu \mathrm{m}$ gain in temperature of ca. $120 \mathrm{~K}$ can be achieved with power difference of only $1 \mathrm{~mW}$. Above $100 \mu \mathrm{m}$ the increase in width does not lead to further increase in temperature. The optimum width of force leads is in the range 40-100 $\mu \mathrm{m}$ for the case of negligible convective losses. If the convective losses would be introduced the total supplied power would increase drastically with increased area of the leads. Therefore, we expect that optimum width of the force leads resulting in localization of the heat at the center of the membrane and acceptable conductive heat losses is ca. $50 \mu \mathrm{m}$ (ratio of 10/1 of $w_{\text {flead }} / w_{h}$ ).
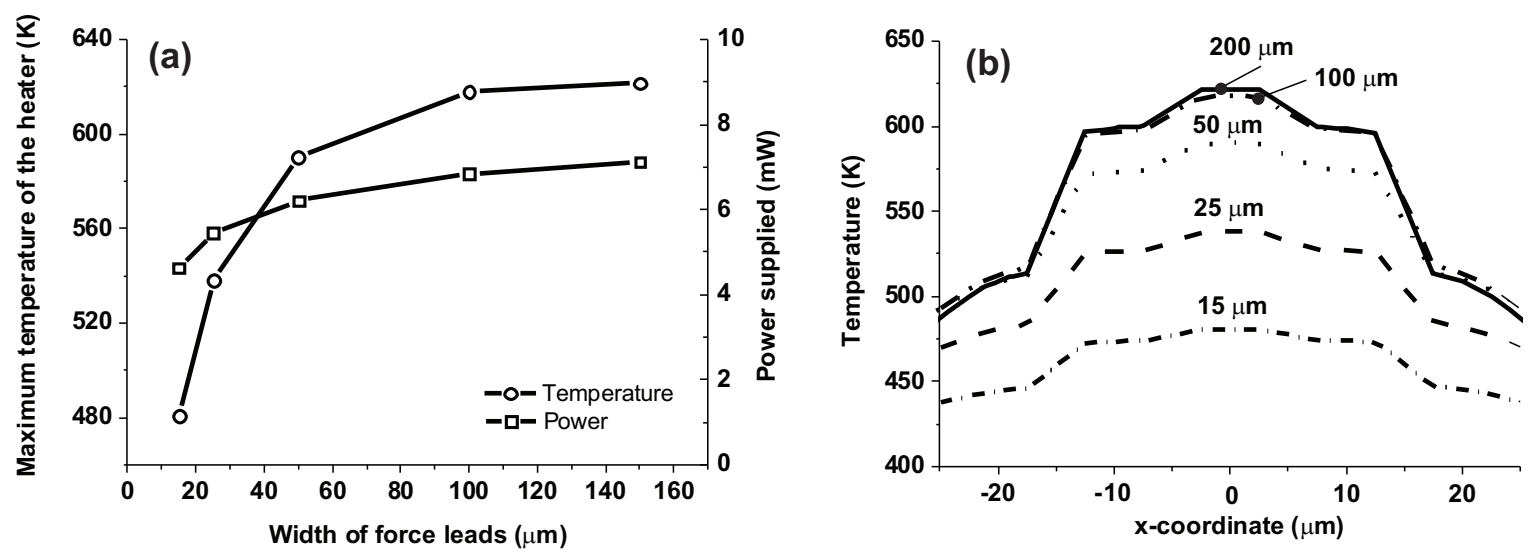

Figure 2.8: Influence of the force leads width on the maximum temperature of heater and total supplied power (a) and temperature distribution across active area of the sensor as a function of source lead width(b).

\subsubsection{Materials of micro hotplate}

\section{Membrane}

Influence of the thermal conductive properties of the isolating membrane on temperature on the membrane and surface temperature distribution are summarized in Figure 2.9. Selection of a substrate with lower thermal conductive properties, such as $\mathrm{SiO}_{2}$ and $\mathrm{SiRN}$ increases the maximum temperature of the heater surface by about 150-250 K with all other conditions equal, and therefore, improves the desired local heating effect at lower total supplied power. Based on modeling results a higher temperature can be achieved in micro hotplates based on $\mathrm{SiO}_{2}$ and $\mathrm{SiRN}$ membranes compared to $\mathrm{Si}_{3} \mathrm{~N}_{4}$. However, from the practical point of view, SiRN low stress thin films is a preferred choice due to their mechanical strength and coefficient of thermal expansion close to $\mathrm{Si}$ (see Table 2.2).

For comparison, decreasing membrane thickness from 1000 to $200 \mathrm{~nm}$ increases temperature by less then 100 $\mathrm{K}$. Thus, the thermal conductivity of the membrane material has a higher impact on thermal losses than variation in thickness because the thermal mass anyway remains small. This influence becomes even more pronounced at higher temperatures (see Appendix A). 

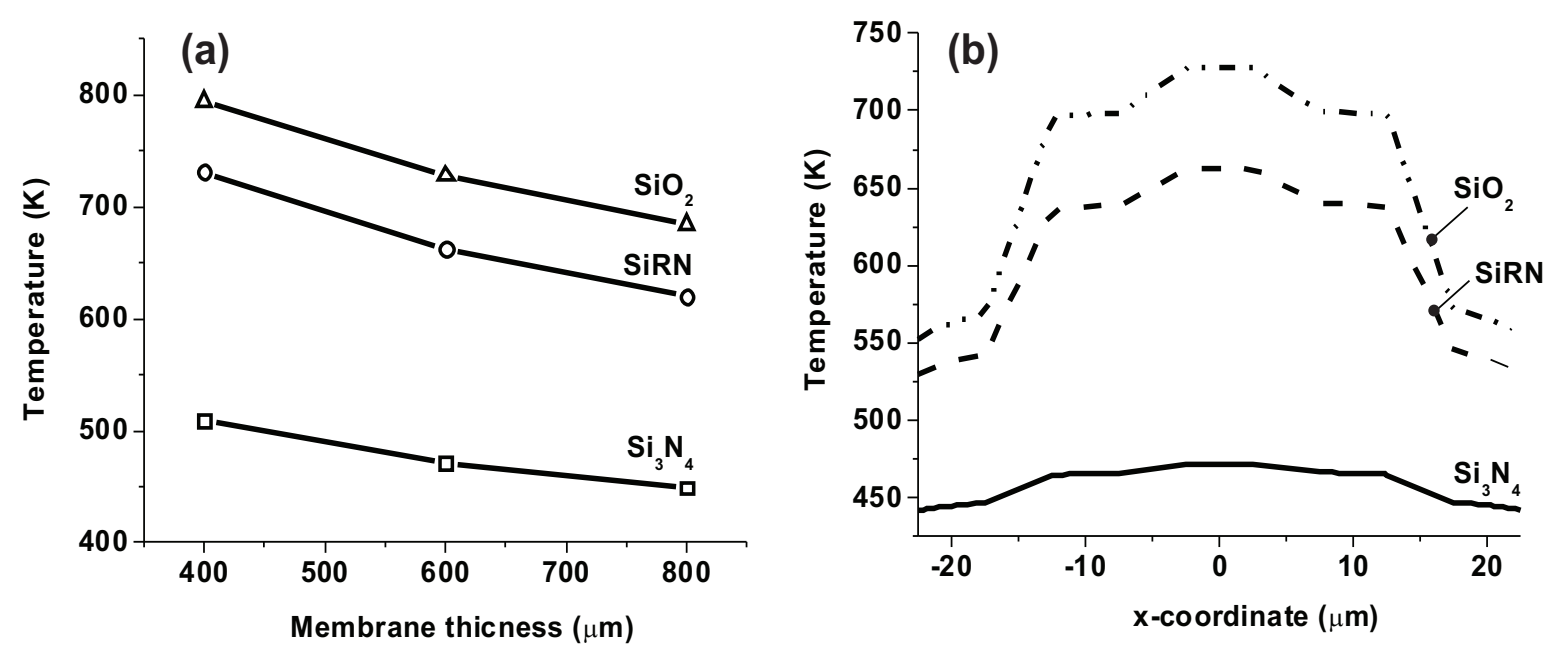

Figure 2.9: Influence of the membrane material on the maximum temperature of the heater (a) and surface temperature distribution for membrane thickness of $600 \mathrm{~nm}(\mathrm{~b})$ at potential applied of $10 \mathrm{~V}$ (corresponding to supplied powers in the range 5-7 $\mathrm{mW}$ ).

In Figure $2.9 \mathrm{~b}$ temperature profiles are shown for a $600 \mathrm{~nm}$ thick membrane for various membrane materials. As can be seen, although $\mathrm{Si}_{3} \mathrm{~N}_{4}$ has less favorable thermo-physical properties in terms of achieving local heating effect at decreased power consumption, it had the best temperature distribution due to its higher conductive values.

\section{Substrate}

The second largest heat sink in the system after air is the Si substrate. In order to reduce these losses a substrate with lower thermal resistance can be used, such as fused silica. The thermal conductance of fused silica is significantly smaller, but the heat capacity is of the same order as Si. To reduce the thermal mass the membrane is commonly released from the backside by silicon etching. We compared to cases:

- Quartz substrate;

- Quartz substrate with the thin $\mathrm{SiO}_{2}$ membrane.

If quartz is used as a substrate, and additionally, the cavity is etched below the heater, the total power consumption can be significantly reduced compared to a Si substrate. Shown in Figure 2.10 is the dependence of the temperature on supplied power for a Si-based micro hotplate with a $\mathrm{Si}_{3} \mathrm{~N}_{4}$ membrane (a) and a fused silica based hot plate with the released membrane (b). As can be seen the thermal efficiency of the latter one is much higher. In Figure 2.11 influence of the thickness of the released membrane in the fused silica based hot plate is shown. Selection of membrane material with low thermal conductivity as well as thinner membranes lead to decrease in power consumption. However, there are practical limitations imposed by mechanical stability: thermally induced stress in thinner membranes and compatibility of thermal expansion coefficients of all materials (see Table 2.2). Also, the implementation of a thin, low thermally conductive membranes introduces strong temperature gradients - hot spots - increasing as the thickness of the membrane becomes smaller, see Figure 2.12. For the case of a non-suspended heater the power consumption exceeds desirable limits, i.e. below 15-20 $\mathrm{mW}$, as shown in Figure 11a. However, the temperature gradient is the lowest in this case and estimated to be $0.35 \mathrm{~K} / \mu \mathrm{m}$ (Figure $2.12 \mathrm{~b}$ ). If high temperature uniformity is the prioritized requirement then the membrane should be kept thick (500-1000 nm) or not released at all. If power needs to be decreased a membrane can be introduced, but thicknesses larger than $800 \mathrm{~nm}$ are feasible in terms of the temperature uniformity. 

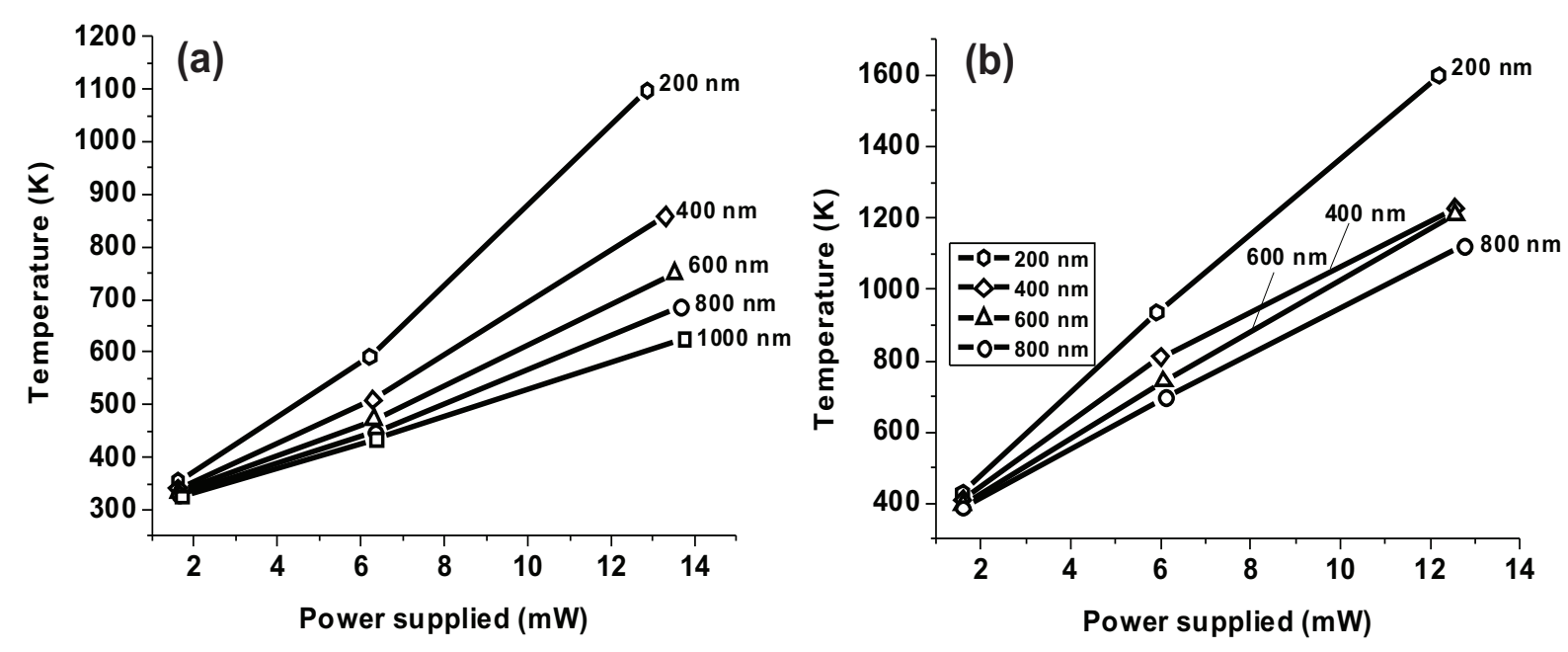

Figure 2.10: Dependence of the heater temperature suspended on the $\mathrm{Si}_{3} \mathrm{~N}_{4}$ membrane of 200-1000 nm thick in Si-based micro hotplate (a) and when integrated on the fused silica substrate with the released membrane with the thickness varied between 200-800 $\mathrm{nm}(b)$.
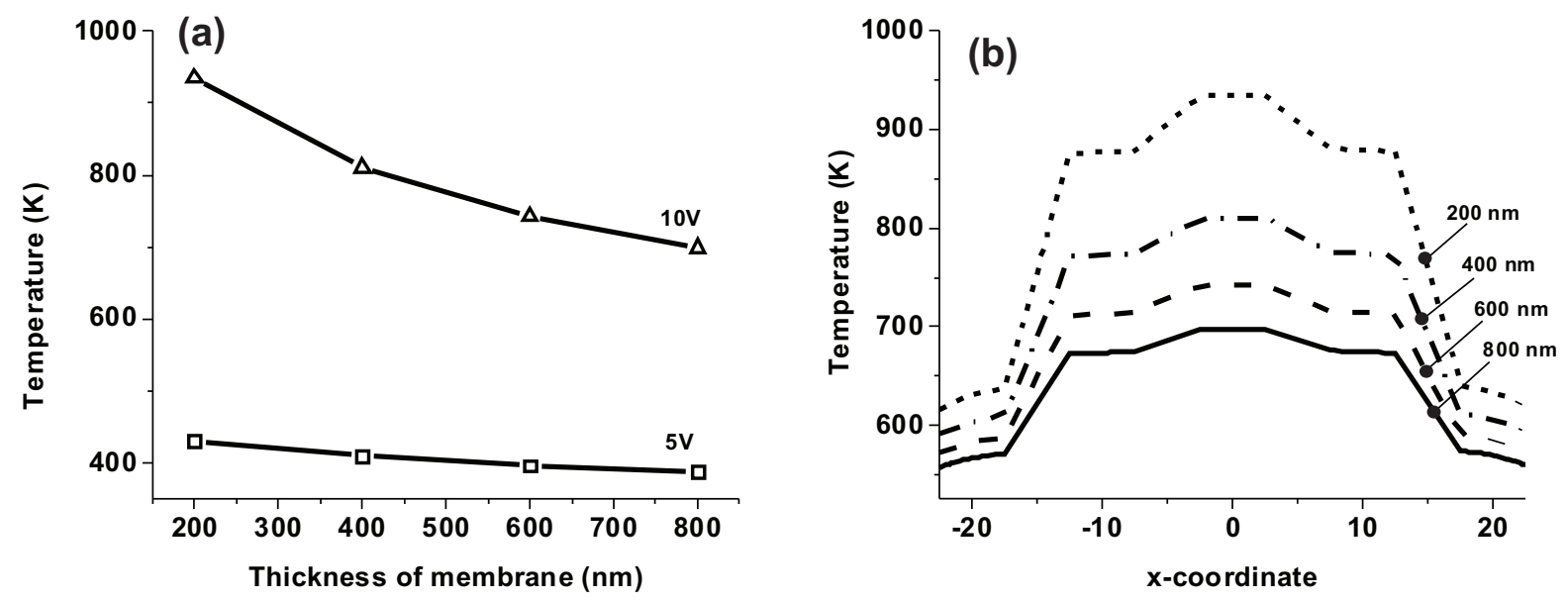

Figure 2.11: Influence of the thickness of the released $\mathrm{SiO}_{2}$ membrane on quartz substrate on the maximum temperature of the heater $(a)$ and surface temperature distribution for various thicknesses $(b)$ at potential applied of $10 \mathrm{~V}$ (corresponds to supplied powers in the range 6-7 $\mathrm{mW}$.

Optimization of the temperature profile can be done by incorporation of heat distributors on the back side of the membrane [85]. In our system, a catalyst layer integrated on top of the heater with high thermally conductive properties (metal thin films) also serves as a heat distributor.

\section{Thermal response}

Transient thermal modeling is outside the scope of this study, however it is highly important. The thermal time constant is greatly affected by the thermal isolating properties of the membrane and the identification of dominant time constants in the system is important. When the system needs to operate dynamically to monitor reactions, the response time of the total system needs to be proportional to the response of the sensing area. Thermal response is also dependent on thermal diffusivities of respective heater and membrane materials. The 

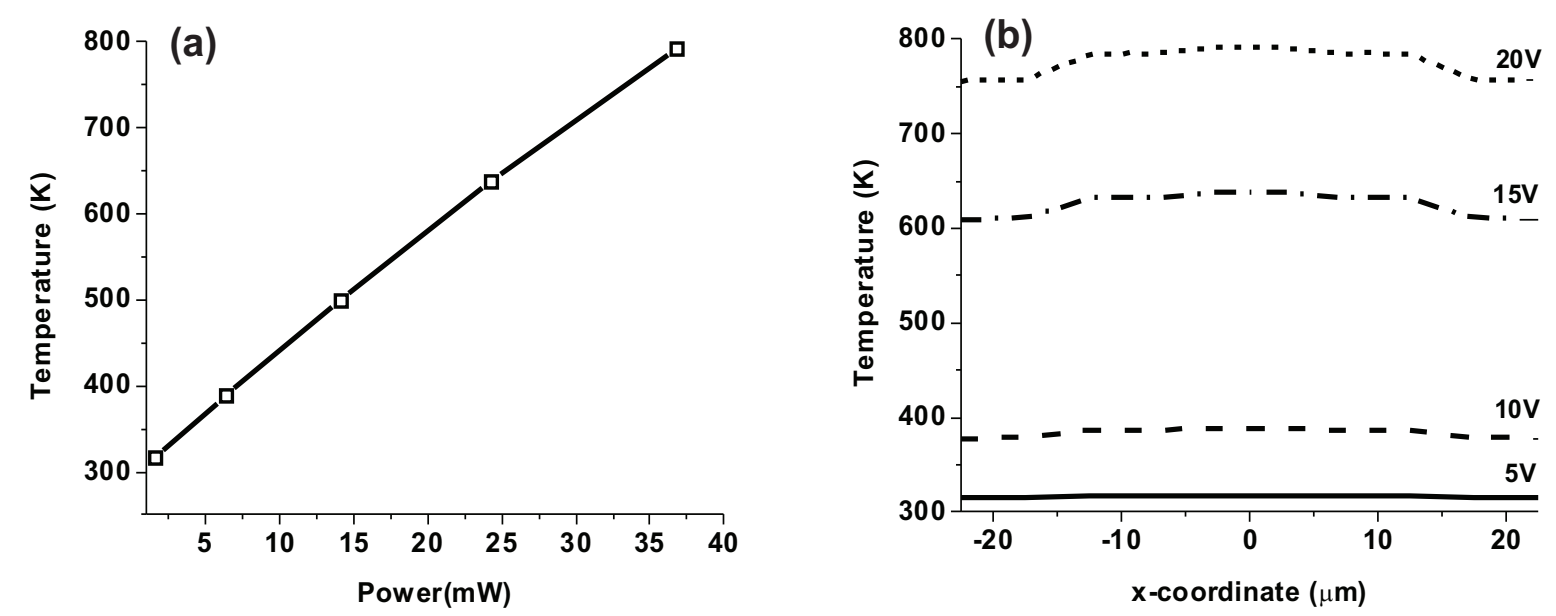

Figure 2.12: For the fused silica substrate: dependence of the maximum temperature of the heater on the supplied power (a) surface temperature distribution (b) at potential applied of 5, 10, 15, $20 \mathrm{~V}$ (corresponds to $1,7,14,25 \mathrm{~mW})$.

heat diffuses faster in Si than in oxides or nitrides. The presence of a substrate introduces an additional time constant which might be higher than that of the heater itself as the thermal mass of membrane and substrate together is much higher. From the point of view of fast response, the substrate could be eliminated completely by suspending the heater on its leads. This reduces the heating up time. If the same element is intended to work as a heater as well, and rapid cooling times are needed it would be impossible to cool down the system quickly due to the limited conduction path through the suspended leads. Additionally, in our case full integration into the channel structure is required, so the presence of a membrane is necessary to define the dimensions of the channel and the flow. The properties of gas have a minor influence on the transient behavior of the device because the heat diffuses in gases many times slower and the heat capacity is small. Therefore, the optimization with respect to the response time is based on the choice of the membrane material and thickness mainly.

\subsubsection{Flow and chemical reaction}

In Figure 2.5 the temperature and gas velocity profile (a) and a close up in the heater-catalyst interface (b) are shown. In Figure 2.13 the concentration profile in the channel is shown. We assume here that our catalystreactive gas combination has certain kinetic parameters, which are taken from L. van de Beld et al. [86], and the initial parameters for the reaction summarized in Table 2.6. The reaction in the system is actuated by means of a resistive heat source $\left(P_{\text {res }}\right)$. Besides the resistive heat, the heat due to the reaction $\left(P_{\text {reac }}\right)$ is present and expressed as:

$$
P_{\text {reac }}=-Q_{o x} \cdot k \cdot e^{\left(-E_{a} /\left(R_{i d} \cdot T\right)\right)} \cdot c \cdot R_{i d} \cdot T \cdot\left(1-\varepsilon_{g}\right) / \varepsilon_{g} \cdot D e_{A l} \cdot c_{P t}
$$

The reaction rate is expressed as:

$$
r_{\text {reac }}=-k \cdot e^{\left(-E_{a} /\left(R_{i d} \cdot T\right)\right)} \cdot c \cdot R_{i d} \cdot T \cdot\left(1-\varepsilon_{g}\right) / \varepsilon_{g} \cdot D e_{A l} \cdot c_{P t},
$$

where $Q_{o x}$ - enthalpy of combustion, $k$ - pre-exponential factor, $E_{a}$ - activation energy, $\varepsilon_{g}$ - porosity, $D e_{A l}$ - density listed in Table 2.5 and Table 2.6. Several factors influence the sensitivity to evolved heat: the temperature under the catalyst (or supplied power), the concentration of the gas, the flow rate (cooling effect), the thermal conductivity of the gas, the concentration of Pt active catalyst and the dimensions of both catalyst and heater. We discuss separately each of these factors. Shown in Figure 2.14a is the heat effect due to the reaction as a function of the concentration of propane in air and supplied power is shown. With the increase of power, the catalyst surface becomes hotter and the reaction rate rises exponentially in accordance with Arrhenius equation 


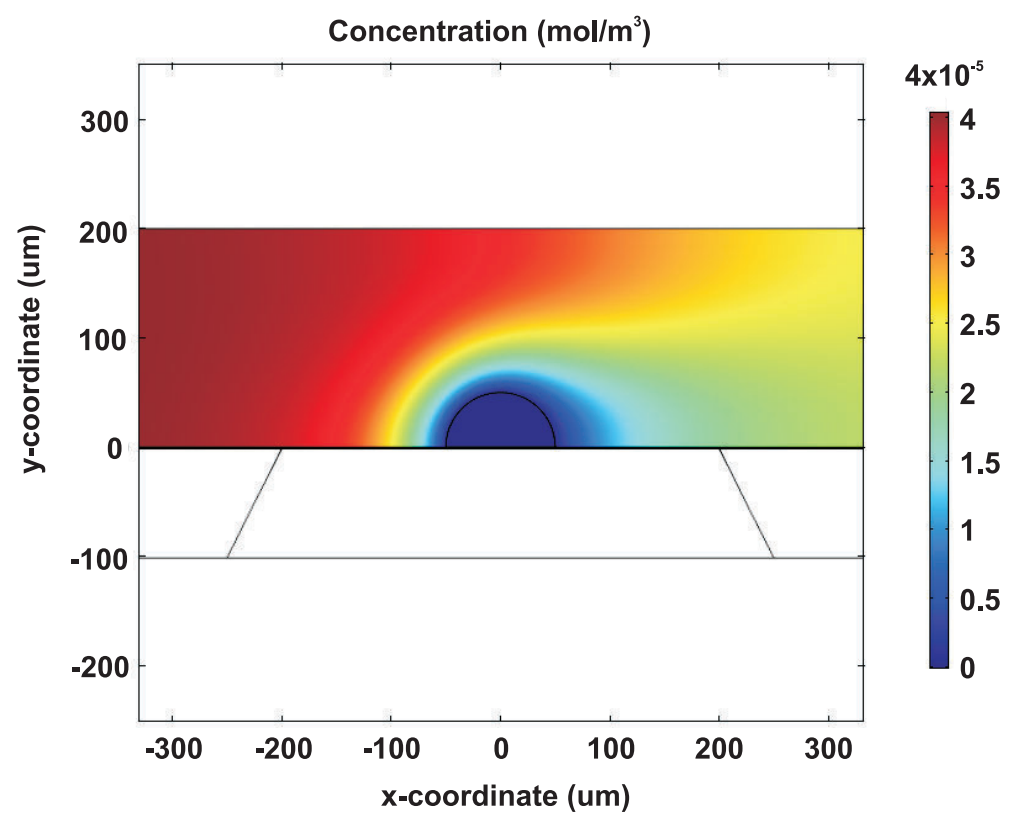

Figure 2.13: Concentration profile inside the channel.

Table 2.6: Parameters of the propane oxidation in air implemented in the model.

\begin{tabular}{lcc}
\hline Parameter & Value & Units \\
\hline \hline Enthalpy of combustion, $\Delta_{r} \mathrm{H}$ & $-2043 \cdot 10^{3}$ & $\mathrm{~J} / \mathrm{mol}$ \\
Activation energy, $\mathrm{E}_{a}$ & $92.9 \cdot 10^{3}$ & $\mathrm{~J} / \mathrm{mol}$ \\
Pre-exponential factor, $\mathrm{k}$ & 14 & $\mathrm{~mol} /(\mathrm{kg} \cdot \mathrm{s} \cdot \mathrm{Pa})$ \\
Inflow velocity, $\mathrm{u}_{\text {in }}$ & $0.1 / \mathrm{variable}$ & $\mathrm{m} / \mathrm{s}$ \\
Outlet pressure, $\mathrm{p}_{\text {out }}$ & $1 \cdot 10^{5}$ & $\mathrm{~Pa}$ \\
Inlet temperature, $\mathrm{T}_{\text {in }}$ & 298.15 & $\mathrm{~K}$ \\
Initial concentration of propane in air, $\mathrm{c}_{i n}$ & variable & $\mathrm{ppm}$ \\
\hline
\end{tabular}

(Equation 14-15). Therefore, the highest temperature differences are achieved at the interface of the heater and the catalyst. The larger the amount of reacting gas, the more heat is produced. If low concentrations are to be detected, higher reaction temperatures (higher power supplied) should be applied, to compensate for lower conversions, because at low gas concentrations slow kinetics (low reaction rate) occur. Shown in Figure 2.14b is the heat effect as a function of the gas flow rate. When a flow is present in the channel the difference in temperature, compared to stationary conditions, is due to the forced convective heat transfer by the gas. When the flow is increased, the measured temperature difference decreases not only due to the cooling effect and losses, but also due to the decreased time of contact between gas and catalyst (residence time). Therefore, operation of catalytic sensors at very small flow rates and/or stationary conditions is preferred. In stationary (non-flow) conditions, reactants are delivered by means of diffusion, thus the response of such detection system would be limited by time for gas to diffuse to the catalyst. On the other hand, when steady-state flow conditions are used, the catalyst is at all time supplied with fresh reactants and the response time is faster.

Shown in Figure 2.14c is the heat effect as a function of the Pt content in alumina. The larger the amount of catalyst, assuming the same catalyst dispersion, the more the reaction proceeds and the higher the temperature difference can be obtained (see the term for Pt concentration in Equation 14, 15). From the Figure 2.14c it can be seen that at concentrations above $20 \mathrm{wt} \%$ the limit is approached. This is due to a non-optimum use of $\mathrm{Pt}$, i.e. only on the outer shell of the pellet the reaction will take place. Practically, at higher catalyst loading the dispersion decreases. In fact, in industrial catalysts the amount of Pt usually does not exceed 1-5 wt \% to get 

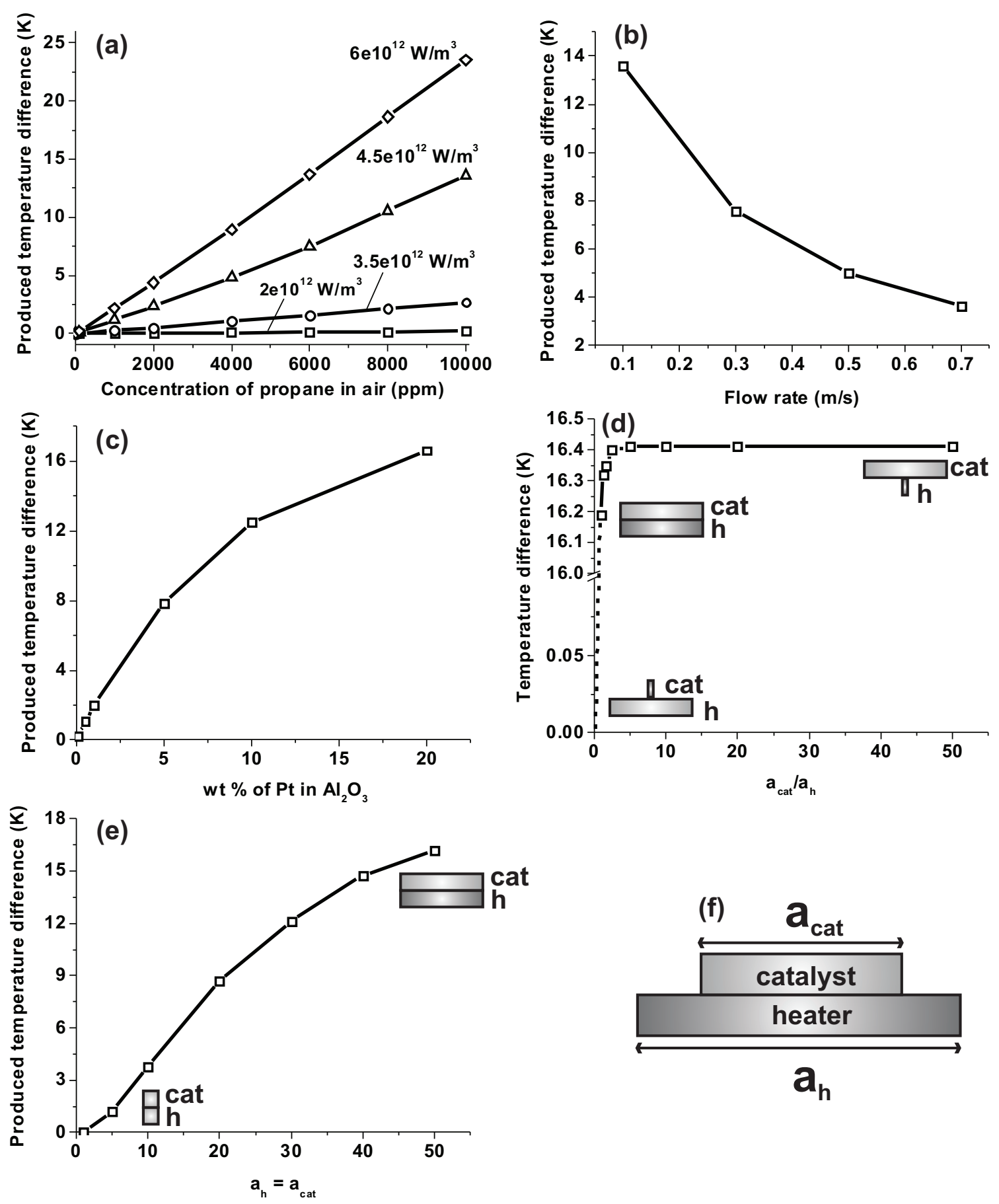

Figure 2.14: Dependence of the heat effect (maximum temperature difference) at the catalyst-heater interface on concentration of propane in air fed into the channel at various supplied power ranging from $(2-6) \times 10^{12}$ $\mathrm{W} / \mathrm{m}^{3}$ at flow rate of $0.1 \mathrm{~m} / \mathrm{s}($ a), on flow rate at concentration of propane $10000 \mathrm{ppm}$ and $10 \mathrm{wt} \%$ Pt content (b) and on Pt content at concentration $10000 \mathrm{ppm}$ and flow rate $0.1 \mathrm{~m} / \mathrm{s}$. The resistive heat source for (b) and (c) was $5 \times 10^{12} \mathrm{~W} / \mathrm{m}^{3}$; the dependence of temperature difference on ratio of length of catalyst to length heater (d) and on length of heater (when equal to length of catalyst) (e) at $5 \times 10^{12} \mathrm{~W} / \mathrm{m}^{3}$ power, 10000 ppm propane in air, $20 \% \mathrm{Pt}_{\mathrm{A}} \mathrm{l}_{2} \mathrm{O}_{3}$ and $0.1 \mathrm{~m} / \mathrm{s} ;(f)$ schematic representation of dimensions which were varied in modeling. 
Table 2.7: Dependence of heat loss mechanisms in the system on thickness and material of isolating membrane and $a_{h} / a_{\text {memb }}$ ratio.

\begin{tabular}{lccc}
\hline Heat flux & $\mathrm{t}_{\text {memb }} \uparrow$ & $\mathrm{a}_{h} / \mathrm{a}_{\text {memb }} \uparrow$ & $\lambda_{\text {memb }} \uparrow$ \\
\hline \hline $\mathrm{Q}_{h}$ & $\downarrow$ & $\downarrow$ & $\downarrow$ \\
$\mathrm{Q}_{\text {memb }}$ & $\uparrow$ & $\uparrow$ & $\uparrow$ \\
$\mathrm{Q}_{S i}$ & $\uparrow$ & $\uparrow$ & $\uparrow$ \\
$\mathrm{Q}_{\text {air }}$ & $\downarrow$ & $\downarrow$ & $\uparrow$ \\
$\mathrm{Q}_{\text {tot }}$ & $\uparrow$ & $\uparrow$ & $\uparrow$ \\
T uniformity & $\uparrow$ & $\uparrow$ & $\uparrow$ \\
\hline
\end{tabular}

higher dispersion.

Shown in Figure 2.14d the heat effect as a function of the dimensions of the heater $\left(a_{h}\right)$. The ratio of length of catalyst ( $a_{\text {cat }}$ kept constant $50 \mu \mathrm{m}$ ) to various lengths of the heater $\left(a_{h}\right)$ was used as a variable. The supplied power $\left(Q_{\text {sup }}\right)$ was kept constant and the resistive heat source $\left(P_{r e s}\right)$ was recalculated according to dimensions of the heater. This recalculation is the result of the $2 \mathrm{D}$ model.

Three cases are presented in Figure 2.14d:

1. $a_{c a t} / a_{h}<1$;

2. $a_{\text {cat }} / a_{h}=1$;

3. $a_{c a t} / a_{h}>1$.

For the first case, when $a_{c a t}$ is much smaller than $a_{h}$, the heater surface will be party exposed to the ambient introducing additional paths for conductive and convective heat transfer to the surroundings.

When $a_{c a t} / a_{h}$ is nearly one, the upward heat flux from the heater is only possible through the catalyst layer, which is more efficient way of heating.

As can be seen in Figure 2.14d, a further increase in $a_{c a t} / a_{h}$ ratio does not introduce substantial temperature differences (ca. $0.02 \mathrm{~K}$ ). Even if the $a_{c a t} / a_{h}$ ratio of 50 would be possible to realize in practice, a heater of minimized dimension might burn out before the catalyst reaches the required temperature level. To conclude, reducing size of heater while keeping the catalyst dimensions the same does not improve the performance.

When catalyst and heater length are assumed identical (see Figure 2.14e) downscaling their dimensions results in a decrease in produced temperature difference. This is due to the fact that a smaller amount of catalyst volume produces less heat. At the same time smaller dimensions lead to a reduction of power consumption. Therefore, in this case, downscaling is feasible. However, the sensitivity of the sensor must be sufficiently high to detect induced temperature differences.

In general, in order to keep the produced temperature difference high (for the detection) the ratio of the dimensions of catalyst that of the heater must be at least 2.5. At the ratio of 2.5 a maximum produced temperature difference can be reached at a minimum power conditions.

\subsection{Summary and conclusions}

Optimization of micro hotplate design parameters is application specific. Based on the modeling results the general conclusions are drawn and summarized in Table 2.7:

1. The implementation of thinner membranes leads to an overall decrease in power consumption of the micro hotplate but at the same time reduces uniformity of the thermal distribution across the active heater area. 
2. The implementation of the membranes comprising of low thermally conductive materials, such as $\mathrm{SiO}_{2}$ and $\mathrm{SiRN}$ reduces conductive losses to the Si substrate, and, thus increases the thermal efficiency. At the same time the temperature uniformity decreases, and formation of hot spots in the heater area is possible.

3. For the range of selected materials and conditions, it was found that the material of the membrane influences heat loss mechanisms more than the thickness of the membrane at all other constant parameters.

4. The ratio $\mathrm{a}_{h} / \mathrm{a}_{m}$ taken below 0.25 reduces losses to the Si substrate and does not lead to a strong decrease in the uniformity of the surface temperature profiles.

5. The main heat transfer paths are to Si substrate (ca. $40 \%$ ) and to air (ca. $60 \%$ ). The amount of heat lost by heating the membrane is less than $1 \%$ of the total supplied power. However, the membrane introduces a heat path to the Si substrate and air, thus, the thickness and, especially material of the membrane need to be selected based on the application specific power consumption requirements.

6. Conduction through air is dominating loss mechanism due to the high surface area of the membrane exposed to air. Air conduction can be reduced by operating the device in a medium with a lower thermal conductivity.

7. Heat effect of reaction is directly proportional to concentration of a target gas (propane) in air and actuating temperature. If a catalyst is not active enough, higher temperatures can be applied to compensate for it.

8. Non-flow operating conditions is preferred operational mode if a higher thermal sensitivity is required. This is due to the fact that the heat losses are less compared to constant flow operation. In contrary, in flow mode a faster response is achieved.

9. Higher temperature of the catalyst and thus, higher reaction rate and evolved amount of heat due to oxidation, is achieved when dimensions of the heater and catalyst are comparable. Further downscaling of the heater while keeping the catalyst dimensions constant is not feasible.

10. When catalyst and heater dimensions are assumed identical downscaling results in a decrease in produced temperature difference. This is due to the fact that a smaller amount of catalyst volume produces less heat. At the same time smaller dimensions lead to a reduction of power consumption. Therefore, in this case, downscaling is feasible. However, the sensitivity of the sensor must be sufficiently high to detect induced temperature differences.

11. In order to keep the produced temperature difference high (for the detection) the ratio of the dimensions of catalyst that of the heater must be at least 2.5. At the ratio of 2.5 a maximum produced temperature difference can be reached at a minimum power conditions. 


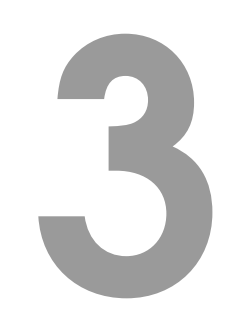

\section{Measurement of reaction heats using a polysilicon-based microcalorimetric sensor *}

In this work we present a low-cost, low-power, small sample volume microcalorimetric sensor for measurement of reaction heats. The polysilicon-based microcalorimetric sensor combines several advantages: (i) complementary metal oxide semiconductor technology (CMOS) for future integration; (ii) elements of silicon micromachining (MEMS) to control thermal performance; (iii) heterogeneous catalysts for selective detection and analysis of individual gas compounds; and (iv) microfluidics for optimized control over the reaction conditions. A comprehensive study on the electrical properties of polysilicon thin films as a potential material for temperature monitoring of highly exothermic reactions is presented. Resistive measurements were performed up to $800^{\circ} \mathrm{C}$ and a temperature coefficient of resistance (TCR) of $3.24 \times 10^{-4} \rho \mathrm{C}$ was derived in the quasi-linear resistive range between 300 and $500{ }^{\circ} \mathrm{C}$. The polysilicon thin film temperature sensors show a good stability. Due to its excellent compatibility with silicon technology and chemical inertness, doped polysilicon thin films can be successfully applied in catalytic microreactors and sensor microsystems at high operating temperatures. We demonstrate the performance of polysilicon sensors by the detection of reaction heats for a model reaction - the catalytic oxidation of propane in air at concentrations in the range of $0.01-0.8 \mathrm{vol} \%$ which is below the low explosion level (LEL) of propane of $2.1 \%$ [37]. The sensor exhibits immediate and reversible response upon exposure to propane in air. By choosing a selective catalyst the specificity of the sensor can be tuned to different gases.

${ }^{*}$ Published as: E.Vereshchagina, R.A.M.Wolters, J.G.E.Gardeniers, Measurement of reaction heats using a polysilicon-based microcalorimetric sensor, Sens. Actuators A 169 308-316. 


\subsection{Introduction}

\subsubsection{Microcalorimetric sensors}

Microcalorimetric sensors belong to the group of thermal chemical sensors and detect the heat of a reaction, released or consumed. This change in enthalpy associated with a (bio-) chemical reaction introduces a temperature change, which can be converted into an electrical signal and measured. The rate of a chemical reaction depends on temperature and concentration of target compounds. Therefore, the determination of the heat evolved provides a means of measuring the gas concentration [37], [36]. One of the ways to initiate a reaction is to use a heterogeneously catalyzed system. In a typical measurement, the device is brought in contact with a solid catalyst that lowers the operating temperature of the sensor and provides a degree of control over the reaction kinetics. The basic elements of a catalytic sensor are a temperature sensor, a heater and a catalyst. The performance of a catalytic sensor is determined by each of these three elements [87].

There is a demand for microcalorimetric devices in various fields due to the simplicity and the selectivity of a microcalorimetric measurement. The detection and analysis of chemical components is vital for various industrial, commercial, research and domestic applications. Commonly, the gas reactants and by-products exhibit similar physical properties, but need to be monitored individually. Additionally, a fast response of the sensor in combination with the possibility to design a highly specific sensor are factors of main concern during the development of a gas sensing and analysis system. This counts especially for the detection of hazardous gases for environmental and automotive exhaust control [88], [10], where response time and selectivity play a crucial role.

Microcalorimetric sensing also has become an industrial method for measuring the flows of both gases and liquids [89]. On the application of microcalorimetry in microfluidics an informative review by W. Lee and coworkers was published [35]. Furthermore, microcalorimetric detection is also widely applied in bioanalytical chemistry [90]. An overview of various designs of microcalorimeters for (bio-) chemical analysis was given by A.W. van Herwaarden [34]. Finally, microcalorimetric sensors have been successfully applied in the process control of integrated microreactors for gas-phase catalytic reactions [91], [92], [93]. An important issue in this application is the possibility of maintaining similar reaction conditions in various channels during scale-up and automation.

This work focuses on microcalorimetry as a detection method. It is the only method providing a direct indication of the reaction kinetics by absolute measurement of reaction heats. The ultimate goal of this study is to develop a microfluidic system in which microcalorimetry is applied for catalytic detection of gases, kinetics studies, but also for catalyst screening, study of catalyst deactivation processes and measurement of reaction, adsorption or desorption heats. We use a solid catalyst immobilized on a transducer surface. The catalytic oxidation of various hydrocarbons (methane, ethane, propane, etc.) occurring at elevated temperatures was selected as a target reaction. Furthermore, high temperature handling within $100-450{ }^{\circ} \mathrm{C}$ is required for ona-chip activation and regeneration of catalysts. For these reasons it is important that resistive characteristics of functional material do not degradate under the influence of temperature.

As a proof of concept we study the oxidation of propane in air for concentrations of propane within 0.01 0.8 vol \% using a CMOS compatible, low-power micro hot plate based sensor. The oxidation reaction is exothermic and the released reaction heat is directly proportional to the gas concentration. This is used for calibration. The actuating temperature of the catalytic oxidation (typically up to $550{ }^{\circ} \mathrm{C}$ [94], [95]) and the measured total reaction heats are parameters which are specific for individual gas compounds. In this way an effective differentiation between compounds of common nature may be achieved by using one single chip.

\subsubsection{Feasibility of polysilicon integration}

In this study we implement thin films of doped polycrystalline silicon (polysilicon) as resistive heaters and temperature sensors. Polysilicon is a widely studied material and its implementation in microsensor technology was investigated extensively [96]. However, its properties still find new and exiting applications in CMOS/MEMS design, e.g. thermoelectric power generators [97], temperature sensors on a chip for study of polymerase 
chain reaction (PCR) [98] or micromachined neural probes [99]. This demand for polysilicon as a material for high temperature sensing and microreactor applications is justified by its excellent compatibility with standard CMOS and MEMS technologies, its stability and chemical inertness at high temperatures.

Platinum (Pt) is a preferred material for high-temperature sensing applications [100]. Polysilicon, unlike commonly used Pt thin films, does not catalyze any (undesired) reactions. In microsystems comprising catalysts and applied for the synthesis or analysis of fine chemicals it is of special importance that all elements except the catalyst exhibit zero catalytic activity. Although it is possible to circumvent this problem for Pt resistors, by encapsulating it between catalytically inactive passivation layers [101], reliable use of Pt requires a stable adhesion layer. Most of the issues related to a Pt reliability are due to a degradation of this adhesion layer at temperatures above ca. $550^{\circ} \mathrm{C}$. Polysilicon thin films do not require such adhesion layers.

Processing of polysilicon has some advantages compared to metal thin films. It enables high temperature operations and, can, therefore, be easily passivated with high quality thermal oxide or nitride to prolong the life-time of a resistor. Ehmann and co-workers investigated the properties of polysilicon in harsh temperature environments and demonstrated its reliable operation for temperatures up to $1200 \mathrm{~K}$ [102].

Futhermore, polysilicon can be used not only as a material for heating and sensing, but also as a thermopile, a piezoresistive component or as a mechanical support in bulk micromachining. This is especially important for construction of smart sensor systems comprising devices of various functionalities.

In addition, as a part of the gobal trend, silicon-based sensors and microreactors need to be technologically merged with MEMS/NEMS. In this view, polysilicon-based microdevices easily enable high temperature postCMOS processing steps, such as temperature-assisted anodic bonding.

\subsection{Experimental}

\subsubsection{Design overview}

The prototype calorimetric sensor consisted of heavily boron doped polysilicon heaters, temperature sensors and a patterned catalyst integrated on a freely suspended Silicon-Rich Nitride (SiRN) membrane. The active area of the sensor was selected as $300 \times 300$ or $500 \times 500 \mu \mathrm{m}$. The membrane thickness was selected as $200 \mathrm{~nm}$ and $400 \mathrm{~nm}$. The top view of the active area of the sensor is shown in Figure 3.1. Our approach was to deposit the catalytic interface on top of a transducer platform, consisting of a thin low-stress membrane containing resistive structures for heating and temperature sensing. The catalyst was deposited locally in such a way that it can be heated efficiently using thin-film polysilicon heaters. Subsequently, the start and path-way of catalytic reactions can be controlled with integrated polysilicon temperature sensors. These were located up- and downstream relative to the gas flow and used for continuous temperature monitoring. The thickness of the SiRN membrane was selected to minimize the heat losses to the silicon. Additionally, the SiRN membrane ensured faster response times compared to designs completely isolated by air and simplified the integration of the calorimetric sensor into the glass microfluidic channel. The function of channel is to carry out the oxidation reaction in a controlled manner (laminar flow, Re number below 10). The generated heat was concentrated locally on the active surface of the sensor and supplied mainly to the catalyst. The gas flown over the sensor surface and below the membrane acted as an efficient thermal isolator. Compared to the heat loss by radiation, convection and conduction through the air, thermal conduction through the contact leads and the membrane are the main paths for heat losses. Therefore, an optimization of the thermal and electrical resistances of the suspended-membrane heater and the minimization of heat loss through the electrical interconnections was our main concern during the design stage. Literature on hot plate design optimization can be found elsewhere [103].

\subsubsection{Fabrication of the microcalorimetric sensor}

\section{Fabrication of the transducer platform}

The main steps of the fabrication are shown schematically in Figure 3.2. A low-doped p-type double-side polished $<100>$ silicon wafer with a thickness of $525 \mu \mathrm{m}$ and a diameter of $100 \mathrm{~mm}$ was used. $200 \mathrm{~nm}$ of 


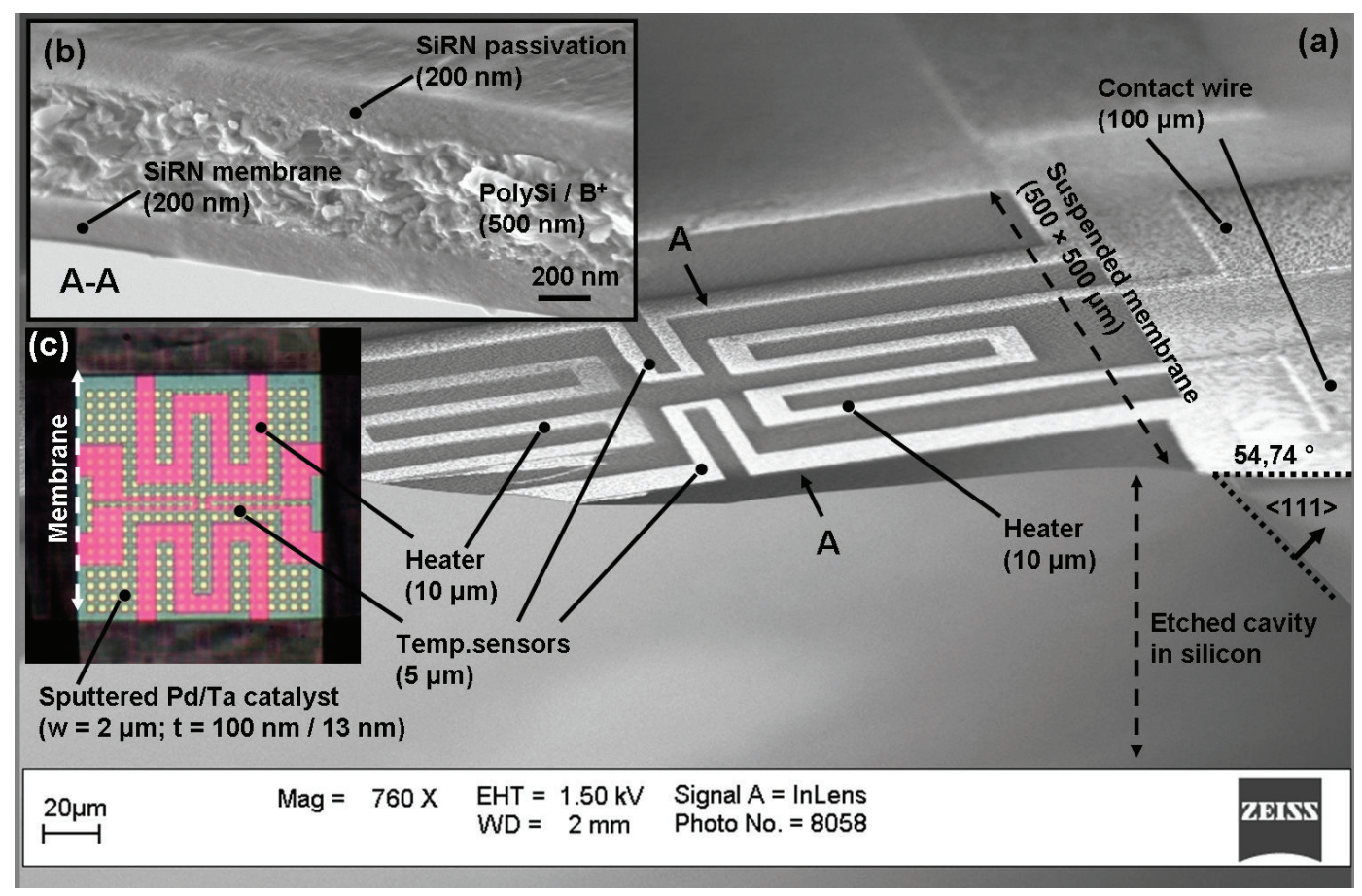

Figure 3.1: HRSEM image of the sensor device after silicon etching (a), cross-sectional view on the SiRN/PolySi/SiRN membrane (b), microscopic image made from the back side of the released membrane showing the arrangement of heaters and temperature sensors (c).

Low Pressure Chemical Vapor Deposition (LPCVD) SiRN at $850{ }^{\circ} \mathrm{C}$ was deposited using $\mathrm{SiCl}_{2} \mathrm{H}_{2}$ and $\mathrm{NH}_{3}$ gases. The deposition of the SiRN layer was followed by growth of $500 \mathrm{~nm}$ of LPCVD polycrystalline silicon at $620{ }^{\circ} \mathrm{C}$ from $\mathrm{SiH}_{4}$. To lower the resistance of the polysilicon, $\mathrm{B}^{+}$was implanted with a dose of $5 \times 10^{15} \mathrm{at} / \mathrm{cm}^{2}$ and an activation energy of $80 \mathrm{keV}$. This resulted in a sheet resistance $\left(\mathrm{R}_{\text {sheet }}\right)$ of polysilicon thin films in the range of $190-220 \Omega / \mathrm{sq}$ and a $\mathrm{B}^{+}$impurity concentration of about $10^{20} \mathrm{~cm}^{-3}$. In order to remove lattice defects and activate the dopants the ion implantation step was followed by conventional thermal annealing in nitrogen at $800^{\circ} \mathrm{C}$ for $30 \mathrm{~min}$. Patterning of polysilicon into resistive structures was achieved by standard photolithography and Reactive Ion Etching (RIE) in $\mathrm{SF}_{6}, \mathrm{CHF}_{3}$ and $\mathrm{O}_{2}$ plasma. The fabricated heaters and temperature sensors were passivated with a $200 \mathrm{~nm} \mathrm{SiRN} \mathrm{thin} \mathrm{film} \mathrm{to} \mathrm{isolate} \mathrm{them} \mathrm{electrically} \mathrm{and} \mathrm{chemically} \mathrm{from} \mathrm{the} \mathrm{catalyst}$ layer. Openings through the SiRN to the polysilicon were etched using a RIE process with a $\mathrm{CHF}_{3}-\mathrm{O}_{2}$ plasma. Subsequently, $500 \mathrm{~nm}$ of aluminum was sputtered and patterned using lithography and the standard aluminum wet etchant (Merck 115435.200). Metallization was followed by annealing in $\mathrm{N}_{2}$ at $450{ }^{\circ} \mathrm{C}$ for $20 \mathrm{~min}$ to form a stable ohmic contact to the polysilicon heating and temperature sensing structures. Thermal insulation was realized by bulk micromachining of silicon, i.e. anisotropic wet etching in $25 \%$ potassium hydroxide solution $(\mathrm{KOH})$ at $75{ }^{\circ} \mathrm{C}$ with $\mathrm{SiRN}$ as an etch-stop layer. During single-side silicon etching, the front of the wafer containing the functional structures was protected by a stainless steel holder.

\section{Immobilization of the catalytic interface}

A thin film of palladium (Pd) was used as model catalyst for the investigation of propane oxidation. Patterning of the catalyst was done prior to the membrane release. $100 \mathrm{~nm}$ of Pd on top of a $15 \mathrm{~nm}$ thick tantalum (Ta) adhesion layer were DC magnetron sputtered and patterned via a lift-off process (30 min ultrasonification in acetone bath), resulting in a square pattern. Ta was selected as an adhesion layer for Pd due to its stable performance as an adhesion promoter at high temperatures [104]. 

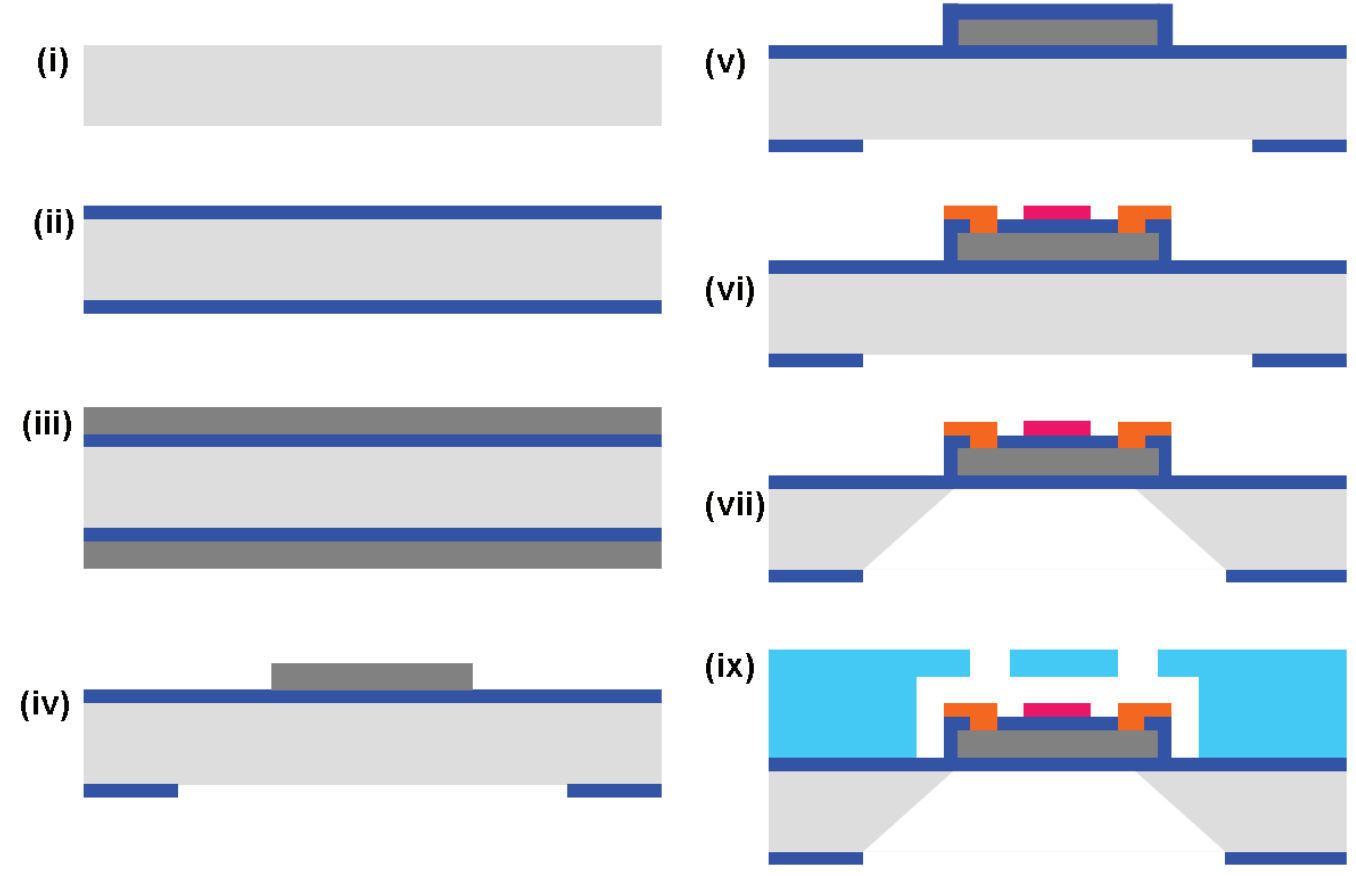

Figure 3.2: Fabrication process of the sensor device: (i) wafer selection, (ii) deposition of the $200 \mathrm{~nm} L P C V D$ SiRN, (iii) deposition of the $500 \mathrm{~nm}$ LPCVD PolySi, (iv) definition of resistive structures by the RIE plasma etching, (v) deposition of the $100 \mathrm{~nm}$ LPCVD SiRN for passivation, (vi) fabricatiion of the contact pads and patterning of catalytic material, (vii) etching of Si and SiRN membrane release, (ix) bonding with glass wafer, dicing and further assembling.

\section{Integration into the microfluidic channel}

Microfluidic channels were formed in borofloat glass wafers with a thickness of $1100 \mu \mathrm{m}$ and a diameter of $100 \mathrm{~mm}$ supplied by Schott AG (Grünenplan, Germany). Channels of 200 and $700 \mu \mathrm{m}$ height were etched in hydrofluoric (HF) water acid solutions of $25 \%$ and $50 \%$, respectively, through a gold (Au) - on - chromium (Cr) mask.

Thin films of $\mathrm{Au}(60 \mathrm{~nm})$ on $\mathrm{Cr}(10 \mathrm{~nm})$ were deposited by DC magnetron sputtering and patterned using standard Au (KI: Merck 105043, $\mathrm{I}_{2}$ : Merck 144761, KI:I $\mathrm{I}_{2}: \mathrm{DI}$ water $\left.=(4: 1: 40)\right)$ and $\mathrm{Cr}($ Merck 111547.2500) etchants. Holes for fluidic connections and electrical probing were formed using powder blasting. Channels were manually aligned with regard to the active area of the sensor and anodically bonded at $400{ }^{\circ} \mathrm{C}$ and a potential stress up to $1800 \mathrm{~V}$.

In the final stage, the silicon-glass wafer stack was diced to samples. Individual sensor devices were glued and wire-bonded on top of a Printed Circuit Board (PCB) (Figure 3.3).

\subsubsection{Electrical characterization}

\section{Polysilicon thin films}

TCR and stability of the polysilicon thin films were characterized at temperatures up to $800^{\circ} \mathrm{C}$. For the electrical characterization at high temperatures we used double-side polished fused silica wafers of Q1 quality with a diameter of $100 \mathrm{~mm}$ and a thickness of $525 \mu \mathrm{m}$ supplied by Schott AG. $500 \mathrm{~nm}$ of LPCVD polycrystalline silicon was deposited on the fused silica and subjected to ion implantation as discussed in section 2.1. Fused silica substrates were selected because this material allows temperature rampings up to $800{ }^{\circ} \mathrm{C}$ during the resistive measurements on the thin films. Additionally, we performed similar analyses on p-type $<100>$ single crystal silicon wafer passivated with $200 \mathrm{~nm}$ LPCVD low-stress SiRN prior to deposition of polysilicon. 


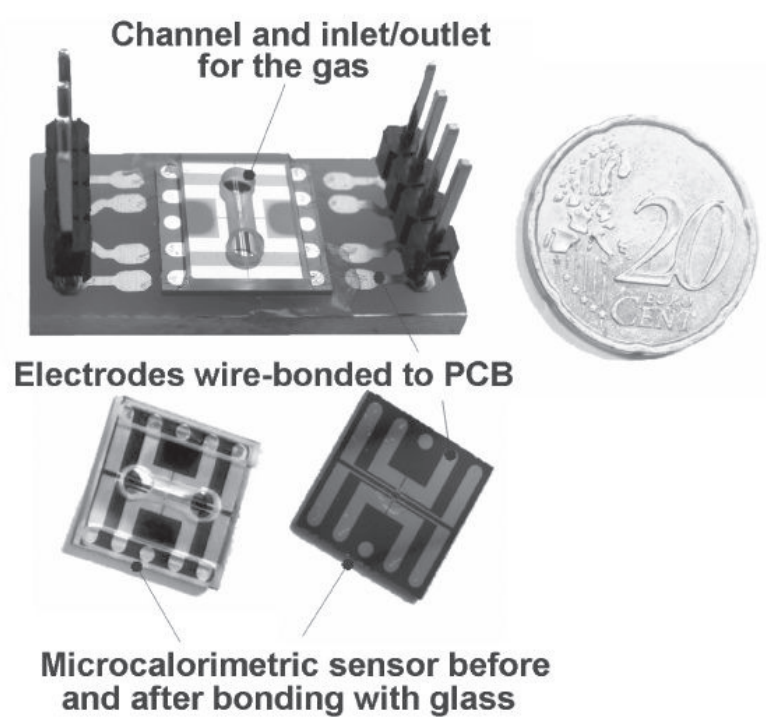

Figure 3.3: The sensor device before and after the glass-bonding and mounted on the PCB. The channel dimensions are: height $200 \mu \mathrm{m}$, width $600 \mu \mathrm{m}$, length $4500 \mu \mathrm{m}$.

These tests were, however, only valid in the lower temperature range due to the conductive properties of SiRN at temperatures above $250{ }^{\circ} \mathrm{C}$. This effect was investigated in detail by Tiggelaar et al. [105].

We used a measurement setup equipped with four probes at fixed positions $(16 \times 16 \mathrm{~mm})$ evacuated during the operation, a heating element and an aluminum nitride substrate holder permitting temperature ramps up to $800{ }^{\circ} \mathrm{C}$. The substrate was connected to the holder using a silver thermal paste on the holder to ensure good thermal contact. The temperature was regulated via a thermo-couple positioned directly below the sample. All resistive measurements were performed at $100 \mathrm{mV}$. Measured values of resistance $\left(R_{\text {meas }}\right)$ were recalculated to $R_{s}$ and resistivity $(\rho)$ :

$$
R_{S}=R_{\text {meas }} \times C_{F},
$$

where $C_{F}$ is a correction factor ( 6 for the given geometry).

$$
\rho=R_{S} \times t
$$

where $t$ is the thickness of the resistor.

The dependence of $R_{\text {meas }}$ on the temperature can be generally expressed as:

$$
R_{\text {meas }}=R_{0}\left(1+\alpha \times\left(T-T_{0}\right)\right)
$$

The $\alpha$ was extracted from the linear fitting parameters $\mathrm{R}_{l i n 0}$ and $\beta$ of the $R_{\text {meas }}(T)$ curve:

$$
\begin{gathered}
R_{\text {lin }}=R_{\text {lin } 0}+\beta \times T \\
\alpha=\beta / R_{\text {lin } 0} .
\end{gathered}
$$

\section{Polysilicon resistors}

During the fabrication of the sensor, electrical measurements on Greek Cross resistive test structures [106] were performed for process control. IV measurements on final devices were carried out using a HP4156B (HewlettPackard, Palo Alto, CA, USA) Precision Semiconductor Parameter Analyser in combination with a Cascade Microtech Probe station equiped with a heating chuck permiting temperatures up to $175^{\circ} \mathrm{C}$.

Calibration of polysilion resistors was carried out using the calibrated heating chuck. Series of IV's were 
collected in the range from room temperature to $175{ }^{\circ} \mathrm{C}$. The resistive values corresponding to the temperatures above $175^{\circ} \mathrm{C}$ were measured by placing the chip to the temperature programmed oven. Temperature of the oven was regulated externally by a thermocouple. From the collected data the TCR was extracted (see Equations 3.33.5). The temperature of the membrane was recalculated using the $\mathrm{R}$ (time) data from the integrated resistive temperature sensor and assuming the constant TCR. The current sourced to resistors used for temperature sensing is kept at $100 \mu \mathrm{A}$.

\section{Experimental study of propane oxidation}

The reaction of propane in air on a Pd catalyst was investigated. The sensor signal is registered under various electrical and fluidic operational conditions. The heater and the sensor were operated in constant power mode using an Agilent 34970A (Agilent, Santa Clara, CA, USA) data acquisition unit and an Agilent U2722A source measure unit. All electrical parameters of heater and temperature sensors were monitored and controlled with Labview, National Instruments Netherlands BV (Woerden, The Netherlands) program customized in house. It allowed an independent and flexible setup and readout of all the involved control parameters in either constant power or constant temperature modes.

The gases were fed independently and at a constant pressure to the sensor using Brooks Instrument BV (Ede, The Netherlands) precision mass flow controllers. The total flow rate in the system was kept at $3 \mathrm{sccm} / \mathrm{min}$. Instrumental air (purity 4.8) and nitrogen (purity 5.0) were fed continuously. Two 4-way micro electric actuators were used to switch between a nitrogen line and a propane/nitrogen mixture. The concentration of propane in the propane/nitrogen mixture was $1 \mathrm{vol} \%$. Nitrogen was used as a balance gas for the propane mixture in order to keep the flow rate constant and minimize effects of thermal conductivity. By diluting the standard mixture of propane/nitrogen (1/99 vol \%) with an additional nitrogen the pulses of propane at various concentrations and flow rates were injected. The concentration of propane in nitrogen was varied between $0.01-0.8 \mathrm{vol} \%$. The flows for all gases were calculated based on a stoichiometric equation for the total oxidation of propane, shown in Equation 3.6 [75].

$$
\mathrm{C}_{3} \mathrm{H}_{8}+5 \mathrm{O}_{2} \Longrightarrow 3 \mathrm{CO}_{2}+4 \mathrm{H}_{2} \mathrm{O}\left(\Delta_{r} \mathrm{H}^{0}=-2220.1 \mathrm{~kJ} / \mathrm{mol}\right)
$$

The sensor chip was placed in a polyoxymethylene plastic housing. It was designed to allow an easy replacement of the sensor. The gas-tight fluidic connectors and capilaries were installed in the sensor housing.

Time and duration of the gas pulses were controlled. At first a baseline resistance corresponding to a nitrogenair flow was measured. Then we introduced the pulses of propane/nitrogen into the sensor channel. Propane reacted with oxygen from air on the catalytic surface of the chip. This resulted in a generation of the heat and a subsequent increase in the sensor resistance. Based on the resistive measurements the heat release of the reaction was identified and compared with the conversion data from a Varian BV (Middelburg, The Netherlands) CP-4900 micro gas chromatograph (Micro-GC) equipped with molsieve and propak Q columns. Chromatographic data allowed us to correlate the heat release with the reaction rate.

\subsection{Results and discussion}

\subsubsection{Polysilicon as a material for microcalorimetric sensing}

We studied the resistive properties of the polysilicon thin films and validated the feasibility of polysilicon in the temperature-assisted microreactor and sensing systems.

\section{Resistive properties of polysilicon}

$\mathrm{B}^{+}$doping is a preferred option to alter the resistance of heating and sensing structures in our application. According to Mandurah et al., n-type doping substances such as arsenic and phosphorus may lead to segregation effects at the grain boundaries [107]. The parameters of the implantation process, a dose of $5 \times 10^{15}$ ions $\cdot \mathrm{cm}^{-2}$ 
and an energy of $80 \mathrm{keV}$, were selected based on modelling results to obtain uniform dopants distribution in polysilicon. The heavily-doped thin polysilicon films indicated metal-like behaviour with $R_{S}$ within $173-220$ $\Omega / \mathrm{sq}$ and $\rho$ of about $9.5-11 \mathrm{~m} \Omega \cdot \mathrm{cm}$ for $500 \mathrm{~nm}$ thick films. A higher sensitivity and a faster response can be obtained in intrinsic and moderately doped thin films. It is, however, more practical to utilize extrinsic polysilicon due to a better stability of its electrical characteristics. The obtained temperature dependence of the
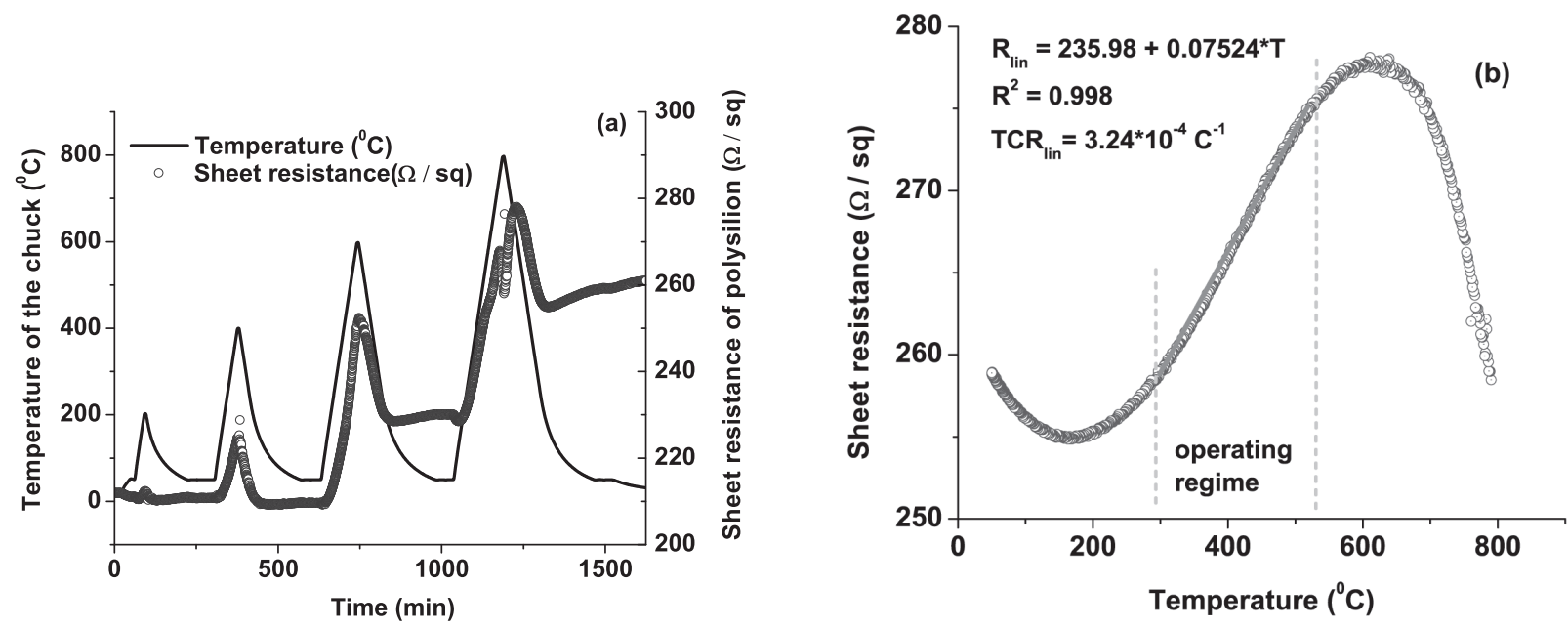

Figure 3.4: The sheet resistance of thin polysilicon films deposited on fused silica in time under temperature cycling conditions between 50 and 200,400, 600 and $800^{\circ} \mathrm{C}(\mathrm{a})$, resistive behavior of polysilicon with indicated quasi-linear operating regime (b).

resistance for polysilicon heater and temperature sensors had a non-linear behavior. Deviation from linearity of $\mathrm{R}(\mathrm{T})$ for polysilicon is a result of the complicated conductivity nature. The electron-hole exchange process occurs not only within the grains but also along the grain boundaries [108]. The TCR of polysilicon resistors integrated in various CMOS/MEMS based microsystems can be controlled in the range from $-1 \times 10^{-3}$ [109] to $1.1 \times 10^{-3} /{ }^{\circ} \mathrm{C}[110]$ and depends on the doping level.

Drawbacks of polysilicon thin films implemented as resistive temperature sensors shall also be mentioned. From wafer to wafer devices exhibited a slightly different TCR. This can be attributed to the structural properties of polysilicon as well as a minor deviation in the conditions (such as a maximum beam current) during the ion implantation. The TCR may also alter with time, therefore (re-)calibration of each device is advisable. Before direct utilization, polysilicon thin film resistors have to be subjected to heating ramps or high current stress to stabilize its resistive properties [111]. To improve the stability of polysilicon resistors, additional studies should be undertaken on the resistive behaviour of polysilicon at different doping levels, development of linerization models and drift compensating readout systems. Despite all these challenges polysilicon still provides the best compatibility with CMOS processing.

\section{Resistive measurements of polysilicon on fused silica}

In Figure 3.4a a measurement of the sheet resistance of polysilicon (highly doped with $\mathrm{B}^{+}$) deposited on fused silica is presented. The temperature was ramped between 50 and 200, 400, 600 and $800{ }^{\circ} \mathrm{C}$. After each heating ramp sufficient time was given to stabilize the temperature at $50{ }^{\circ} \mathrm{C}$. The material exhibited a positive TCR across the whole temperature range. The measured resistance was reversible only after the first two ramping cycles, thus, up to $400{ }^{\circ} \mathrm{C}$. In fact, above $500{ }^{\circ} \mathrm{C}$ the material showed unstable behaviour: the heating and cooling curves deviated and after cooling down a higher resistance value was observed with respect to the previous ramp. The reason for this may be attributed to a rearrangement in grains and grain boundaries. To stabilize the resistive behavior, the samples need to be subjected to additional temperature rampings. For 


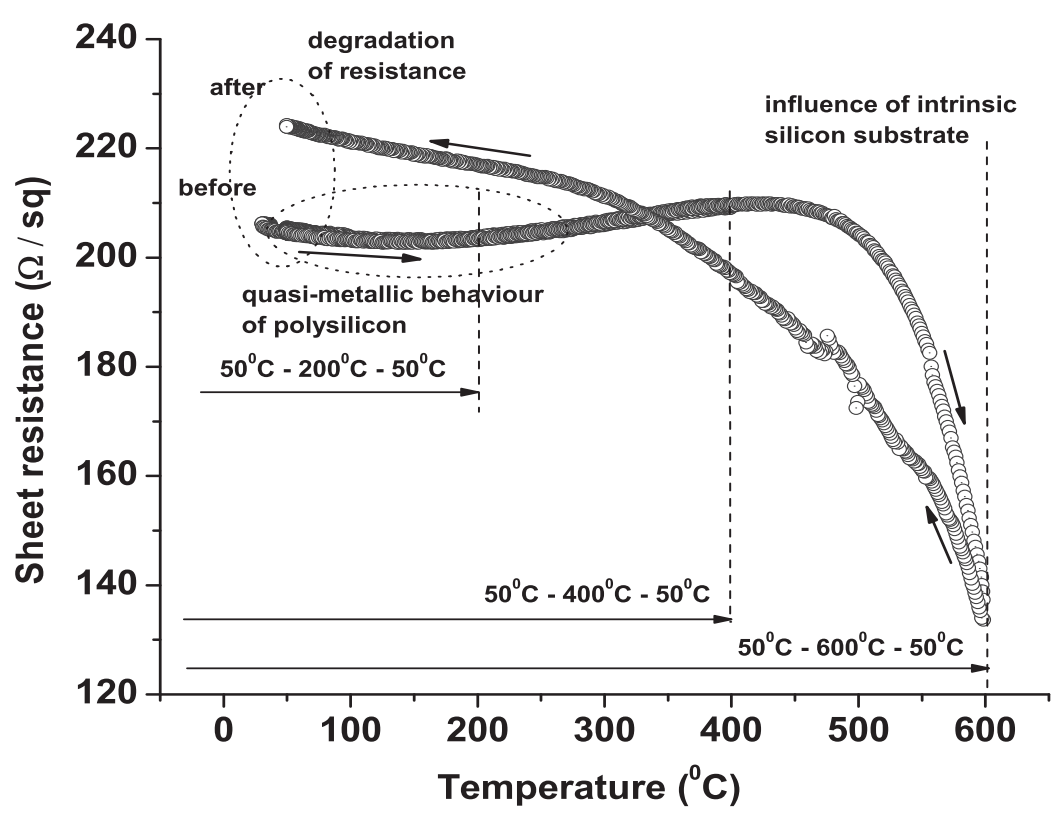

Figure 3.5: The resistance of thin polysilicon films deposited on Si/SiRN in time under temperature cycling conditions between 50 and $200,400,600$ and $800^{\circ} \mathrm{C}$.

the study of propane oxidation this was not required, we did not intend to exceed $500{ }^{\circ} \mathrm{C}$ during the device operation. Higher operational temperatures may lead to a poor relative to a baseline stability of R(time) for polysilicon resistors. During switching between the gases a new baseline was monitored. The sensor response was compared to a new set value for the baseline. In Figure 3.4b a typical temperature dependence of the sheet resistance for the processed polysilicon thin films is shown. A TCR of about $3.24 \times 10^{-4} /{ }^{\circ} \mathrm{C}$ was extracted in the quasi-linear resistive range between 300 and $500{ }^{\circ} \mathrm{C}$ (see Equations 3.4 and 3.5). We selected this range as a working regime in our study.

\section{Resistive measurements of polysilicon on $\mathrm{Si} / \mathrm{SiRN}$}

In Figure 3.5 the temperature dependence of the resistance for polysilicon (highly doped with $\mathrm{B}^{+}$) deposited on Si passivated with SiRN is presented. At elevated temperatures SiRN exhibited conductive properties. This resulted in a shortcut to the intrinsic silicon substrate, parallel to the polysilicon thin film. Thus, the observed rapid decrease in the resistance above $400{ }^{\circ} \mathrm{C}$ was attributed to the silicon substrate. At lower temperature, however, we saw the same trend as in Figure 4a: during the first two cycles, i.e up to $200{ }^{\circ} \mathrm{C}$ and $400{ }^{\circ} \mathrm{C}$, the material was stable. Its properties were reversible and no hysteresis was observed. However, once exceeding $450-500{ }^{\circ} \mathrm{C}$ the material underwent irreversible changes in the resistance.

\section{Stability test}

This test was performed with thin films that were already exposed to temperatures up to $800{ }^{\circ} \mathrm{C}$. In Figure 3.6 the resistance of polysilicon thin films is shown. The temperature was ramped between 50 and $400{ }^{\circ} \mathrm{C}$ and stabilized after each heating ramp at $50{ }^{\circ} \mathrm{C}$. The wafer was kept for $5 \mathrm{~min}$ at $400{ }^{\circ} \mathrm{C}$ during the first cycle. For each next cycle the annealing time was increased for $5 \mathrm{~min}$. The resistance was monitored in time to verify how the resistive properties of polysilicon thin films altered after a number of temperature ramps.

The scaling of the measured resistance values for 2 to 7 cycles up to $400{ }^{\circ} \mathrm{C}$ revealed that once a device was exposed at $400{ }^{\circ} \mathrm{C}$ its resistive properties were stabilized. After the first cycle the resistance was altered the 


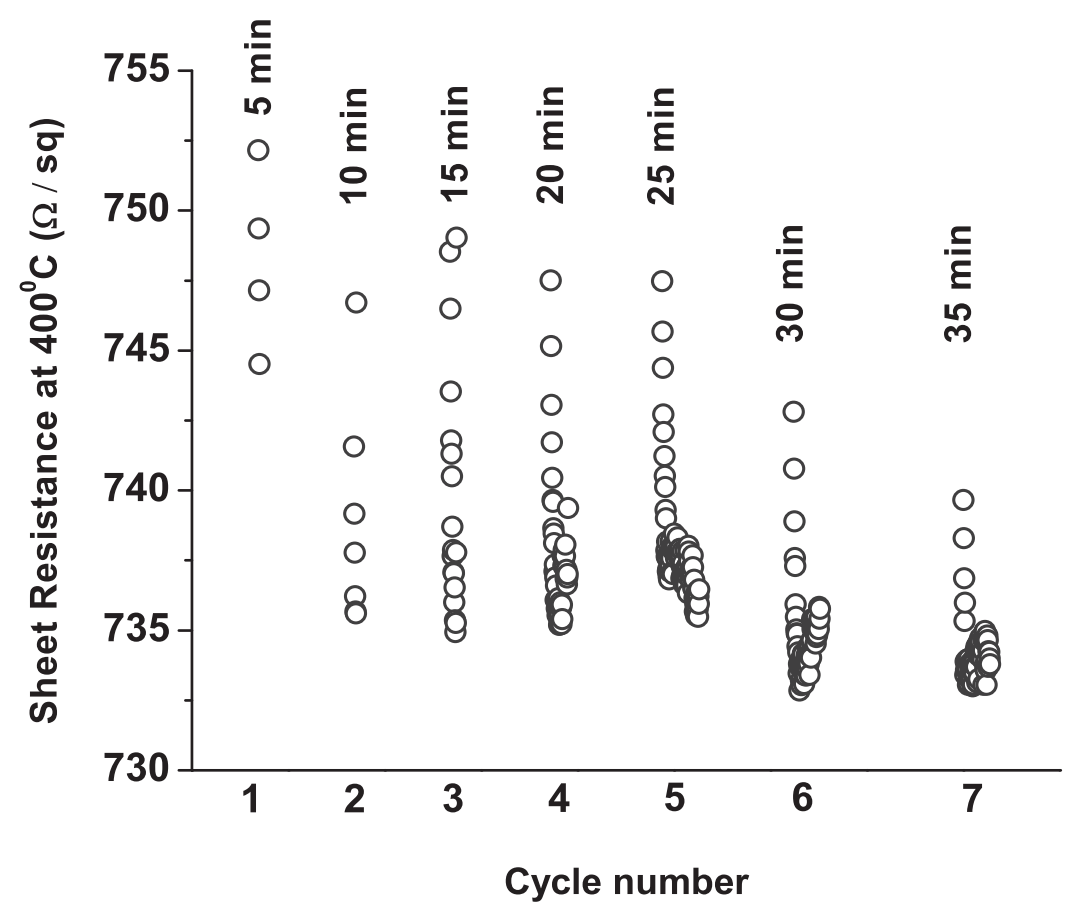

Figure 3.6: The stability of polysilicon thin films at 50 to $400^{\circ} \mathrm{C}$ temperature cycles with increasing annealing times in 5 min each cycle.

most. Although resistive drift may result in a poor relative to baseline stability, an absolute stability remained acceptable for measurements.
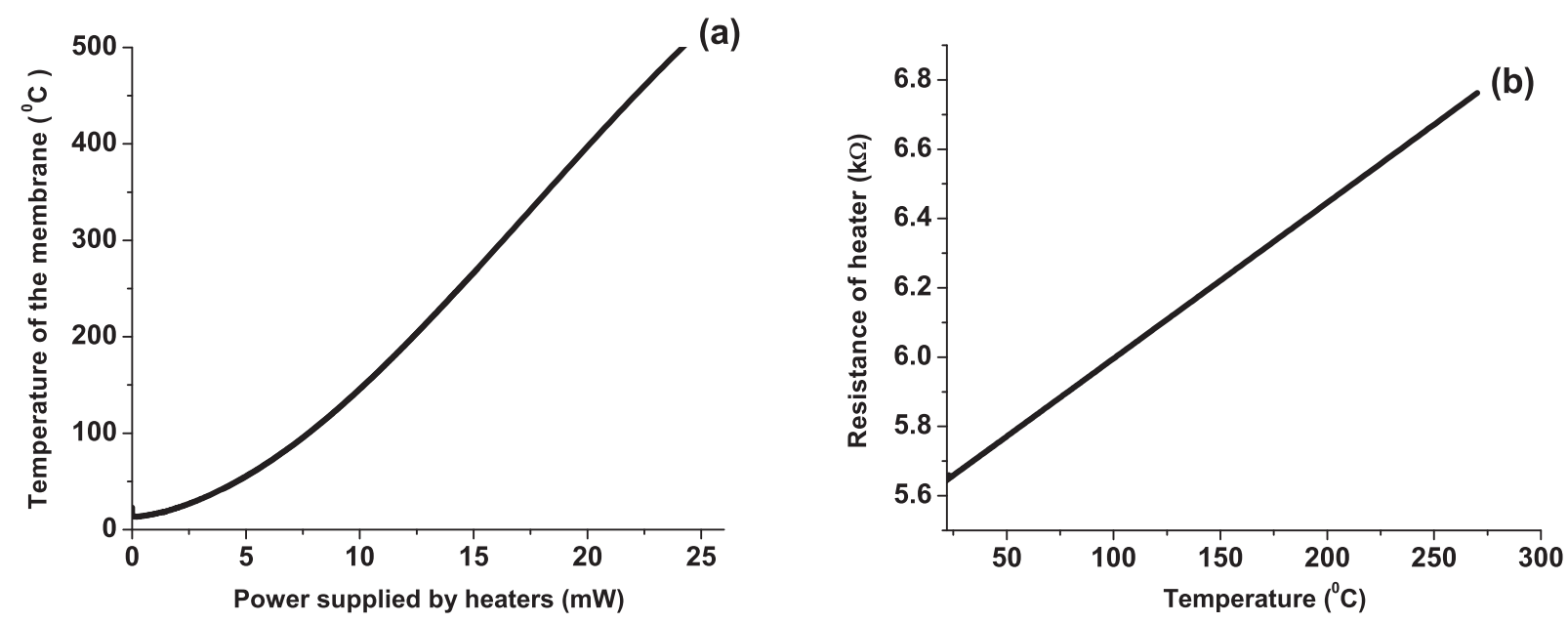

Figure 3.7: The thermo-electric properties of the sensor device: (a) temperature of the active area depending on power supplied to the integrated heaters, (b) temperature dependence of resistance for the polysilicon heater. 


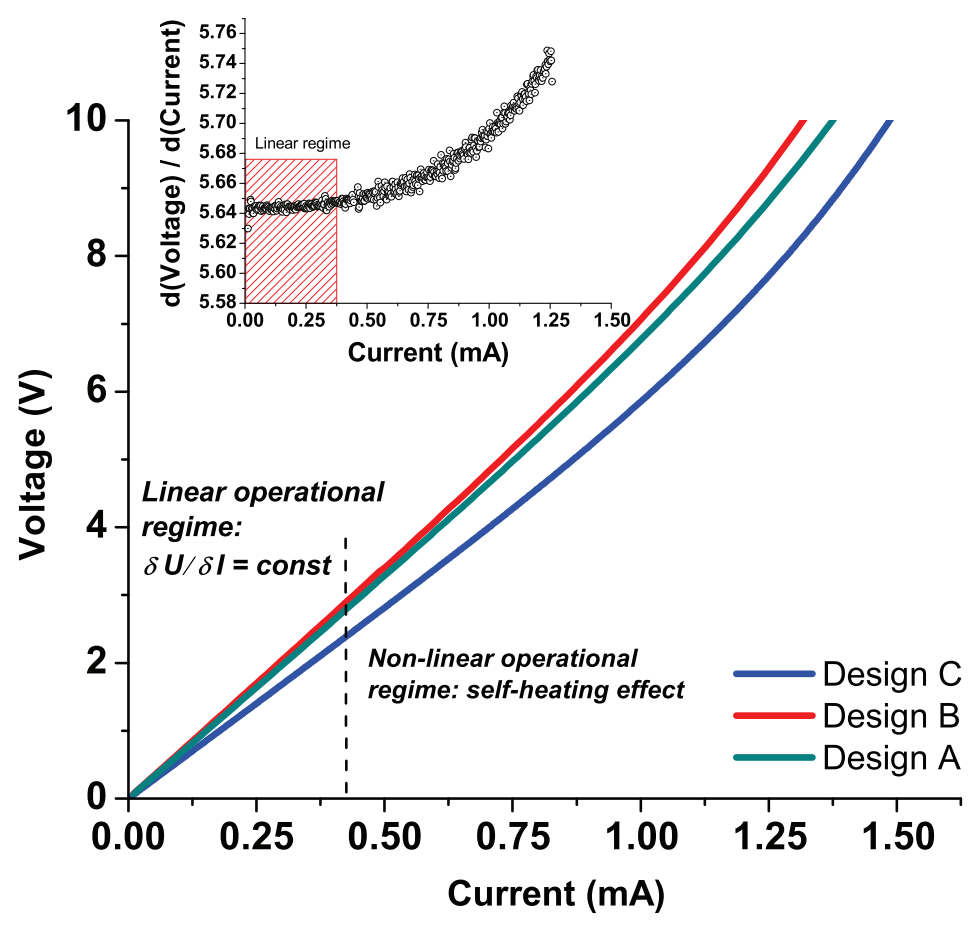

Figure 3.8: IV measurements (a) and indicated linear operation regime for the polysilicon thermistor $(b)$.

\subsubsection{Detection of reaction heats}

In Figure 3.7a the dependence of the power dissipated by the integrated heaters on the temperature of the active area of the sensor is shown. To operate the sensor within the operating temperatures a power of about 15 $20 \mathrm{~mW}$ was required. This was a reasonable value in terms of a minimization of the consumed power. In Figure $3.7 \mathrm{~b}$ the temperature dependence of the resistance for the polysilicon heater is shown. A quasi-linear profile was observed up to $300{ }^{\circ} \mathrm{C}$. A TCR of about $7 \times 10^{-4} /{ }^{\circ} \mathrm{C}$ was extracted. This is slightly higher compared to nonpatterned polysilicon thin films which exhibited the TCR of $3.24 \times 10^{-4} /{ }^{\circ} \mathrm{C}$. We also identified an operational regime for the temperature sensor read-out to minimize the effects of noise and self-heating (see Figure 3.8).

A typical result from the propane oxidation experiment is shown in Figure 3.9. The response time to catalytic reaction was within $2 \mathrm{sec}$ and the signal was completely reversible. Based on the TCR the temperature increase due to the reaction was estimated to be within $1{ }^{\circ} \mathrm{C}$ at concentrations below LEL ( 2.1 vol \% for propane).

The response time of the temperature sensor itself to an external heat excitation was estimated to be in a few millisecond range which made the analysis of fast catalytic reactions possible. This was also demonstrated in the past for the catalytic microreactors comprising the integrated heaters [112].

In Figure 3.10 a similar, long-term test is indicated. The sensor exhibited reproducible signal after $8 \mathrm{hr}$ of exposure to propane pulsing.

Measurements using a blank sensor without catalyst did not show any change in resistivity when switched between propane/ $\mathrm{N}_{2}$ and pure $\mathrm{N}_{2}$ at concentrations of propane below $1 \%$. Thus, the detected peaks were due to reaction heats and not due to the thermal conductivity difference between propane/ $\mathrm{N}_{2}$ and pure $\mathrm{N}_{2}$. The concentration of propane in the outlet of the sensor was monitored using the micro-GC. The quantitative analysis confirmed that a part of propane was catalytically converted. In Figure 3.11 the dependence of the normalized sensor signal and temperature increase due to the reaction on the power supplied by the heater is shown. Increase of the supplied power led to a subsequent temperature rise. Higher temperature generally speeds up the reaction due to the exponential dependence of the reaction rate on temperature. This results in a 


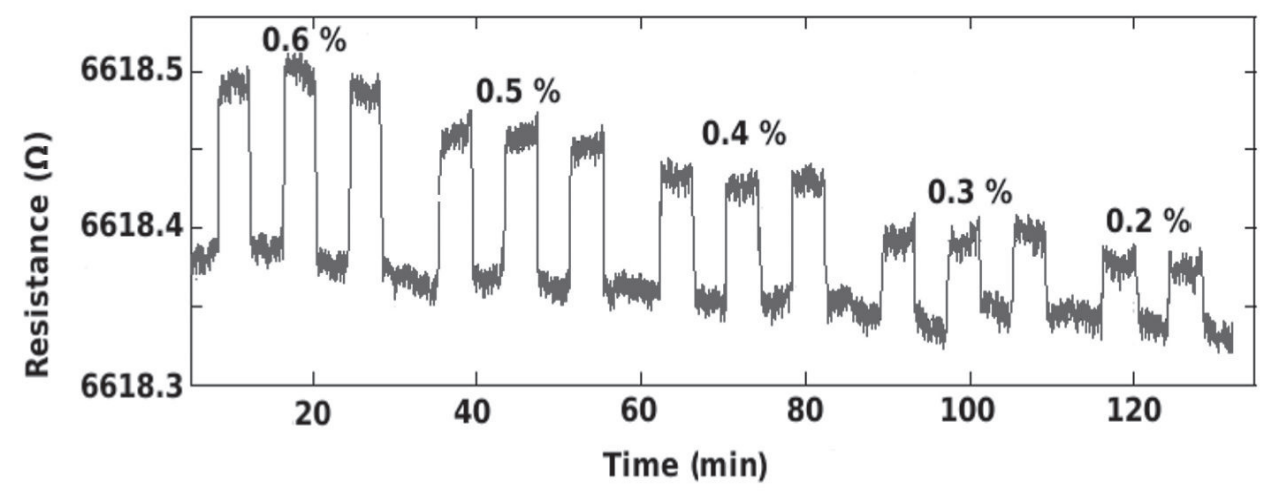

Figure 3.9: The sensing behaviour upon exposure to pulses of propane in air. The propane concentrations are indicated, the carrier gas is nitrogen. Total flow rate is 3 sccm and the supplied power is ca $8 \mathrm{~mW}$.

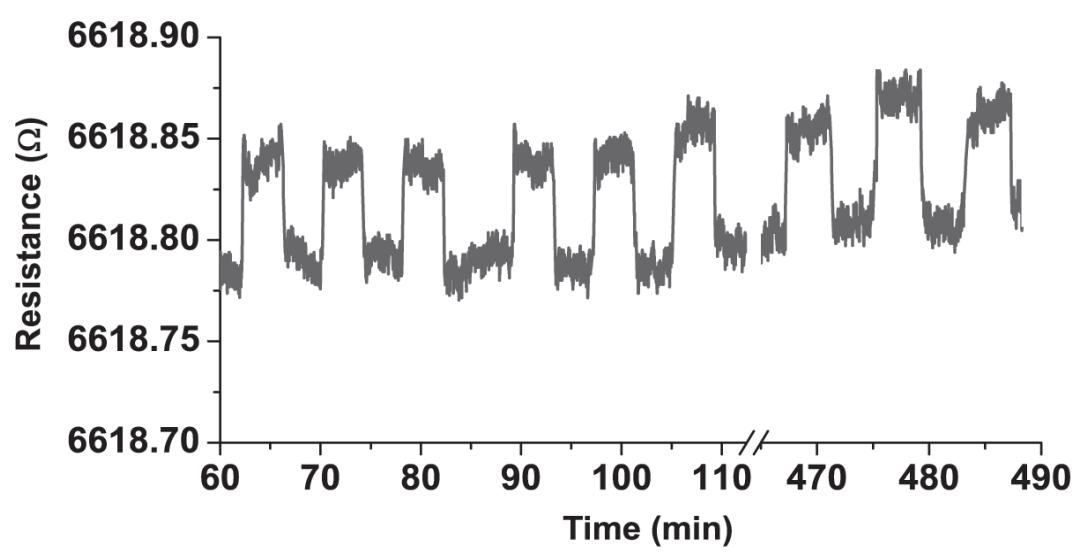

Figure 3.10: The sensing behaviour upon exposure to $0.3 \%$ propane in air during $8 \mathrm{hr}$. Total flow rate is 3 sccm and the supplied power is ca $8 \mathrm{~mW}$.

higher catalytic conversion and an increased heat generation. The more power was supplied to the system the higher the observed signal. On the other hand, this also increased the energy consumption of the system and could lead to a faster deactivation of the catalyst due to sintering and other deactivation processes.

Figure 3.12a shows the resistive responses upon exposure to propane at different flow rates. The concentration was kept at $0.8 \%$ of propane in air, the flow was varied from 1 to $3.6 \mathrm{sccm}$. The higher the flow rate, the faster the inset of the reaction occured due to the void space in the tubings. Minimized length and inner diameter of the connecting tubings, higher flow rates and minimized dimensions of the microfluidic channel resulted in a fast response. However, the height of the peaks remained constant at different flow rates. The measured time in response to the gas pulsing was within 2 seconds.

In Figure 3.12b the dependence of the conversion of propane at different gas flow rates is shown. The conversion values $(X)$ were calculated from the Equation 3.7, where $\mathrm{c}_{b e f o r e, r}$ and $\mathrm{c}_{a f t e r, r}$ denote concentration of propane 


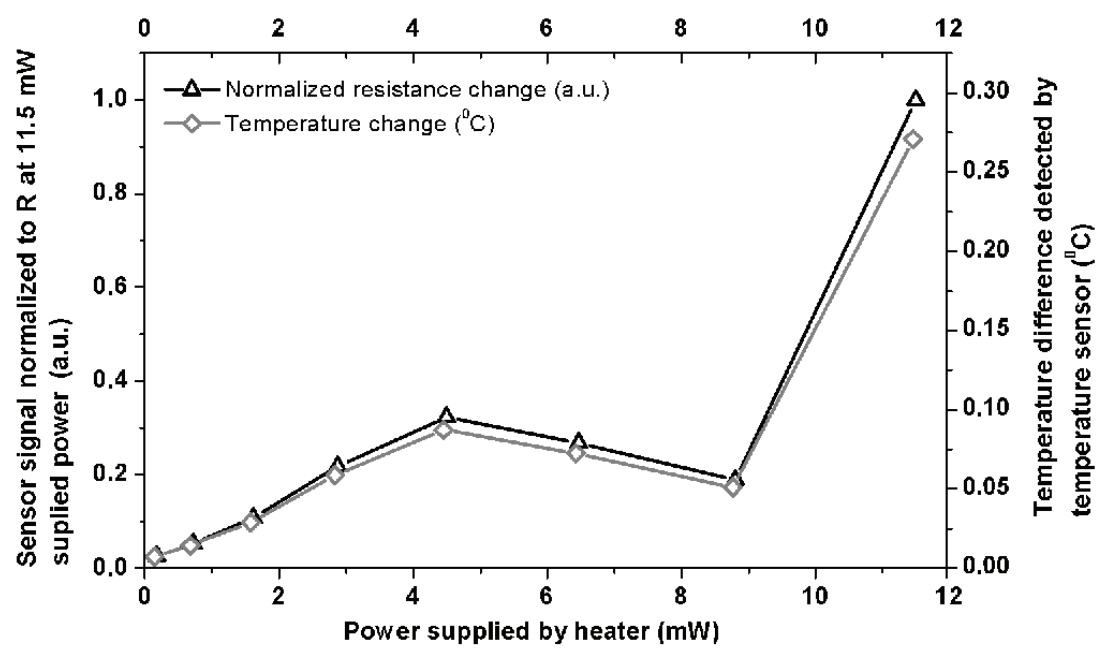

Figure 3.11: The dependence of the normalized sensor signal and subsequent temperature rise on the supplied power at total flow rate of $3 \mathrm{sccm}$.
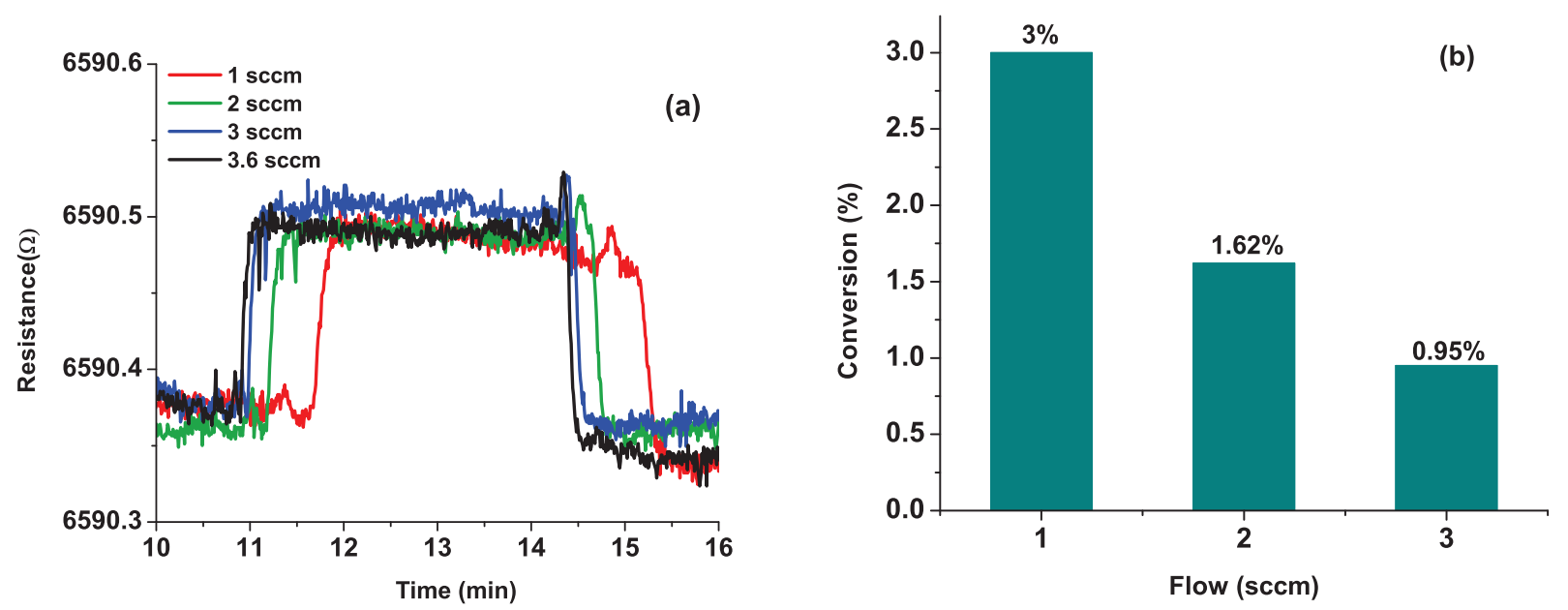

Figure 3.12: The sensor response upon exposure to $0.8 \%$ of propane in air at 1,2, 3 and 3.6 sccm (a) and conversion of propane at 1,2 and $3 \operatorname{sccm}(b)$.

in air measured by the Micro GC before and after the reaction.

$$
X=\frac{c_{\text {before }, r}-c_{a f t e r, r}}{c_{\text {before }, r}}
$$

The higher the flow rate was set, the less the measured values of conversion. This can be explained by the reduced residence time, i.e. the time available for the propane to react on the catalyst. In case of $3 \mathrm{sccm}$ the conversion was three times lower than for $1 \mathrm{sccm}$. The flow rate was, however, three times higher. This means that per time interval the same amount of molecules was converted and the same amount of heat was released. A cooling effect due to higher flow rates could not be observed at these concentrations.

Finally, the sensor response upon exposure to propane of different concentrations is presented in Figure 3.13 for devices with a thickness of the SiRN membrane of 200 and $400 \mathrm{~nm}$. For the thinner membrane the signal was twice as high due to better thermal isolating properties. A further reduction of the membrane thickness or 
realization of fully-suspended (isolated by air) devices can improve the performance even more. However, this also reduces the mechanical stability of the sensor.

The reaction was performed at concentrations of propane ( 0.01 to $0.8 \mathrm{vol} \%)$. Reproducible measurements were possible for propane concentrations down to $0.1 \%$. For lower concentrations the signal-to-noise ratio decreased

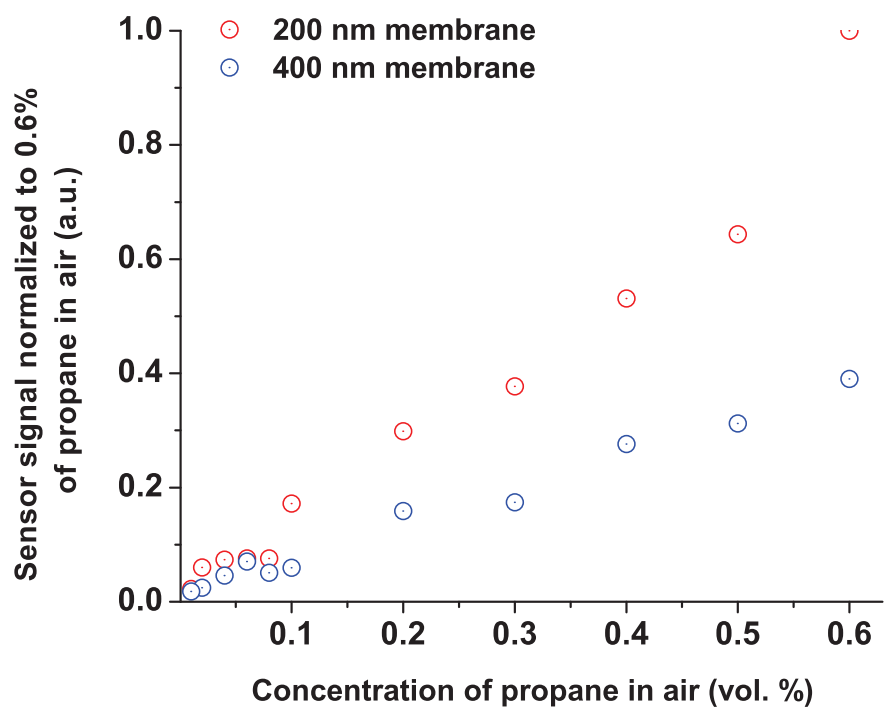

Figure 3.13: The normalized sensor response: comparison of the sensor device with SiRN membranes of 200 and $400 \mathrm{~nm}$ thickness. The concentration varied between 0.01 and 0.6 vol.\% of propane in air at a total flow rate of $3 \mathrm{sccm}$.

below 4 making precise measurement of concentration challenging. Observed sensing behaviour corresponded to recently reported by Bársony and co-workers [12]. Based on the experimental results we concluded that the developed microcalorimetric sensor can be successfully used for the detection of hazardous gases below LEL. The detection limit can be further shifted to lower ppm range if the temperature sensors with a higher TCR were used.

The ultimate goal of the future study is a detection of individual gases in a mixture. This can be achieved by measuring the reaction heat and the temperature at which reaction starts [113], [114]. These experiments are on-going.

\subsection{Conclusions}

In this work we presented the fabrication, characterization and testing of a low-cost, low-power, CMOScompatible microcalorimetric sensor for detection of reaction heats.

The construction of the calorimetric sensing system based on extrinsically-doped polysilicon resistive heaters and temperature sensors has been demonstrated. The compatibility with Si technology and the chemical inertness at elevated temperatures makes polysilicon attractive for integration into microreactors and sensors. However, non-linearity of its resistive properties and drift still offer challenges on a way to its successful implementation as a functional sensing material.

We demonstrated the detection of reaction heats for a model reaction - the catalytic oxidation of propane in air. The reaction was performed over the thin film of Pd catalyst at concentrations of propane (0.01 to 0.8 vol \%) which is significantly below the low explosion level of propane. The sensor exhibits immediate and reversible response upon exposure to propane in air. The short response times allow this detection mechanism to be ap- 
plied in transient studies of chemical reactions, such as differential scanning microcalorimetry.

The sensor can be applied for the detection of reaction heats and kinetic analysis, where commonly higher concentrations of reactive components are used, which will lead to stronger heat release. Besides that it can be utilized as a safety sensor for the detection of propane leaks. In addition, immobilizing of a highly active catalyst with a high surface area on the sensor can significantly increase the sensitivity. 


\section{4 \\ Development of titanium silicide - boron-doped polysilicon resistive temperature sensors}

Thin films of titanium silicide ( $\mathrm{TiSi}_{2}$ ) formed on heavily boron doped polycrystalline silicon (poly-Si/B ${ }^{+}$) were for the first time applied for resistive temperature sensing. The temperature sensors exhibited a high temperature coefficient of resistance (TCR) of $3.8 \times 10^{-3} \rho C$, a linear dependence of resistance on temperature and an excellent thermal and electrical stability up to $800{ }^{\circ} \mathrm{C}$. This work discusses the fabrication method, and the morphological and the electrical characterization of the TiSi $2 /$ poly-Si thin film resistors throughout the stages of its formation.

${ }^{*}$ Published as: E.Vereshchagina, R.A.M.Wolters, J.G.E.Gardeniers, Development of titanium silicide - boron-doped polysilicon resistive temperature sensors, J. Michromech. Microeng. 21105022. 


\subsection{Introduction}

Accurate control and measurement of temperature is an essential requirement in various sensor and microfluidic applications. The precise measurement of reaction heats by microcalorimetry is used as a transduction mechanism for the detection of combustible gases, with typical examples [11], [12], [13], [71]. Also, high-throughput, sensitive thermal analysis of bio-chemical interactions is an important application, and was achieved using microfluidic calorimeters as demonstrated by W.Lee and co-workers [35]. In microreactors used for production or analysis of (bio-)chemical compounds [115], [116], and in heterogeneous catalysis research [117] good control over the temperature is necessary because of the exponential dependence of reaction rates.

For many of the commonly used resistive materials, e.g. metallic thin films and semiconductors, poor sensitivity and stability at high temperatures and incompatibility with integration on a silicon chip still belong to the major drawbacks. Additionally, microsystems for chemical analysis require materials that are chemically resistant towards the reactive components and the reaction products: on one hand to prolong the life-time of a thermistor, and on the other hand to ensure zero blank activity of the device during operation. Moreover, commonly used metal thin film resistors, when not passivated, can act as a very reactive catalyst, thereby actuating (undesirable) reactions. These drawbacks bring a demand for new emerging materials that meet requirements of technological compatibility as well as exhibit excellent thermo-electrical properties and stability at high temperatures.

There is a wide range of materials that can be successfully applied in thermistors [118]. From the candidates for high temperature sensing we selected $\mathrm{TiSi}_{2}$ on heavily boron $\left(\mathrm{B}^{+}\right)$doped poly-Si. To the best of our knowledge, this is the first time that the combination of these two materials was employed for resistive temperature sensing up to $800{ }^{\circ} \mathrm{C}$. Other types of silicides for heating and temperature sensing purposes are tantalum silicide $\left(\mathrm{TaSi}_{2}\right)$, cobalt silicide $\left(\mathrm{CoSi}_{2}\right)$ [28], molybdenum silicide $\left(\mathrm{MoSi}_{2}\right)$ [119], nickel silicide (NiSi) [120] and platinum silicide (PtSi)[121].

$\mathrm{TiSi}_{2}$ is commonly used in the semiconductor industry to reduce parasitic series resistance and contact resistance between metallization and device [122], [123], [124], [125]. Therefore it is a promising material for accurate temperature sensing. It has a low resistivity, high TCR, good thermal, chemical and electrical stability, low silicide-to-silicon contact resistance and established compatibility with silicon technology [126], [127].

An objective of this work is to evaluate the feasibility of $\mathrm{TiSi}_{2} /$ poly-Si thin films as resistive temperature sensors. We will discuss here the technological aspects of the silicide formation, the integration in a Si-based process and the morphological and electrical characterization. Realized structures exhibited a high TCR and excellent thermo-electrical stability and, are, therefore, suitable for integration in temperature-assisted lab-on-a-chip microsystems.

\subsection{Choice of a material for temperature sensing}

\subsubsection{Requirements of the sensor material}

The material for temperature sensing should satisfy a number of important constraints imposed by the manufacturing technology and the field of potential application:

- Linear change of resistance in a wide temperature range;

- High and stable TCR;

- Short- and longterm stability of electrical characteristics;

- Chemical inertness at elevated temperatures;

- Compatibility with established CMOS processing facilities;

- Potential applicability in MEMS/NEMS; 
Table 4.1: Comparison of thermo-electrical properties of thin silicide films.

\begin{tabular}{lccccc}
\hline Silicide & $\begin{array}{c}\mathrm{T}_{\text {form }} \\
\left({ }^{\circ} \mathrm{C}\right)^{a, b}\end{array}$ & $\begin{array}{c}\text { Resistivity } \\
(\mu \Omega \cdot \mathrm{cm})^{b}\end{array}$ & $\begin{array}{c}\mathrm{TCR} \times 10^{-3} \\
\left({ }^{\circ} \mathrm{C}^{-1}\right)^{a, c, d, e}\end{array}$ & R-T curve ${ }^{e}$ & $\begin{array}{c}\mathrm{T}_{\max } \text { on Si } \\
\left({ }^{\circ} \mathrm{C}\right)^{f}\end{array}$ \\
\hline \hline $\mathrm{TiSi}_{2}$ & $700-900$ & $13-20$ & $3.8-4.3$ & Linear & 950 \\
$\mathrm{TaSi}_{2}$ & 650 & $50-60$ & $1.57-1.75$ & Non-linear & 1000 \\
$\mathrm{CoSi}_{2}$ & $600-700$ & $14-20$ & $3.62-4.33$ & Linear & 950 \\
$\mathrm{MoSi}_{2}$ & 525 & $100-120$ & 2.58 & Linear & 1000 \\
$\mathrm{NiSi}$ & $400-600$ & $14-20$ & 4.3 & Linear & 850 \\
$\mathrm{PtSi}$ & $300-600$ & $28-35$ & - & - & 750 \\
\hline \multicolumn{5}{c}{${ }^{a}[127],{ }^{b}[123],{ }^{c}[28],{ }^{d}[119],{ }^{e}[120],{ }^{f}[124]$} \\
\end{tabular}

Platinum (Pt, TCR $=3.9 \times 10^{-3} /{ }^{\circ} \mathrm{C}$ ) might be a preferred material for temperature sensing [100], [101]. However, thermistors based on Pt often require non-standard, low-temperature processing which is not compatible with processing on poly-Si. Moreover, during operation above $550{ }^{\circ} \mathrm{C} \mathrm{Pt}$ thin films may agglomerate and alter the resistive properties. Pt has poor adhesion to $\mathrm{Si}, \mathrm{SiO}_{2}, \mathrm{Si}_{x} \mathrm{~N}_{y}$ and, therefore, an adhesion layer is required. Most of the issues related to Pt reliability are due to the degradation of the Pt adhesion layer at elevated temperatures [104].

Thin films of poly-Si do not require an adhesion layer and a high electrical stability is reported [102]. Highly doped poly-Si can be used for temperature sensing in a limited temperature range and at high dopant concentrations a positive TCR is observed [71]. However, for these high dopant concentrations, the non-linearity of electrical properties, the relatively low TCR and the potential to resistive drift may limit its application. In addition, above $450-500{ }^{\circ} \mathrm{C}$ effects of grains and grain boundaries movement lead to deviation of resistance and, therefore, burn-in procedure is required. In Table 4.1 the thermo-electrical properties of commonly utilized thin silicide films are summarized. NiSi and PtSi do not match the criteria of high-temperature stability. The resistance of $\mathrm{TaSi}_{2}$ changes non-linearly with temperature and along with $\mathrm{MoSi}_{2}$ it exhibits lower TCR values compared to other silicides. Based on the above mentioned design constraints, reported electrical properties and desirable compatibility with standard CMOS processing, $\mathrm{TiSi}_{2}$ or $\mathrm{CoSi}_{2}$ is the best option for temperature sensing.

\subsection{2 $\mathrm{TiSi}_{2}$ /poly-Si thin films for temperature sensing}

We selected $\mathrm{TiSi}_{2}$ on heavily $\mathrm{B}^{+}$doped poly-Si as sensor material because, unlike the commonly used $\mathrm{Pt}$ and poly-Si thin films, it meets all criteria mentioned above. The $\mathrm{TiSi}_{2}$ was formed by reaction of Ti with poly-Si during rapid thermal annealing (RTA). Conventional thermal annealing (CTA) is not practical because Ti is prone to oxidation. During a first annealing step at a temperature of $700-750{ }^{\circ} \mathrm{C}$ the metastable $\mathrm{C} 9 \mathrm{TiSi}_{2}$ phase is formed. In a second annealing step, at a temperature of $850-900{ }^{\circ} \mathrm{C}$, the stable low resistive $\mathrm{C} 54 \mathrm{TiSi}_{2}$ phase is obtained. A detailed description on the kinetics of this transformation to the C54 phase can be found elsewhere [128]. The stable low-resistive C54 phase of $\mathrm{TiSi}_{2}$ is the desirable material for sensing applications in the present study. It exhibits metallic-like behavior with a resistivity $(\rho)$ in the range of $13-20 \mu \Omega \cdot \mathrm{cm}$ and a TCR in the range of $(3-3.6) \times 10^{-3} /{ }^{\circ} \mathrm{C}[129]$. In $\mathrm{TiSi}_{2} /$ poly-Si thin films the electrical properties are governed by the $\mathrm{TiSi}_{2}$. We concluded this based on the fact that $\mathrm{TiSi}_{2}$ has lower resistance and higher TCR compared to poly-Si. This makes $\mathrm{TiSi}_{2} /$ poly-Si system suitable for use in resistive temperature sensing. By nature, $\mathrm{TiSi}_{2}$ is covered with a layer of native oxide $\left(\mathrm{SiO}_{2}\right)$. It can be oxidized further to a thicker $\mathrm{SiO}_{2}$ layer at the cost of the underlying poly-Si layer. The $\mathrm{TiSi}_{2}$ film itself remains undisturbed.

The sensing material should not initiate any (undesirable) reactions in the system, i.e. stay chemically inert at elevated temperatures, such that only the reactions of interest give a significant signal. $\mathrm{TiSi}_{2}$ is a chemically stable material and reported to resist temporally immersion in a potassium-hydroxide (KOH) solution [130]. 
It also can be used as a masking material for silicon surface [131] and bulk [132] micro-machining. $\mathrm{TiSi}_{2}$ is formed where $\mathrm{Ti}$ is in contact with $\mathrm{Si}$. Therefore, the formation of silicides will only occur on predefined Sibased structures. This is commonly addressed as the self-aligned silicide (SALICIDE) technology [122].

The compatibility with $\mathrm{Si}$ technology allows the realization of miniature sensor devices with advanced functionality at reduced costs in Si IC manufacturing facilities. The good thermal stability of $\mathrm{TiSi}_{2}$ up to $800{ }^{\circ} \mathrm{C}$ permits high temperature processing, such as temperature-assisted anodic bonding. This facilitates merging of the silicon-based sensors with MEMS/NEMS technology.

In addition, the $\mathrm{TiSi}_{2}$ has unique light-absorptive properties and, therefore, can be attractive for the lab-on-achip devices utilizing optical detection [133].

\subsubsection{The technological aspects of $\mathrm{TiSi}_{2}$ integration}

In this section we discuss the issues to be considered during the fabrication and integration of the $\mathrm{TiSi}_{2} / \mathrm{poly}-\mathrm{Si}$ temperature sensors in the Si-based process. Below the factors that influence the thermo-electrical properties of the formed $\mathrm{TiSi}_{2}$ on poly-Si are given [134]:

- Annealing conditions (ambient gas, temperature, time);

- Properties of the poly-Si (grain size distribution and doping level);

- Cleanliness of the Ti/poly-Si interface prior to the formation of the $\mathrm{TiSi}_{2}$;

- Thickness of the formed $\mathrm{TiSi}_{2}$;

- Sensitivity of the grain boundaries to the high temperature anneals;

- Oxidation behavior of the $\mathrm{TiSi}_{2} /$ poly-Si stack.

The annealing temperatures are known from the studies in which, by means of the in-situ 4-point-probe measurements the resistivity and the phase changes from Ti to $\mathrm{C} 49-\mathrm{TiSi}_{2}$ and further to $\mathrm{C} 54-\mathrm{TiSi}_{2}$ were monitored during the annealing. From such measurements is known that the C49-C54 phase change occurs around 850$900{ }^{\circ} \mathrm{C}$.

Commonly, argon (Ar) or nitrogen $\left(\mathrm{N}_{2}\right)$ is used as annealing ambient. Annealing in Ar leads to the formation of $\mathrm{TiSi}_{2}$ at the risk of an excessive lateral growth of silicide over the neighboring dielectric. This can cause problems in CMOS applications limited to the nanoscale dimensions of the Si gates. Therefore, to suppress a lateral diffusion of $\mathrm{Si}$ into $\mathrm{Ti}$, annealing in $\mathrm{N}_{2}$ ambient is preferred.

The silicide formation for $\mathrm{Ti}$ on $\mathrm{Si}$ in $\mathrm{N}_{2}$ ambient results in the simultaneous formation of $\mathrm{TiN}$ and $\mathrm{TiSi}_{2}$ on $\mathrm{Si}$ areas, whereas only TiN is formed on the dielectric regions. This prevents lateral growth of the silicide. The TiN layer can be further etched to release silicide.

The substrate and dopant in the underlying poly-Si have great impact on grain size, resistivity and the thermal stability of the formed silicide layer. This was demonstrated for Co/poly-Si resistive lines by Y.M.Chen and co-workers [135]. The presence of the dopant also impacts silicide properties. It has been reported that n-type dopant leads to a modification of the grain structure of poly-Si, and that doping of poly-Si with As or P greatly enhances the thermal stability [136].

The surface of the poly-Si should remain particle- and water-free prior to the sputtering of Ti. Besides formation of a native oxide during CTA (performed for the activation of $\mathrm{B}$ in poly-Si), a thicker under stoichiometric boron oxide $\left(\mathrm{B}_{2} \mathrm{O}_{5}\right)$ can be formed at the interface of poly-Si / $\mathrm{SiO}_{2}$. Therefore, a standard oxide etch must be done to ensure an oxide-free surface.

Karlin and co-workers pointed out that the thickness of the $\mathrm{TiSi}_{2}$ plays an important role. They report on the improved thermal stability as the thickness of the $\mathrm{TiSi}_{2}$ was doubled [136]. By applying a thicker Ti layer a thicker silicide layer can be obtained with the enhanced consumption of the underlying poly-Si. This is reflected in the total resistance of $\mathrm{TiSi}_{2} /$ poly-Si stack.

The poly-Si/ $\mathrm{B}^{+}$combination is known for a lower sensitivity to oxidation, which is an advantage. On the other 
Table 4.2: The annealing conditions in nitrogen and former gas and the measured values of sheet resistance.

\begin{tabular}{lccccc}
\hline & Ramp & Temperature & Duration & \multicolumn{2}{c}{ Sheet resistance $(\Omega / \mathrm{sq})$} \\
\cline { 5 - 6 } & $\left({ }^{\circ} \mathrm{C} / \mathrm{sec}\right)$ & $\left({ }^{\circ} \mathrm{C}\right)$ & $(\mathrm{sec})$ & $\mathrm{N}_{2}$ & $\mathrm{H}_{2} / \mathrm{N}_{2}$ \\
\hline \hline $1^{s t}$ anneal & 50 & 700 & 30 & 25.38 & 18.54 \\
$2^{\text {nd }}$ anneal & 50 & 900 & 30 & 11.78 & 5.75 \\
\hline
\end{tabular}

hand, at high temperatures atoms of $\mathrm{B}^{+}$can inhibit the formation of the $\mathrm{TiSi}_{2}$ due the formation of titanium diboride $\left(\mathrm{TiB}_{2}\right)$.

\subsection{Formation and characterization of the $\mathrm{TiSi}_{2}$ thin films}

\subsubsection{The silicidation procedure}

In this section we discuss the technological aspects of the $\mathrm{TiSi}_{2}$ formation. $\mathrm{TiSi}_{2}$ was formed by a two step temperature-assisted reaction of sputtered Ti and poly-Si. Prior to the manufacturing of the sensor prototype, the silicidation procedure was tested on two types of substrates:

- Si covered with $200 \mathrm{~nm}$ of Silicon Rich Nitride (SiRN);

- Fused Silica (FS) wafers.

The preparation procedure was as follows. Single crystal, p-type, $\{100\}$ oriented one-side polished Si wafers, $100 \mathrm{~mm}$ in diameter, $525 \mu \mathrm{m}$ thick and with a resistivity of $5-10 \Omega \cdot \mathrm{cm}$, were used. Prior to the deposition of $200 \mathrm{~nm}$ thick SiRN, the wafers were subjected to standard chemical cleaning with nitric acid and strip of a native oxide. The nitride was grown by Low Pressure Chemical Vapor Deposition (LPCVD) at $850{ }^{\circ} \mathrm{C}$ using $\mathrm{SiCl}_{2} \mathrm{H}_{2}$ and $\mathrm{NH}_{3}$ gases. For the electrical characterization of $\mathrm{TiSi}_{2}$ thin films above $250{ }^{\circ} \mathrm{C}$ we used doubleside polished FS wafers (see section 3.3) of Q1 quality with a diameter of $100 \mathrm{~mm}$ and a thickness of $525 \mu \mathrm{m}$, supplied by SCHOTT AG (Grünenplan, Germany). The Si/SiRN and FS wafers were subjected to a growth of $500 \mathrm{~nm}$ of LPCVD poly-Si at $620{ }^{\circ} \mathrm{C}$ from $\mathrm{SiH}_{4}$. Ion implantation of poly-Si was performed to define its sheet resistance $\left(\mathrm{R}_{\text {sheet }}\right)$ : $\mathrm{B}^{+}$with a dose of $5 \times 10^{15} \mathrm{ions} / \mathrm{cm}^{2}$ and an energy of $80 \mathrm{keV}$ was implanted into the poly-Si. Subsequently, CTA in $\mathrm{N}_{2}$ at $800{ }^{\circ} \mathrm{C}$ for $30 \mathrm{~min}$ was done to remove the damage in $\mathrm{Si}$ and to activate the $\mathrm{B}^{+}$. This resulted in a dopant concentration of about $10^{20} \mathrm{~B} / \mathrm{cm}^{3}$. The $\mathrm{R}_{\text {sheet }}$ of poly-Si on Si/SiRN was measured to be ca. $220 \Omega / \mathrm{sq}$. The $\mathrm{R}_{\text {sheet }}$ of poly-Si on FS was slightly lower compared to Si: ca. $190 \Omega / \mathrm{sq}$. These B-doped poly-Si films with $\mathrm{R}_{\text {sheet }}$ values in the range $190-220 \Omega /$ sq were used as a base layer for the formation of integrated $\mathrm{TiSi}_{2} /$ poly-Si resistive temperature sensors and poly-Si heaters. The presence of any n-type (As, P) or p-type (B) dopant in the poly-Si is generally known to slow down the transformation rate to the desirable $\mathrm{C} 54$ phase [122]. Considering this fact the annealing temperatures were selected as: $700{ }^{\circ} \mathrm{C}$ for the first anneal and $900{ }^{\circ} \mathrm{C}$ for the second anneal. The annealing was performed using a Solaris 150 Rapid Thermal Processing System (Surface Science Integration, El Mirage, AZ, USA). The heating and cooling rates were $50{ }^{\circ} \mathrm{C} / \mathrm{sec}$ and the wafer was kept at a set temperature for $30 \mathrm{sec}$. A flow of forming gas $\left(95 \% \mathrm{~N}_{2} / 5 \%\right.$ $\mathrm{H}_{2}$ ) was used to prevent oxidation. The experiments in pure $\mathrm{N}_{2}$ were also performed. The resistance of the layers obtained by annealing in $\mathrm{N}_{2}$ were higher than of samples annealed in the forming gas (see Table 4.2). This was ascribed to contamination by residual oxygen $\left(\mathrm{O}_{2}\right)$. It is recommended to keep the concentration of $\mathrm{O}_{2}$ in the annealing gas below $10 \mathrm{ppm}$. In practice, it is difficult to eliminate $\mathrm{O}_{2}$ completely in an atmospheric system. Prior to the deposition of the Ti films, wafers were chemically cleaned and dipped for $1 \mathrm{~min}$ in $1 \%$ hydrofluoric acid solution (HF).A $50 \mathrm{~nm}$ thick Ti was deposited at pressure of $0.066 \mathrm{mbar}$, in $148 \mathrm{sccm}$ Ar flow and power of $200 \mathrm{~W}$ using a home-built DC magnetron sputtering system. A pre-sputtering run for 20 min was performed to clean the Ti target before the actual deposition. The thickness of the Ti films was measured by a profilometer Veeco Dektak 8 (Veeco, Plainview, NY, USA). The measurements indicated a centersymmetric 
(a)

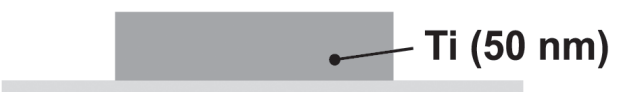

poly-Si $(500 \mathrm{~nm})$

(b)

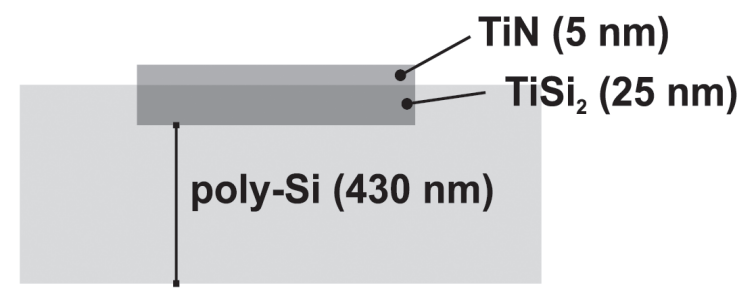

Figure 4.1: Schematic representation for the poly-Si uptake due to the silicidation process.

non-uniformity: a thicker film in the center and decreasing with ca. $10 \%$ towards the edges of the wafer. The non-uniformity of the Ti thickness should be taken into account during the fabrication because the thickness of the formed silicide layer and the phase formation rate are dependent on it. The surface roughness (Ra) of the Ti thin was $1.81 \mathrm{~nm}$ as measured using Veeco Dektak 8. After the deposition of Ti, wafers were placed in the RTA system. After flushing with forming gas, the first anneal was performed. A typical RTA run in forming gas consisted of a temperature ramp of $50^{\circ} \mathrm{C} / \mathrm{sec}$ up to $700{ }^{\circ} \mathrm{C}$, hold for $30 \mathrm{sec}$, and cooling at 50 ${ }^{\circ} \mathrm{C} / \mathrm{sec}$ rate down to room temperature. In Figure 4.1 we schematically illustrate the uptake of the poly-Si and formation of the $\mathrm{TiSi}_{2}$ and $\mathrm{TiN}$. The formation of $\mathrm{TiSi}_{2}$ proceeded by the consumption of the underlying poly-Si. Part of the Ti was consumed by the gas-phase nitridation to titanium nitride (TiN). High-Resolution Scanning Electron Microscopy (HRSEM) analysis revealed that during the reaction $60-70 \mathrm{~nm}$ of the poly-Si was consumed for the formation of about $25 \mathrm{~nm}$ of $\mathrm{TiSi}_{2}$. The cross-sectional SEM images of the TiSi $2 / \mathrm{poly}_{-} \mathrm{Si}$ stack are presented in Figure 4.2. Formation of the meta-stable C49 phase, the TiN and the stable C54 phase was confirmed by the 4-point-probe resistance measurements. During the first anneal Ti reacts with poly-Si to form $\mathrm{TiSi}_{2}$ and $\mathrm{TiN}$. However, Ti may also react with the $\mathrm{SiO}_{2}$ resulting in a metal-rich $\mathrm{Ti}_{5} \mathrm{Si}_{3}$ silicide compound. The unreacted $\mathrm{Ti}$, the formed $\mathrm{TiN}$ as well as the $\mathrm{Ti}_{5} \mathrm{Si}_{3}$ were etched for $10 \mathrm{~min}$ in a solution of hydrogen peroxide $\left(\mathrm{H}_{2} \mathrm{O}_{2}\right)$, ammonia and water (1:1:1). During the etching a color change was observed: from light-gold of TiN to grayish of $\mathrm{TiSi}_{2}$. After stripping of $\mathrm{TiN}$ the second anneal was performed at $900{ }^{\circ} \mathrm{C}$. The 4-point-probe measurements indicated a resistance drop from $46 \mu \Omega \cdot \mathrm{cm}$, for the C49 meta-stable phase, to 14 $\mu \Omega \cdot \mathrm{cm}$ for the stable C54-phase of $\mathrm{TiSi}_{2}$. The $\mathrm{R}_{\text {sheet }}$ resistance values are listed in Table 2 and correspond to $25 \mathrm{~nm} \mathrm{TiSi} i_{2}$ on $430 \mathrm{~nm}$ poly-Si. The silicide with $\mathrm{R}_{\text {sheet }}$ in order of $5-6 \Omega / \mathrm{sq}$ for C54 TiSi 2 used further for integration in the fabrication process. This was a sufficiently low resistance for our thermistor application. Additionally, it is recommended to realize the whole silicidation procedure in the shortest possible time frame. In particular, the first anneal after the Ti deposition and the etching of TiN.

In further sections the structural and electrical analysis of the $\mathrm{TiSi}_{2}$ thin films are discussed.

\subsubsection{Morphological characterization of the $\mathrm{TiSi}_{2} /$ poly-Si thin films}

Cross-sectional High Resolution Transmission Electron Microscopy (HRTEM) images were taken using a Philips CM300ST-FEG TEM system (Philips, Eindhoven, The Netherlands). All HRTEM images were recorded at an acceleration voltage of $300 \mathrm{kV}$ without an objective aperture. Two samples were analyzed following these fabrication steps:

- after the anneal at $700{ }^{\circ} \mathrm{C}$ and before strip of TiN;

- after the anneal at $900{ }^{\circ} \mathrm{C}$. 

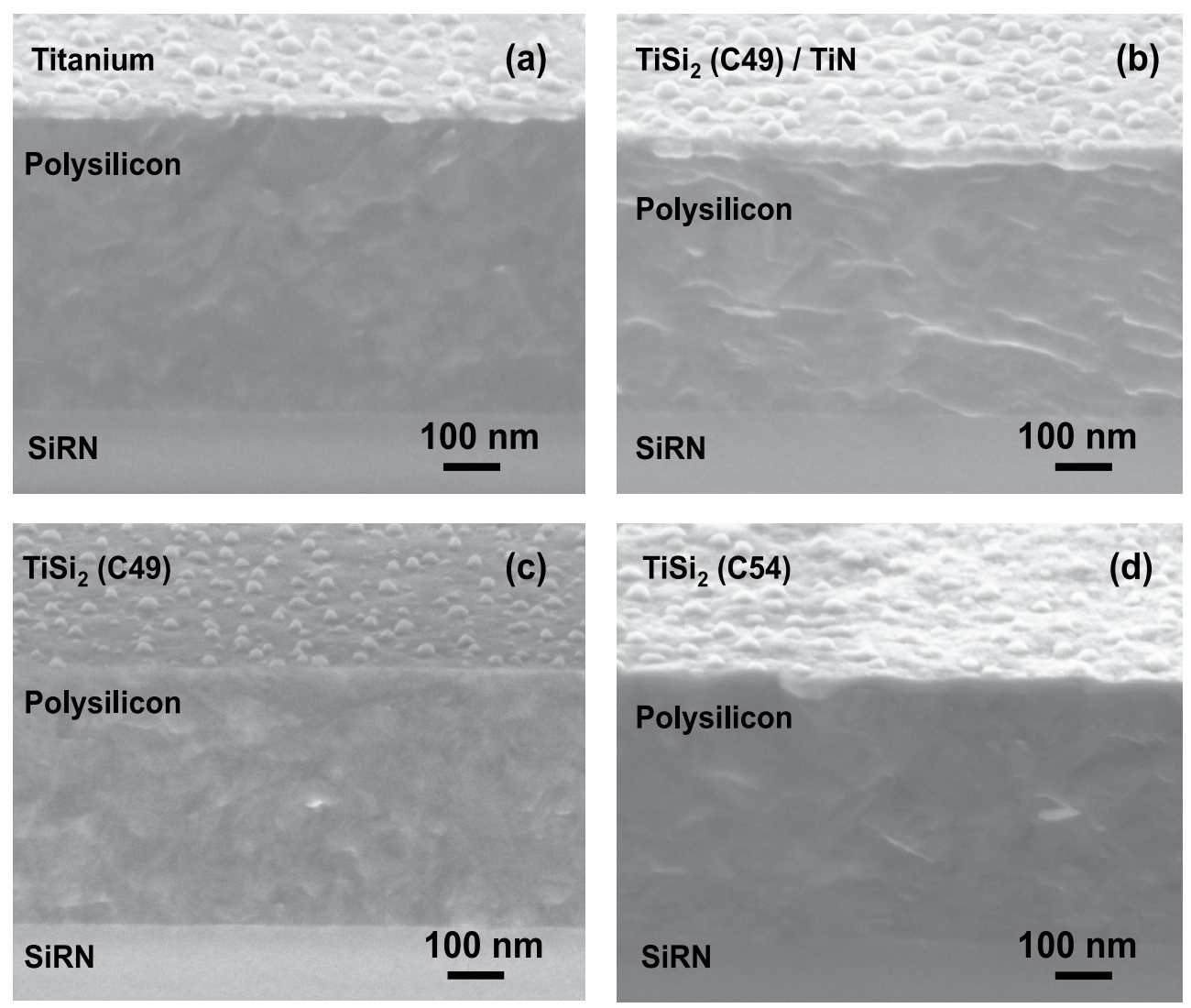

Figure 4.2: HRSEM cross-sectional view of the Si/SiRN/poly-Si/TiSi2 stack after deposition of Ti and before the first anneal (a), after the first anneal at $700^{\circ} \mathrm{C}$ before (b) and after (c) the etching of the TiN and unreacted Ti and after the second anneal at $900^{\circ} \mathrm{C}(d)$.

The aim of TEM analysis was to measure the thickness of the formed $\mathrm{TiSi}_{2}$ and TiN. Using Fast Fourier Transform (FFT) we were able to measure the spacings of the atomic planes. During the measurements internal calibration was performed for the (111) plane of poly-Si on both samples to identify an off-set correction factor. The off-set was estimated to be ca. $0.04 \AA$.

In order to determine the crystalline orientation of the $\mathrm{TiSi}_{2} \mathrm{X}$-Ray Diffractograms (XRD) were taken using a Philips XRD model Expert System (Panalytical, Almelo, The Netherlands). High-angle measurements were performed in the $2 \Theta$-range from $20^{\circ}$ to $60^{\circ}$. Four samples from the same wafer were analyzed:

(a) $\mathrm{Si} / \mathrm{SiRN} /$ poly-Si/Ti directly after the Ti sputtering and prior to any annealing;

(b) $\mathrm{Si} / \mathrm{SiRN} /$ poly-Si/TiSi (C49)/TiN after the $700{ }^{\circ} \mathrm{C}$ anneal and prior to the TiN etch;

(c) $\mathrm{Si} / \mathrm{SiRN} /$ poly-Si/TiSi (C49) after the $700{ }^{\circ} \mathrm{C}$ anneal and strip of the TiN;

(d) $\mathrm{Si} / \mathrm{SiRN} /$ poly-Si/TiSi (C54) after the $900{ }^{\circ} \mathrm{C}$ anneal.

X-ray photoelectron spectroscopy (XPS) surveys were performed on two TiSi 2 samples using a Quantera SXM scanning XPS microprobe (Physical Electronics, Inc., Chanhassen, MN, USA). The first sample was TiSi ${ }_{2}$ as formed. The second sample was from the same wafer subjected to deposition of $50 \mathrm{~nm}$ oxide by Plasma Enhanced Chemical Vapour Deposition (PECVD) at $300{ }^{\circ} \mathrm{C}$. The XPS analyses were performed to identify the content of $\mathrm{Ti}$ and $\mathrm{Si}$ in the $\mathrm{TiSi}_{2}$ layer, to verify the presence of any contamination (such as $\mathrm{O}$ and $\mathrm{N}$ ) and to study the influence of the PECVD $\mathrm{SiO}_{2}$ on $\mathrm{TiSi}_{2}$. Element spectra scans were taken during depth profiling. The composition of the $\mathrm{TiSi}_{2}$ layer was measured at a given point in depth of $10 \mathrm{~nm}$. The times are $3 \mathrm{~min}$ and $8 \mathrm{~min}$ $30 \mathrm{sec}$ for pure $\mathrm{TiSi}_{2}$ and $\mathrm{SiO}_{2}$-capped sample, respectively. 


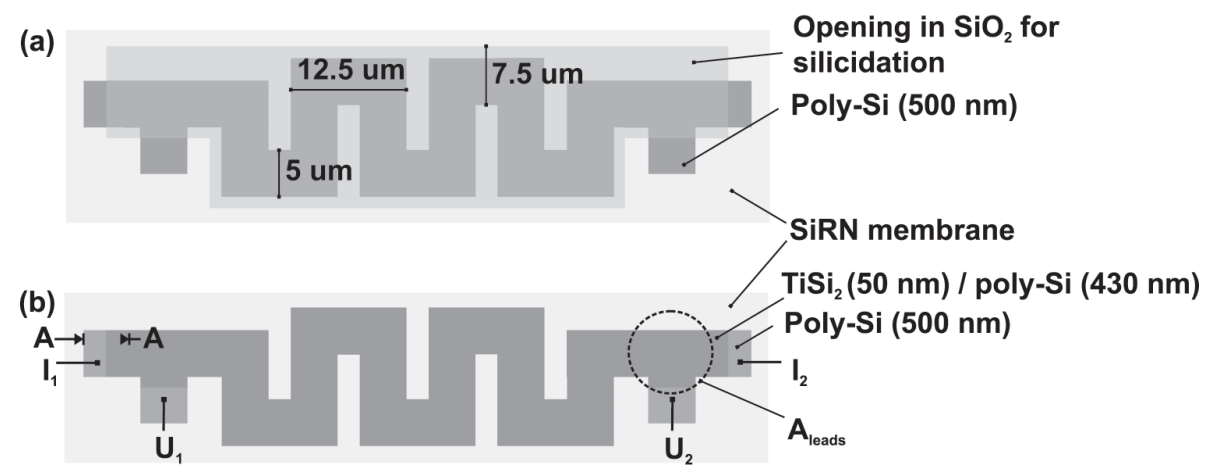

Figure 4.3: The $\mathrm{TiSi}_{2} /$ poly-Si resistor outlook showing dimensions of resistor, an opening in the $\mathrm{SiO}_{2}$ layer which was exposed to Ti (a) and an area of the poly-Si resistor converted to silicide (b).

Atomic Force Microscopy (AFM) in tapping-mode was used to measure the roughness and estimate surface morphology before and after the silicidation.

\subsubsection{Electrical characterization of the $\mathrm{TiSi}_{2} /$ poly-Si thin films}

The TCR and the stability of the poly-Si and TiSi 2 /poly-Si thin films were studied from $25^{\circ} \mathrm{C}$ to $800{ }^{\circ} \mathrm{C}$. The measurement setup was equipped with four probes at a fixed position $(16 \times 16 \mathrm{~mm})$. A heating element and an aluminum nitride substrate holder allowed temperature ramps up to $800{ }^{\circ} \mathrm{C}$ were used for in-situ electrical characterization of $\mathrm{TiSi}_{2} /$ poly-Si. The substrate was mounted with a silver thermal paste on the holder to ensure a good thermal contact. The temperature was regulated via a thermo-couple mounted under the sample. Measurements were performed in vacuum.

The measurements on the $\mathrm{Si}$ substrate were valid in the lower temperature range, i.e. from room temperature to $200-250{ }^{\circ} \mathrm{C}$. Above $250{ }^{\circ} \mathrm{C}$ the SiRN-film loses its isolating properties and may provide a short circuit to the intrinsic Si substrate. This effect was investigated by Tiggelaar et al. [105] The FS substrate was used for measurements up to $800{ }^{\circ} \mathrm{C}$.

The FS substrates were used to permit the high temperature resistance measurements on the thin films. The temperature was ramped between 50 and, subsequently, 200, 400, 600 and $800{ }^{\circ} \mathrm{C}$. After each annealing step sufficient time was given to stabilize the temperature at $50{ }^{\circ} \mathrm{C}$ again. This allowed us to extract the TCR and to test the presence of any electrical degradation (hysteresis). A stability test was performed on thin films that were already exposed to $800{ }^{\circ} \mathrm{C}$. During this measurement the temperature was ramped between 200 and 500 ${ }^{\circ} \mathrm{C}$ and stabilized after each heating ramp at $200{ }^{\circ} \mathrm{C}$. The wafer was kept for $5 \mathrm{~min}$ at $500{ }^{\circ} \mathrm{C}$ during each cycle. The resistance was monitored in-situ to observe the change in resistance.

\subsection{Fabrication of the temperature sensors and heaters}

\subsubsection{Design overview}

The design of the $\mathrm{TiSi}_{2}$ resistive structures used in this study is shown in Figure 4.3. A meander-shaped $\mathrm{TiSi}_{2} /$ poly-Si thermistor consisted of about $430 \mathrm{~nm}$ thick poly-Si and $50 \mathrm{~nm}$ thick $\mathrm{TiSi}_{2}$. The width $(5 \mu \mathrm{m})$ and length $(100 \mu \mathrm{m})$ were selected to form a resistor of about 100-150 $\Omega$. The resistor had poly-Si/Aluminum (Al) leads for an electrical readout in the 4-probe configuration to eliminate parasitic resistances during the measurements. The area where poly-Si leads were connected to the resistor was also subjected to the silicidation process. This eliminated possible failures in measurements associated with a high contact resistance or (and) a temperature gradient between the active part of resistor and the connections to the leads. 


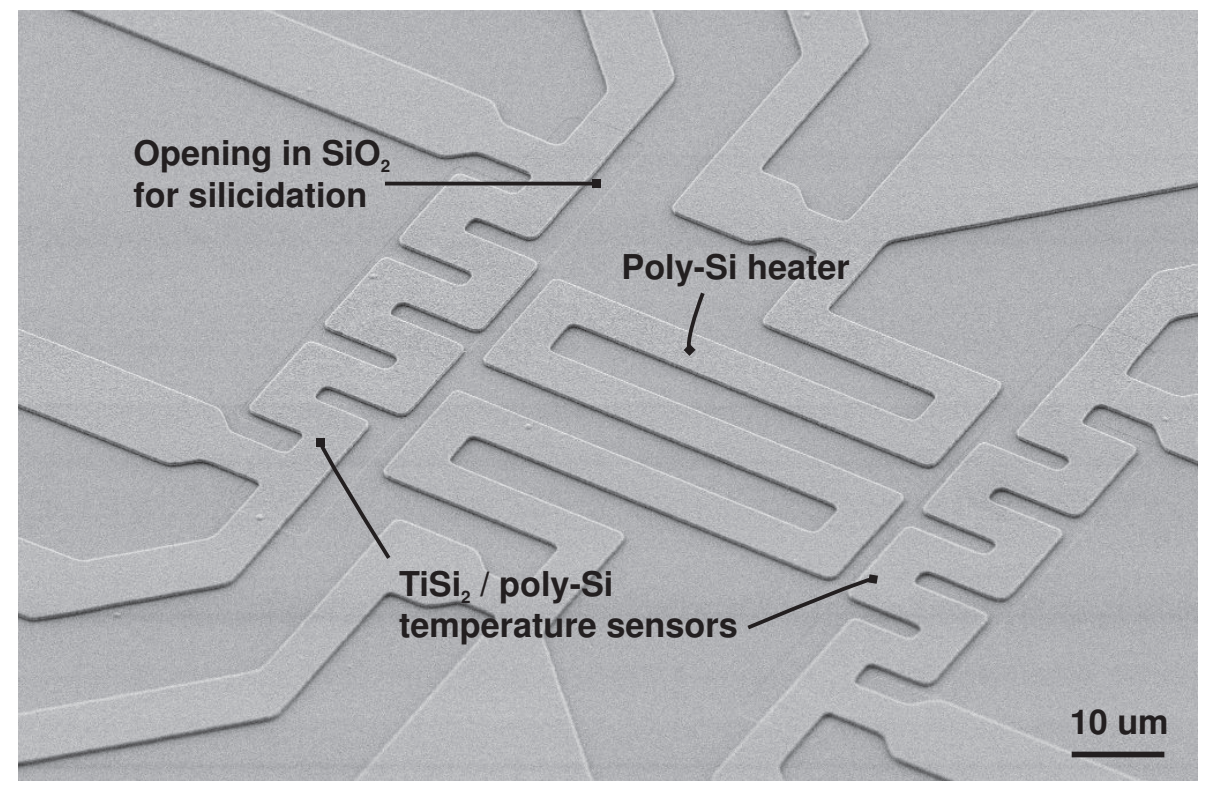

Figure 4.4: HRSEM top view image of the sensor device.

\subsubsection{Fabrication process flow}

The $11,4 \mathrm{k} \Omega$ poly-Si resistor used for heating was $5 \mu \mathrm{m}$ wide $230 \mu \mathrm{m}$ long. In these wide $\mathrm{TiSi}_{2} /$ poly-Si lines no dimensional limitation was expected. Dimensional limitation is characteristic for nanoscale designs, where the width of silicide line is comparable to the size of the silicide grains. An overview of the heater and temperature sensors is shown in Figure 4.4. The main steps of the fabrication procedure are schematically demonstrated in Figure 4.5. On a $\{100\}$ oriented Si wafer we deposited $200 \mathrm{~nm}$ of LPCVD SiRN and 500nm of LPCVD poly-Si. The resistance of the poly-Si was defined using ion implantation as discussed before. The resulted sheet resistance was ca. $190-220 \Omega /$ sq. The patterning of the poly-Si into resistive structures was achieved by RIE in $\mathrm{SF}_{6}, \mathrm{CHF}_{3}$ and $\mathrm{O}_{2}$ plasma with an Olin-17 photoresist mask. The structures were covered by a 50 $\mathrm{nm}$ PECVD $\mathrm{SiO}_{2}$ oxide. The thin oxide film served as an electrical isolator for the poly-Si heaters and as a protection to the poly-Si areas not used for the formation of the $\mathrm{TiSi}_{2}$. Before the silicide formation the openings in the oxide were defined by standard lithography with the Olin-17 photoresist (as shown in Figure 3 ). The 50 $\mathrm{nm}$ Ti film was deposited by DC magnetron sputtering at a base pressure of $2 \times 10^{-7} \mathrm{mbar}$. It is important to keep the background pressure below $1 \times 10^{-6}$ mbar to ensure oxygen-free metal deposition. This was followed by a $700{ }^{\circ} \mathrm{C}$ anneal step in forming gas and selective etching of the TiN. After the strip of TiN, a $900{ }^{\circ} \mathrm{C}$ anneal in forming gas was carried out. At this stage the poly-Si and $\mathrm{TiSi}_{2} /$ poly-Si resistive structures were realized. The $\mathrm{TiSi}_{2} /$ poly-Si temperature sensing structures were formed by the silicidation reaction between $\mathrm{Ti}$ and the patterned poly-Si. This is a self-aligned process, i.e. the silicidation will take place only on the poly-Si area which was in a direct contact with Ti. This manufacturing approach allowed formation of the highly resistive poly-Si heaters and the $\mathrm{TiSi}_{2} /$ poly-Si temperature sensors within one layer. The technological details of the silicidation process were discussed in section 3.1. Next, the structures were covered with another $50 \mathrm{~nm} \mathrm{PECVD} \mathrm{SiO} 2$. Standard lithography with the Olin-17 was used to define the openings in the $\mathrm{SiO}_{2}$ layer. The contact holes were etched using a buffered hydrofluoric acid (BHF) etching solution. A $400 \mathrm{~nm}$ of $\mathrm{Al}$ was sputtered and patterned with standard Al wet etchant (MERCK 115435.200). Next, the structures were annealed in $\mathrm{N}_{2}$ at $450{ }^{\circ} \mathrm{C}$ for $20 \mathrm{~min}$ to form a stable ohmic contacts to resistive structures. Thermal insulation was realized by bulk micromachining of $\mathrm{Si}$, i.e. anisotropic wet etching in $25 \%$ potassium hydroxide solution $(\mathrm{KOH})$ with SiRN as the etch-stop layer. During the Si etching, the front side of the wafer containing the functional structures was protected in a stainless steel holder. 


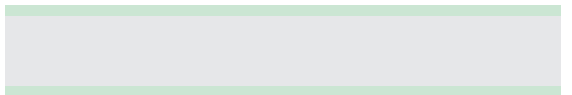

(i) Deposition of $200 \mathrm{~nm}$ LPCVD SiRN

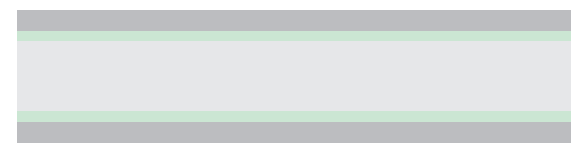

(ii) Deposition of $500 \mathrm{~nm}$ LPCVD poly-Si, ion implantation of B

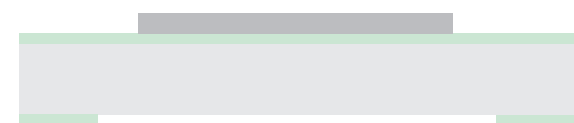

(iii) RIE patterning of the poly-Si, opening of the window for backside etching

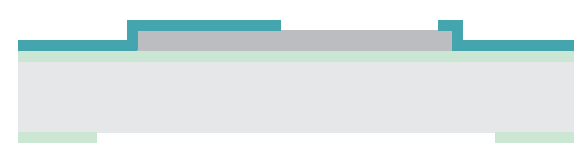

(iv) Deposition of PECVD $\mathrm{SiO}_{2}$, etching of window for silicidation in $\mathrm{SiO}_{2}$

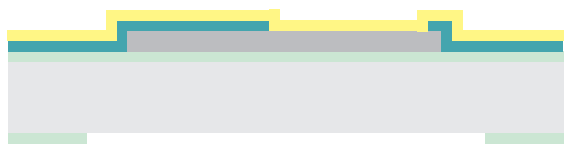

(v) DC magnetron sputtering of Ti

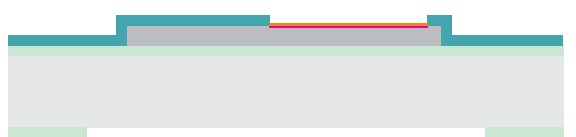

(vi) RTA anneals in forming gas, etching of $\mathrm{TiN}$, deposition of PECVD $\mathrm{SiO}_{2}$

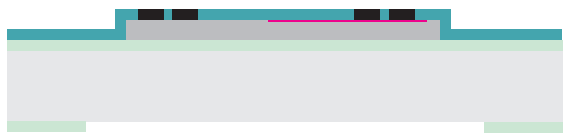

(vii) Formation of vias for metallization in BHF

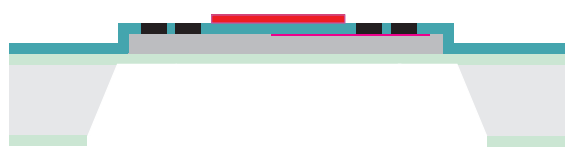

(x) Deposition of Al, etching of Si

Figure 4.5: The fabrication process flow for the suspended-membrane TiSi $2_{2} /$ poly-Si resistive temperature sensors. 

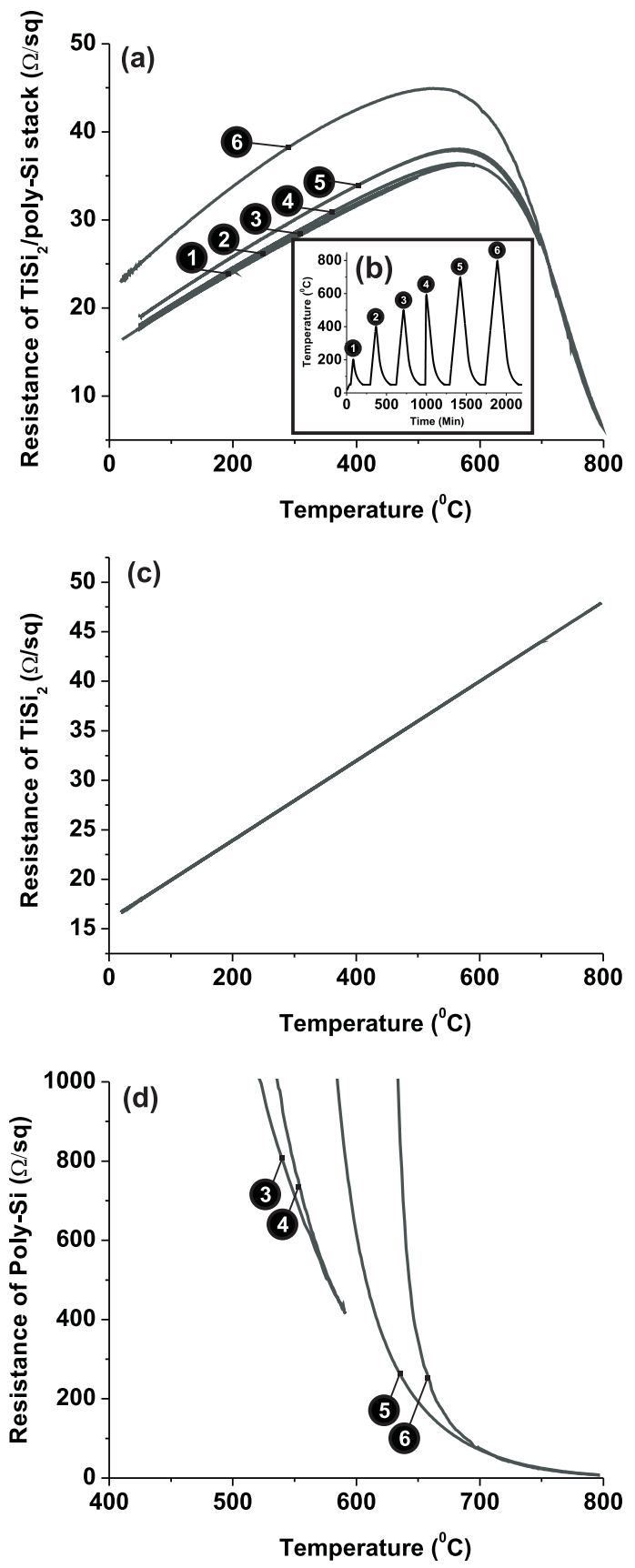

Figure 4.6: Contribution of poly-Si and TiSi $i_{2}$ to the total resistance of TiSi2/poly-Si stack on Si/SiRN substrate: (a) measured temperature dependence of TiSi $/$ /poly-Si stack under the temperature ramps applied in time (b); derived temperature dependence of resistance of TiSi $i_{2}$ layer (c), indicating linear TCR and no hysteresis; derived temperature dependence of resistance of poly-Si layer $(d)$, indicating contribution of poly-Si and intrinsic Si substrate at temperatures above $450^{\circ} \mathrm{C}$. 


\subsection{Results and discussion}

\subsubsection{Thermo-electrical properties of the $\mathrm{TiSi}_{2} / \mathrm{poly}-\mathrm{Si}$ thin films}

Figure 4.6 shows the dependence of the sheet resistance on temperature for the $\mathrm{TiSi}_{2} /$ poly-Si stack formed on the $\mathrm{Si} / \mathrm{SiRN}$ substrate. The contribution of the $\mathrm{TiSi}_{2}$ and the poly-Si sheet resistance to the total resistance of parallel $\mathrm{TiSi}_{2} /$ poly-Si stack is shown, where $R_{s, T i S i 2}$ is calculated from the measured values $R_{s, T i S i 2 / p o l y-S i}$ and $R_{s, p o l y-S i}$ according to Equation 4.1.

$$
\frac{1}{R_{s, T i S i 2 / \text { poly }-S i}}=\frac{1}{R_{s, p o l y-S i}}+\frac{1}{R_{s, T i S i 2}}
$$

Due to the conductive properties of the $\mathrm{SiRN}$ above $250{ }^{\circ} \mathrm{C}$, the intrinsic substrate contributed to the measured resistance (the negative TCR above $500{ }^{\circ} \mathrm{C}$ was due to the $\mathrm{Si}$ substrate). To avoid this effect, the thermoelectrical properties of the $\mathrm{TiSi}_{2} /$ poly-Si stack were also analyzed extensively on the FS substrates. In Figure
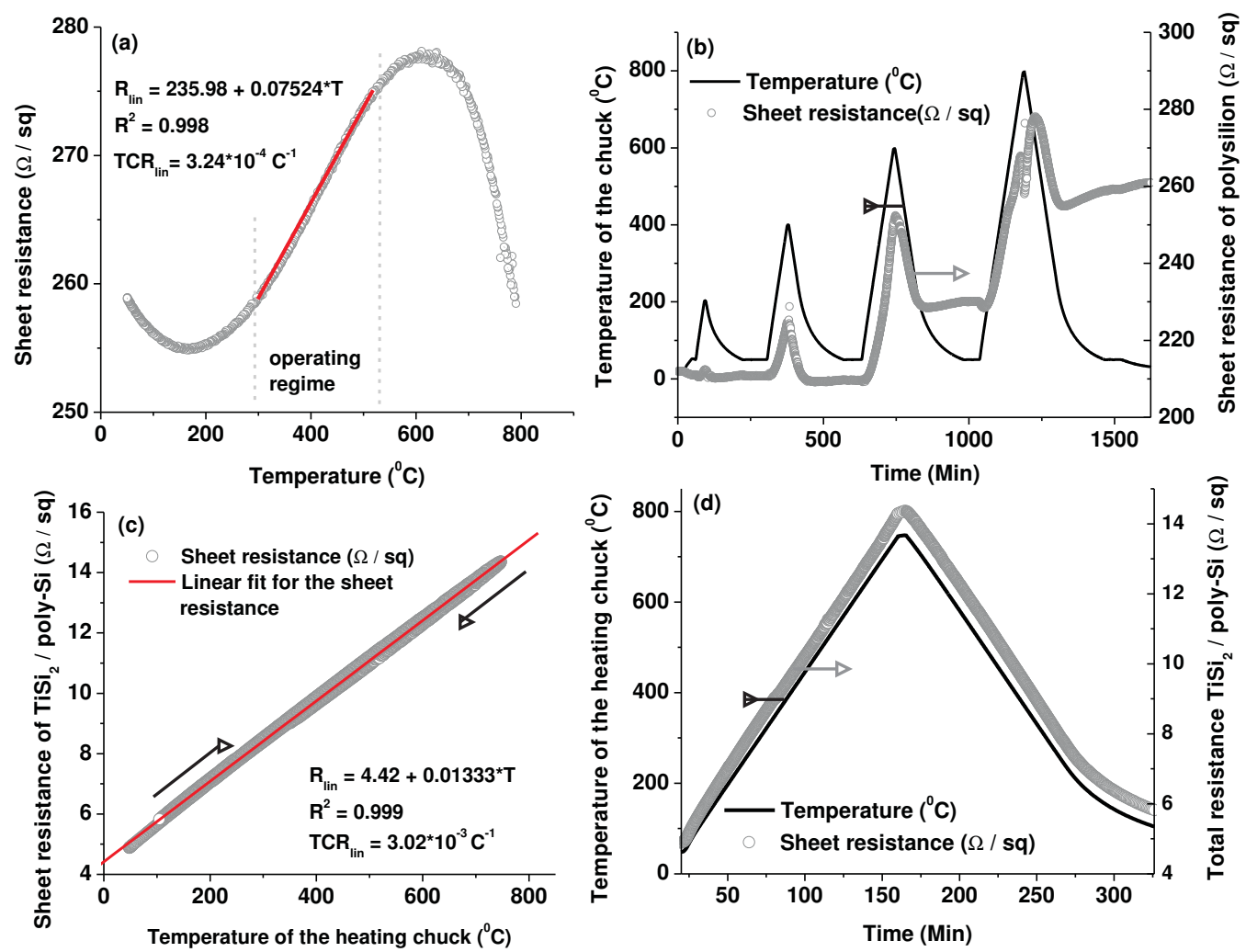

Figure 4.7: The temperature dependence of the poly-Si (a) and TiSi $2 /$ poly-Si (b) sheet resistance and the thermal stability of poly-Si $(c)$ and $\mathrm{TiSi}_{2} /$ poly-Si $(d)$ under applied temperature ramps. Figures $7 a$ and $7 b$ reprinted from [71] with permission from Elsevier.

4.7 we present the temperature dependence of resistance for a system of parallel resistances $\mathrm{TiSi}{ }_{2} / \mathrm{poly}-\mathrm{Si}$ on the FS as a function of temperature (c) and time (d). We found a linear temperature dependence of resistance. TCR values were between $(3.01-3.15) \times 10^{-3} /{ }^{\circ} \mathrm{C}$. No hysteresis was observed during the temperature ramps. A stability test was performed by several temperature cycles between $200{ }^{\circ} \mathrm{C}$ and $500{ }^{\circ} \mathrm{C}$. The test indicated no degradation of material due to thermal or electrical stress (see Figure 4.8). The results of poly-Si/B+ measured with the equal conditions on FS (see Figures 4-7a, 4-7b) show that poly-Si itself exhibited a much lower TCR (extrapolated for the linear part for about $2.5 \cdot 10^{-4} /{ }^{\circ} \mathrm{C}$ ) and its resistance depends non-linearly on temperature. In practice, the poly-Si based integrated thermistors have to be stabilized by high current densities or high 


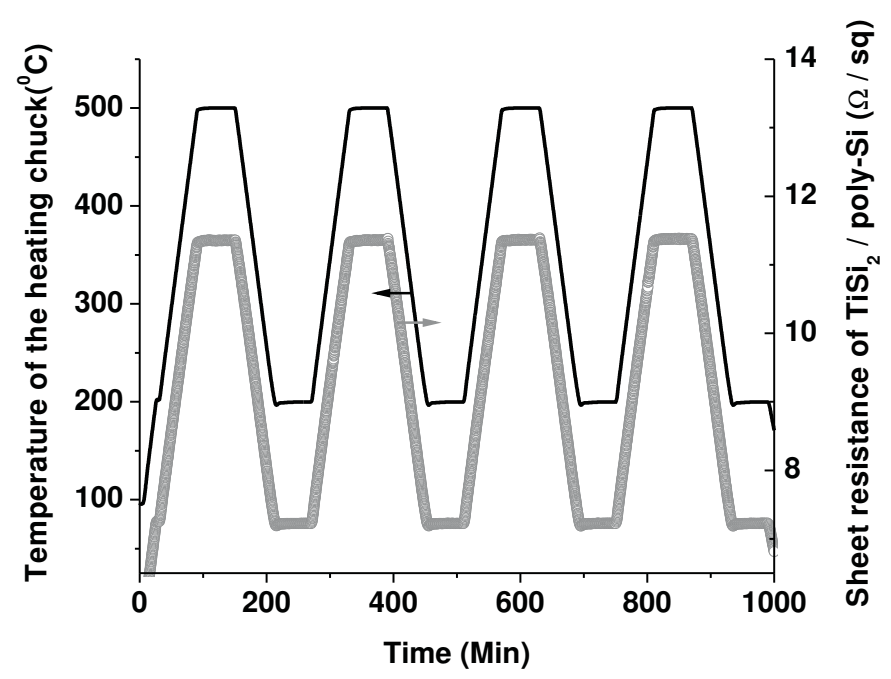

Figure 4.8: The thermo-electric stability test of TiSi2/poly-Si performed by ramping temperature between 200 ${ }^{\circ} \mathrm{C}$ and $500^{\circ} \mathrm{C}$.

temperature ramps. The addition of the $\mathrm{TiSi}_{2}$ clearly improved the thermo-electrical characteristics in this respect.

We tested the influence of the passivation layer on the resistive properties of poly-Si and subsequent formation of $\mathrm{TiSi}_{2}$. The goal of this test was to identify whether any redistribution of dopant or other processes occurred during the deposition of high temperature (TEOS) and low temperature (PECVD) oxides which may cause alteration of the resistance of the poly-Si or formed silicide. The $\mathrm{TiSi}_{2}$ layer was formed on three samples:

- Poly-Si without passivation;

- Poly-Si with $50 \mathrm{~nm}$ TEOS oxide deposited at $800^{\circ} \mathrm{C}$;

- Poly-Si with $50 \mathrm{~nm}$ PECVD oxide deposited at $300{ }^{\circ} \mathrm{C}$.

The TEOS, PECVD oxides were removed from poly-Si surface prior to deposition of Ti in BHF solution. In

Table 4.3: The sheet resistance of TiSi $/$ /poly-Si after the oxide deposition at $800{ }^{\circ} \mathrm{C}$ (TEOS) and $300{ }^{\circ} \mathrm{C}$ (PECVD) compared to poly-Si without the oxide layer.

\begin{tabular}{lcc}
\hline & \multicolumn{2}{c}{ Sheet resistance $(\Omega / \mathrm{sq})$} \\
\cline { 2 - 3 } & $1^{\text {st }}$ anneal & $2^{\text {nd }}$ anneal \\
\hline \hline no passivation & $16.31-17.4$ & $4.532-7.89$ \\
TEOS & $20.4-21.3$ & $7.16-7.43$ \\
PECVD & $17.54-20.94$ & $7.07-9$ \\
\hline
\end{tabular}

Table 4.3 a summary of the measured sheet resistances after first and second annealing steps is presented. The obtained resistances were in the same range for both annealing steps. Based on the results we conclude that the deviation in the resistance for the patterned structures was not caused by the passivation layer. 4-pointprobe measurement confirmed that $\mathrm{SiO}_{2}$ as the passivation layer did not alter resistive properties of poly-Si and, subsequently formed, poly-Si/TiSi ${ }_{2}$. In Figure 4.9a the SEM/FIB cross sectional image indicated a non-uniform silicide formation for the initial thickness of $50 \mathrm{~nm}$ Ti. This non-uniformity can be addressed to 

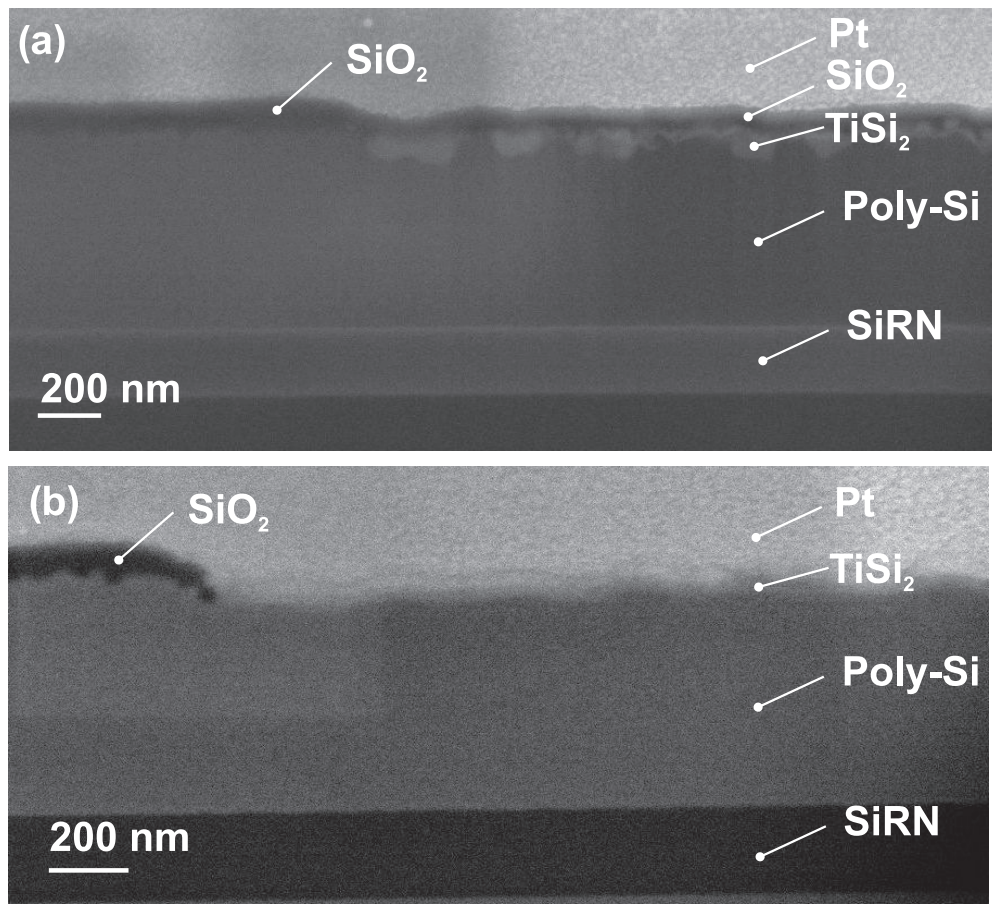

Figure 4.9: FIB/SEM cross-sectional images acquired at the edge of TiSi $2 /$ poly-Si resistor showing formation of the non-uniform TiSi $i_{2}$ thin film, improved thin film thickness uniformity as the Ti thickness was doubled (b).

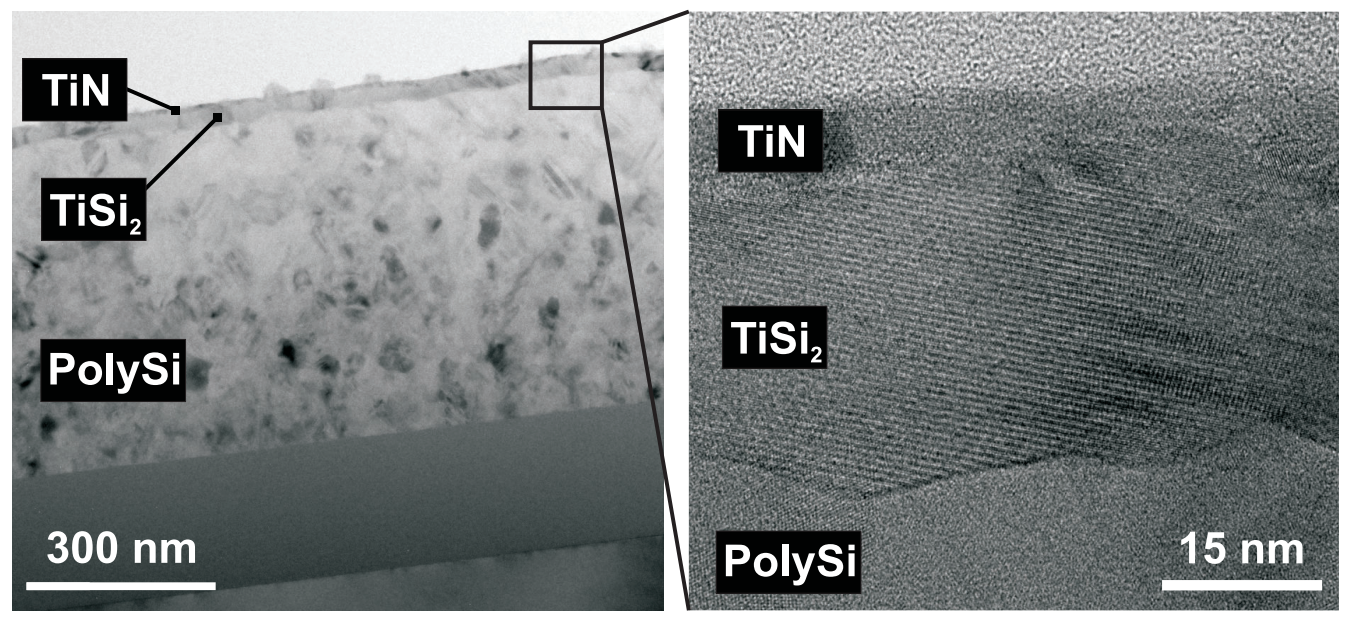

Figure 4.10: Cross-sectional TEM view on the substrate after first annealing at $700{ }^{\circ} \mathrm{C}$ showing the interface between the TiN, the C49 $\mathrm{TiSi}_{2}$ phase and the poly-Si. 


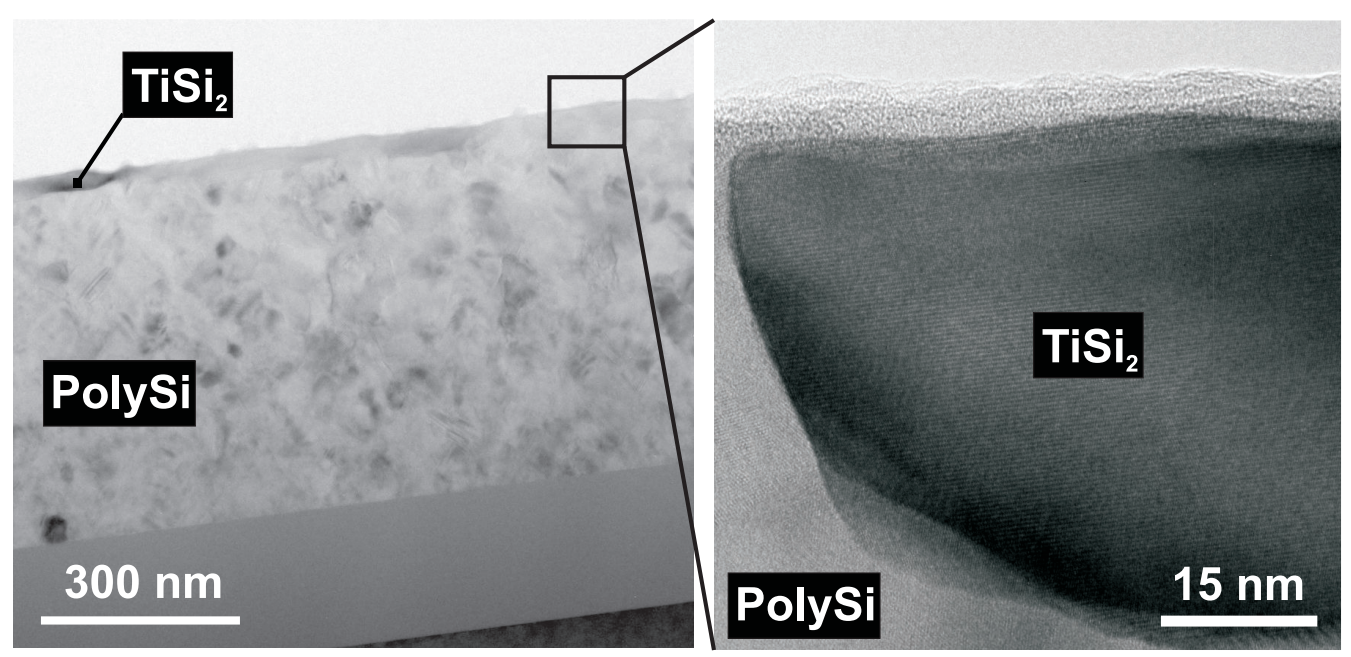

Figure 4.11: Cross-sectional TEM view on the substrate after second annealing at $900^{\circ} \mathrm{C}$ and stripping of the TiN and unreacted Ti, indicating the interface between the C54 phase and the poly-Si.

a presence of native oxide on the poly-Si, enhanced silicide formation at the grain boundaries or nucleation limited growth. For the $\mathrm{TiSi}_{2}$ /poly-Si resistors formed from $50 \mathrm{~nm} \mathrm{Ti}$ a much higher resistance than the desired value $(100-150 \Omega)$ was measured. To improve the uniformity and resistance a $100 \mathrm{~nm}$ thick Ti film was used. As a result an improved uniformity of the $\mathrm{TiSi}_{2}$ thin film (Figure 4-9b) and the desired resistance value was achieved.

\subsubsection{Morphological properties of the $\mathrm{TiSi}_{2} /$ poly-Si thin films}

TEM analysis of $\mathrm{TiSi}_{2}$ after $700{ }^{\circ} \mathrm{C}$ and $900{ }^{\circ} \mathrm{C}$ anneal revealed that both samples have well defined crystalline orientations. The TEM image of the sample after $700{ }^{\circ} \mathrm{C}$ (Figure 4.10) shown the formation of about $25 \mathrm{~nm}$ of $\mathrm{TiSi}_{2}$ and $5 \mathrm{~nm}$ of TiN. From in-situ FFT analysis a diffraction pattern was obtained and indicated a lattice spacing of $2.38 \pm 0.05 \AA$ for TiN. This spacing corresponded to the (111) orientation [137]. The lattice spacing of $2.17 \pm 0.05 \AA$ corresponded to the (121) orientation [138]. A larger lattice spacing of $6.66 \pm 0.05 \AA$ was also observed for the $\mathrm{TiSi}_{2}$ layer, but has not been identified. TEM images collected from the $\mathrm{TiSi}_{2}$ after 900 ${ }^{\circ} \mathrm{C}$ (Figure 4.11) shown an increase of the silicide thickness up to $50 \mathrm{~nm}$. From the FFT obtained from a diffraction pattern the experimental values for d-spacings were found: in the growth direction normal to the film surface $4.23 \pm 0.04 \AA$ and parallel to the film surface $2.29 \pm 0.03 \AA$. The obtained lattice spacings, though not corresponded to available database values, shown good agreement with the findings of Kittle et al. in [139]. Kittle discusses the effect of molybdenum impurities on the formation of titanium silicide, and reports lattice spacings of $2.27 \AA$ and $4.15-4.3 \AA$ for the C54 phase of silicide of unidentified orientation (the phase was not present in samples without Mo).

It should be mentioned that XRD had a limited applicability in in this study due to the fact that $\mathrm{TiSi}_{2}$ thin films that were investigated are between 25 and $60 \mathrm{~nm}$ thick and data available in the literature are based on powder samples. However, comparison to diffractograms obtained in other studies was still relevant. In Figure 4.12 $\mathrm{X}$-ray diffractograms taken on every step of the $\mathrm{TiSi}_{2}$ formation are presented. All four samples shown peaks of (111), (220), (311) attributed to the poly-Si layer. In graph (a) the peak appearing at $38.87^{\circ}$ represented a thin film of Ti with orientation (002). After the first annealing the two peaks at $41^{\circ}$ and $51^{\circ}$ were attributed to the (131)[129] and (002)[139] orientation of the C49 $\mathrm{TiSi}_{2}$ phase, respectively. Etching of TiN did not result in phase changes as can be seen in the X-ray diffractograms (Figure 4-12b, 4-12c). After the second anneal a strong peak at $42.33^{\circ}$ was observed and attributed to the (022)[129] orientation of the C54 phase or the (004) orientation. Two samples were investigated by XPS (see section 3.2). The results are presented in Figure 4.13. The elemental scan during depth profiling of the sample without the $\mathrm{PECVD} \mathrm{SiO}_{2}$ indicated some carbon contamination at the surface and an unknown amount of nitrogen. In the $\mathrm{TiSi}_{2}$ film only $\mathrm{Ti}$ and $\mathrm{Si}$ were 


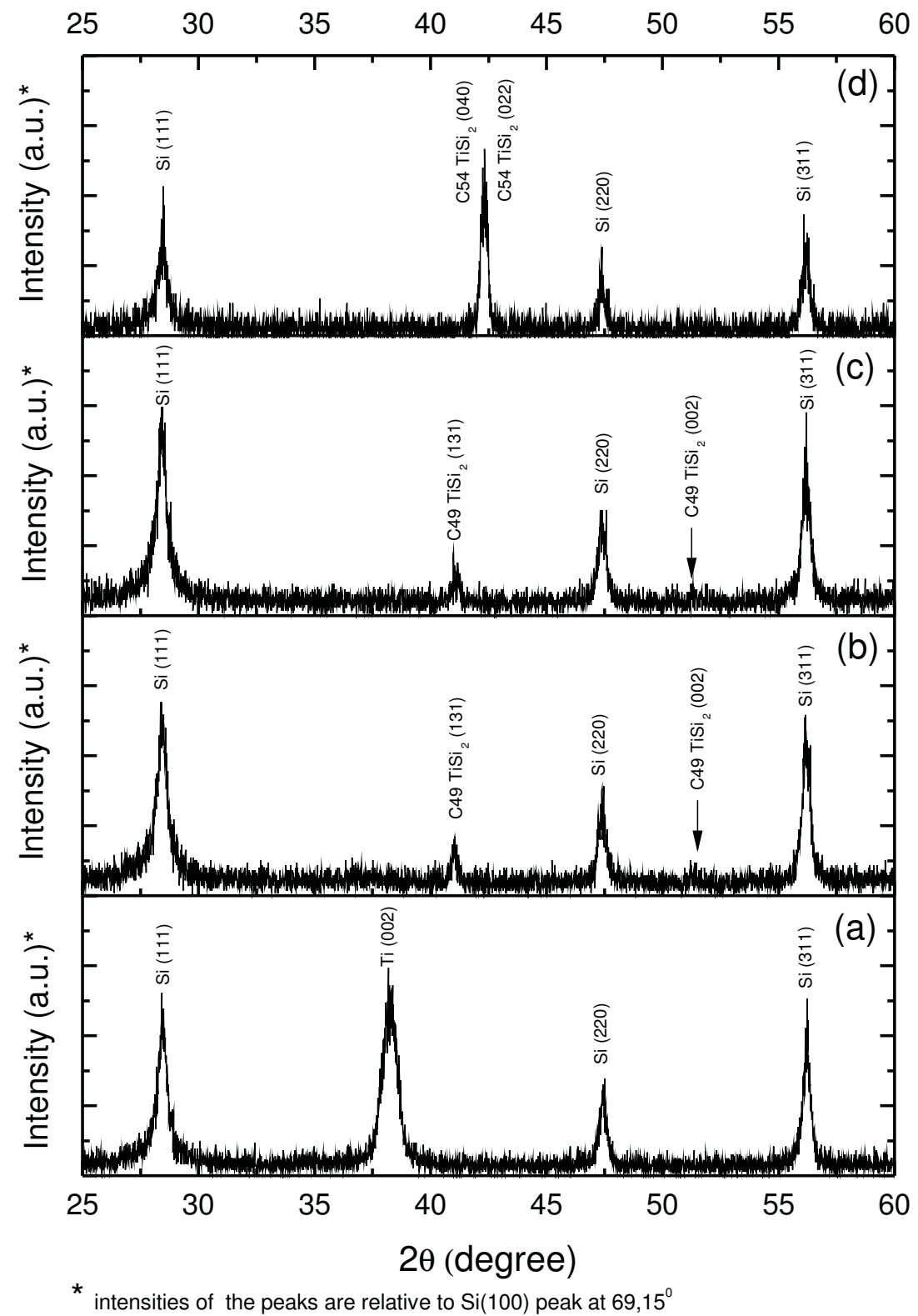

Figure 4.12: The X-ray diffractograms taken on each stage of the production of the TiSi2: (a) sputtering of Ti on the poly-Si, first anneal $700{ }^{\circ} \mathrm{C}$ before (b) and after (c) the TiN etch, (d) after the second anneal at $900{ }^{\circ} \mathrm{C}$. 

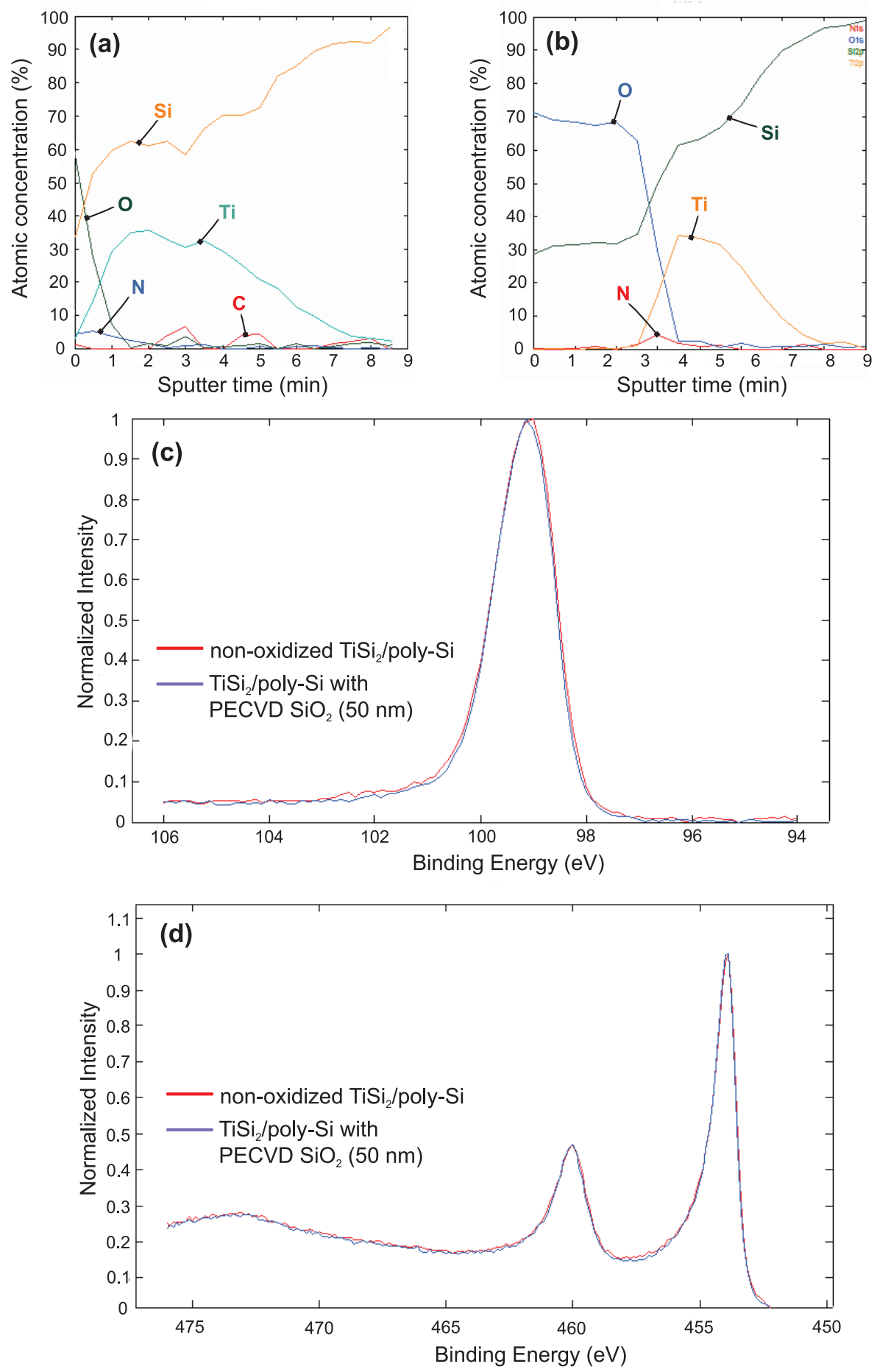

Figure 4.13: XPS analysis showing the atomic concentration of the elements as a function of sputter time for non-oxidized (a) and PECVD oxidized (b) TiSi 2 /poly-Si samples, and the binding energies for Si (c) and Ti (d). The relative peak height and the shape show that the binding energies of the Si, Ti atoms are similar. 

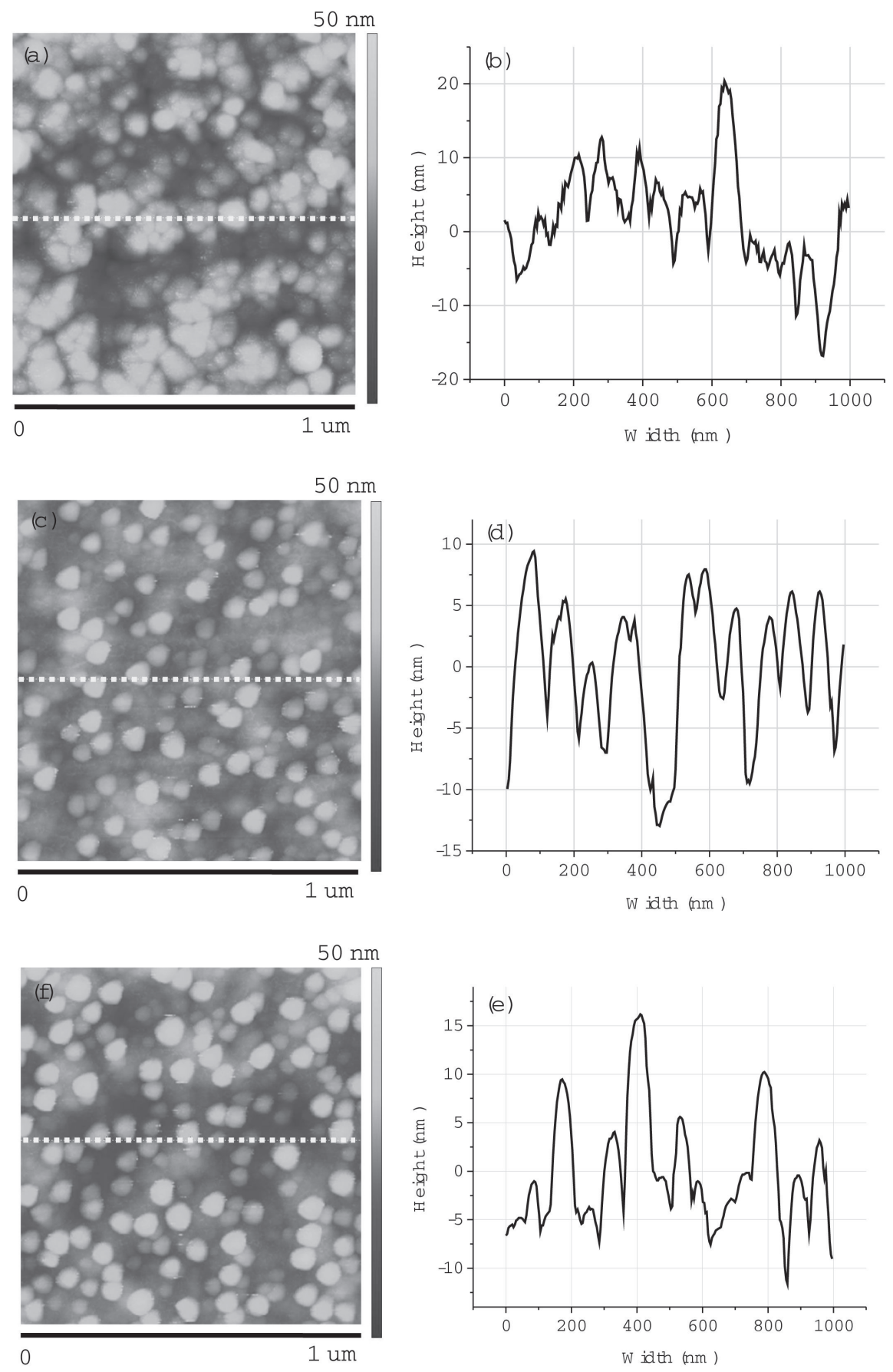

Figure 4.14: AFM images showing the morphology of the poly-Si thin film prior to silicidation (a, $b$ ), TiSi $2 /$ polySi thin films after the first anneal at $700{ }^{\circ} \mathrm{C}(c, d)$ and the second anneal at $900^{\circ} \mathrm{C}(f, e)$.

found. The interface of the $\mathrm{TiSi}_{2}$ and poly-Si found to be quite wide. This was caused by the roughness of the sample which was also revealed by AFM study. The surface of the oxidized sample shown almost no C contamination. The spectra for $\mathrm{Ti}$ and $\mathrm{Si}$ at the optimum depth for the two samples are almost identical. Thus, the bonds in the $\mathrm{TiSi}_{2}$ compound were identical. Nitrogen contamination was present in both samples. The concentration of nitrogen was higher in the oxidized sample. To conclude, the PECVD $\mathrm{SiO}_{2}$ layer did not alter structural properties of the formed $\mathrm{TiSi}_{2}$ layer and can be applied as a passivation. Using AFM the roughness of the produced silicide thin films was studied and compared to the initial poly-Si layer (see Figure 4.14). The formed $\mathrm{TiSi}_{2}$ layer shown a good homogeneity. A minor difference in morphology was observed between the 


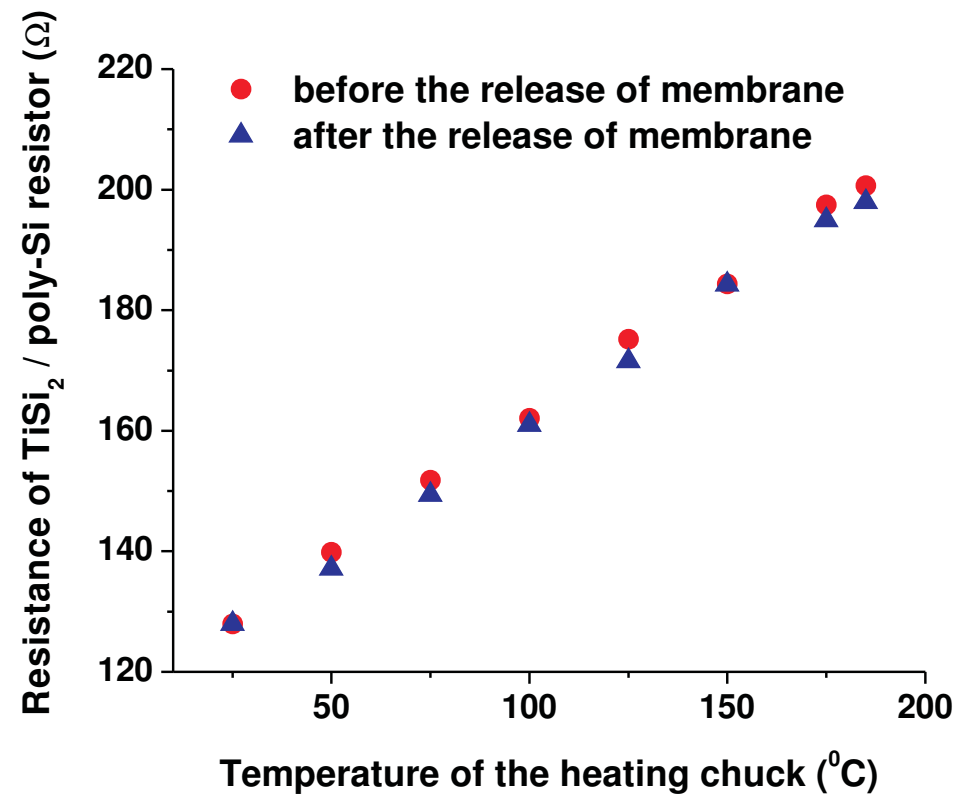

Figure 4.15: The TCR of a typical resistor derived for the range from $25^{\circ} \mathrm{C}$ to $185^{\circ} \mathrm{C}$ before and after the release of the membrane with the linear fit $R_{\text {lin }}=117+0.45 \times T$ and $T C R=3.85 \times 10^{-3} \rho C$ and $R_{\text {lin }}=115.87$ $+0.45 \times T$ and TCR $=3.883 \times 10^{-3} \rho C$, respectively. Voltages below $50 \mathrm{mV}$ were used for calibration and TCR measurements to prevent the self-heating effect.

samples representing $\mathrm{TiSi}_{2}$ surface after $700{ }^{\circ} \mathrm{C}$ and $900{ }^{\circ} \mathrm{C}$ anneals. Small, rough, hemispherical structures were observed. The diameter of these structures varied from $40 \mathrm{~nm}$ to $50 \mathrm{~nm}$.

\subsubsection{The TCR of the $\mathrm{TiSi}_{2} /$ poly-Si resistors}

The 4-probe electrical characterization of the $\mathrm{TiSi}_{2} /$ poly-Si resistors was done in the temperature range from $20{ }^{\circ} \mathrm{C}$ to $185^{\circ} \mathrm{C}$ using a Cascade Microtech Low Leakage Manual Probe Station (Cascade Microtech Inc., Beaverton, OR, USA) and a Keithley 4200 Semiconductor Characterization System (Keithley Instruments Inc., Cleveland, OH, USA). Dual current sweep measurements were performed from 0 to $100 \mu \mathrm{A}$. Resistive values were derived by averaging forward and back sweep values. The TCR of the TiSi 2 /poly-Si resistors was measured before and after the release of the membrane. The typical temperature behavior is presented in Figure 4.15. For different resistors the TCR was found to be in the range $(3.6-4) \times 10^{-3} /{ }^{\circ} \mathrm{C}$ and was not influenced by the release of the Si from the back side. For comparison, the TCR of Pt is $3.92 \times 10^{-3} /{ }^{\circ} \mathrm{C}$.

The TCR-value for the resistors was higher than observed previously for the flat, non-patterned TiSi $2 /$ poly-Si thin films. This is attributed to the different thickness of the $\mathrm{TiSi}_{2}(60 \mathrm{~nm}) /$ poly-Si $(430 \mathrm{~nm})$ resistor stack. It is worth to study the influence of the thickness of $\mathrm{TiSi}_{2}$ on TCR values.

\subsection{Conclusions}

Thin films of titanium silicide $\left(\mathrm{TiSi}_{2}\right.$ ) formed on heavily boron doped polycrystalline silicon (poly-Si/ $\mathrm{B}^{+}$) were applied for resistive temperature sensing. The TCR and the thermal stability were tested on $\mathrm{TiSi}_{2} / \mathrm{poly}-\mathrm{Si}$ thin films on $\mathrm{Si}$ and FS substrate and on $\mathrm{TiSi}_{2} /$ poly-Si resistors suspended on a SiRN membrane. The TCR for flat, non-patterned layers found to be $(2.9-3.1) \times 10^{-3} /{ }^{\circ} \mathrm{C}$ in the range of temperatures from 20 to $800{ }^{\circ} \mathrm{C}$. The TCR of suspended $\mathrm{TiSi}_{2} /$ poly-Si resistors was measured $(3.6-4) \times 10^{-3} /{ }^{\circ} \mathrm{C}$ from 20 to $185{ }^{\circ} \mathrm{C}$. 
To the best of our knowledge, this is the first time that the combination $\mathrm{TiSi}_{2} /$ poly-Si is used for temperature sensing purposes. In this contribution we provided the details for the design and realization of such $\mathrm{TiSi}_{2} / \mathrm{poly}$ Si temperature sensors. The fabrication process is suitable for integration into silicon-based lab-on-a-chip devices. 


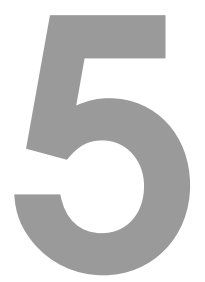

\section{Characterization of the $\mathrm{TiSi}_{2} /$ Poly-Si micro hotplates for microcalorimetric analysis of gaseous samples}

In this chapter the thermo-electrical characterization and analysis of the TiSi $2_{2} /$ poly-Si micro hotplate for microcalorimetric sensing applications is presented. Electrical, infrared surface imaging and transient thermal response measurements were performed on the devices.

The sensor consumes ca. $12 \mathrm{~mW}$ at an operational temperature of $350{ }^{\circ} \mathrm{C}$. Thermal imaging with an infrared camera indicated heating of the high-ohmic part with temperature gradient along the active sensor area estimated to be $4^{\circ} \mathrm{C} / \mu \mathrm{m}$ (at ca. $500^{\circ} \mathrm{C}$ ). The heating and cooling times were found to be ca. $1 \mathrm{~ms}$ and $8 \mathrm{~ms}$. The power vs temperature curve was determined while operating in both stationary and constant flow conditions, and shows a thermal resistance to the ambient of ca. $28^{\circ} \mathrm{C} / \mathrm{mW}$.

The detection principle in constant temperature mode based on model reactions of propane and hydrogen oxidation in air at temperatures $200-350^{\circ} \mathrm{C}$ and $100^{\circ} \mathrm{C}$, respectively, was demonstrated.

Due to the low signal-to-noise ratio (below 3 ), which is limited by the internal resistive noise, low resistance of the temperature sensors and the measurement method, it was not possible to differentiate between oxidation of propane, propyne and propene. We provide an outline of possible improvements for the current design. 


\subsection{Introduction}

MEMS-based micro hotplates [140]-[143] are useful for thermal sensing of gases [144], liquids [145] and solids [146] due to their minimal dimensions, low power consumption and, thus, easy integration.

In this study poly-Si-TiSi 2 -based micro hotplates are used as transducers for catalytic detection of combustible gases (hydrocarbons and hydrogen) in air, i.e. catalytic microsensors.

Power consumption, surface temperature distribution and thermo-electric properties of the materials comprising the micro hotplate are investigated. Based on this characterization and preliminary measurements with propane and hydrogen in air, conclusions are drawn on the feasibility of applying this design for the detection of combustible gases. Also, suggestions for design improvement are provided.

\subsection{Experimental}

\subsubsection{Design and fabrication}

The active area of the sensor consists of resistive structures suspended on a Silicon Rich Nitride (SiRN) membrane:

- A meander-shaped poly-Si (boron doped) heater of ca. $12 \mathrm{k} \Omega$, used to thermally actuate the reaction;

- $\mathrm{TiSi}_{2} /$ poly-Si temperature sensors of ca. $100 \Omega$, used for the control and measurement of temperature.

A top view of the sensor active area (a) and schematic cross section (b) are shown in Figure 5.1. Two sensor designs were implemented with the dimensions of the active area being $50 \times 50 \mu \mathrm{m}^{2}$ and $100 \times 100 \mu \mathrm{m}^{2}$, see Table 5.1. The dimensions of the active area determine the power consumption of the sensor and area covered with the catalyst (heat effect of the reaction is directly proportional to the amount of deposited catalyst). Considering low power requirements and given sufficient area to be covered with the catalyst, these two active areas were selected. The temperature sensors were located on both sides of the heater and parallel to the gas flow direction, and, thus during the operation were equally affected by thermal effects, such as flow and thermal properties of the ambient. The heater and temperature sensors were designed in a 4-probe configuration. The

Table 5.1: Dimensions of the sensor.

\begin{tabular}{lcc}
\hline Parameter & Design 1 & Design 2 \\
\hline \hline Suspended area $\left(\mu \mathrm{m}^{2}\right)$ & $400 \times 400$ & $800 \times 800$ \\
Active sensor area $\left(\mu \mathrm{m}^{2}\right)$ & $50 \times 50$ & $100 \times 100$ \\
Channel dimensions $\left(\mu \mathrm{m}^{3}\right)$ & $1000 \times 800 \times 200$ & $1000 \times 1000 \times 200$ \\
Catalyst patch $\left(\mu \mathrm{m}^{2}\right)$ & $50 \times 50$ & $100 \times 100$ \\
\hline
\end{tabular}

outer pair of leads was used to supply the heating current, while the inner pair was used to sense the voltage drop across the resistor. The leads used for supply of current were designed to be 10 times wider compared to the resistor line, for better heat confinement in the active heating area.

$500 \mathrm{~nm}$ Poly-Si, deposited by Low Pressure Chemical Vapor Deposition (LPCVD) implanted with boron $\left(\mathrm{B}^{+}\right.$) to concentrations of about $10^{20} \mathrm{at} / \mathrm{cm}^{-3}$, was used simultaneously as the heater material and basis for silicidation to form $\mathrm{TiSi}_{2}$ on the poly-Si/B+temperature sensors. The $\mathrm{TiSi}_{2}$ on poly-Si/B+ thin film was used for the control and measurement of temperature due to the high and stable TCR of $3.8 \times 10^{-3} /{ }^{\circ} \mathrm{C}$ up to $800{ }^{\circ} \mathrm{C}$. This temperature range is required to study thermally activated catalytic oxidation of hydrocarbons.

The silicidation procedure is discussed in detail in [72]. The resistance value for the $\mathrm{TiSi}_{2} / \mathrm{Poly} \mathrm{Si}$ temperature sensor with respect to thickness of the $\mathrm{TiSi}_{2}$ was investigated. Three fabrication runs were performed under the same sputtering conditions, with the thickness of the sputtered Ti thin films being 50, 100 and $150 \mathrm{~nm}$. The resistive values measured were compared and discussed further. 
Subsequently, a Plasma-Enhanced Chemical Vapor Deposited silicon oxide (PECVD SiO 2 ) film of $50 \mathrm{~nm}$ was deposited to passivate all resistors. $\mathrm{SiO}_{2}$ oxide might be not the best option in terms of diffusion barrier against $\mathrm{O}_{2}$. Despite that it was selected for passivation as it provides sufficient isolation, can be deposited at lower temperatures (at $300{ }^{\circ} \mathrm{C}$ ) and etches in BHF twice faster compared to LPCVD oxide. Oxide deposition was followed by BHF wet etching of openings to poly-Si and deposition of an aluminum (Al) metalization layer. The active sensor area contained a solid catalyst; a thin film of Pt on Ta with a thickness of $100 \mathrm{~nm} / 15 \mathrm{~nm}$ patterned using a metal lift-off method. The SiRN membrane was released in the last fabrication step to diminish the silicon mass being heated up and, thus reducing heat losses via conduction to the silicon substrate. For the same reason, the ratio of membrane size to the active area was kept 1:8. The assembly of the sensor is shown in Figure 5.1c. The silicon wafer was anodically-bonded to a glass wafer containing etched channels and blasted circular inlets for gas. The bonding ensured leakage-free device operation with flow rates at least up to $8 \mathrm{sccm}$. This was tested by applying hydrogen flows and measuring with the leak detector. At higher flow rates the 200 $\mathrm{nm}$ thin isolating membrane can fail. The volume of the channel (1.6-2 $\mu \mathrm{l})$ was selected to deliver a sufficient

(a)

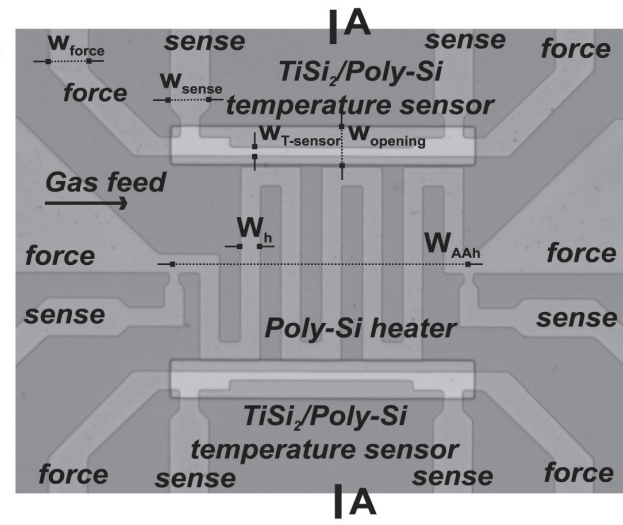

(b) A-A

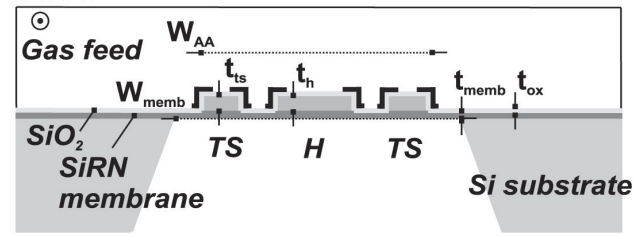

(c)

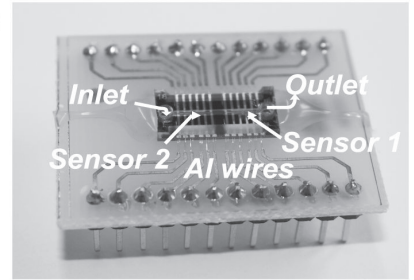

Figure 5.1: Top (a) and cross-sectional (b) views of the sensor and sensor assembly (c). The sensor 1 contains the catalyst film and sensor 2 does not. This configuration is used for blank test and quantification of gas flow and thermal conductance influence during the measurement.

amount of gas reactants to the sensor surface in a fast and controlled manner (the dimensions of the channel are shown in Table table 5.1). The PEEK fluidic connectors were glued with epoxy. The sensor was mounted on the custom-made printed circuit board and wire-bonded with $25 \mu \mathrm{m}$ thick Al/Si (99/1\%) wire. For more detailed description of the fabrication procedure see [72], [71].

\subsubsection{Catalytic films}

Two catalysts were tested: thin film Pt/Ta (100/15 nm) and Pt nanoparticles immobilized on porous alumina (5-10 wt \% Pt). The thin sputtered film was used previously in poly-si-based micro hotplates for detection of propane below $1 \%$ in air (see Chapter 3 of this thesis).

$\mathrm{Pt}$ on alumina sol is an interesting alternative to sputtered thin films as it provides high surface area (up to 400 $\mathrm{m}^{2} / \mathrm{g}$ as was measured) and more stable at high temperature oxidation reactions. Sol-gel-based catalyst was introduced for comparison with thin sputtered films and to increase sensitivity of the system (higher reaction 
Table 5.2: Electrical properties of the resistive materials.

\begin{tabular}{lcc}
\hline Parameter & Poly-Si (B) & $\mathrm{TiSi}_{2}(\mathrm{C} 54) / \mathrm{Poly}-\mathrm{Si}(\mathrm{B})$ \\
\hline \hline Sheet resistance $(\Omega / \mathrm{sq})$ & 220 & 6 \\
Average $\alpha\left(\mathrm{C}^{-1}\right)$ & $2.5 \times 10^{-4}$ & $(3.8) \times 10^{-3}$ \\
\hline
\end{tabular}

heats to be detected). Pt/alumina powder catalyst was prepared, dispersed in isopropanol and deposited dropwise on the gas sensor surface.

For more details on deposition and patterning of both types of catalysts refer to Chapter 7 of the thesis.

\subsubsection{Working principle}

The reactive gas (propane, propene, propyne, hydrogen) - air mixture is introduced into the channel. The active area of the sensor, containing a reactive catalyst, is heated up to the reaction temperature in a controlled manner. A catalyst is used to lower the operational temperature of the device and provide a means for selective detection. When the reactive mixture is brought in contact with the active area additional heat is released as a result of catalytic oxidation (exothermic) on the catalyst surface. This increases the average temperature across the active area of the membrane and, therefore, causes a change in resistance of the temperature sensors in accordance with the TCR. The sensor output measured - the decrease in power consumption of the heater, change in resistance of one of the temperature sensors - is proportional to the the heat released, which in its turn directly proportional to the concentration of the target gas. The sensor signal, depends on the ratio of concentrations propane to oxygen, activity of the catalytic thin film and reaction conditions (flow, temperature, concentration of detected gas). In addition, thermal conductive properties of the gas mixture can not be physically excluded from the measurement, but the influence is evaluated prior to the measurement.

\subsubsection{Electrical characterization}

A Süss MicroTec PM300 (SÜSS MicroTec AG, Garching, Germany) Manual Probe Station equipped with a Keithley 4200 (Keithley Instruments Inc., Cleveland, OH, USA) semiconductor characterization system was used for the DC I-V measurements.

The temperature coefficient of resistance (TCR) was determined from series of I-V data collected at elevated temperatures. A built-in, temperature-controlled heating chuck was used to collect I-V characteristics from room temperature to $200{ }^{\circ} \mathrm{C}$. A dual-current sweep was repeated over the range 0 to $100 \mu \mathrm{A}$. The average value of the electrical resistance was determined at $50 \mu \mathrm{A}$, which is sufficiently low to eliminate the influence of self heating. The TCR $(\alpha)$ was extracted from the linear fit parameters $\beta$ (slope) and $\mathrm{R}_{0}$ (resistance extrapolated to zero ${ }^{\circ} \mathrm{C}$ ) of the $\mathrm{R}(\mathrm{T})$ curve:

$$
R=R_{0}\left(1+\frac{\beta}{R_{0}} \cdot T\right)=R_{0}(1+\alpha \cdot T)
$$

The sheet resistance and the Temperature Coefficient of Resistance (TCR) of heater and temperature sensor material were calibrated prior to the measurements and are summarized in Table 5.2. Based on the I-V curves collected from the heater and the TCR of the $\mathrm{TiSi}_{2} / \mathrm{Poly}-\mathrm{Si}$ temperature sensor, the Power-Temperature curve (PT-curve) was derived in stationary conditions. A series of heater power vs temperature curves measured by the $\mathrm{TiSi}_{2} / \mathrm{Poly}-\mathrm{Si}$ temperature sensor were collected for various flow and ambient conditions.

\section{Stabilization of the poly-Si heater}

The material of the heater, poly-Si, has a tendency to drift. This drift can be caused by the effect of grains and grain boundary rearrangement. The highest temperature to which material was exposed during the fabrication is $800{ }^{\circ} \mathrm{C}$, which is still not sufficient to stabilize the resistive properties. For stabilization purposes the poly-Si 
heater was exposed to a DC current sweep ranging from 0 to $2.6 \mathrm{~mA}(18-22 \mathrm{~mW})$. The current was selected to be higher than the supply current (did not exceed $1.5 \mathrm{~mA}$ ) used during the sensor operation.

\subsubsection{Infrared surface imaging}

Thermal imaging of the sensor surface was used to estimate the temperature distribution across the heater area and membrane and to verify the effect of localized heating. IR imaging was performed using a XenICs infra-red (IR) camera (Xenics nv, Leuven, Belgium) equipped with InGaAs detector and operating in the range 0.9-1.7 $\mu \mathrm{m}$. A constant current bias was applied to the heater in the range from 0.7 to $1.5 \mathrm{~mA}$ using a Karl Süss PM8 Low Leakage Manual Probe Station and Keithley 4200 during the acquisition of IR images. The temperature of the surface was calculated based on the TCR (Section 2.2.1).

\subsubsection{Transient thermal response}

(a)
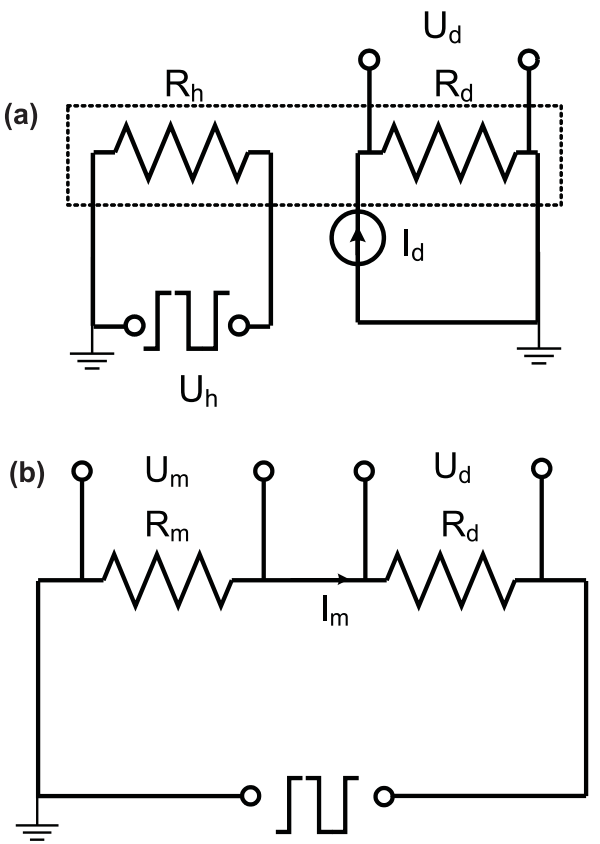

Figure 5.2: The measurement circuits used to determine the thermal time constant of the TiSi2/Poly-Si temperature sensors.

The transient thermal behavior of the micro hotplate was investigated in the range $100-250{ }^{\circ} \mathrm{C}$ which corresponded to a power dissipated by the poly-Si heater in the range $2.4-5.9 \mathrm{~mW}$. The thermal time constant $(\eta)$ of the $\mathrm{TiSi}_{2} /$ poly-Si temperature sensor was measured using two methods (see Figure 5.2):

1. As a thermal response to a stepped change in voltage applied to the poly-Si heater located closely on the same membrane, shown in Figure 5.2a;

2. As a thermal response to a stepped change in voltage applied to the $\mathrm{TiSi}_{2} /$ poly-Si temperature sensor directly, shown in Figure 5.2b;

An Agilent 33120A (Agilent, Santa Clara, CA, USA) function and arbitrary waveform generator was used to apply a square voltage pulse. A power amplifier was used to amplify the voltage signal. A digital oscilloscopeTextronix TDS 210 (Tektronix, Inc., OR, USA) was used for recording of the applied voltage pulses, voltage across the measured resistor and voltage across the tested resistor.

$\eta_{\text {rise }}$ was calculated as $63.2 \%$ of the difference between the initial temperature of the resistor and that of when 
exposed to a voltage step. $\eta_{\text {fall }}$ was calculated as the decay from the value when exposed to a voltage step to the initial value.

\subsubsection{Experimental setup}

The experimental setup used is shown in Figure 5.3. Agilent 34970A data acquisition unit, a Keithley 2410
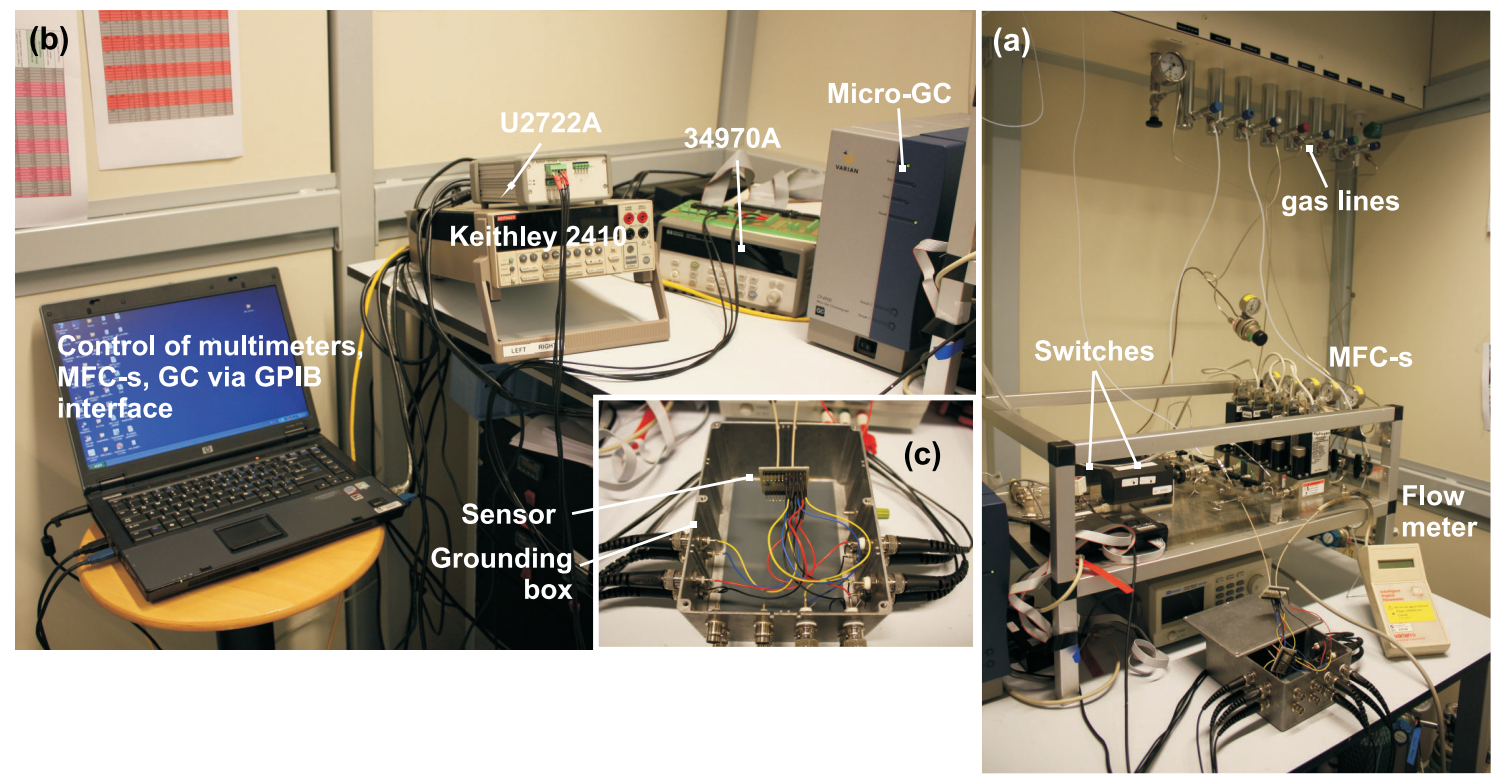

Figure 5.3: Experimental setup: (a) gas feed (b) instrumental source/measure units, Micro-GC and (c) grounding box with all electrical connections coming to the tested sensor.

source/measure unit were used for acquisition of the electrical parameters and Agilent U2722A source unit for precise supply of power. The constant temperature operational mode was used in the experiment. A PID heater-temperature sensor control loop and acquisition of electrical parameters in time was implemented in Lab View 8.6.1 (National Instruments Netherlands BV, Woerden, The Netherlands). The connection of the sensor device to instrumental source-measure units is indicated in Figure 5.4.

The supplied power to the poly-Si heater was controlled through accurate current sourcing and measurement of voltage drop across the heater. The temperature was determined from the change in resistance of $\mathrm{TiSi}_{2} / \mathrm{poly}_{-} \mathrm{Si}$ resistors in accordance with the TCR. The current used to measure the resistance was minimized to reduce the self-heating effect. The temperature of the membrane was kept constant during the measurement via the feedback control loop.

For the gas feed (connections are indicated in Figure 5.5) precision mass flow controllers from Brooks Instruments (Ede, The Netherlands) were used with the range 0-3 sccm for line 3, 0-10 sccm for lines 1,2,4 and 0-20 sccm for line 5. Two VICI 4-way micro electric actuators were used to switch between nitrogen (line 2) and a propane/nitrogen (line 3). By diluting the standard mixture with an additional nitrogen from the line 1, pulses of reactive gas at various concentrations were injected. The concentration of propane, propene, propyne in nitrogen was $1 \mathrm{vol} \%$, given a total flow of $3 \mathrm{sccm}$ and the flow range of the MFC-s reliable dilution was possible down to $100 \mathrm{ppm}$, which was tested by Micro-GC. Synthetic air was fed from the line 4. Nitrogen was used as a balance gas to keep the flow rate constant and minimize the influence of thermal conductivity. Hydrogen from line 5 was used for measurement as well as for reduction of the catalyst.

Before introducing the reactive mixture, the sensor was stabilized at the required temperature in nitrogen ambient till satisfactory stability of the set point temperature was reached (acceptable error is within +/- 0.05-0.1\% of the set point). For tests the total flow was varied between 1 and $3 \mathrm{sccm}$. The GC was operated in a continuous mode. The concentration of reactive gas in nitrogen was varied between $0.01-0.8$ vol \%. Instrumental air (purity 4.8) and nitrogen (purity 5.0) were fed continuously to the inlet line. The reactive gas/nitrogen mixture 


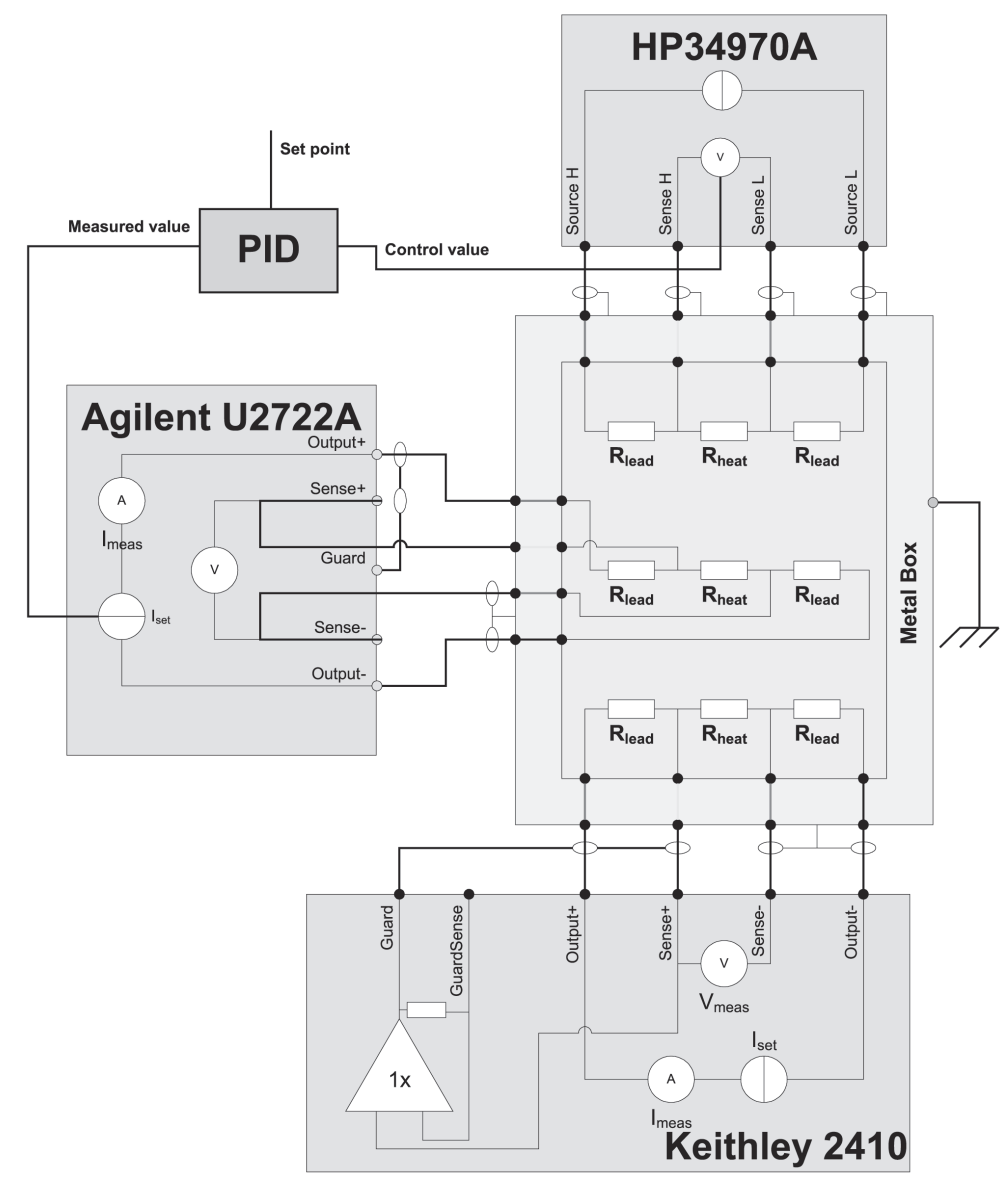

Figure 5.4: Schematic of the circuit used for the readout of all electrical parameters and constant temperature measurement.

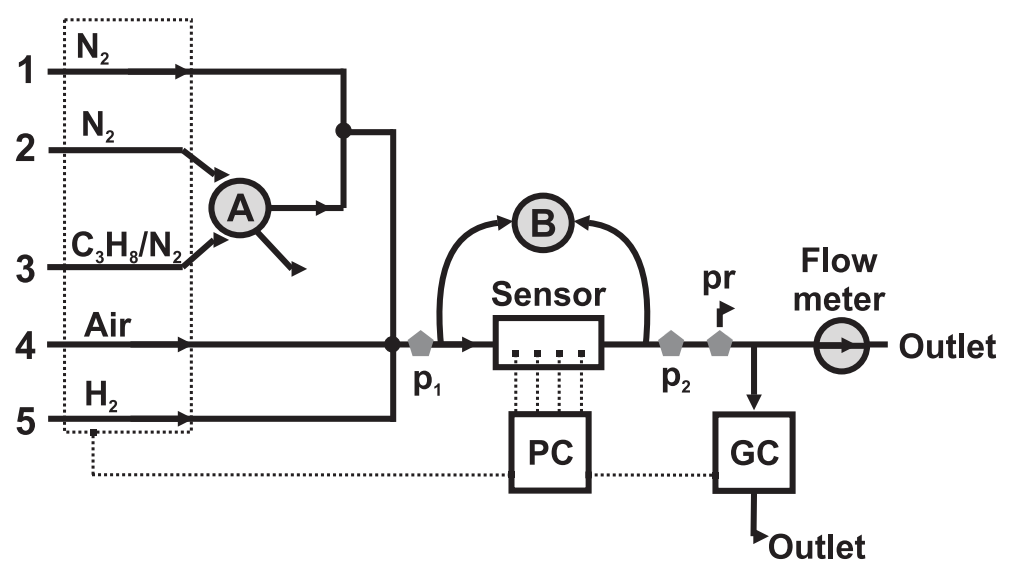

Figure 5.5: Schematic of the test setup: lines 1-5 mass flow controllers (MFC) for gas feed, pressure sensor $\left(p_{1}, p_{2}\right)$, gas chromatograph $(G C)$, microelectric actuators $(A, B)$, pressure reducer $(p r)$ and flow meter.

was diluted with additional nitrogen to the desired concentration, afterwards added to air. The amount of air is in stoichiometric proportion with the amount of reactive gases. In addition, we performed the experiments with conditions where air is higher than stoichiometric amount.

The CP-4900 micro gas chromatograph (Micro-GC) from Varian BV (Middelburg, The Netherlands) equipped with Molsieve and Propak Q columns was used to measure the concentrations of the gas components. Calibration was performed for propane, propyne, propene prior to the measurements in the range $0.8-0.1 \mathrm{vol} \%$. The 
Micro-GC was operated in continuous mode. Connection of the Micro-GC to the outlet of the chip was optimized in such a way that the reaction conditions on the chip (temperature, pressure) at the moment of sampling remain maximally equal to those without sampling. In practice, pressure drop and a concentration gradient along the line is difficult to eliminate completely. The sampling procedure was improved by incorporating a remote start. This allowed to sample to some extent independently from time delays introduced by the computer and to synchronize the time between the start of the reaction and start of the analysis using the Micro-GC.

Time and duration of the gas pulses were controlled by custom-made software. At first a baseline resistance corresponding to a nitrogen-air flow was measured. Then the pulses of propane/nitrogen were introduced into the sensor channel. The reacting gas (propane, propene, propyne) reacts with oxygen from air on the catalytic surface of the chip.

This results in generation of additional heat. This effect is accordingly balanced by decrease of power level to control the temperature constantly on the surface of the active area. Based on the resistive measurements the heat release of the reaction was identified and compared with the conversion data from CP-4900 micro gas chromatograph (Micro-GC).

\subsection{Results and Discussion}

\subsubsection{Electrical characterization}

\section{Temperature coefficient of resistance (TCR)}

The TCR was measured and found to be stable and positive within the range from room temperature to $200{ }^{\circ} \mathrm{C}$ (see Figure 5.6). Linear extrapolation was applied in the high temperature range, which is valid and based on previous findings [71]. The spread in TCR values between the measured series was found ca. $8 \%$ of the average TCR value, which is $3.5 \times 10^{-3} /{ }^{\circ} \mathrm{C}$. The TCR is comparable to commonly used thin metal temperature sensors, such as platinum $\left(3.7 \times 10^{-3} /{ }^{\circ} \mathrm{C}\right)$, nickel $\left(5 \times 10^{-3} /{ }^{\circ} \mathrm{C}\right.$, bulk value $)$ but the technology offers the advantages of compatibility with Si based processing and does not require sophisticated passivation solutions as in case of thin metal films to be used at high temperatures.

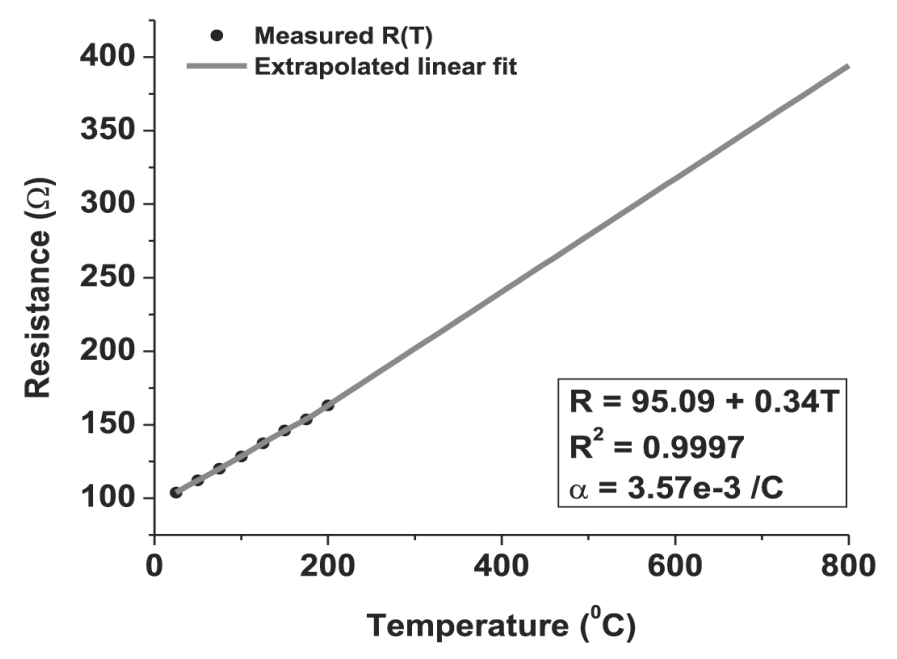

Figure 5.6: Temperature dependence of the resistance for the TiSi $2 /$ Poly-Si temperature sensors: extracted $\alpha=3.57 \times 10^{-3} \rho$ P. Same dependence was observed in case of all three resistor designs. 


\section{Resistance of temperature sensors}

The resistance of the poly-Si/TiSi 2 resistors is dependent on the initial thickness of the deposited Ti thin film (see Figure 5.7). The highest resistance was obtained for the run with $50 \mathrm{~nm}$ sputtered Ti thin films. Approximately half of this resistance was obtained with a thickness of sputtered thin film of $100 \mathrm{~nm}$. The third run, with $150 \mathrm{~nm}$ thick layers, deviates from this tendency and did not indicate even lower resistive values. This can be attributed to a non-clean contact or a change in the kinetics formation of the silicide. By means of variation in titanium thickness the sheet resistance and, thus, the nominal value of the final resistive structures can be controlled. The non-uniformity in the sputtered titanium layer also plays an important role. Due to nonuniform deposition of titanium (thicker at the center of the wafer) the resistive values increase by $15 \%$ towards the edges of the wafer.

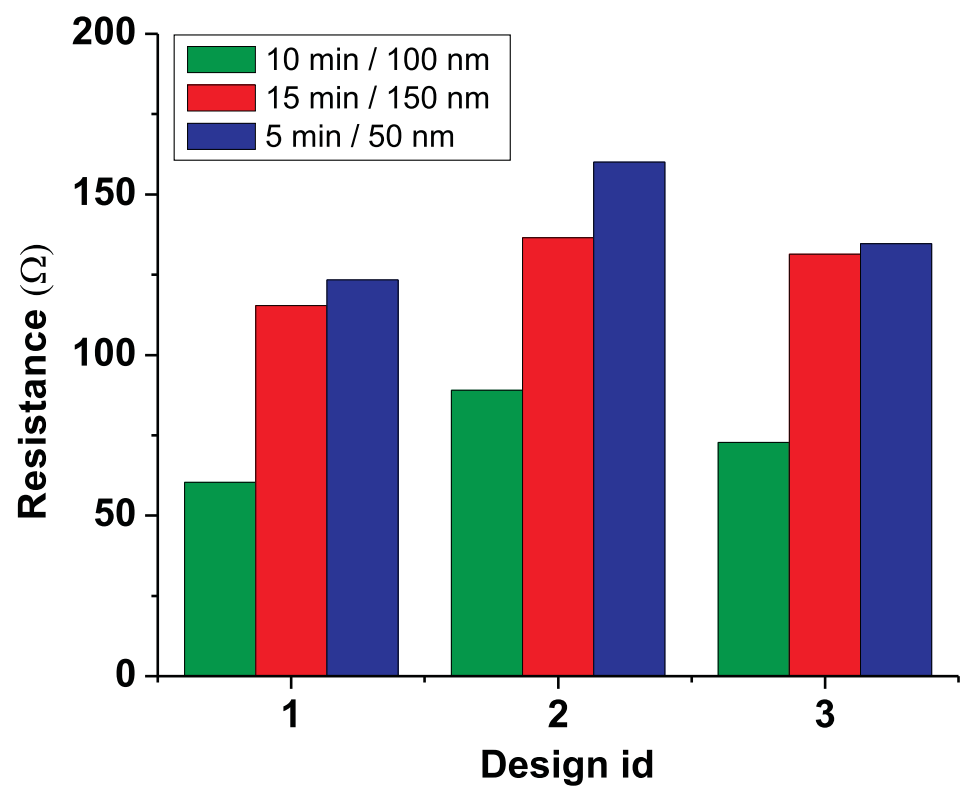

Figure 5.7: Measured resistance for three different resistor designs for three runs with thickness of Ti 50, 100, $150 \mathrm{~nm}$. The color depicts the fabrication run.

\section{Stabilization of the poly-Si heater}

All resistors exhibit a slight change in value with time. For applications where stability is critical, devices are commonly burned-in for at least one week. The range of full operational hours before stabilization may range from few to thousands.

Due to time limitations, as an alternative to conventional burn-in procedures a high current was passed through the poly-Si heater until stabilization in the resistance value was achieved (see Figure 5.8).

\section{Power-Temperature (PT) curve}

To characterize the thermal properties of the micro hotplate, the heater power vs temperature (PT-curve) of the active area of the sensor was measured and is presented in Figure 5.9. The temperature was determined from the TCR calibration of the $\mathrm{TiSi}_{2} /$ poly-Si resistive temperature sensor. The temperature of the active area reaches ca. $350{ }^{\circ} \mathrm{C}$ at the power level input of ca. $13 \mathrm{~mW}$. From the temperature vs power curve the thermal resistance of the suspended micro hotplate was found to be $28.85{ }^{\circ} \mathrm{C} / \mathrm{mW}$. The obtained thermal resistance 

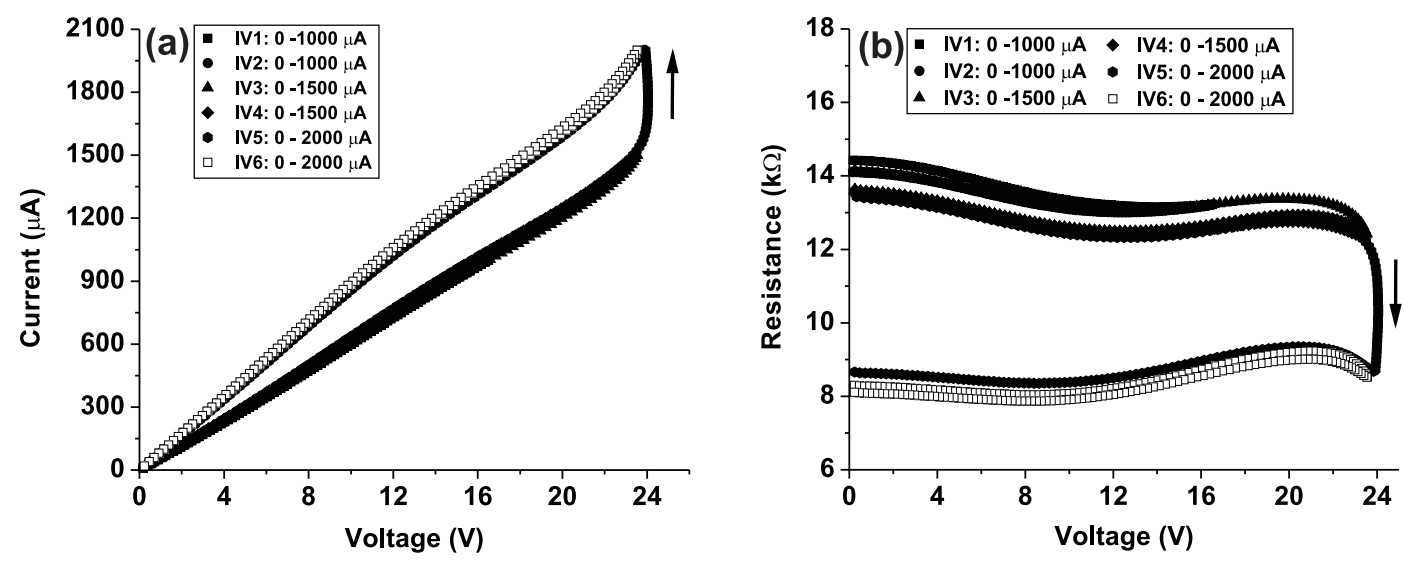

Figure 5.8: The current and resistance as a function of the applied voltage: "burn - in" procedure for stabilization of the poly-Si heater.
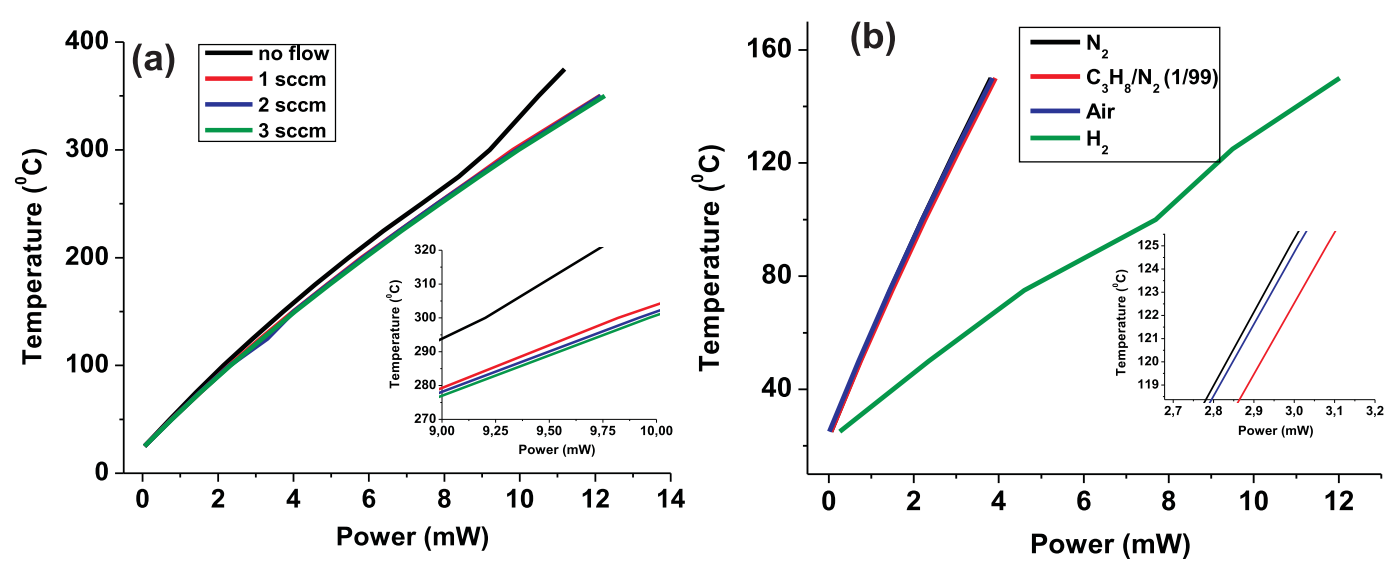

Figure 5.9: $P$-T curve collected at stationary conditions and in the presence of $N_{2}$ flow (a), and P-T curve collected in presence of $\mathrm{N}_{2}(25.83 \mathrm{~mW} / \mathrm{mK}), \mathrm{C}_{3} \mathrm{H}_{8} / \mathrm{N}_{2}$ (1/99 vol. \%, for $\left.\mathrm{C}_{3} \mathrm{H}_{8} 15.198 \mathrm{~mW} / \mathrm{mK}\right)$, air $(24 \mathrm{~mW} / \mathrm{mK})$ and $\mathrm{H}_{2}(180.5 \mathrm{~mW} / \mathrm{mK})$ of $2 \mathrm{sccm}(b)$.

is higher or comparable to current state-of-the-art micro gas sensors and sensor arrays [142]. From the PTcurve in stationary and constant flow of $\mathrm{N}_{2}$ conditions it can be seen that flow causes cooling of the active area of ca. $40{ }^{\circ} \mathrm{C}$ at $12 \mathrm{~mW}$ input power which corresponds to temperatures above $300{ }^{\circ} \mathrm{C}$. The effect is more pronounced when exceeding $250{ }^{\circ} \mathrm{C}$. The surface temperature was relatively constant for $1-3 \mathrm{sccm}$, thus the flow does not affect much the measurements. The total flow in this studied was kept at $3 \mathrm{sccm}$. The PT-curves collected for various gas ambients indicate that the presence of different ambients significantly influences the sensor power output due to differences in thermal conductivities of gases. The difference for nitrogen, air and propane/nitrogen is, however, minimal. The gases with drastically different thermal conductive properties, such as hydrogen, result in larger power output change.

\subsubsection{Infrared surface imaging}

The IR images of the membranes, power supplied to the heater and estimated temperatures are summarized in Figure 5.10. In order to convert IR intensity given in a.d.u. and collected as a function of the supplied power 
level to the temperature, the known $\mathrm{R}(\mathrm{T})$ and P-T curves, shown in Figure 5.6 and 5.9 respectively, were used and correlated with IR intensity. Clear IR images were obtained above $300{ }^{\circ} \mathrm{C}$. On some images the hot spot on the right side is observed. This is an artifact of the measurement system and not attributed to the device behavior. Imaging below $300{ }^{\circ} \mathrm{C}$ was limited by the sensitivity of the detector. From the images can be seen that the heat is localized on the high ohmic part, thus the leads remain "cold" compared to the heated area at least until $450-480{ }^{\circ} \mathrm{C}$ as estimated. Based on the relation between IR emission (expressed in arbitrary digital units) of the collected spectra and the temperature, an estimation of the temperature gradient along the active area of the heater can be made. However, it should be taken into account that the heater emits IR radiation in all directions, so the emission spectra from different sections of the heater may overlap. The temperature gradient was found to be ca. $4{ }^{\circ} \mathrm{C} / \mu \mathrm{m}$ at ca. $500{ }^{\circ} \mathrm{C}$ surface temperature. This is sufficiently uniform for carrying out catalytic studies.

(a)

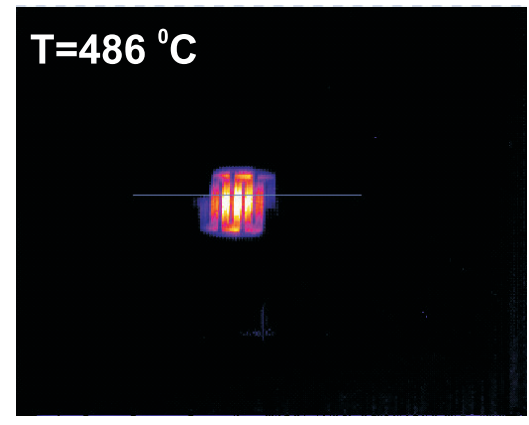

(b)

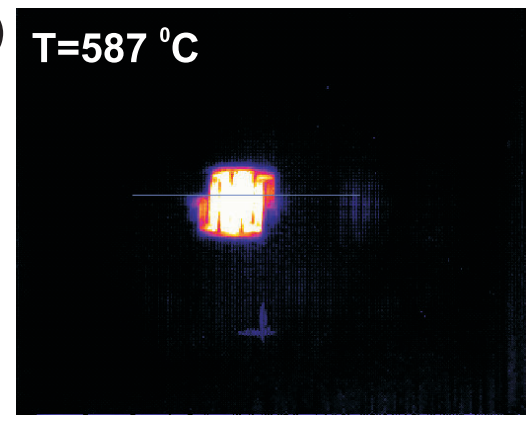

(c)

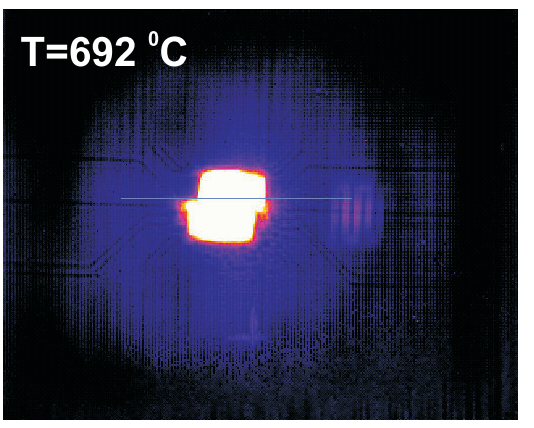

(d)

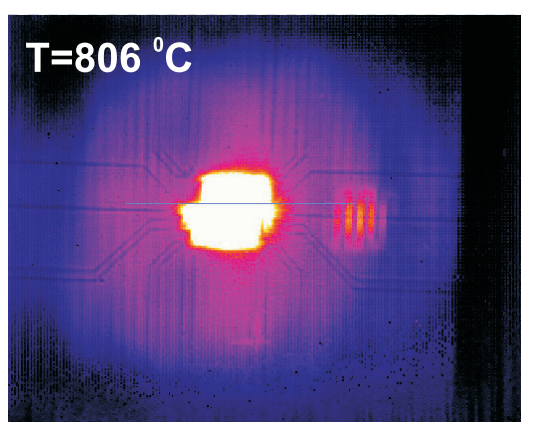

Figure 5.10: Infrared images of the sensor surface (design 2) taken at $15.8 \mathrm{~mW}(\mathrm{a}), 19.1 \mathrm{~mW}(\mathrm{~b}), 22.5 \mathrm{~mW}(\mathrm{c})$ and $26.2 \mathrm{~mW}(d)$.

\subsubsection{Transient thermal response}

The thermal heating and cooling constants of the TiSi $2 /$ poly-Si temperature sensor were determined by two methods as described in the Section 2.5. Measurements with both methods gave almost identical results. The heating and cooling times were found to be within $1 \mathrm{~ms}$ and $8 \mathrm{~ms}$, respectively, when voltage pulses were applied to the poly-Si resistor located on the same membrane (see Figure 5.11). For the measurement circuit indicated in Figure $5.2 \mathrm{~b}$ the heating time was found within $0.5 \mathrm{~ms}$. In the latter arrangement, the measured time constant is dominated by the $\mathrm{TiSi}_{2} /$ poly-Si resistor, while for the first measurement arrangement the time constant of the membrane is included. The short timescale within which the micro hotplate reaches thermal equilibrium with the environment allows analysis of various types of reactions. Slower heating and cooling profiles can be programmed and controlled via a PID control loop.

\subsubsection{Detection of reaction heats}

The reactions considered in the experiments are listed in Table 5.3 with corresponding enthalpies and ignition temperatures. According to our initial plan the detection of propane, propyne and propene was a target appli- 

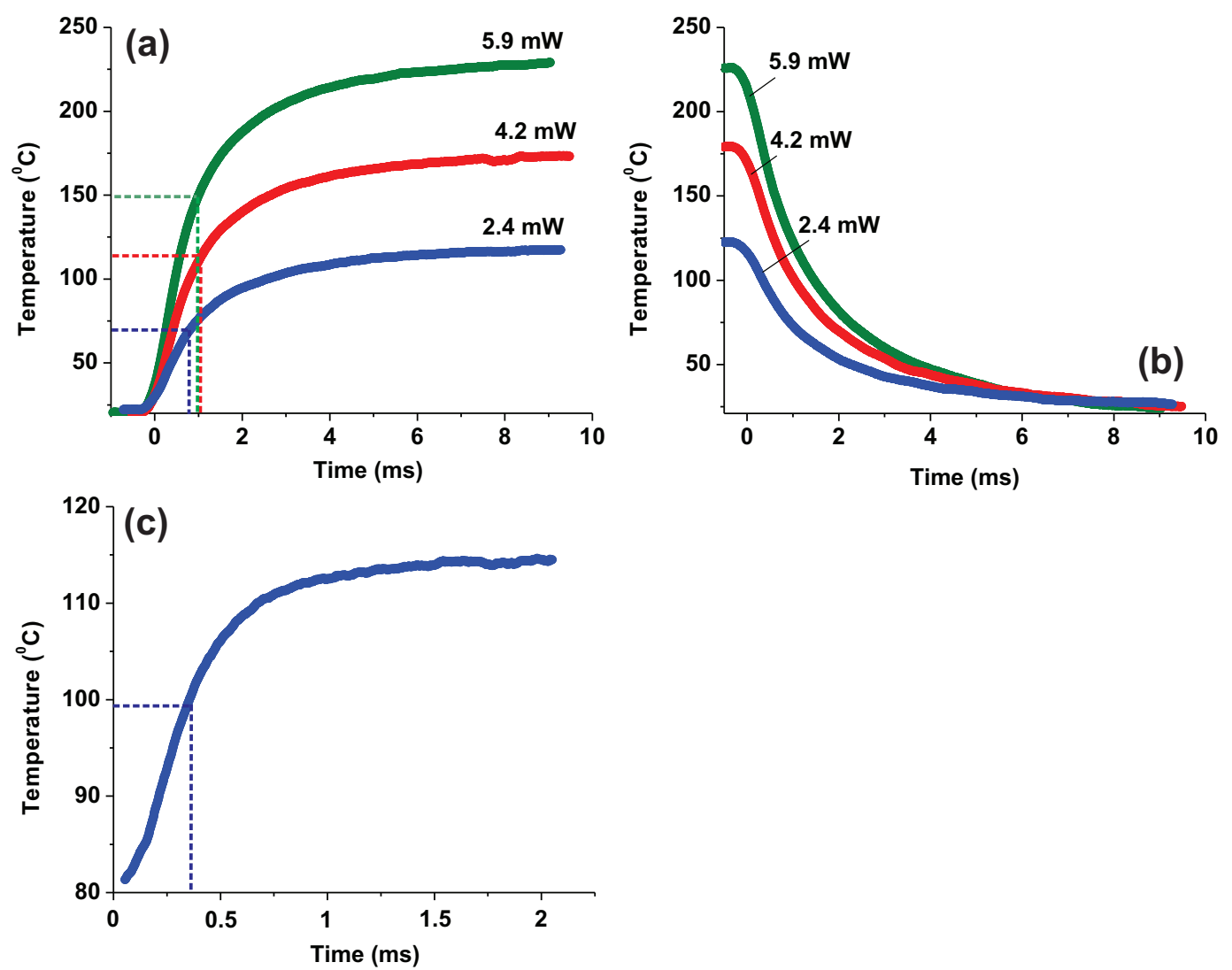

Figure 5.11: The heating (a), cooling (b) time constants measured for the TiSi 2 /Poly-Si temperature sensor when pulsing the poly-Si heater (circuit shown in Figure 5.2a) and the heating time constant(c) when pulsing the TiSi $2 /$ Poly-Si temperature sensor (circuit shown in Figure 5.2b). Rise time constants corresponding to 63.2 $\%$ are indicated with dash lines.

cation. Oxidation of these compounds proceeds at different initial temperatures and also results in the release of different amounts of heat. On the basis of these two parameters differentiation between those can be made. However, the low resistance of the temperature sensors (50-150 $\Omega$ ) in combination with the constant temperature measurement mode did not result in sufficient sensitivity to differentiate between these three compounds. In other words, the signal-to-noise ratio was too low (below 3) and reliable gas sensing measurements could not be performed. An example of the measurement performed with $0.5 \mathrm{vol} \%$ propane in constant flow mode is shown in Figure 5.12: resistance vs time (a) of the independent $\mathrm{TiSi}_{2} /$ poly-Si resistor and power output (b) of the PID loop. The resistance increase of $0.5 \Omega$ was measured, which is corresponded to temperature increase of $1.28{ }^{\circ} \mathrm{C}$. A power output is decreased on ca. $25 \mu \mathrm{W}$, however, estimation is difficult due to high noise in measurements. In stationary conditions, see Figure 5.13 measured with a Pt alumina supported catalyst (ca. $5 \mathrm{wt} \%$ ), the sensor is not cooled down by the flow, therefore a larger signal change was expected. Indeed, the difference between the reactive and non-reactive conditions is about $1 \mathrm{~mW}$ for the experiments performed above $300{ }^{\circ} \mathrm{C}$. However, this is still questionable whether the measured signal is attributed to the reaction only and not to temperature dependent thermo-physical properties of the gas mixture such as thermal conductivity and heat capacity. Also, different concetration of propane in air $(0.5 \mathrm{vol} \%$ and $0.25 \mathrm{vol} \%)$ did not result in power decrease. The reason could be that the actuating temperature was not sufficiently high. As an alternative, low temperature (starts at $100{ }^{\circ} \mathrm{C}$ ) reaction water formation in a non-explosive regime (shown in Figure 5.14) was selected. Detection of hydrogen was feasible already at $100{ }^{\circ} \mathrm{C}$, see Figure 5.15. 

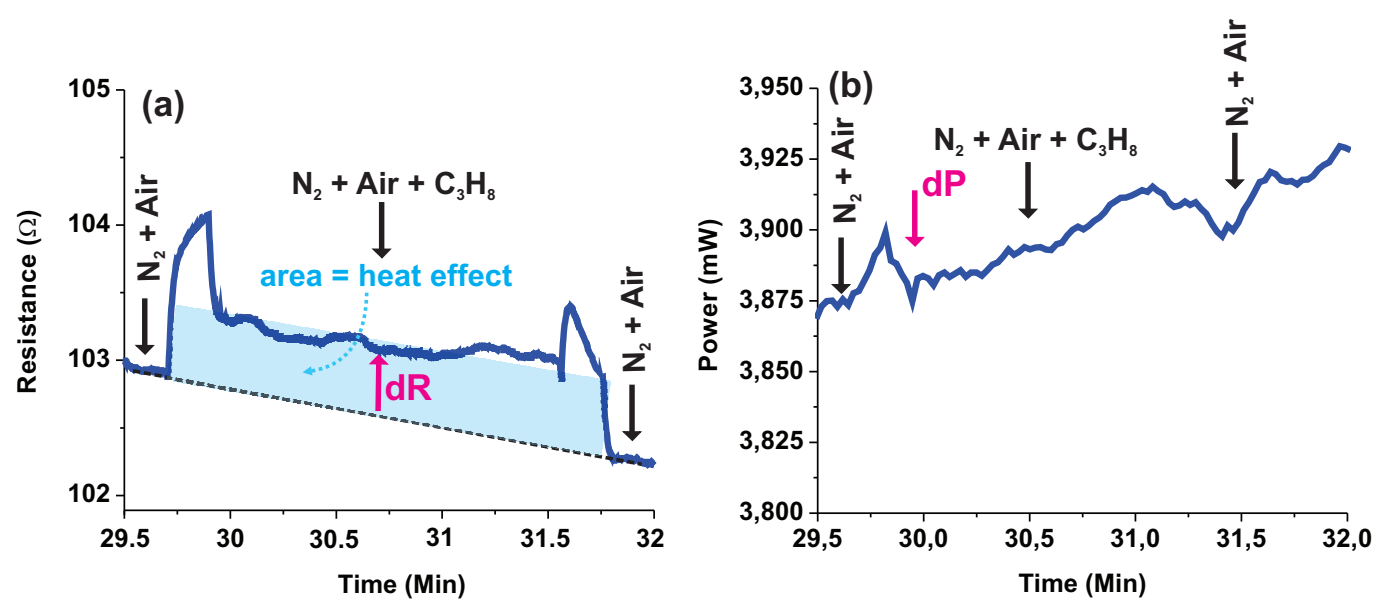

Figure 5.12: Sensor output measured in constant temperature mode at total flow of $3 \mathrm{sccm}(0.5 \mathrm{vol} \%$ propane, $10 \mathrm{vol} \%$ oxygen) and temperature kept $300{ }^{\circ} \mathrm{C}$ : resistance of the temperature sensor (a), power supplied to heater (b) collected in time. 5 wt \% Pt on alumina was used as a catalyst.

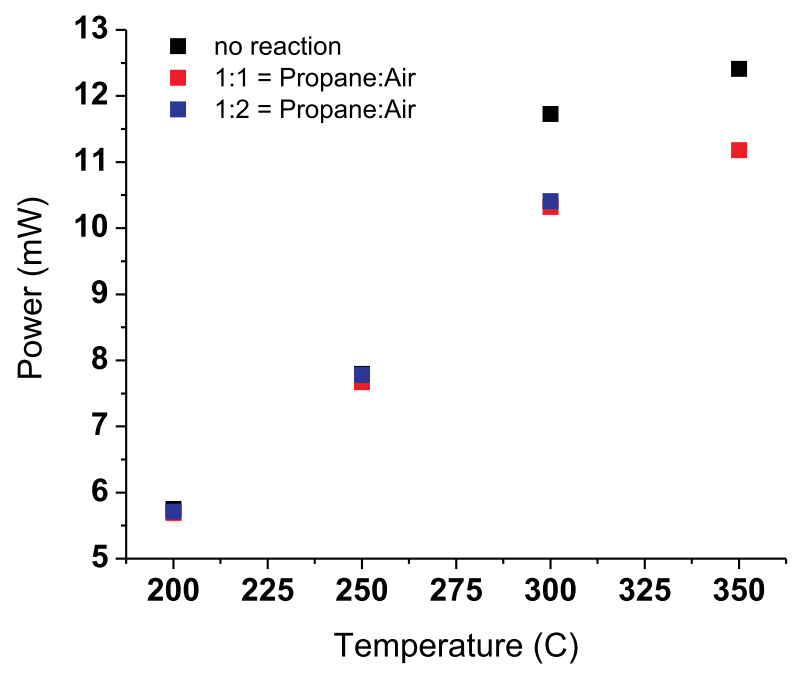

Figure 5.13: Sensor output measured in constant temperature mode and in non-flow conditions as a function of reaction temperature. Propane diluted in nitrogen and air were used. Three cases compared: no reaction conditions, 1:1 (0.5 vol \% propane, $10 \mathrm{vol} \%$ oxygen) and 1:2 (0.25 vol \% propane, $10 \mathrm{vol} \%$ oxygen) ratio of volume \% propane to air. The $5 \mathrm{wt} \%$ Pt on alumina was used as a catalyst.

Based on the experiments performed we observe:

- The reaction was not observed when using the sputtered catalyst (the conversion is low and the sensitivity of the system is not sufficient);

- Using a sol-gel based catalyst (Pt on alumina) both oxidation of propane and hydrogen could be observed.

- With the Pt on alumina catalyst, $3 \mathrm{sccm}$ total flow and $300{ }^{\circ} \mathrm{C}, 0.5$ vol $\%$ of propane in air was possible to detect in constant temperature mode, however, SNR was below 3, see Figire 5.11.

- In non-flow conditions, see Figure 5.13, 0.5 vol \% of propane in air was possible to detect, however no difference between $0.5 \mathrm{vol} \%$ and $0.25 \mathrm{vol} \%$ of propane in air was observed. 
Table 5.3: Oxidation reactions considered in this study.

\begin{tabular}{lccc}
\hline Parameter & Reaction & Enthalpy $(\mathrm{kJ} / \mathrm{mol})$ & Ignition temperature $\left({ }^{\circ} \mathrm{C}\right)$ \\
\hline \hline Propane, $\mathrm{C}_{3} \mathrm{H}_{8}$ & $\mathrm{C}_{3} \mathrm{H}_{8}+5 \mathrm{O}_{2}=3 \mathrm{CO}_{2}+4 \mathrm{H}_{2} \mathrm{O}+\Delta \mathrm{Q}$ & -2221 & 540 \\
Propene, $\mathrm{C}_{3} \mathrm{H}_{6}$ & $\mathrm{C}_{3} \mathrm{H}_{6}+41 / 2 \mathrm{O}_{2}=3 \mathrm{CO}_{2}+3 \mathrm{H}_{2} \mathrm{O}+\Delta \mathrm{Q}$ & -1911 & 458 \\
Propyne, $\mathrm{C}_{3} \mathrm{H}_{4}$ & $\mathrm{C}_{3} \mathrm{H}_{6}+4 \mathrm{O}_{2}=3 \mathrm{CO}_{2}+2 \mathrm{H}_{2} \mathrm{O}+\Delta \mathrm{Q}$ & -1958 & 454 \\
Hydrogen, $\mathrm{H}_{2}$ & $2 \mathrm{H}_{2}+\mathrm{O}_{2}=2 \mathrm{H}_{2} \mathrm{O}+\Delta \mathrm{Q}$ & -286 & $100-200$ \\
\hline
\end{tabular}

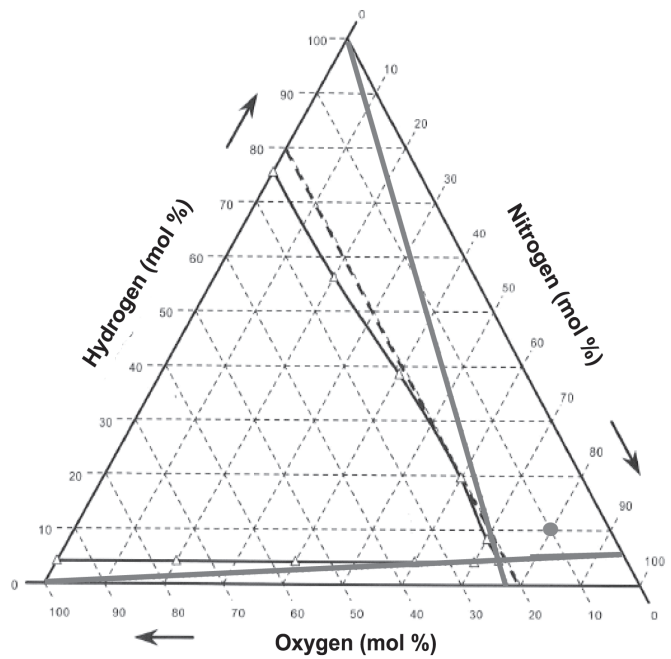

Figure 5.14: Diagram indicating safe (catalytically-driven) oxidation regime and the point selected in measurements.
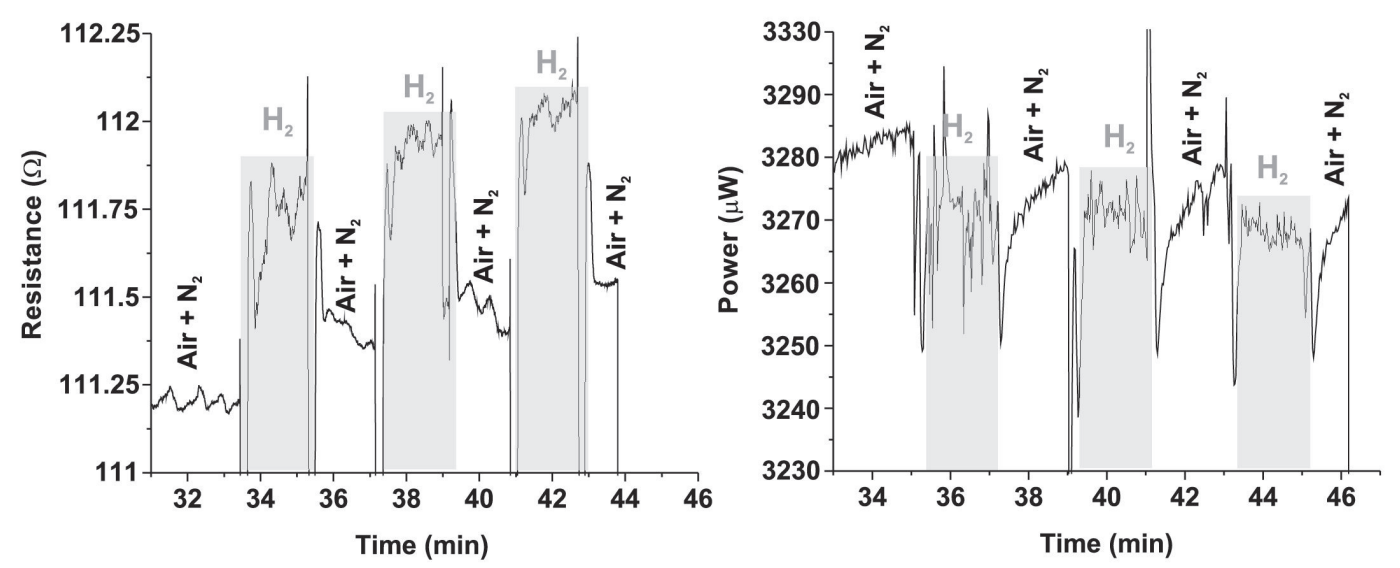

Figure 5.15: Response upon exposure to hydrogen of 5\% in constant temperature mode at $100{ }^{\circ} \mathrm{C}$. The total flow was kept constant and 3 sccm.

- It was possible to detect hydrogen 5 vol \% in air, see Figure 5.15 at total flow of $3 \mathrm{sccm}$ was possible to detect in the constant temperature mode at temperatures as low as $100{ }^{\circ} \mathrm{C}$ already. 


\subsubsection{Limitations of the current design and opportunities for future improvement}

The detection of reaction heats is a complex experiment requiring all individual constituents, such as micro hotplate, electrical read out, gas supply, catalyst to function reproducibly and without failure to minimize any system-induced errors. Here we list possible sources of failure in this experiment as well as limitations of the micro hotplates tested.

- Instability of the temperature in the lab affects the reference set point and increases uncertainty in measurements.

- The resolution of the temperature detector is controlled by the noise level and the capability of measurement equipment. Additionally, Johnson noise is always present in the temperature sensor.

- For the readout of electrical parameters we have used used instrumental multimeters. We did not use the Wheatstone bridge (WB), commonly applied: self heating of the WB disturbs the measurement of low-power signals. WB is a good solution for passive elements such as thermopiles, but not for our experiment, where the same resistive element is used for both heating and sensing purposes.

- Working with low flow rates (below $3 \mathrm{sccm}$ ) in microsystems is a challenge due to unstable reading of MFC-s. The error of MFC-s is equal to 1-2\% of total flow range and increases for lower flow rates. A possible solution is introduction of a restrictor after the MFC. A restrictor is a tubing with a known diameter and length; the diameter of the tubing shall increase the pressure after MFC-s and makes the flow more stable. Even with the restrictor MFC-s need a defined time to stabilize, which affects the experiment. It is better to work with higher flow rates, which can be achieved by introducing a bypass such as in flowmeters.

- Proper grounding is a problem in a complex setup. Each instrumental source-measure unit has a different ground level, when ground improperly causes ground loops.

A solution could be the use of a metal box for shielding. All the wires are coming and installed, i.e. grounded on the metal box, such that the tested chip inside is minimally affected by electro-magnetic interferences. Additionally, semiconductor-based hot-plates need to be tested in dark environment.

- Stabilization of the temperature of the active area of the sensor takes sometimes several hours. At temperatures above $400{ }^{\circ} \mathrm{C}$ the stabilization of the temperature is a challenge in our design.

- Microcalorimetric sensors have a tendency to fail, i.e. burn, when operated in temperature constant mode. This is due to overheating, which might be caused by the reaction, change in ambient conditions and thermal properties of the fluid.

- Membranes of $200 \mathrm{~nm}$ are good for heat localization, but not for catalyst deposition and performing catalytic reaction on top of them. Such membranes are too thin and mechanically instable and, thus, fail due to burning easily.

- The location of the heat source with respect to temperature sensors is important. The best would be to have temperature sensor on top or underneath the heater. In this case, all resistive structures are formed in one layer with the temperature sensor located in close proximity to the heater covered with the catalyst. There can be an optimized design to detect larger thermal changes, if the heater shape allows positioning of sensor in its center. The temperature distribution, if uneven, over the heater area influences the catalyst deposited which may enhance or retard operation of the sensor.

- The catalyst location with respect to temperature sensor and heater is also important. The catalyst should cover the temperature sensor completely. 


\subsection{Conclusions and Outlook}

The $\mathrm{TiSi}_{2} /$ poly-Si micro hot plate were characterized for application in microcalorimetric analysis of gaseous samples and the proof of concept operation in constant temperature mode was demonstrated on example of the propane oxidation $(0.5 \mathrm{vol} \%)$ and hydrogen oxidation $(5 \mathrm{vol} \%)$ in air.

The results of the thermo-electrical characterization showed that the devices are thermally efficient (can maintain $350{ }^{\circ} \mathrm{C}$ at $12 \mathrm{~mW}$ ), stable up to $800^{\circ} \mathrm{C}$ and response fast to the thermal changes on the active area (thermal heating times are within 1-2 ms), therefore suitable for detection of heats as a result of highly exothermic reactions. The materials implemented, i.e. poly-Si and $\mathrm{TiSi}_{2} /$ poly-Si meet all the necessary functional requirements. However, the design can be optimized further with respect to dimensions of the heater and catalyst, location of the temperature detectors relative to heater and catalyst and membrane thickness (see section 5.3.5).

The feasibility of the gas detection in the constant power mode, based on the feedback loop between the poly-Si heater and $\mathrm{TiSi}_{2} /$ poly-Si temperature sensor, was demonstrated on example of the sensor response to pulsing of propane $(0.5 \mathrm{vol} \%)$ and hydrogen $(5 \mathrm{vol} \%)$ in air. As expected upon exposure to oxidized gas the level of power supplied is decreased due to the release of heat.

As a part of the future study we propose to test current design in constant temperature mode while using the poly-Si heater only for the extraction of the sensor signal and the $\mathrm{TiSi}_{2} /$ poly-Si resistors specifically for temperature measurement and calibration. This might reduce the noise level (due to higher measurement resistor) and allow the comparison between the catalytic oxidation of propane, propene and propyne at temperatures in the range $200-600^{\circ} \mathrm{C}$, which was not possible to obtain up to now. However, the TCR of poly-Si is substantially lower compared to $\mathrm{TiSi}_{2} /$ poly-Si, which also affects the measurements.

The detection of hydrogen was possible already at $100{ }^{\circ} \mathrm{C}$ for $5 \mathrm{vol} \%$. In the future we plan to test it at various concentrations (below $5 \mathrm{vol} \%$ ) and temperature range $\left(50-200{ }^{\circ} \mathrm{C}\right)$. The challenge for this reaction is that thin membrane sensors are easily burned due to the overheating. 


\section{6 Ultra-low power titanium nitride hot surface devices.}

An alternative concept for micro- and nano-scale hot surface devices with ultra-low power consumption is introduced.

The hot surface devices consist of a thin film - titanium nitride (TiN) - resistive element in a 4-point configuration: meander-, spiral- or link-shaped heater. They can facilitate local heating and temperature sensing of an area $0.001-0.1 \mathrm{~mm}^{2}$ up to $300^{\circ} \mathrm{C}$ at a power consumption of less than $3 \mathrm{~mW}$, and for particular designs even limited to a few $\mu \mathrm{W}$.

This enables potential applications in various portable sensing systems, where rapid temperature sensing and/or thermal actuation with high spacial resolution and minimized power consumption are required.

Ultra low power, CMOS compatible, suspended membrane, hot surface devices were proposed and preliminary tested as transducers for calorimetric sensing applications as flow sensor, as thermal conductivity detector and as catalytic gas detector (pellistor). The results of these experiments are discussed. Operation as a flow sensor and as a thermal conductivity detector was demonstrated. It was, however, not possible to obtain reliable measurements while operating the link-based hot surface devices as a pellistor. Based on conducted measurements and electrical characterization, conclusions are drawn on feasible applications as well as inherent limitations in the operation of these devices.

${ }^{*}$ This work has been conducted in collaboration with Chair of Semiconductor Components, University of Twente. 


\subsection{Introduction}

Microscale hot surface devices are important transducer elements for a variety of sensing and analytical applications.

However, very often the power consumption of such systems is too high $(50-100 \mathrm{~mW})$, and their dimensions do not enable integration on-a-chip using CMOS-compatible technologies. Additionally, the spacial resolution of commonly used micro-hotplates is usually limited to tens of microns. This retards their implementation to a range of applications where rapid extraction of the temperature signal from objects of a few nanometers is desired [18].

Downscaling of the hot surface devices, is, therefore, an important step towards accurate measurement of temperature at the low micro- and nanoscale range. When miniaturized devices are realized with CMOS standards, this allows a further upgrade to complex Si-based sensing systems and/or the addition of electronic control on a single chip.

Nowadays, thermal sensors allow the measurement of temperature changes with a resolution down to $10^{-4} \mathrm{~K}$. Electrical amplification can increase the sensitivity, but makes the setup complicated, bulky and cost inefficient. Another way to achieve a higher resolution and a rapid response is by using physical amplification, i.e. downscaling of the dimensions of the heater and sensor.

For these reasons, there is a great demand for hot surface sensors and actuators with the following specifications:

- Minimized energy consumption;

- Local measurement and thermal actuation;

- Ultra-fast operation;

- Simple and cost efficient realization using Si-based processes;

This chapters concerns the utilization of a novel type of hot surface devices as transducers for various calorimetric applications such as flow sensing, thermal conductivity measurement, pellistor. In the past, the operation of similar structures (the link was formed by the breakdown of underlying dielectric) as chemical sensors was successfully demonstrated by Kovalgin et al. [147].

\subsection{Flat vs. link-based heaters}

The devices are based on thin film resistive elements of TiN that are designed in different shapes:

- Flat meander-shaped;

- Flat spiral-shaped;

- Micro- and nano- scopic conductive link.

Flat meander and spiral structures belong to the group of conventional heaters, where dissipation of energy occurs in the highly resistive part (see Figure 6.1a). The shape and design of connecting leads allows to achieve a local heating, but at the same time such large leads also enable losses to the environment. The motivation for the design of meander and spiral heaters was to test the feasibility of ultra-thin TiN layers as a novel resistive material, and to implement these devices for calibration of micron link-based heaters. The ultra-thin TiN is characterized by high thermodynamic stability and high corrosion resistance, therefore an interesting material to implement in hot surface devices for monitoring of high temperature oxidation reactions. The properties of thin TiN are strongly dependent on deposition method, in this case Atomic Layer Deposition (ALD) from $\mathrm{TiCl}_{4}$ and $\mathrm{NH}_{3}$ at $425{ }^{\circ} \mathrm{C}$ in the cluster system developed in the Semiconductor Components Group (University of Twente).

Meander- and spiral-shaped thin TiN resistors were used as a calibration standard for micron- and nano- link 
devices. The reason for this is that infrared imaging techniques, for instance, are limited to a few micron large emitting objects, so that larger devices are needed as a reference. Extraction of these data enables the possibility of comparing the performance of conventional and link-based resistors. A link-based heating and
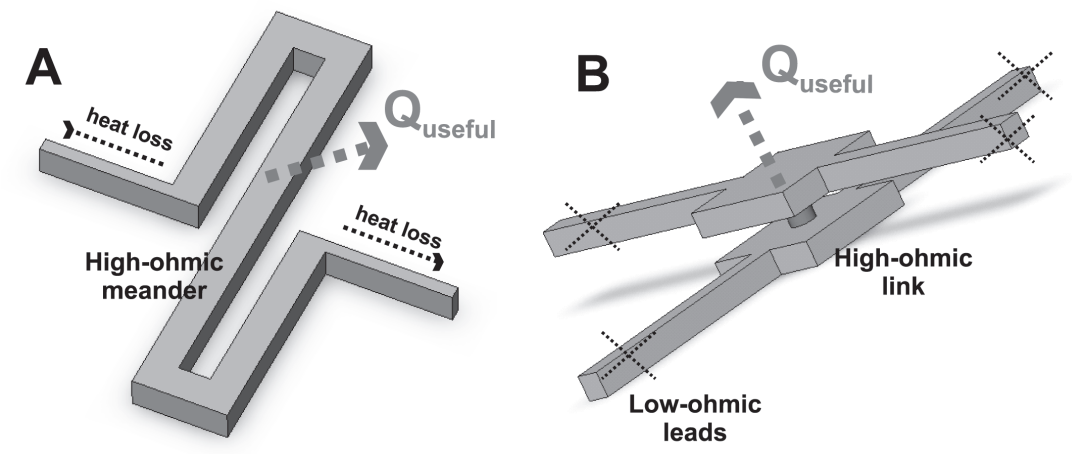

Figure 6.1: Schematic representation of conventional meander heater (a) and novel link-based heater (b) with heat loss path.

sensing device (see Figure 6.1b) is based on a small and highly conductive link which can act both as heater and temperature sensor. This is a novel type of heater where the dissipation of heat takes place inside the link acting as a resistor. In such a structure the electrical and thermal resistances are controlled independently. This reduces conduction losses through the resistive leads, which is a problem for all flat heaters, as the leads remain "cold" during the device operation. Reduced losses increases the overall efficiency of the heating device. The motivation for the design of link-based devices is the realization of a concept that allows the achievement of higher heating and temperature sensing efficiencies at a few $\mathrm{mW}$ power. An important property of link-based heaters and temperature sensors is that they provide information on the local heat effect. Moreover, link-based devices with a power consumption of a factor 1000 lower than state-of-the-art low power micro-hotplates can become a valuable element of various sensing and actuating systems.

\subsection{Overview of designs and fabrication}

In this section a brief overview of the developed designs and fabrication flow are discussed. For more details refer to work of A. Groenland [148], [149]. Three types of the hot surface devices were developed with typical dimensions and corresponding range of resistances listed in Table 6.1:

- Flat meander and spiral heaters (see Figure 6.2);

- Micron and nano- link-based devices (see Figure 6.3).

The meander and spiral hot surface devices consisted of a highly resistive TiN structure deposited by atomic layer deposition (ALD) and thick sputtered TiN electrodes and contacted through an isolating layer $\left(\mathrm{SiO}_{2}\right)$ with layer of Aluminum (Al). The layer-by-layer design and cross section through the active sensing area are schematically demonstrated in Figure 6.2(a-d). For the pellistor application, the hot surface area was covered with $100 \mathrm{~nm}$ Pt catalyst (with $15 \mathrm{~nm}$ Ta used as an adhesion layer) as shown for a spiral shaped heater in Figure 6.2e. Both resistors are contacted with pairs of current sourcing and voltage measuring leads for precise extraction of electrical parameters.

The design outlook for link-based devices is shown in Figure 6.3. The two crossing electrodes are separated by an isolator $\left(\mathrm{SiO}_{2}\right)$. The top and bottom electrodes are $100 \mu \mathrm{m}$ sputtered TiN forming force and sense leads to an active, heat transmitting surface of the link. The link itself was filled with 7-10 nm ALD TiN. Diameter of the link was varied in the range $50 \mathrm{~nm}$ to $23.5 \mu \mathrm{m}$. Nanoscale links were defined using e-beam lithography. Links in the range 2 to $23.5 \mu \mathrm{m}$ were defined by UV lithography. For the pellistor application a catalyst thin film was immobilized on the high ohmic part, i.e. link. The size of catalyst varied from 10 to $100 \mu \mathrm{m}$ in diameter. 

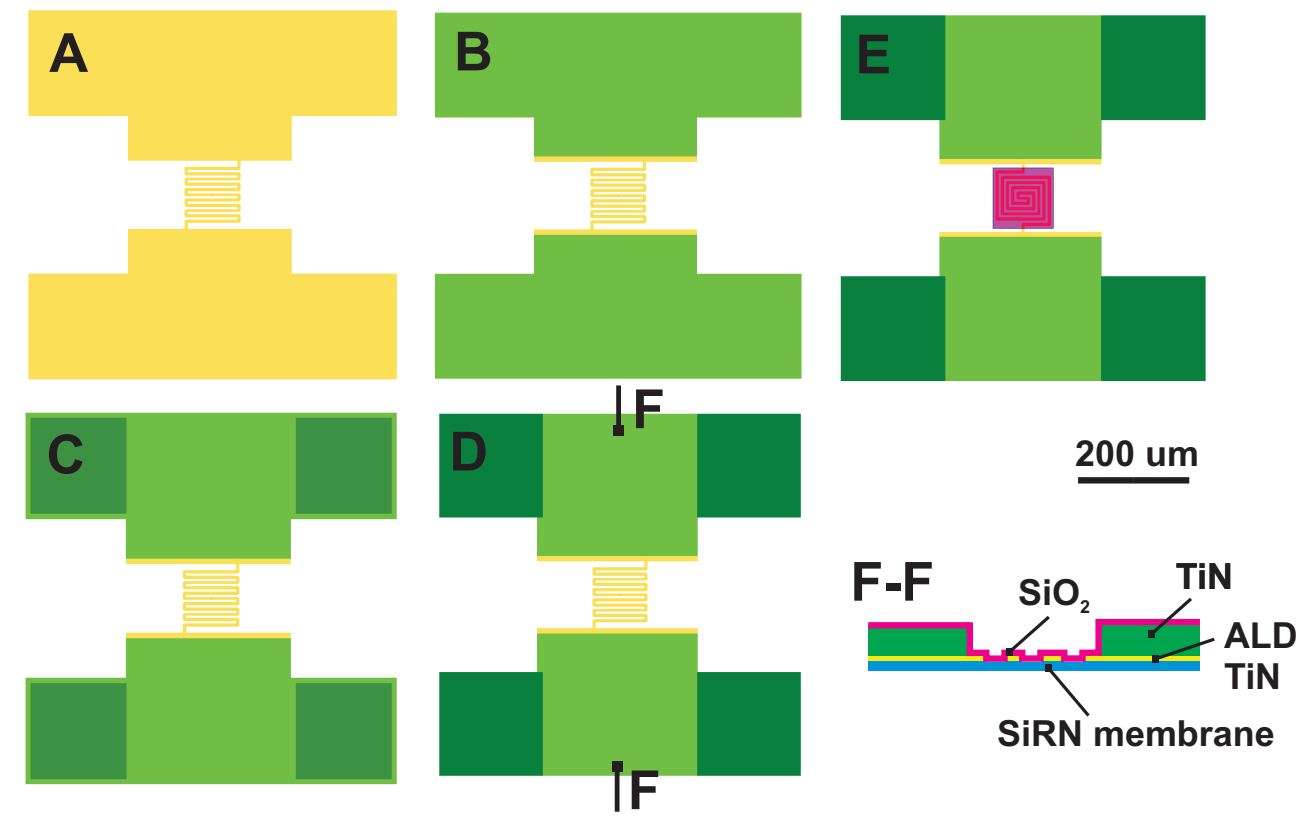

Figure 6.2: Design schematic for meander-shaped TiN ALD heater: (a) 7-10 nm ALD TiN, (b) $100 \mathrm{~nm}$ sputtered TiN, (c) openings through $\mathrm{SiO}_{2}$ for metalization, (d) Al metalization layer, (e) spiral-shaped heater with deposited thin film catalyst, $(f)$ cross sectional view.
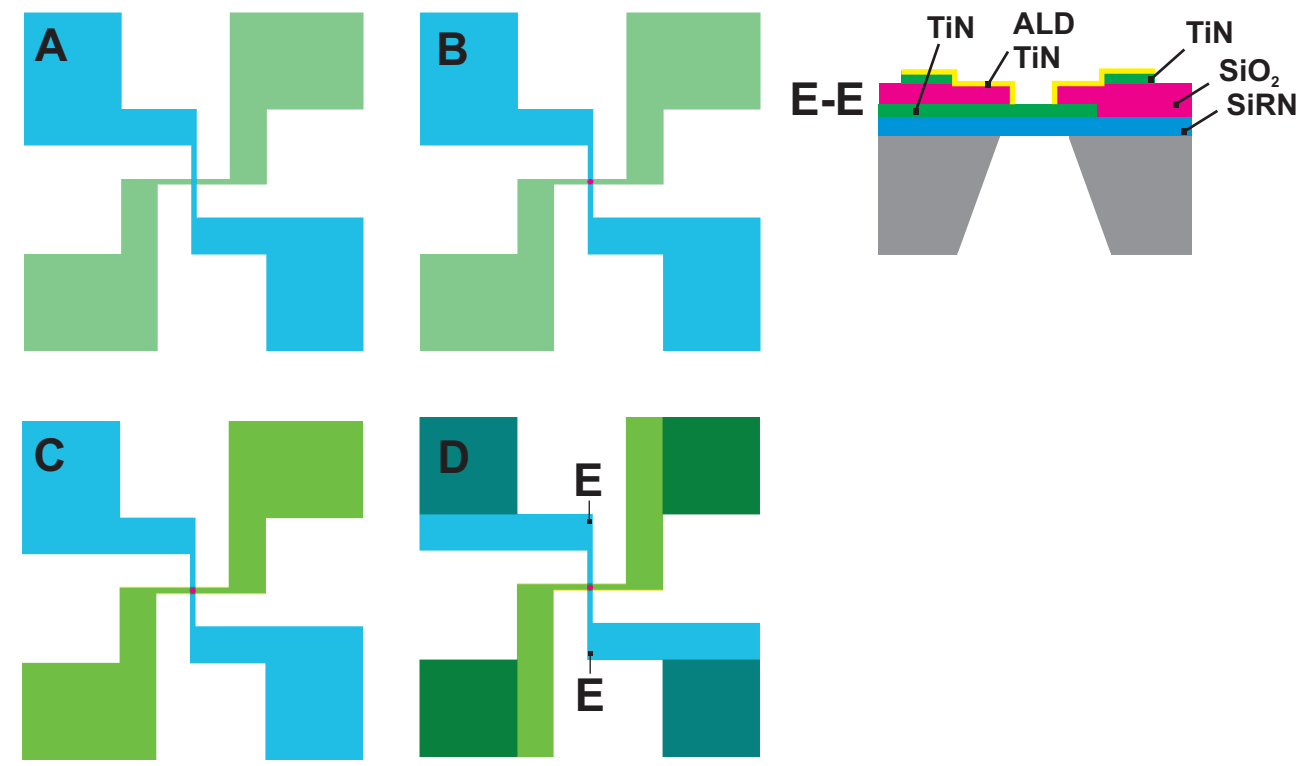

Figure 6.3: Design outlook for link-based heater: (a) bottom and top TiN sputtered electrodes separated by $\mathrm{SiO}_{2}$ insulator, (b) defined area of high-ohmic link, (c) 7-10 nm ALD TiN filling the link, (d) Al metallization layer (e) cross-sectional view.

All types of devices were made on a dielectric membrane with a suspended area of $110 \times 110 \mu \mathrm{m}^{2}$. This was done to achieve local heat confinement at reduced power consumption. The difference between the operating principles of conventional and link-based heaters is schematically demonstrated in Figure 6.1. In order to maximize the local heating effect, usually the dimensions of the leads are much larger compared to the high ohmic part. This, however, results in an increased conductive heat flux from the leads to the environment. A balance has to be found between electrical and thermal conductive properties of the leads. This is a challenging task in case of flat heaters (meander, spiral): resistances of high-ohmic and low-ohmic parts are comparable 
Table 6.1: Dimensions and corresponded measured range of resistance of the hot surface devices.

\begin{tabular}{lcc}
\hline Device & Heated area $\left(\mu \mathrm{m}^{2}\right)$ & Resistance $(\Omega)$ \\
\hline \hline$\mu$ m-SMA & $3.14-28.26$ & up to $10^{4}$ \\
nm-SMA & $1.9 \cdot 10^{-3}$ & up to $10^{6}$ \\
Meander & $110 \times 110$ & $14000-40000$ \\
Spiral & $110 \times 110$ & $14000-40000$ \\
\hline
\end{tabular}

and leads do not remain "cold" during the device operation. In contrast, in a link-based device the ratio of link resistance to resistance of leads can be matched (by means of various link designs, selection of highly resistive materials, modification of isolating layer, etc.) in such a way that the leads will remain unheated and losses by conduction through the leads can be reduced, resulting in a higher thermal efficiency. Losses by conduction through leads is one of the main heat loss mechanisms in such systems, besides conduction to the silicon substrate and the environment.

\subsubsection{Thermo-electrical characterization}

When a heating element is used to monitor the temperature change in an ambient or on a hot surface (as a result of physical and/or chemical interaction), its sensitivity is determined by the dependence of the resistance on temperature. The measured sensor signal from a heater also depends on the power dissipated during the measurement and on the thermal dynamics.

In this section the main thermo-electrical properties, i.e. the temperature coefficient of resistance (TCR), the dependence of the surface temperature on power consumption (PT curve) and the thermal time constant are discussed.

\section{Temperature dependence of resistance}

The resistance vs temperature characteristics of TiN ALD thin film-based hot-surface devices can be roughly described by a linear relation with a characteristic slope $\beta$ which when divided by the resistance value extrapolated to $0{ }^{\circ} \mathrm{C}$ gives the TCR $(\alpha)$.

$$
R=R_{0}\left(1+\frac{\beta}{R_{0}} T\right)=R_{0}(1+\alpha T),
$$

where $\alpha$ is the TCR and $R_{0}$ is the resistance at $0{ }^{\circ} \mathrm{C}$. In Figure 6.4 the resistance as a function of the temperature is presented for meander, spiral and link-based heaters collected in the range from room temperature to $300{ }^{\circ} \mathrm{C}$. Both a positive and a negative TCR were found. The variation in TCR between flat and link heaters can be attributed to the different content of oxygen in grown TiN thin films or the influence of contact resistance. In addition to that, variations in the processing of ALD TiN thin films between the first and the second run resulted in a change in the TCR sign. Over the computed range of devices indicated a variation in TCR values from ca. $-2 \times 10^{-3}$ to $+2.5 \times 10^{-4} /{ }^{\circ} \mathrm{C}$ was found.

\section{PT curve}

Presented in Figure 6.5 is the dependence of temperature of different hot surface devices on supplied power with a maximum temperature being ca. $250-300{ }^{\circ} \mathrm{C}$. As can be seen, nanolink-based heaters require only 5 $\mu \mathrm{W}$ to obtain a temperature above $250{ }^{\circ} \mathrm{C}$. For such a temperature, micron-sized link heaters consume up to $0.5 \mathrm{~mW}$, and a flat meander up to $8-10 \mathrm{~mW}$. This gives a thermal efficiency $5 \times 10^{4}{ }^{\circ} \mathrm{C} / \mathrm{mW}$ for the nanolink, $500{ }^{\circ} \mathrm{C} / \mathrm{mW}$ for the micron-link and $25^{\circ} \mathrm{C} / \mathrm{mW}$ for flat heaters. The efficiency of the latter is comparable to values mentioned in literature for thin film suspended heaters [150], which is within the range of tens ${ }^{\circ} \mathrm{C} / \mathrm{mW}$. The heating efficiency of nano- and micro- link devices is much higher compared to state-of-the-art hot surface devices. 

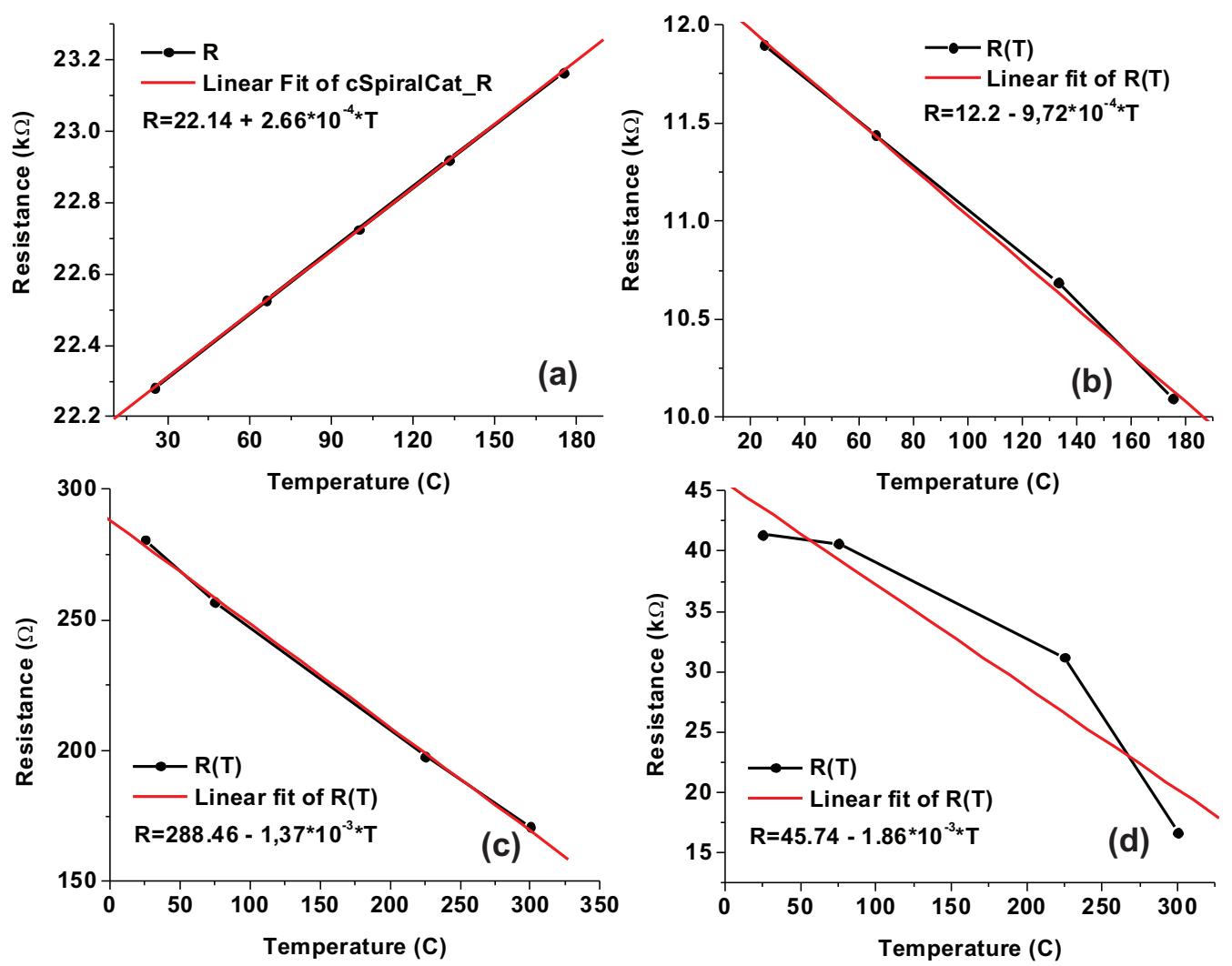

Figure 6.4: Temperature dependence of resistance for spiral ALD TiN heater (a) and $\mu m$-SMA (b) from run 2 (w175), for nm-SMA (c) and meander heater (d) from run 2 (w181).

\section{Thermal time constant}

It takes a defined amount of time for a heating element to adjust its temperature when conditions are changed suddenly. To estimate this effect the thermal time concept is implemented, i.e. the time needed for a heater to change its resistance to a value of $63.2 \%$ according to a baseline value. This time is required to be within a ms (or a few ms) for rapid sensing and heating applications. To quantify the dynamic response of a heating element to power pulses, the thermal time constant was measured in open air. In Figure 6.6 shows the dynamic response of a $\mu \mathrm{m}$-link-based and flat meander heaters obtained as a result of a square voltage pulse of $1.2 \mathrm{~V}$ and $6.6 \mathrm{~V}$, respectively. As can be seen, the rise time is less than $0.5 \mathrm{~ms}$. We would expect the link-based devices to exhibit smaller thermal time constants, but surprisingly, flat ALD TiN heaters were found to respond within $\mu$ s range. The measured link-based devices are low ohmic and, therefore, the resistance is governed not only by the link but also by the resistive leads, which increases the overall thermal mass. To sum up, both link-SMA and flat meander heaters exhibit small thermal time constants (within $0.5 \mathrm{~ms}$ ) which makes rapid sensing application feasible.

\subsection{Selected applications}

In this section an overview of applications for the studied devices will be given. For each type of sensing technology a correlation between measured output parameters and physical or chemical process will be discussed. In the simplified form a relation describing the thermal output of a heating element used as flow, thermal conductivity, or catalytic sensor can be expressed as:

$$
Q_{\text {in }}+Q_{\text {cat }}-Q_{\text {loss }}=Q_{\text {out }}(R, U, P)
$$



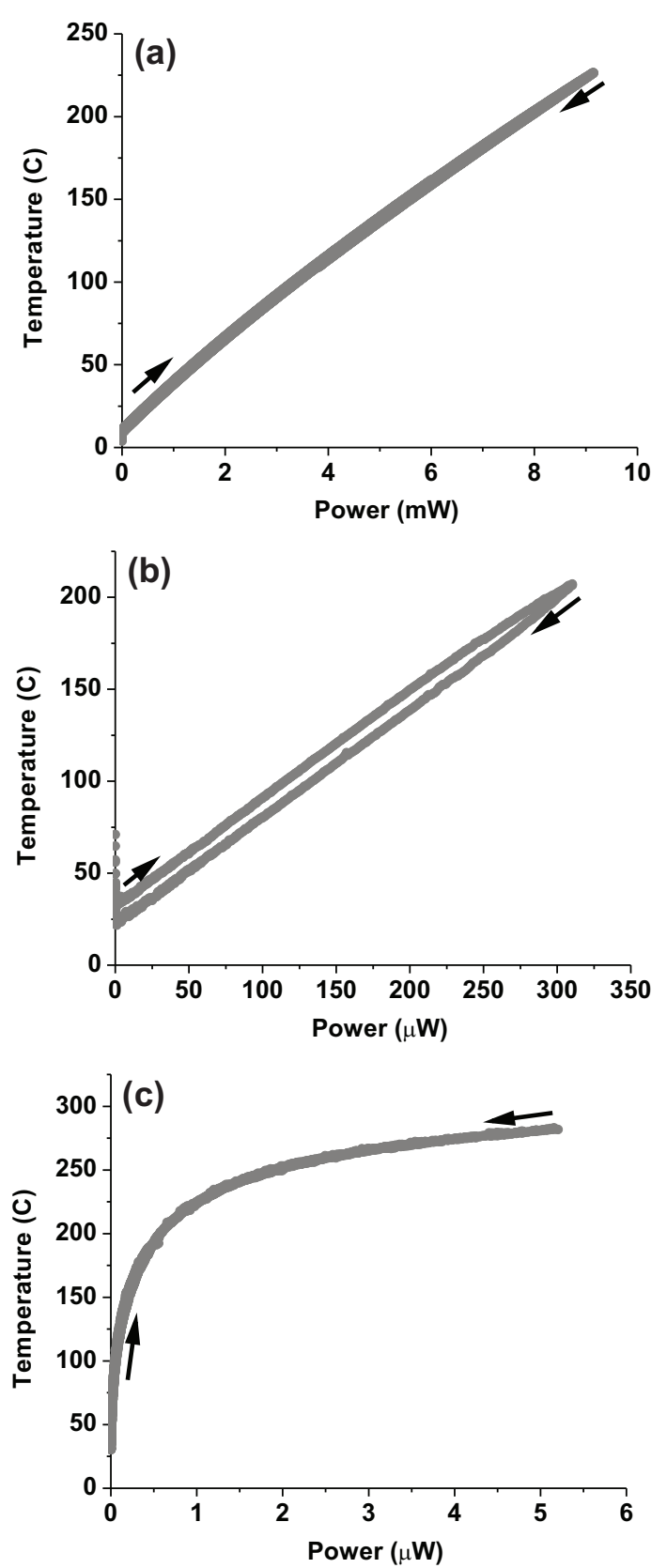

Figure 6.5: Dependence of temperature maintained at the hot surface on supplied power for $(a)$ meander, $(b)$ micro link and (c) nano-link heaters.

Where $Q_{i n}$ is the supplied power expressed as measured voltage across the heating element multiplied by the current passing through it:

$$
Q_{\text {in }}=U \cdot I
$$

$Q_{c a t}$ is the heat release/uptake due to the catalytic reaction (adopted from [151]):

$$
Q_{c a t} \sim B \cdot D \cdot \Delta_{r} H^{0} \cdot[L E L]
$$

In Equation 6.4, the constant $B$ is a function of the catalytic sensor design and assembly (related to the thermal resistance of the construction to the environment, e.g. $m \times C_{p}$ ) and catalyst properties (volume, concentration 

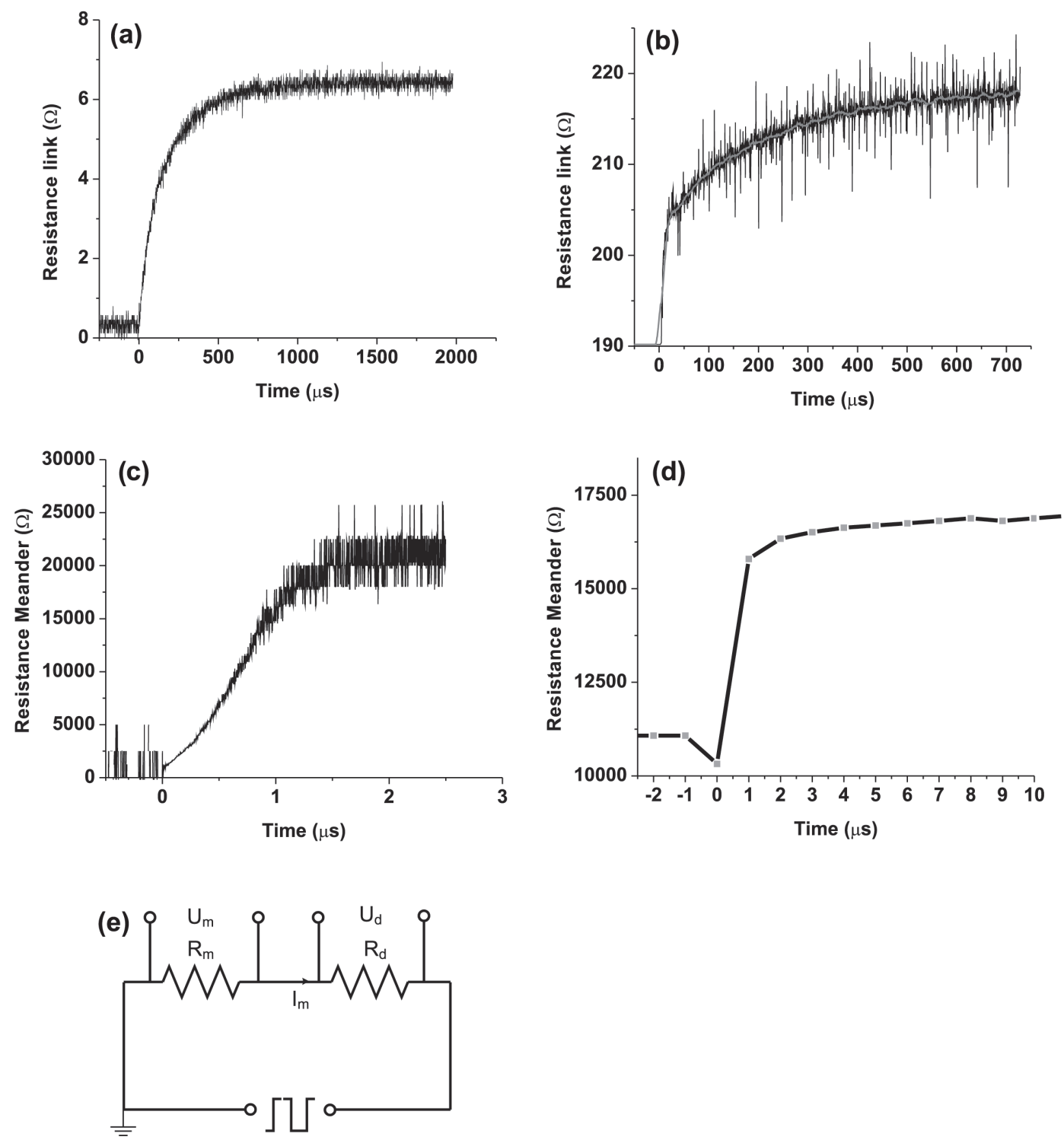

Um, Rm - measurement resistor

Ud, Rd - tested device (link, meander)

Figure 6.6: Thermal time constants measured $\mu$ m-link-based devices (a-b) (w175) and meander flat heaters $(c-d)(w 175)$, measurement circuit used (e).

of active sites); $D$ is the diffusion coefficient to active sites, $\Delta_{r} H^{0}$ is the standard enthalpy of the reaction, [LEL] is the lower explosive limit of the detected gas in air. An estimation of $Q_{c a t}$ is done relative to methane, which is usually considered as a standard gas (if data are available), in order that $k$ does not need to be considered.

$Q_{\text {loss }}$ represents thermal heat losses, e.g. radiation, convection (natural, forced), conduction (in both solids and fluids), and can be expressed in a simplified approximation as a function of the temperature difference relative to ambient $\left(\mathrm{T}_{a m b}\right)$ and thermal conductivities $(\lambda)$ of all constituents of the system:

$$
Q_{\text {loss }} \sim f\left(T-T_{a m b}\right) \cdot f(\lambda)
$$

$Q_{\text {out }}$ represents the measurable thermal response of the heating element and depending on the selected measurement circuit, can be either resistance, voltage across or power level of a heating element. 


\subsubsection{Calorimetric flow sensor}

Calorimetric flow sensors are widely applied for flow metering of both gaseous and liquid media in a broad flow range, as well as for the detection of movement, e.g. via flow-/no-flow applications. When flow passes the hot surface, it causes distortion of the temperature profile because of the enhanced conductive and convective heat fluxes (losses) as described in Equation 6.5. The sensing mechanism is, thus, based on measurement of heat removal from the heated element due to the flow or flow variations. Preferably, the sensing curve is a linearized dependence of the sensor output signal (relative to a stationary situation or reference flow) on flow.

\subsubsection{Thermal conductivity detector}

Thermal conductivity detectors are commonly used in gas/liquid chromatography. Various media have different thermal conductive properties. Usually there is a stable heat flux present from the heating element when in operation. When an analyte of interest with different thermal conductive properties is brought in contact with the hot surface, the heating element is heated up or cooled down because of enhanced or decreased thermal losses from the hot surface (Equation 6.5). The rate of the cooling/heating is related to a measurable electric output of the sensor and can be calibrated. Additionally, the output of the sensor is dependent on the diffusive properties of the analyzed gas, which influences how fast heat removal processes can take place. The signal is usually measured in constant temperature mode as a difference of output to a reference flow and an analyte flow. The concentration of an analyte can be determined by integrating the area of the peak.

\subsubsection{Catalytic detector of flammable gases}

Catalytic thermal sensors are used to detect the presence of combustible gases in air within the low explosive range (\% LEL). A thin film of catalyst integrated on the surface of a heating element causes the gas to react, and this provides a way for selectivity and lowers the operational temperature of the device. The release or uptake of thermal energy due to the combustion reaction causes an increase or decrease of the temperature of the heating element. The measurable output of the sensor is a decrease or increase in resistance (depending on the sign of the TCR) of the heating element for a constant power measurement circuit, or a difference in the level of supplied power in case of constant temperature mode operation.

\subsection{Experimental}

\subsubsection{Device assembly}

The typical die containing two devices in the middle for gas sensitivity tests (with and without the catalyst film) and test structures for process control is shown in Figure 6.7. The implemented device assembly, holder with installed spring connectors for electrical readout and glued capillaries, is demonstrated in Figure 6.8.

The design of holder ensures that the sensors located in the central area along the channel (two sensors with and without the catalyst) are exposed to the gas flow. The dimensions of the channel are chosen to provide a sufficient amount of reactants to the sensor and ensure controlled gas feed (laminar flow) and, thus, a better control over sensing conditions. Peek flexible tubings were used for the gas feed. The inner diameter of the tubings is $800 \mu \mathrm{m}$ to reduce the pressure drop over the inlet of the channel, and the outer diameter of the tubings was 1/16 inch (1587.5). Gold spring connectors were installed directly into the holder as it is an isolating material. Electrical connections were soldered on top and extended further to the measurement equipment. Alternatively, glass chip with etched channel profile glued to silicon chip using UV curable epoxy can be used. In this way high-temperature anodic bonding, that might cause a device failure (due to for instance oxidation of thin ALD films) is avoided. 


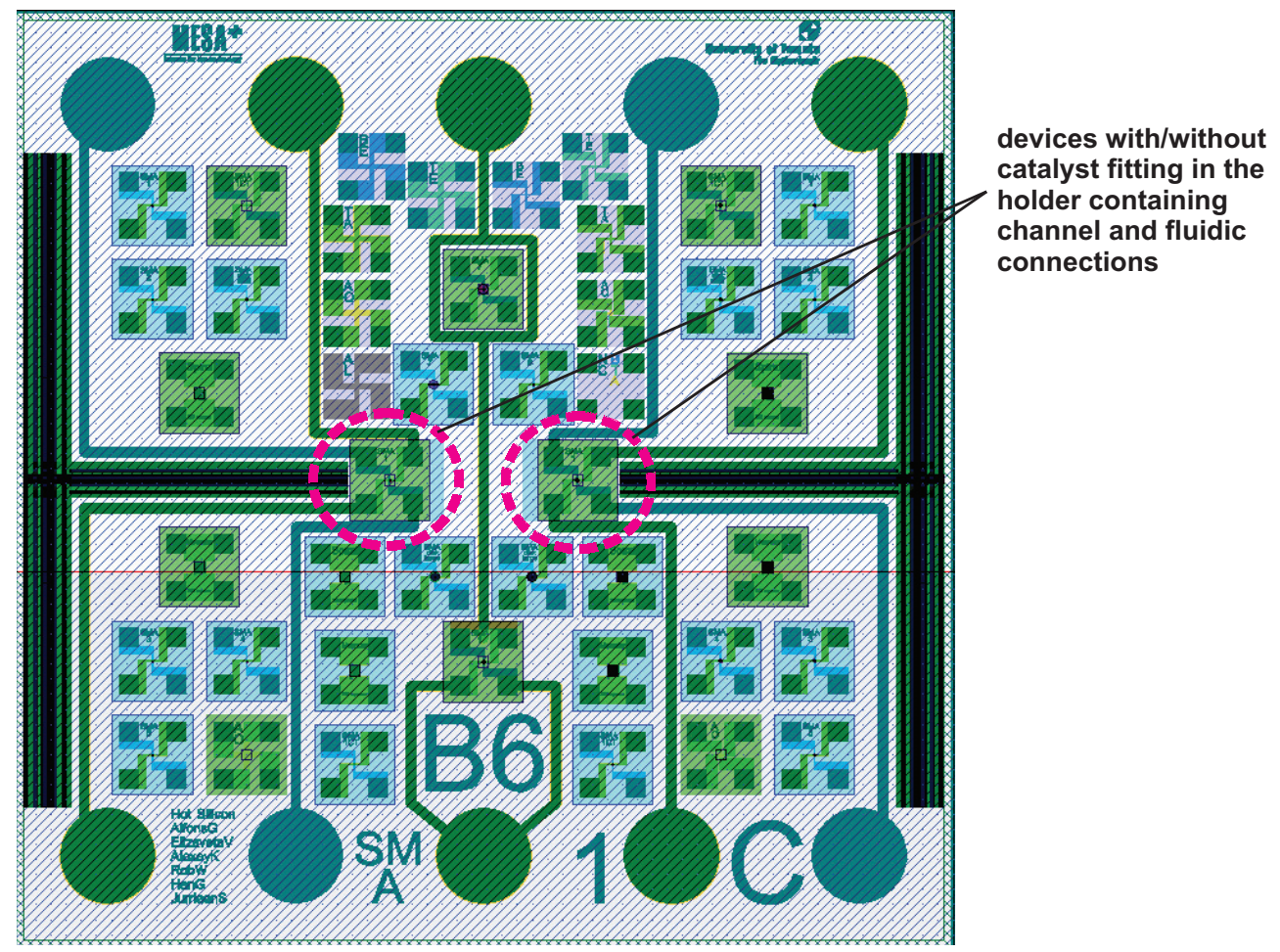

Figure 6.7: Outlook of the typical die containing two devices fitting in the holder channel for gas sensitivity tests and other test structures for process control.
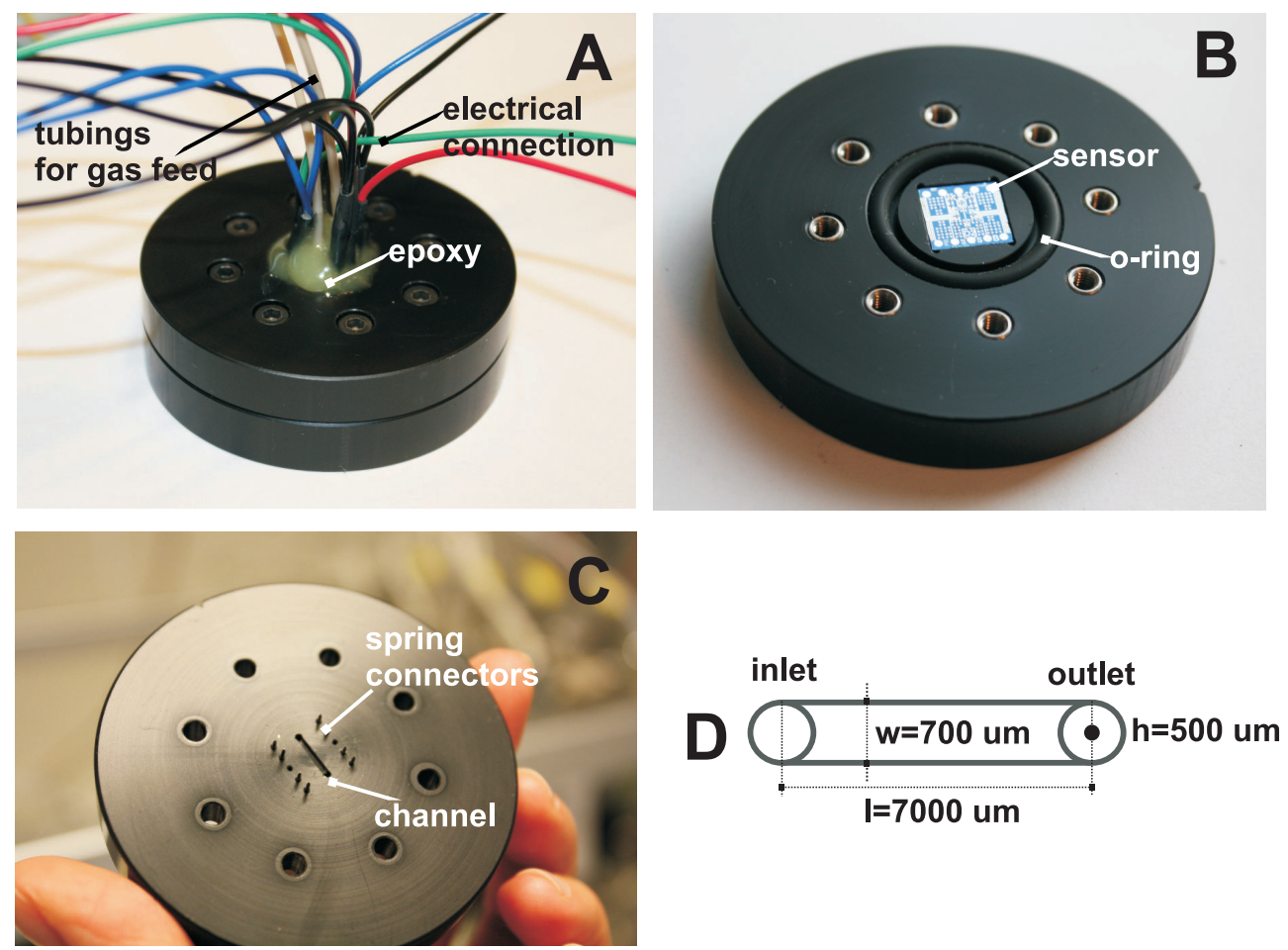

Figure 6.8: Assembly of the non-bonded chips in a holder (a), bottom part with the place for chip and o-ring ensuring leakage free connection (b), upper part containing the channel, inlet/outlet for gas feed and electrical connectors $(c)$, dimensions of the channel $(d)$. 


\subsubsection{Electrical readout}

Two types of signal readout modes were implemented:

- Constant current mode;

- Constant temperature mode.

The measurement circuit used is shown in Figure 6.9. In the constant current mode, the current set point was given to the heating element and the voltage across it was constantly measured. The changes in the measured voltage output is a measure of a distortion of the heat profile of the device. In constant temperature mode, a

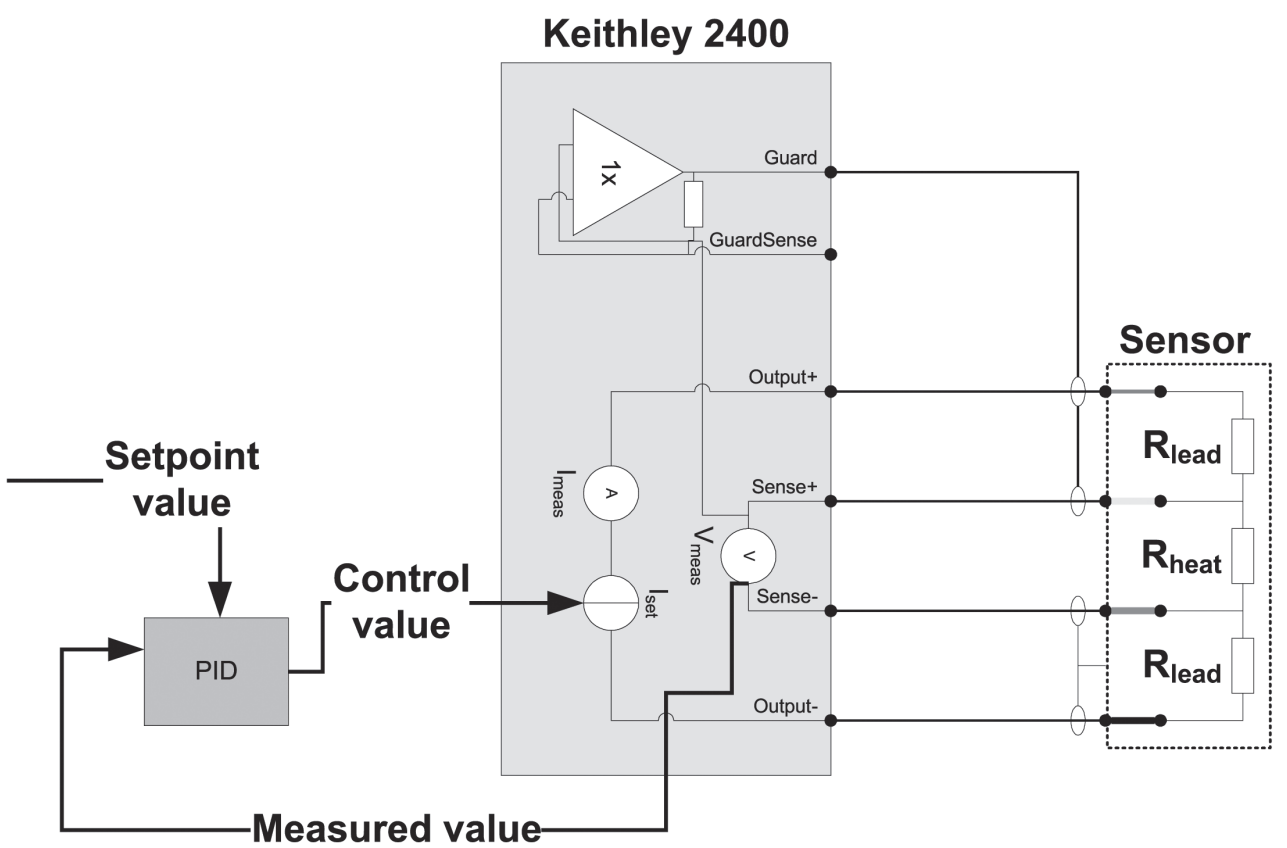

Figure 6.9: Measurement circuit used for readout of sensor signal.

temperature set point was given and a Labview-based feedback control was used to keep the heating element at a desired temperature. The control constantly adjusted the source current according to the temperature set point. Thus, an instantaneous change in the power level is a measure of the distortion due to heat profile change of the device. Here we assume that the resistance of the heating element, and thus the temperature, is changed according to Equation 6.1 with a constant TCR. If the TCR of the heating element is not stable along the measurement range, the feedback loop can not control correctly for the temperature. In this case, application of correction values for each temperature range is required. The arrangement of the gas supply was discussed in the previous chapters (see Chapter 3 and Chapter 5).

\subsection{Results and discussion}

\subsubsection{Flow sensor}

In Figure 6.10a a normalized output of the flat meander type of heating element upon exposure to nitrogen flow in the range 0 to $5 \mathrm{sccm}$ is shown. The sensor output - flow curve can be linearized with a good approximation, i.e. $\mathrm{R}^{2}=0.998$. A similar trend was obtained for a flat spiral heater (The measurements were performed at about $20 \mathrm{~mW}$ supplied power which is nearly the maximum power level the devices can withstand). In Figure 6.10b the normalized output of a micron-link type of heater is shown. As can be seen, upon flows of 1 to $5 \mathrm{sccm}$ the slope of the curve is low indicating low sensitivity. However, for flow/no-flow conditions are compared 

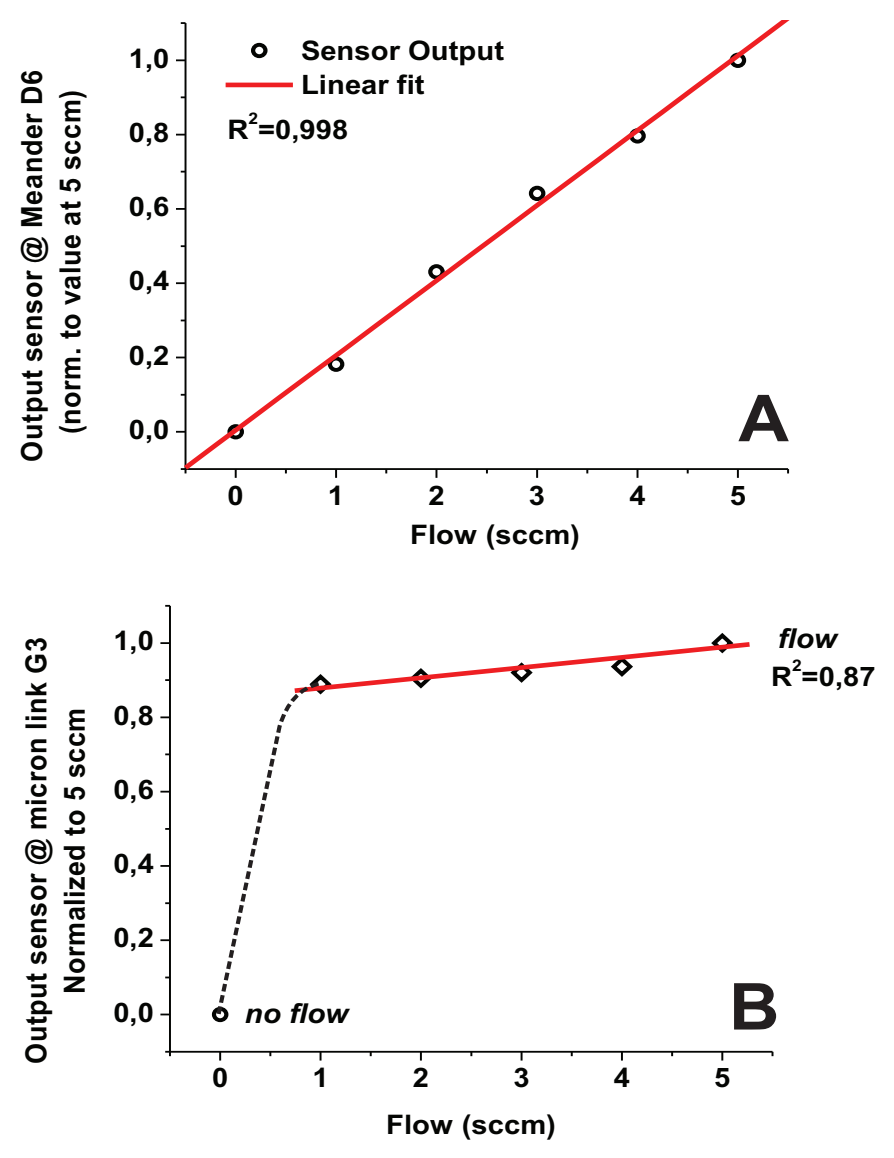

Figure 6.10: Normalized response of the flat meanderD6@w181 (a) and nano-linkG3@w181 (b) sensors to flow of nitrogen in the range $0-5 \mathrm{sccm}$.

significant differences were observed. The measurements were performed at $0.65 \mathrm{~mW}$ supplied power.

Based on the performed experiments we can conclude that the devices indeed were at elevated temperature. This is indicated by presence of the thermal losses. The rate of thermal losses from the hot element is, in this case, a useful signal and measured in response to variation in flow set point.

Responses at higher flow rates could not be measured due to limitations of device assembly and operational range of the used mass flow controllers. To enlarge the sensing flow regime, a bypass allowing larger flows shall be introduced.

\subsubsection{Thermal conductivity detector}

In Figure 6.11a the response of the flat meander heater to switching between nitrogen and hydrogen flow at various supplied power levels is shown. Clearly, a response is visible, which is the evidence that the surface of the heating element is indeed maintained at an elevated temperature. The measurements were performed at power levels between 0.3-4 mW. A micron-link heater shows a similar tendency in response to hydrogen pulsing, however, the signal is a factor of 100 less (see Figure 6.11b).

\subsubsection{Catalytic gas detector}

We did not succeed in obtaining good measurement results for the oxidation of propane over sputtered platinum catalyst for any type of heating element considered in the study. The reasons for this are: a low activity and 

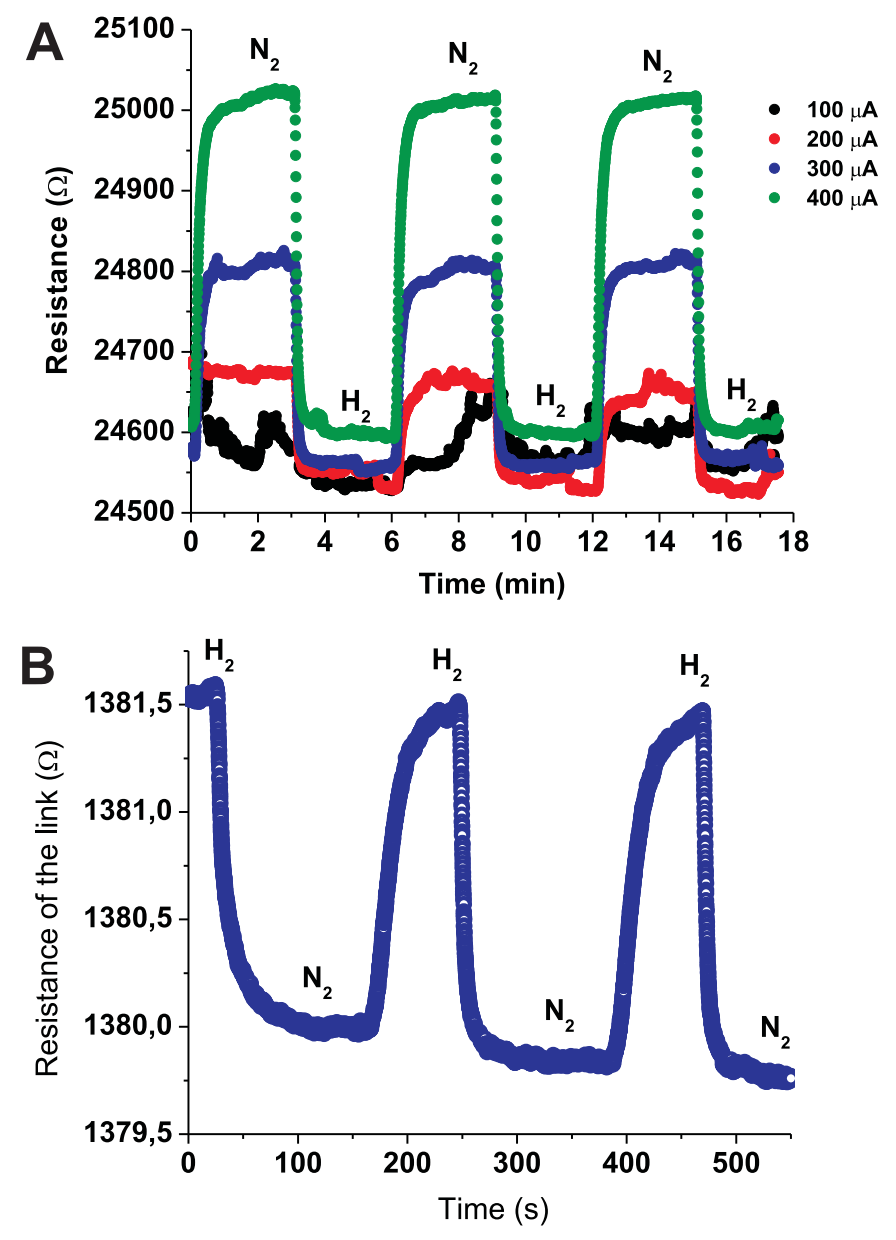

Figure 6.11: Measured resistance of the heating element as a function of time upon exposure to pulses of hydrogen and nitrogen at different measurement current 100, 200, 300, $400 \mu \mathrm{A}$ for meander type flat heater with positive TCR (a) and micron link sensor with negative TCR (b).

surface area of the sputtered Pt catalyst, low sensitivity of the heater material, noise introduced by PID, inability to deliver the required power per unit area to catalyst surface to initiate the ignition, etc.

\subsection{Outlook and remarks}

From our point of view and based on the outcome of the research conducted within SC Group [148], [149] as well as our experience with devices fabricated (run 2, w175, w181) these research directions shall be prioritized before carrying on with the gas sensitivity measurements on these devices:

- Investigation of thermo-physical properties of the resistive ALD materials and establishing the link between this knowledge and final device characteristics.

- Establishing the connection between the thermo-physical design concept, application and properties (TCR, stability at high temperature) of the resistive ALD layers comprising the device with respect to the sensor output (sensitivity). More characterization measurements should be performed on devices exhibiting a similar behavior. 
- Three applications were proposed as potentially interesting: thermal flow sensor, thermal conductivity sensor and catalytic sensor for flammable gases. The preliminary tests on number of reference flat heaters, micron- and nano- link devices have shown potential as being employed as flow sensors and thermal conductivity detectors.

- At this moment the exhibited thermal sensitivity and stability are not sufficiently high to compete with conventional micro hotplates.

- The strongest advantage of the micron- and nano- link sensor concept remains ultra-low power consumption. 


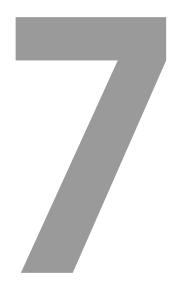

\section{Selection, characterization and local deposition of catalytic thin films.}

To ensure a specific interaction between a gas to be analyzed and a sensing interface (micro hotplate) a catalyst is immobilized. One of the most critical factors is the development of a technology for local immobilization of the catalyst (or a catalyst pattern) close to the transducer.

In this study we characterize and compare platinum (Pt) and palladium (Pd), both as sputtered thin film and as nanoparticles supported on $\gamma$-alumina catalysts, in terms of their applicability as functional coatings in catalytic gas sensors. Specifically, we discuss the deposition techniques applied, involving both self-assembly and soft-lithography, and their limitations.

${ }^{*}$ Submitted as: E.Vereshchagina, O.Bliznyuk, K.Altena-Schildkamp, R.M.Tiggelaar, J.G.E.Gardeniers, Selection, characterization and local deposition of catalytic thin films, J. Michromech. Microeng. 


\subsection{Introduction}

\subsubsection{Role of catalyst}

In catalytic microsensors used for the detection of combustible gases, e.g. methane, ethane, propane, a catalyst is an important structural element in making the response of the sensor selective for the target molecules. The measured output of the sensor is dependent on the chemical reaction occurring on the catalyst surface. The reaction is controlled by the temperature and the kinetic properties of the selected catalyst (activity, selectivity, stability, etc.). A catalyst reduces the activation energy for a reaction without being consumed and lowers the operational temperature of the sensor. Also, it fixes the location of the reaction at its surface and therewith it also fixes the location where an effect may be detected by a transducer. For this reason, local deposition of the catalyst on top of the sensing area is an important issue.

Pt and other noble metals (gold [152], palladium [153], rhodium [154]) are well established catalysts for partial and deep oxidation of hydrocarbons [155], [156], [157]. Pt and Pd are often applied as model oxidation catalysts due to their ease of use, and a large number of publications reporting kinetic data is available. The ability of $\mathrm{Pt}$ and other noble metals to lower the activation energy of many oxidation reactions makes it a useful catalyst in catalytic microsensors [158] and in catalytic converters of automobile exhaust [159], as lower temperatures are required. The main reasons for this are high activity and high resistance to over-oxidation and poisons, such as sulfur, that can cause catalyst deactivation.

Bulk noble metals have, however, poor catalytic properties. To retain a catalyst's long-term stability, highly porous catalyst supports are often implemented, which have high catalyst dispersion and, therefore, resist the sintering of small active particles to inactive metal clusters.

In this study different methods to deposit Pt- and Pd-based catalysts are applied. The catalysts were deposited as solid thin films and as nanoparticles dispersed on a highly porous alumina. The first method has the advantage of deposition and patterning in a straightforward way: by using a combination of thin metal deposition (evaporation or sputtering), traditional UV lithography and metal lift-off or wet etching. These deposition techniques are compatible with the processing of the sensor system. Post to deposition, the catalyst can be used subsequently without an activation procedure.

Supported catalysts require the development of more sophisticated deposition techniques which need to be adapted to a specific microsystem design. Some of the techniques for high-resolution, low cost patterning of ceramics from wet precursor has been reviewed by J.E. ten Elshof et al [160]. In addition, sol-gel-based catalysts can not be used for sensing directly, but need to be activated at high temperatures (above $400{ }^{\circ} \mathrm{C}$ ) prior to sensor utilization. The thermal activation procedure is not always compatible with materials comprising the sensor. The high surface area porous catalysts ensure a higher conversion and a lower sensitivity to deactivation. The response of such a system might be slower because, in order for gas to react on the surface of active metal particles, it first has to diffuse into the pores of the catalyst support. However, if the catalyst film has a thickness up to a few micrometers and contains mesopores the diffusive process is sufficiently fast, which is required for fast sensor response. From the approximated Equation 1 for diffusion time $(t)$, given the diffusion coefficient for propane $D=10^{-5} \mathrm{~m}^{2} / \mathrm{s}$ and the diffusion length equal to thickness of the porous catalyst film $l_{d i f}=1 \mu \mathrm{m}$, times needed for propane to diffuse are in order of $100 \mathrm{~ns}$ :

$$
t \sim \frac{l_{d i f}^{2}}{4 D}
$$

\subsubsection{Specifications of the catalyst and catalyst deposition}

The choice of a catalyst is application specific and determined by the combination of catalyst and feasibility of techniques for local deposition and patterning. Our application is the detection of combustible gases in air. The following factors are considered the most important:

- High activity as oxidation catalyst;

- Resistance to over-oxidation, poisoning and sintering; 
- Compatibility of the catalyst activation procedure with the materials of the sensor;

- Applicability of the deposition method at the microscale for local deposition and patterning (a catalyst pattern distributes mechanical stress induced at high temperature).

The activity of Pt has an impact on the heat generated and the operating temperature of the sensor. Although Pt is a good catalyst for a wide range of hydrocarbons, some degree of selectivity can be achieved based on two measurables: ignition temperature and heat release [161].

$\mathrm{Pt}$ is known for being resistant to deactivation at high temperatures. This gives a long lifetime and stability of the sensor when operated in an aggressive and (or) oxidizing environment. Pd is an alternative catalyst, but is more prone to oxidation.

Often, prior to utilization, the catalysts need to be activated which is achieved by heat treatment (calcination, reduction at temperatures above $300{ }^{\circ} \mathrm{C}$ ) in a hydrogen containing ambient. This high temperature annealing step has to be compatible with the materials comprising the sensor.

Technology must ensure proper operation of both the sensor and the catalyst afterwards, and prioritize local positioning of the catalyst pattern in a reproducible manner.

\subsubsection{Deposition techniques}

To ensure a specific interaction between the gas analyzed and a sensing interface (micro hotplate) a catalyst is immobilized. One of the most critical factors is the development of a technology for local immobilization of the catalyst (or catalyst pattern) close to the transducer.

Deposition of thin catalyst films can be achieved using evaporation or magnetron sputtering. There is, however, no standardized approach for immobilization of sol-gel based catalytic materials. The development of techniques enabling integration of sol-gel catalysts is important as they are commonly used as functional coatings in micromachined gas sensors [162] and catalytic microreactors [163].

Although there exist a wide range of techniques for deposition on structured surfaces [164] only few are compatible with processing on a micro scale and give satisfactory results in terms of reproducibility. Due to these limitations, mostly basic techniques such as drop- or spin-coating have been adapted to deposit the catalysts on micro hotplates [165]. These, however, have drawbacks such as poor adhesion and low yield of functioning devices.

Here we propose to use soft lithographic techniques for deposition and patterning of the boehmite $(\mathrm{AlO}(\mathrm{OH}))$ sol used as a support precursor for $\gamma-\mathrm{Al}_{2} \mathrm{O}_{3}$.

Soft lithography is a low cost technology for the direct deposition and patterning of colloidal suspensions [166]. It was introduced by G. Whitesides as an alternative technology to standard photolithography allowing microfabrication of high-resolution structures in laboratory conditions [167]. Traditionally, flexible polydimethylsiloxane (PDMS) molds, prepared by casting in a patterned master, are used for transferring the microstructures. The master is prepared by any other lithographic technique which defines the smallest topological feature required. There are several techniques which enable the transfer of a pattern with PDMS molds [168]: microcontact printing ( $\mu \mathrm{CP}$ ) [169], micromolding in capillaries (MIMIC) [170], microtransfer molding $(\mu \mathrm{TM})$ and minor modifications of these. The techniques used in this study are schematically demonstrated in Figure 7.1. In MIMIC a PDMS mold, containing channels opened from one side, is placed on the surface upside down. When a droplet of the solution to be patterned is applied on one side of the open channels, capillary forces cause spontaneous filling [172]. This technique was successfully applied for the deposition of metal oxides in thick film gas sensors [173], [174].

In $\mu \mathrm{TM}$ a material to be patterned is spread over the PDMS mold and placed on the substrate. After temperatureassisted curing the mold is removed.

Criteria used for comparison of the selected catalyst deposition techniques are listed below:

1. Feasibility of local deposition;

2. Applicability on microscale and compatibility with Si-based sensors; 
3. Smallest possible dimension;

4. Simplicity of the method;

5. Reproducibility of the method;

6. Cost efficiency;

7. Generic deposition approach for various materials;

8. Feasibility of wafer scale processing.

In $\mu \mathrm{CP}$ a PDMS mold is used to transfer a pattern of "ink" to a substrate through conformal contact with the formation of a covalently attached self-assembled monolayer (SAM) [171].

\subsection{Experimental}

\subsubsection{Catalyst preparation}

Based on the specifications mentioned in section 1.2 two catalysts were selected as model system for the catalytic gas sensor: (a) solid thin films of Pt and Pd on Ta and (b) Pt and Pd nanoparticles immobilized on porous $\gamma-\mathrm{Al}_{2} \mathrm{O}_{3}$.

\section{Sputtered thin film catalyst}

Pt and Pd thin films with a thickness of $100 \mathrm{~nm}$ were deposited at a pressure of $0.066 \mathrm{mbar}$, in $148 \mathrm{sccm}$ Ar flow and a power of $200 \mathrm{~W}$ using a custom-built DC magnetron sputtering system. Ta was used as an adhesion promoter as it was reported to be stable and resistant in a high temperature oxidizing environment [104]. Patterning was done using conventional UV lithography and thin film lift-off (30 min sonication in acetone). Shown in Figure 7.2 is a High-Resolution Scanning Electro Microscopy (HRSEM) image of a patterned Pt (100 nm)/Ta $(15 \mathrm{~nm})$ thin film formed by a lift-off method. Due to a slight coverage of the resist sidewalls by sputtered metal, so called "ears" were formed, visible on the image. The pattern of squares was used to distribute the heat homogeneously and reduce the impact of mechanical stress on both catalyst and catalyst support.

\section{Pt and Pd on sol-gel-based catalyst}

Pt and Pd on $\gamma$-alumina $\left(\gamma-\mathrm{Al}_{2} \mathrm{O}_{3}\right)$ catalyst were prepared in four steps:

1. Preparation of the boehmite $(\mathrm{AlO}(\mathrm{OH}))$ sol, used as a precursor for the $\gamma-\mathrm{Al}_{2} \mathrm{O}_{3}$, via the colloidal sol gel route;

2. Impregnation of the boehmite sol with the Pt and Pd precursors;

3. Calcination in air at $400{ }^{\circ} \mathrm{C}$ to form porous $\gamma-\mathrm{Al}_{2} \mathrm{O}_{3}$ and decompose the noble metal precursor (PtO, $\left.\mathrm{PdO}_{2}\right)$

4. Reduction in $\mathrm{H}_{2} / \mathrm{N}_{2}(10 / 90)$ at $400{ }^{\circ} \mathrm{C}$ to reduce dispersed metal particles ( $\left.\mathrm{PtO}, \mathrm{PdO}_{2} \rightarrow \mathrm{Pt}, \mathrm{Pd}\right)$.

The sol-gel method used for preparation of the boehmite sol was developed earlier [175] and optimized by Biesheuvel et al. [176] to achieve highly porous, thermally-resistant, low-stress $\gamma-\mathrm{Al}_{2} \mathrm{O}_{3}$ membranes.

The calcination and reduction temperature profile is shown in Figure 7.3. A temperature of $400{ }^{\circ} \mathrm{C}$ and a duration of 10 hrs was sufficient for the transformation to a stable $\gamma$-phase of $\mathrm{Al}_{2} \mathrm{O}_{3}$. 

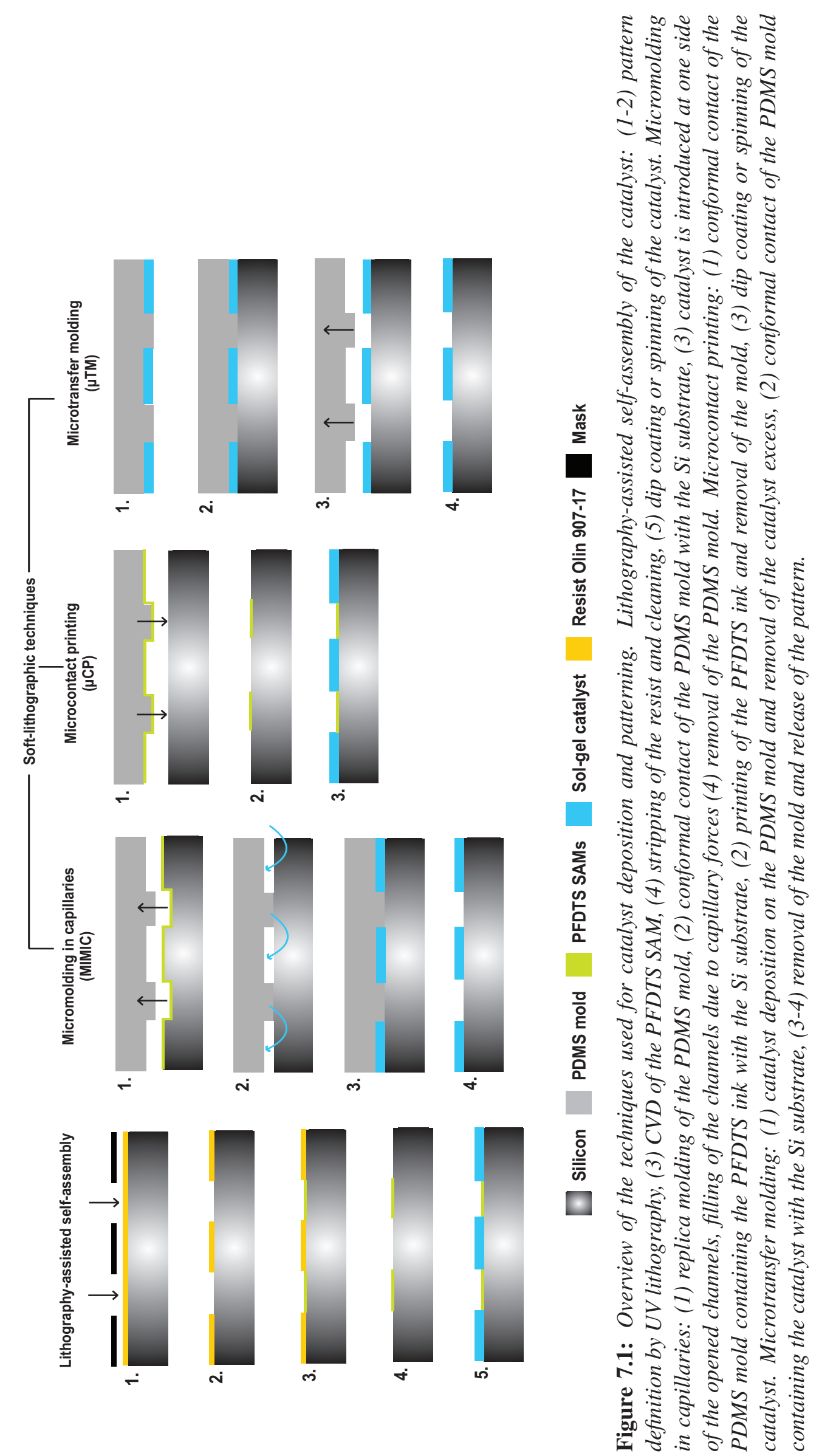


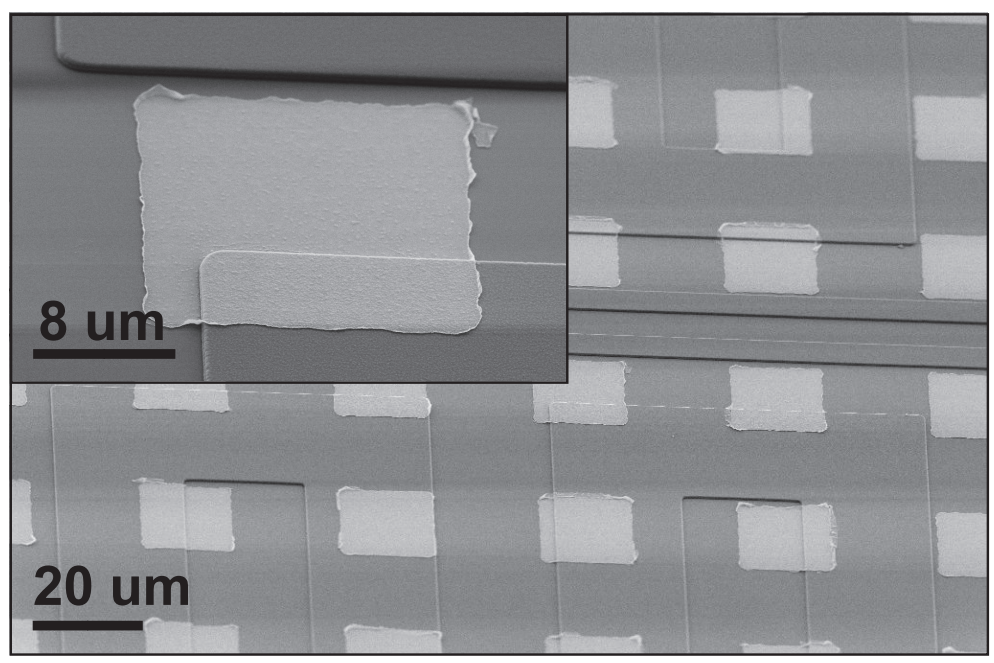

Figure 7.2: HRSEM image of the Pt/Ta thin film catalyst sputtered and patterned with the lift-off process on the micro hotplate surface. The magnified image shows the "ears" mentioned in the text, i.e. small protrusions at the edge of the metal pattern.

$\mathrm{Pt} / \gamma-\mathrm{Al}_{2} \mathrm{O}_{3}$ and $\mathrm{Pd} / \gamma-\mathrm{Al}_{2} \mathrm{O}_{3}$ catalysts were prepared by wet impregnation of the commercial chloride and nitrate precursors. For $\mathrm{Pt}$ hexachloroplatinic acid solution $\left(\mathrm{H}_{2} \mathrm{PtCl}_{6}, 20 \mathrm{wt}\right.$. \%) and tetraammineplatinum nitrate $\left(\mathrm{Pt}\left(\mathrm{NH}_{3}\right)_{4}\left(\mathrm{NO}_{3}\right)_{2}, 4 \mathrm{wt} . \%\right)$ were used. The precursors used for the preparation of $\mathrm{Pd} / \gamma-\mathrm{Al}_{2} \mathrm{O}_{3}$ catalyst were palladium (II) chloride $\left(\mathrm{PdCl}_{2}, 20\right.$ wt. \%) and palladium (II) nitrate $\left(\mathrm{Pd}\left(\mathrm{NO}_{3}\right)_{2}\right.$, wt. 14-16 \%).

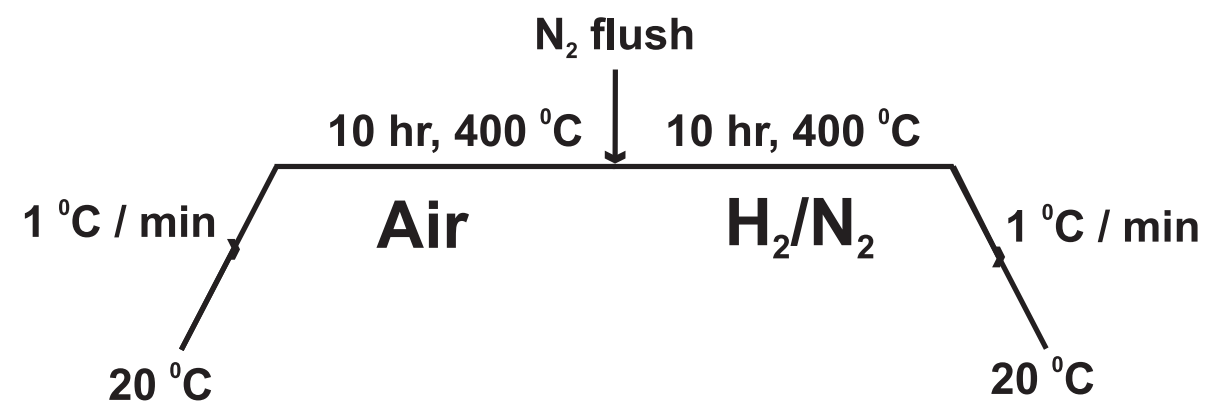

Figure 7.3: The heat treatment cycle used for the formation of the $\gamma-\mathrm{Al}_{2} \mathrm{O}_{3}$ and activation of the metal catalyst.

\subsubsection{Soft-lithography}

\section{Preparation of the mold}

PDMS was prepared by cross linking of Si-based organic polymer Sylgard 184 (18500 D) with a hydroxy silane cross linker (684 D).

The PDMS molds were made by replica molding from a microstructured silicon master, followed by heat treatment at $80{ }^{\circ} \mathrm{C}$ for two hours. The curing rate of the PDMS molds is dependent on temperature. The ratio of polymer and cross-linker in combination with temperature are parameters that influence the mechanical durability of the molds.

PDMS affinity towards Si and glass can be modified by treatment in an oxygen plasma.

Two types of masters for replica molding were utilized:

- Grooves formed by patterning of a layer of SU-8 resist coated on a Si substrate;

- Grooves formed by Reactive Ion Etching (RIE) of a Si substrate through an Olin-1.7 resist mask.

Examples of the masters are shown in Figure 7.4. 

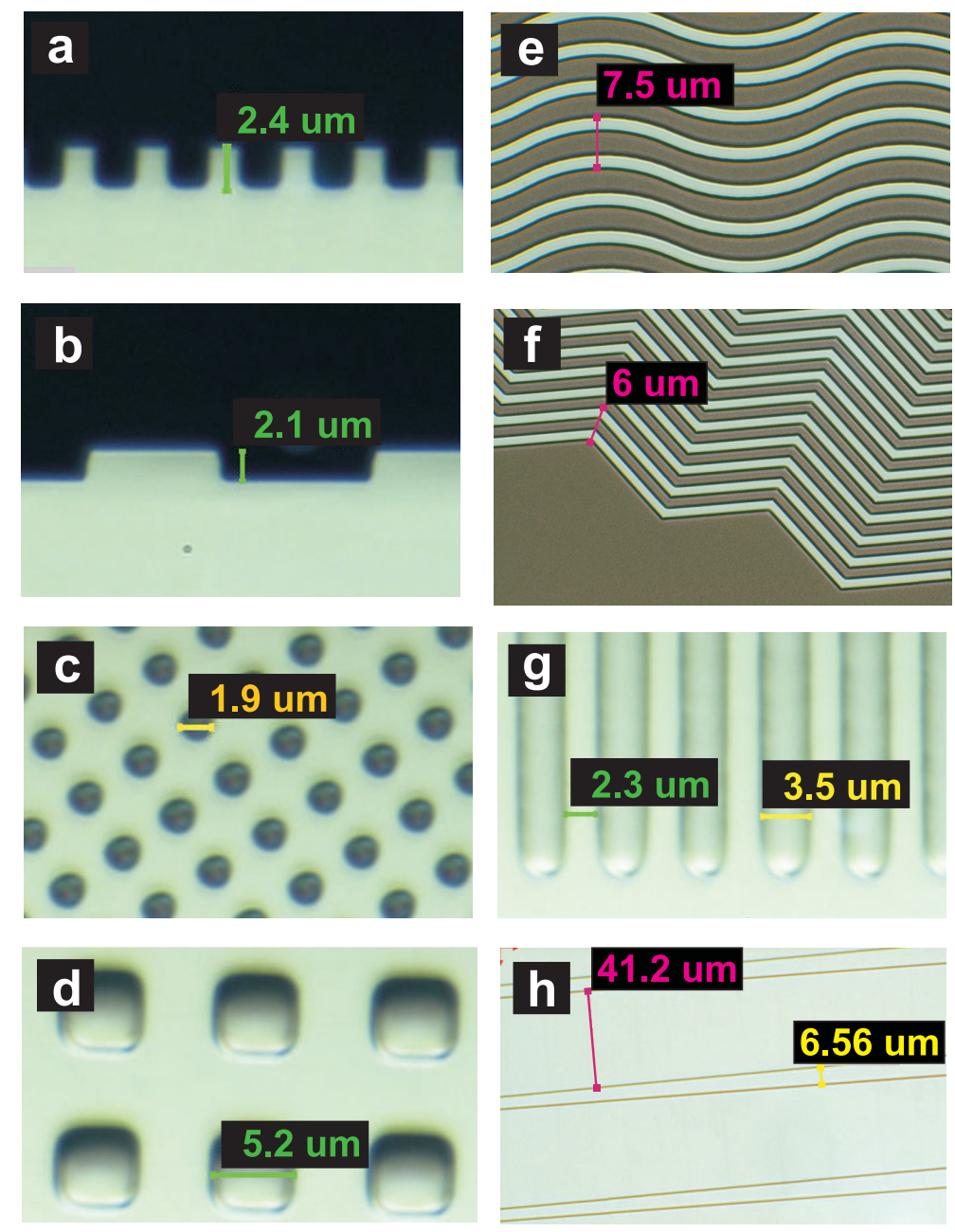

Figure 7.4: Various designs of the master used as a template for the PDMS mold replication: (a-b) optical imaging of the cross section showing not completely vertical wall profile which corresponds to the effect of underetch from RIE; (c) distortion of the square shape pattern due to lithgraphic limitations; $(d-h)$ stripe and square patterns used as a mask for the surface modification or replicated for direct catalyst patterning with the PDMS mold.

\section{Pattern transfer}

The following techniques were used for deposition and patterning (see Figure 6.1): direct deposition and patterning of boehmite sol using MIMIC and $\mu \mathrm{TM}$, chemical patterning (using $\mu \mathrm{CP}$ of functional groups and conventional lithography in combination with Chemical Vapor Deposition (CVD) of functional groups) with the subsequent deposition of the boehmite sol dropwise using piezoactuated droplet dispensing, and using spinand dip- coating.

The procedure used for patterning using the MIMIC method consists of the following steps:

1. The PDMS mold was placed on the substrate ensuring a good conformal contact;

2. The liquid catalyst precursor was placed at the entrance of the open mold pattern;

3. Filling of the pattern occured due to capillary forces;

4. The mold was removed;

5. Temperature treatment of the patterned catalyst is performed as described in section 6.2.1. 
In $\mu \mathrm{TM}$ the PDMS mold was first immersed in the catalyst solution, followed by the removal of the material excess, and then directly transferred onto the substrate. This was followed by curing at ca. $100{ }^{\circ} \mathrm{C}$ and release of the mold. The curing temperature was selected to be sufficient for evaporation of the water from the catalyst precursor, but below the maximum permitted temperature for PDMS (ca. $150{ }^{\circ} \mathrm{C}$ ).

$\mu \mathrm{CP}$ and lithography-assisted catalyst self-asssembly included one more step prior to immobilization of the catalyst, i.e. chemical surface modification of the oxidized silicon surface using the PFDTS SAMs $(1 \mathrm{H}, 1 \mathrm{H}$, $2 \mathrm{H}, 2 \mathrm{H}$, perfluorodecyltrichlorosilane).

Oxidized $\mathrm{Si}$ is a high energy solid surface with a high critical surface tension $(150 \mathrm{~mJ} / \mathrm{m})$, and therefore wetted by the majority of liquids. The hydrophilicity of the Si surface can be modified with a hydrophobic molecular layer of the type - $\left(\mathrm{CH}_{2}\right)$ - or - $\left(\mathrm{CF}_{2}\right)$-. For hydrogenated and fluorinated coatings the surface tension drops (20 $\mathrm{mJ} / \mathrm{m}$ ) and these surfaces exhibit highly non-wetting behavior [177].

To introduce a chemical pattern on a Si surface we used SAMs of PFDTS. The PFDTS molecule has a fluorinated chain consisting of 10 carbon atoms and a silane head-group that binds covalently to a thin layer of native oxide present on the $\mathrm{Si}$ wafer. Once oxidized silicon chemically reacts with PFDTS a fluorinated tail is exposed. The presence of fluorinated groups results in overall hydrophobic behavior of the self-assembled thin film. The thin films formed are densely packed with a height, measured by AFM, ca. $0.8 \mathrm{~nm}$.

The proposed method of pattern transfer is based on CVD of PFDTS [178]. The procedure was developed by H. Rathgen et al. [179]. Deposition was carried out in a degased chamber. The silicon master containing a resist pattern made by UV lithography was exposed to PFDTS vapor. Afterwards the reservoir containing PFDTS was closed and water vapor was introduced to initiate the reaction. By adjusting the pressure the amount of water and PFDTS vapor entering the chamber can be regulated, thus, leading to controlled and reproducible deposition of SAMs. The Si wafer contains a layer of resist with defined openings which were exposed to PFDTS. The PFDTS vapor react with the oxygen atoms on $\mathrm{Si}$ wafer to the formation of covalent bonds. PFDTS does not react with the photoresist. After the reaction was carried out the resist was removed in acetone and the wafer was cleaned by immersion in concentrated nitric acid.

\subsection{Results and Discussion}

\subsubsection{Sputtered thin films}

The sputtered thin films have a preferential orientation $\{111\}$ which was reported for identical sputtered thin films by R. Tiggelaar et al. [104]. This is an unfavorable orientation when used as a catalyst. It has been reported that catalyst with densely packed atoms (such as $\{111\}$ or $\{100\}$ ) exhibit low catalytic activity. Conversely, a catalyst characterized by high index planes provides more active sites where reactant molecules can interact [180].

The number of active catalyst centers on the surface of thin platinum catalyst was estimated using chemisorption with $1 \%$ carbon monoxide (CO) 4.7 as a pulsing gas. The experiment confirmed a low surface area of active sites, ca. $10^{-4} \mathrm{~m}^{2} / \mathrm{g}$, see Table 1 . To increase the accessibility to gas a square pattern was introduced as described earlier (see section 6.2.1), with the resolution limited by UV lithography to $2 \mu \mathrm{m}$.

The surface area does not change dramatically when patterned. However, the walls and edges of the pattern can be more catalytically active than the top layer. Implementation of the pattern results in a discontinuity of the catalyst, i.e. defects acting as reactive centers. An alternative approach would be to roughen the surface of the sputtered thin film or realize a channel profile in Pt such that the gas can react at both the channel bottom and on the side walls. This would also lead to more efficient storage and distribution of the heat.

\subsubsection{Porous thin films}

Presence of the desired $\gamma-\mathrm{Al}_{2} \mathrm{O}_{3}$ was verified by X-ray Diffraction Analysis. Shown in Figure 7.5 are the diffractograms taken from the powder samples of uncalcined boehmite sol and $\gamma$-alumina. The peaks identified confirmed the formation of $\gamma$-alumina. Thermal treatment may cause crack formation and (or) detachment of 
the catalyst from the substrate (see Figure 7.6). Therefore, $1{ }^{\circ} \mathrm{C} / \mathrm{min}$ heating and cooling rates were used (to reduce mechanical stresses due to the high temperature). $\gamma-\mathrm{Al}_{2} \mathrm{O}_{3}$ was selected as a support due to the high

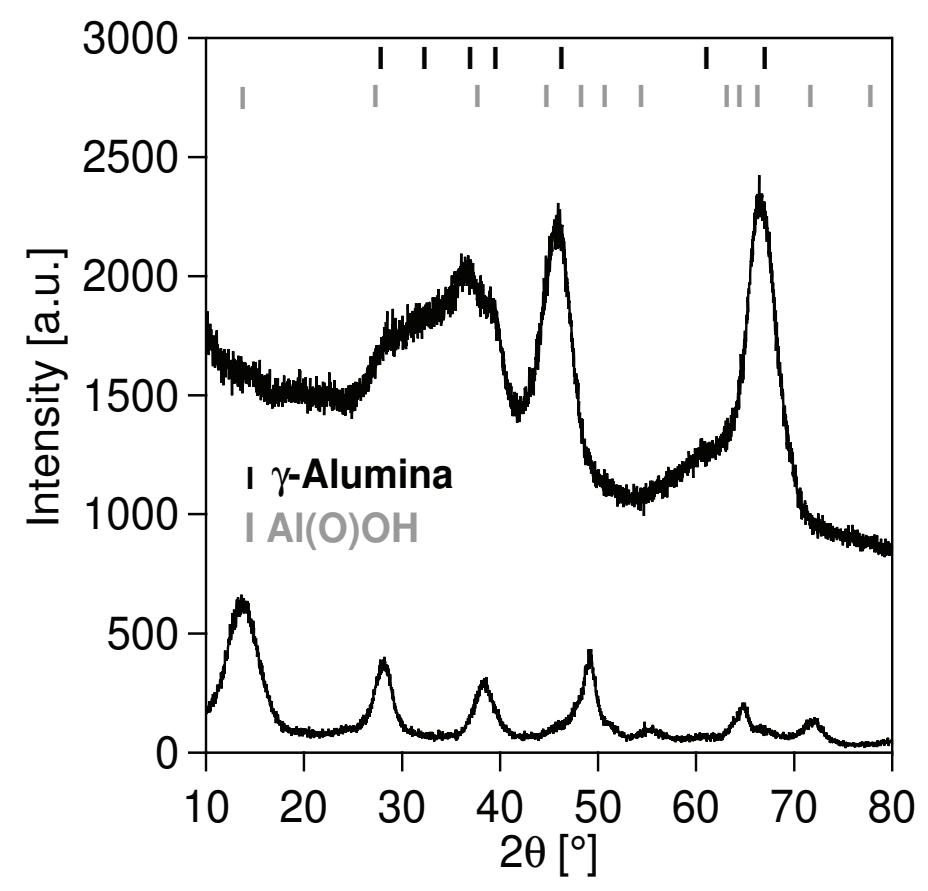

Figure 7.5: The $X$-ray diffractograms taken after preparation of the boehmite sol and calcination resulting in $\gamma$-alumina.

surface area. The surface area was estimated using the BET method and was found to be $383.5 \mathrm{~m}^{2} / \mathrm{g}$.

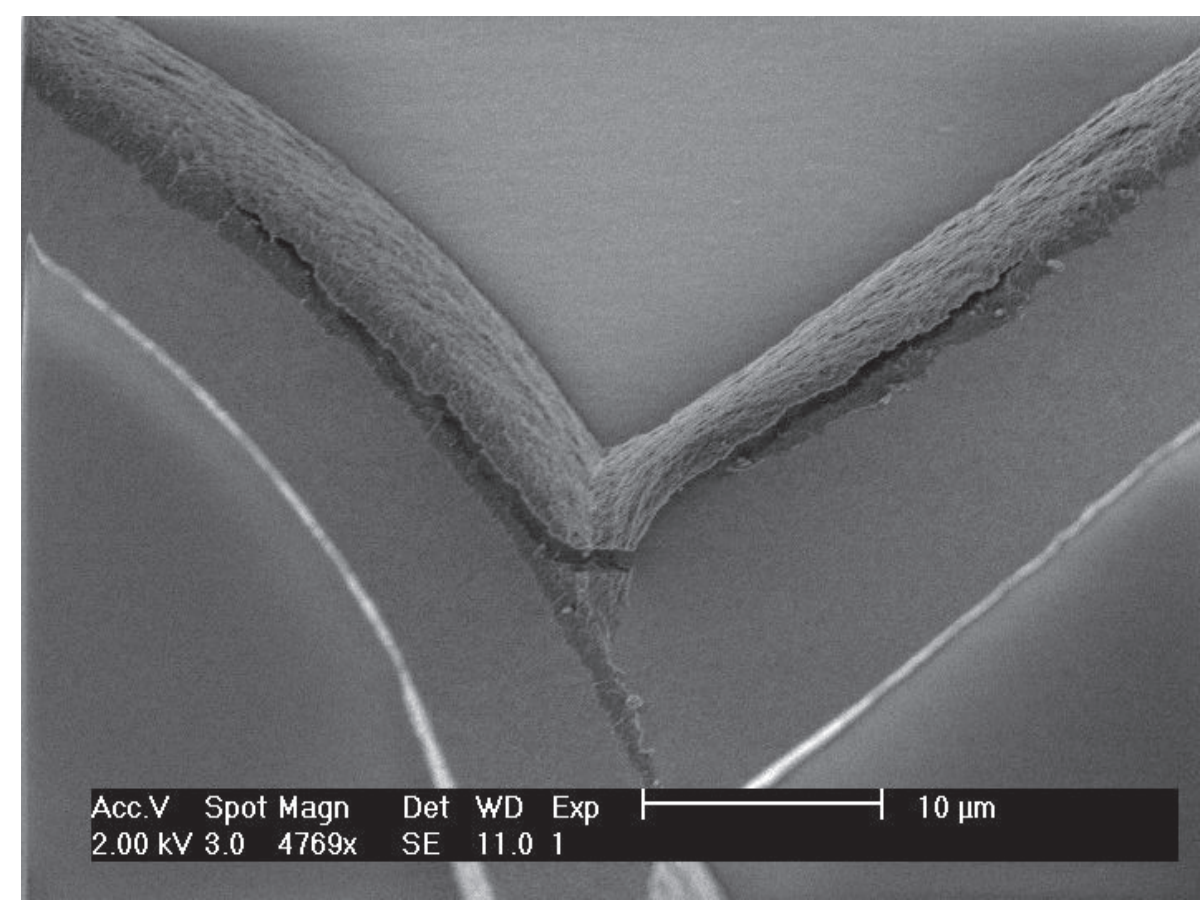

Figure 7.6: Stress induced detachment of the catalyst from the substrate. 


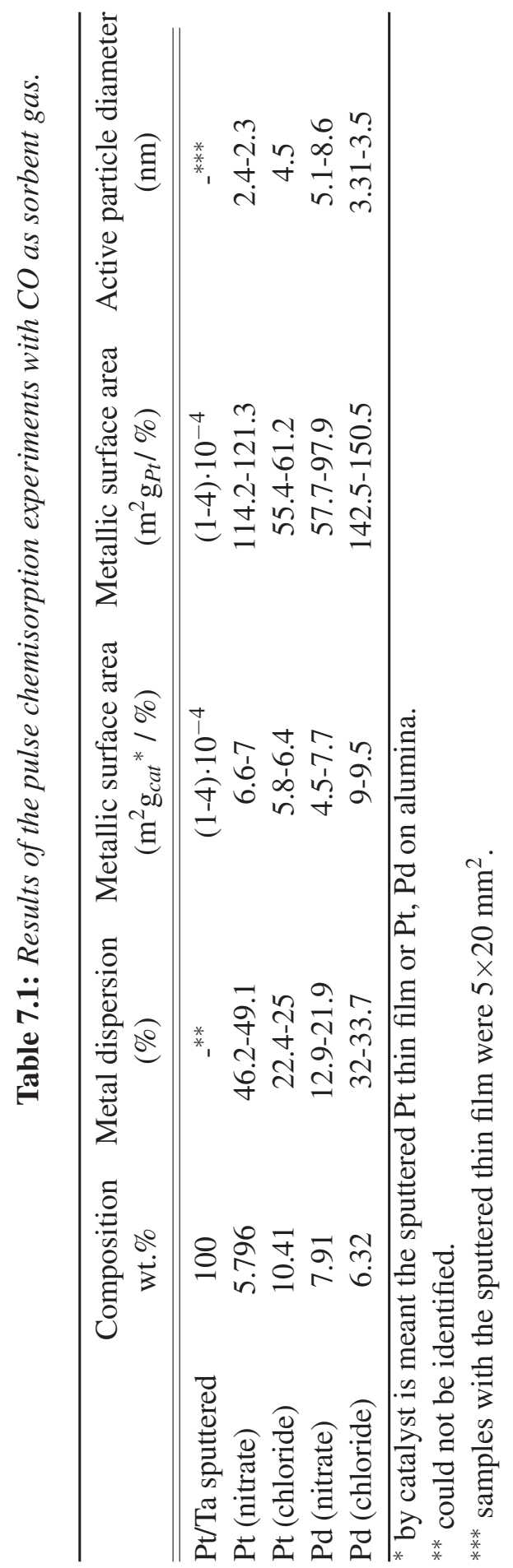


Pt is well dispersed on $\gamma-\mathrm{Al}_{2} \mathrm{O}_{3}$ which can be seen from Transmission Electron Microscopy analysis (see Figure 7.7) of the powder catalysts prepared. The average particle size is ca. 5-6 nm. The smaller the particle size, the larger the amount of low coordinated Pt atoms (defects, edges) that act as reactive centers, thus the higher the activity of the catalyst per gram. Catalysts containing smaller particles are more prone to oxidation and sintering, i.e. faster deactivation, but on the other hand, lead to high metal dispersion. It was found that
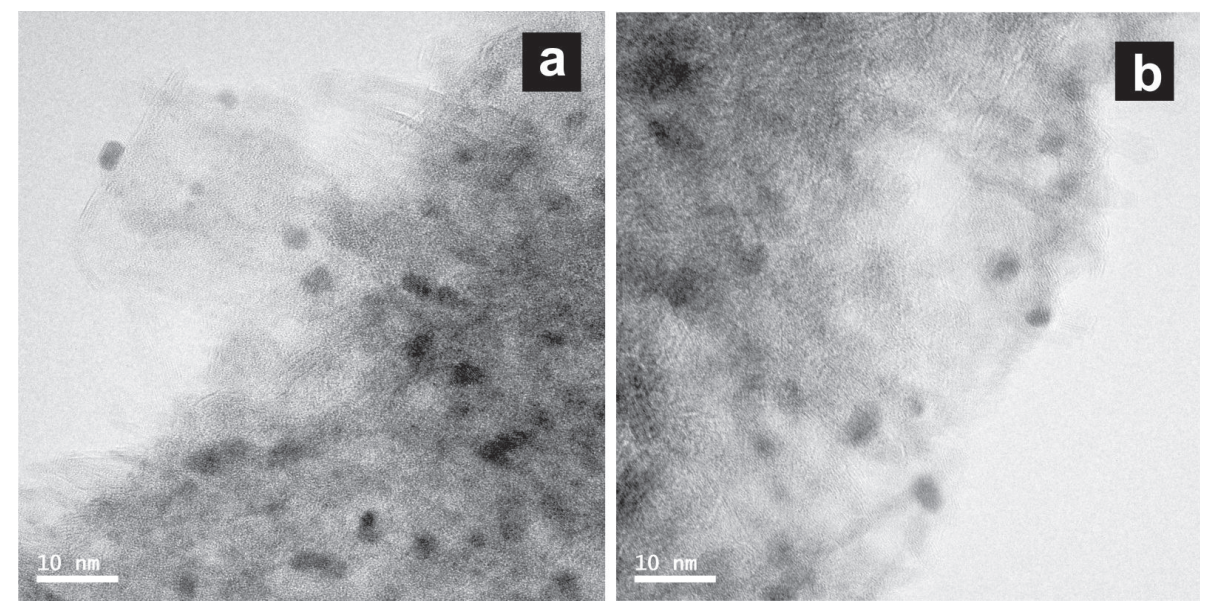

Figure 7.7: Transmission electron microscopy image of the $\mathrm{Pt} / \gamma-\mathrm{Al}_{2} \mathrm{O}_{3} / 5 \mathrm{wt} . \%$ impregnated from the chloride solution $\left(\mathrm{H}_{2} \mathrm{PtCl}_{6}\right)(a), \mathrm{Pd} / \gamma-\mathrm{Al}_{2} \mathrm{O}_{3} / 5$ wt. \% impregnated from the nitrate solution $\left(\mathrm{Pd}\left(\mathrm{NO}_{3}\right)_{2}\right)(b)$ showing welldispersed catalysts particles with an average size of 5-6 $\mathrm{nm}$.

sols containing a chloride precursor were highly aggressive for the material of the sensor, in particularly to the $\mathrm{Al}$ metallization layer. This was attributed to formation of hydrochloric acid $(\mathrm{HCl})$ during the calcination. Chloride ions, originating from the precursor, were also reported to inhibit catalytic activity [181]. They can be partly removed by high temperature treatment which is not compatible with the CMOS-based sensor materials. Pulsed CO chemisorption was used for the determination of the active Pt and Pd surface, the size of active catalyst particle and metal dispersion. The results of chemisorption experiments are given in Table 1. The metallic surface area is several orders of magnitude higher for the porous catalysts compared to the thin sputtered catalyst.

\subsubsection{Choice of the catalyst}

Surface area, dispersion and activity of a metal phase are interrelated parameters which dictate the choice of catalyst.

Sputtered thin films are characterized by a low surface area and, if there is a non-active phase present on the top layer, low activity. However, introducing a pattern may resolve this issue. The catalyst does not require any specific activation procedure after deposition and can be used directly.

Sol-gel based supports ensure a high surface area and a high dispersion, resulting in high activity. However, a procedure for the selection of a suitable catalyst precursor and activation procedure is required. Moreover, the catalyst can not be used subsequently and needs to be activated.

\subsubsection{Deposition on chemically patterned $\mathrm{Si}$}

The surface of oxidized silicon was chemically modified using the PFDTS SAMs as discussed in section 6.2.2. The motivation for this is to introduce areas with different surface energies, i.e. with hydrophobic or hydrophilic (non-wetting or wetting) behavior. When applied on top of such surface the catalyst "feels" the pattern and shows a different affinity towards patterned and non patterned surfaces: repelled from the hydrophobic areas and adhering on hydrophilic sections. As a result, deposition can be achieved selectively and locally: on the area of interest. This technique allows controlled deposition and patterning of the catalyst in a non-destructive 
manner, for both the sensor and the catalyst, with a resolution limited only by the mask fabrication method. The wetting behavior of the boehmite sol on a patterned Si surface was investigated. Boehmite sol, used as
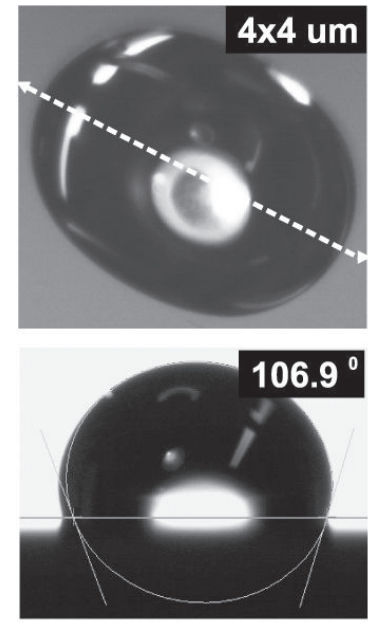
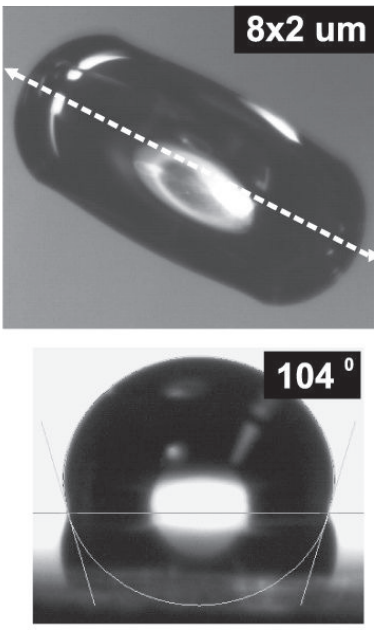
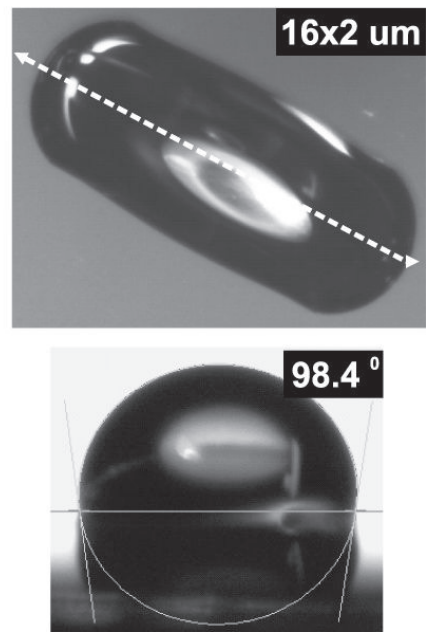

Figure 7.8: Droplets of the boehmite sol deposited on the chemically patterned surface (white line shows direction of the line pattern) with various ratio of hydrophilic and hydrophobic areas; corresponding contact angles are shown below each photograph.

a precursor for $\gamma-\mathrm{Al}_{2} \mathrm{O}_{3}$, has viscosity and wettability properties close to those of pure water. The viscosity of boehmite was measured at room temperature and found to be equal ca. $3.5 \mathrm{mPa} \cdot \mathrm{s}$ (for comparison the viscosity of water is $1.002 \mathrm{mPa} \cdot \mathrm{s})$. This is an important property for the material to be patterned by various soft lithographic techniques. The boehmite droplets (volume of dispensed droplets kept constantly $3 \mu 1$ ) deposited on the stripe-patterned surfaces are demonstrated in Figure 7.8. The stripe pattern was realized with various widths of hydrophobic and hydrophilic areas. Symmetrical patterns, such as $4 \times 4 \mu \mathrm{m}$, result in a (nearly) round shape of the boehmite droplets.

When the width of the hydrophilic part was increased compared to chemically modified hydrophobic areas, such as from $8 \times 2 \mu \mathrm{m}$ to $16 \times 2 \mu \mathrm{m}$, elongation of the droplets was observed. Similar behavior was observed with water and glycerol with mechanism for this explained in [182], [183].

The difference in the affinity of the boehmite sol to hydrophobic and hydrophilic areas causes a deformation of the droplet and preferential orientation along the lines. Thus, by creating a specific pattern, manipulation with a limited amount of the catalyst precursor is possible. When the volume deposited becomes too large the macroscopic effects play a more important role and the boehmite sol does not respond anymore to the hydrophobic/hydrophilic pattern underneath the droplet volume. Contact angle measurements of boehmite and water droplets on equally patterned surfaces indicated nearly the same angles (ca. $100^{\circ}$ ), from which we can conclude that the wetting behavior of boehmite is similar to that of water. Spin coating and dip coating can be used to deposit the boehmite on a chemically patterned surface. Figure 7.9 and 7.10 show examples of the self-assembled boehmite for wide lines and squares.

It was possible to achieve stable adhesion of the catalyst, which was tested by sonification in water. After 20 min of ultra-sound treatment no mass loss was observed.

Formation of the PFDTS SAM chemical pattern on Si can also be achieved by the $\mu \mathrm{CP}$ method. Although, it was reported as being successfully applied in patterning of colloidal suspensions [184], in our case this method is less promising. Silanes are known for being unreactive when an insufficient amount of water is present. On the other hand, in an excess of water they tend to polymerize. Therefore, deposition has to be carried out in a controlled environment, e.g. a glove box, and even then humidity control is crucial. The polymerization reaction requires a defined amount of time, thus when directly exposed to air the printed ink still keeps its properties for a few minutes, but this reduces the reproducibility of the patterned films. Moreover, the $\mu \mathrm{CP}$ technique in most of the cases requires transfer of the pattern chip by chip. Therefore, the procedure of surface 


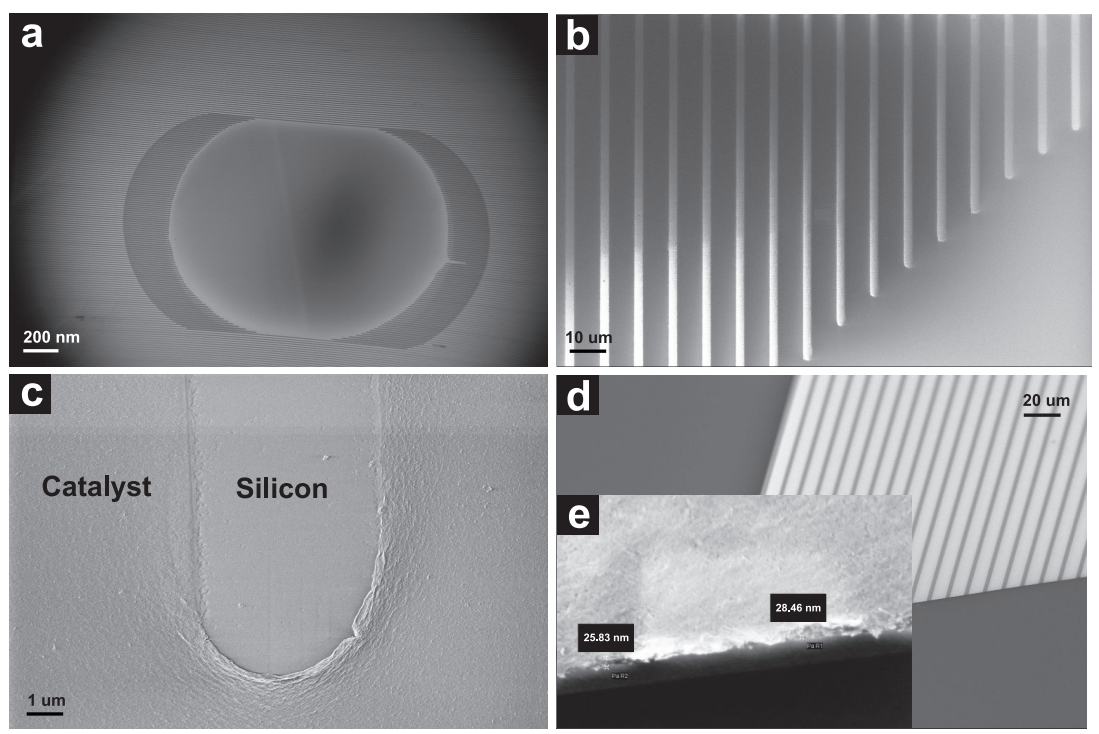

Figure 7.9: HRSEM images of the the boehmite patterns obtained by droplet wise deposition (a), pinning effect at the edges of the deposited droplet (b), self-assembly of the boehmite on a non-patterned silicon surface and free of catalyst chemically modified surface $(c)$, cross sectional view on patterned structures $(d)$ showing average thickness in order of 25-30 $\mathrm{nm}$ (e).

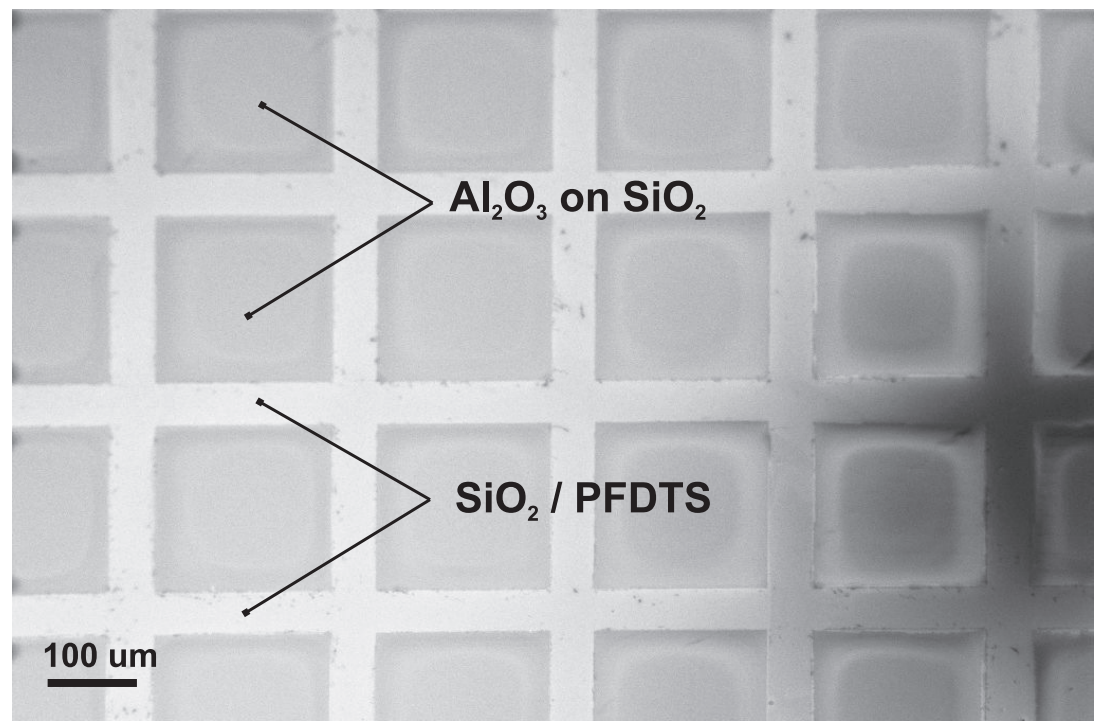

Figure 7.10: Square pattern of catalyst thin film obtained by dip coating of chemically patterned $\mathrm{Si}_{\mathrm{SiO}}$ substrate in the boehmite solution. Low energy (hydrophobic) areas with immobilized PFDTS SAM repel the boehmite, high energy (hydrophilic) $\mathrm{SiO}_{2}$ surface facilitates deposition of the boehmite.

modification by $\mu \mathrm{CP}$ alone is a time-inefficient process with a low rate of success.

\subsubsection{Direct deposition using the PDMS mold}

The MIMIC technique was utilized for patterning of boehmite sol on microstructured surfaces. In Figure 7.11 an example of the pattern achieved by MIMIC is demonstrated. Structures with a resolution down to $4 \mu \mathrm{m}$ were achieved. This method can be adopted for the patterning of various liquid catalyst precursors. However, in each case an experimental procedure needs to be developed at which the affinity of the material to be patterned is higher towards the solid surface than the mold, to prevent sticking. 

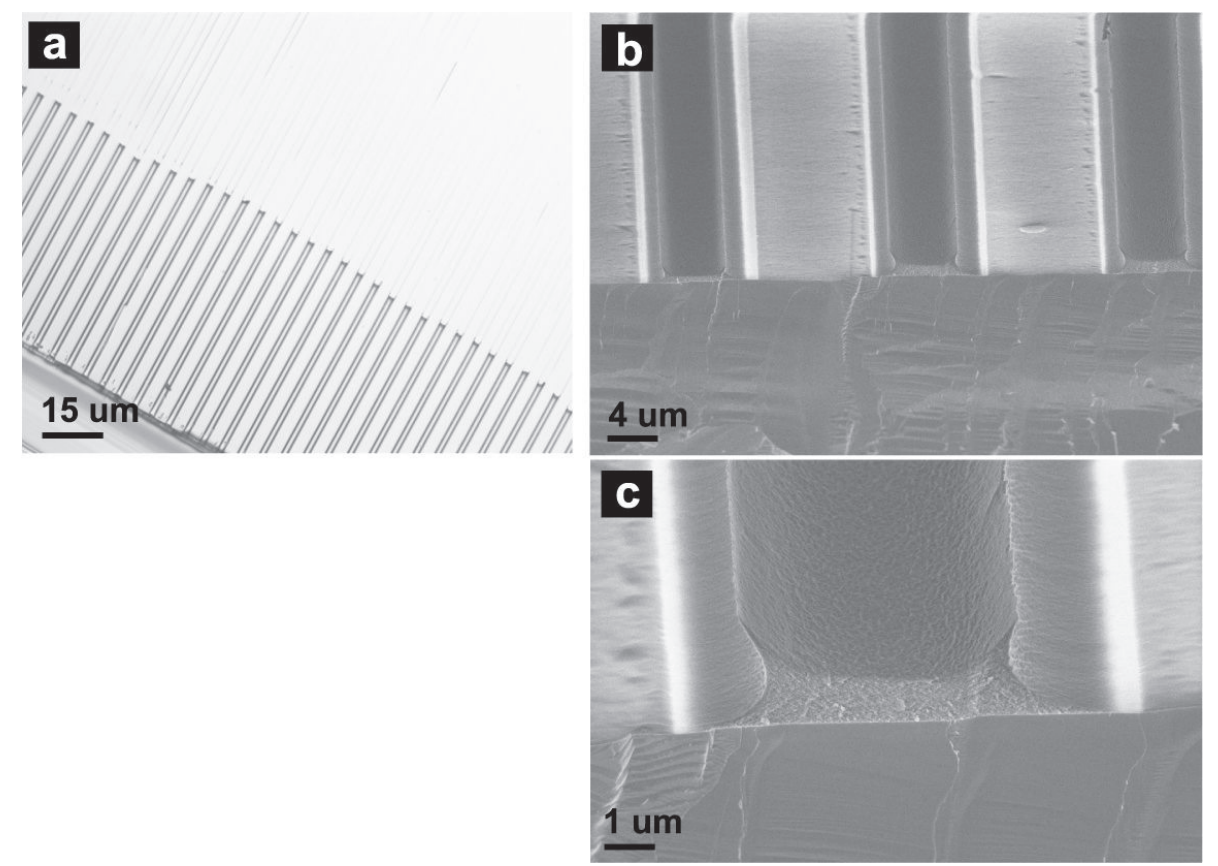

Figure 7.11: Instantaneous filling of the PDMS channels due to capillary forces (a), the $\gamma-\mathrm{Al}_{2} \mathrm{O}_{3}$ pattern obtained by the MIMIC technique (b), close up showing sintering of the oxide during the calcination $(c)$.

Additionally, after calcination the $\gamma-\mathrm{Al}_{2} \mathrm{O}_{3}$ tends to shrink, causing deformation of the original pattern. This factor has to be considered in the original design.

In $\mu \mathrm{TM}$ the catalytic precursor is transferred by the mold. After placing the mold containing the precursor on the substrate, a temperature treatment is applied to dry the catalytic precursor. The main factor limiting implementation of this technique is the time for adjusting the affinity of the material to the mold and substrate in combination with the temperature treatment step. These factors cause sticking of the catalytic material to walls of the PDMS molds. In addition, initial removal of the catalyst excess results in non-clean conformal contact with the substrate. Due to these reasons the method is regarded as less efficient for the purpose of the sol-gel catalyst deposition.

\subsubsection{Choice of the deposition technique}

In this study we aimed to select a technique which can be directly applied for the deposition of sol-gel based catalysts; which does not require a long tuning procedure for the material to be patterned and gives reproducible results.

The best results in terms of reproducibility and time efficiency were achieved by lithography-assisted selfassembly of the catalyst sol and the MIMIC techniques.

Among the advantages of these methods are:

1. Non-destructive method for both patterned catalyst and the substrate;

2. Low cost;

3. Can be adopted fast to different type of catalyst precursors;

4. Procedure itself is not time consuming;

5. Provides possibility for wafer scale deposition. 
The minimum achievable dimension is limited by the mask fabrication process and wetting properties of the immobilized catalyst precursors.

By wafer scale deposition we mean (see Figure 7.12):

- Deposition of various catalyst precursors using a single PDMS mold;

- Deposition of one catalyst precursor from the mold replicated from a wafer and placed on a rigid support to ensure mechanical stability.

An example of a machine facilitating wafer scale printing of a catalyst can be found in literature [168].
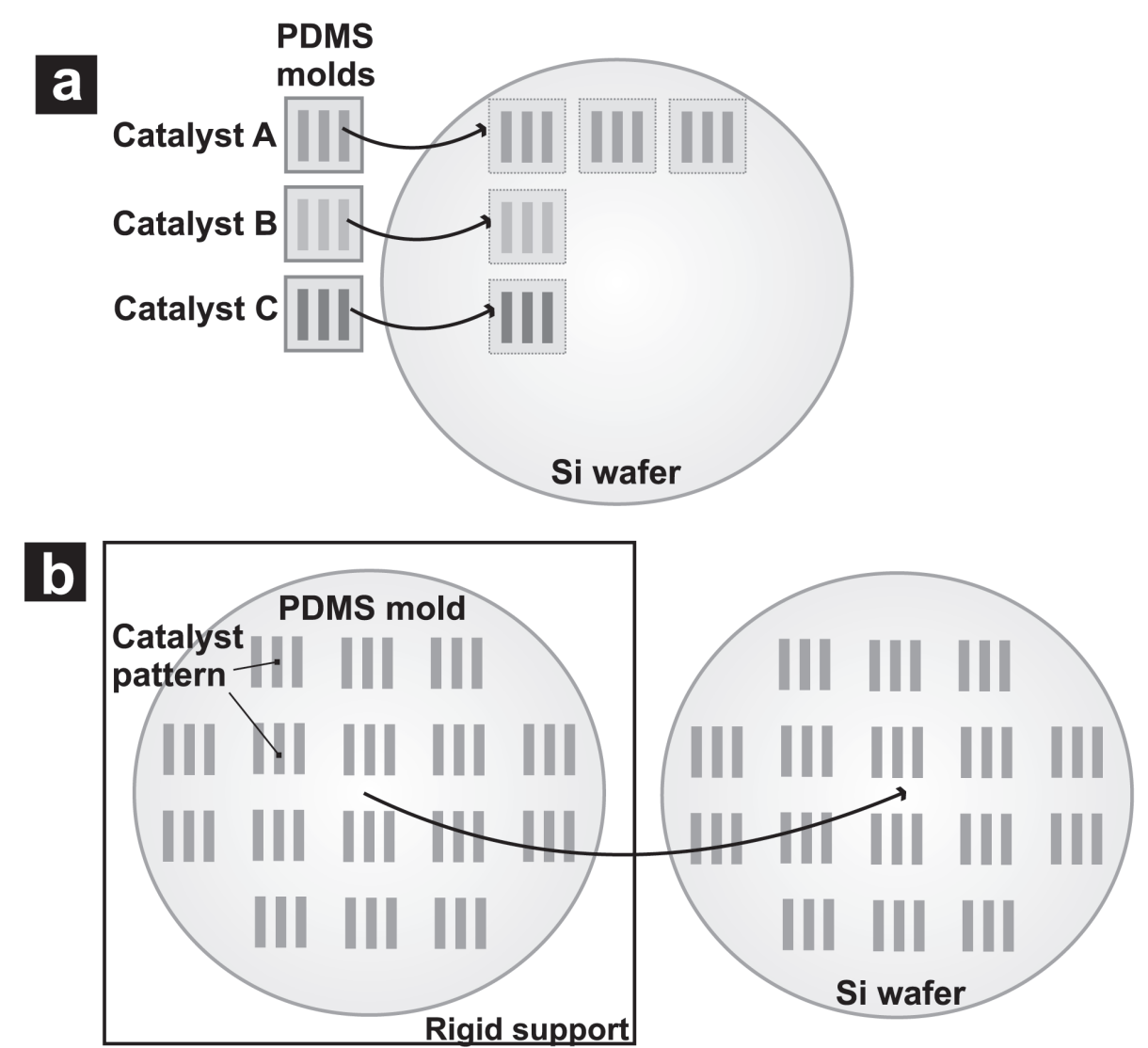

Figure 7.12: Upscaling of soft lithographic techniques to wafer level.

\subsection{Summary and conclusion}

Sputtered thin films and sol-gel based catalyst were investigated as potential functional coatings in micromachined gas sensors. Sputtered thin films are characterized by a low surface area and if, there is a non-active phase present on the top layer, low activity. However, introducing a micropattern may resolve this issue. The catalyst does not require any specific activation procedure and can be used directly. Sputtered thin films can be deposited and patterned in a straightforward way using magnetron sputtering in combination with metal lift-off and photolithography.

Sol-gel based supports ensure a high surface area and a high dispersion, resulting in high activity. Sol-gel based catalyst precursors require the development of specific immobilization techniques in combination with activation procedures (calcination, reduction). Soft-lithographic techniques were applied for the deposition of a boehmite sol.

In terms of reproducibility and efficiency lithography-assisted self-assembly and MIMIC are considered as the 
most promising techniques to pattern a sol-gel based catalyst.

Both the metal thin films as well as the metal dispersed sol-gel based $\gamma$-alumina layers were characterized and showed good catalytic activity (for the results of $\mathrm{CO}$ chemisorption see Table 7.1). Future work will combine these catalyst micropatterns with MEMS-based microcalorimeters to develop catalytic gas sensors [71], [72]. 


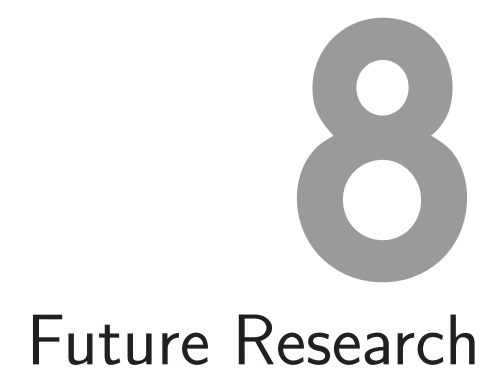

In this chapter we present our perspectives on future research. We address possible issues for improvement of the current design and technology for silicon-based low power hot surface devices. 


\subsection{Design and fabrication of hot surface devices}

In the new hot plate designs these parameters could be interesting to investigate:

- Effect of the heater shape on temperature distribution along the membrane and heater, and, influence on sensitivity;

- Effect of heater and catalyst dimensions on sensitivity;

- Location of heater and temperature sensor relative to each other;

- Integration of several temperature sensors to measure the temperature in the middle of the membrane and at the gas inlet and outlet;

- Different thickness and composition of membrane leading to more robust sensor platform for liquid applications;

- Processing on fused silica and other type of substrates.

- Redesign temperature sensors based on $\mathrm{TiSi}_{2} /$ poly-Si to match the measurement circuit (high-ohmic resistors lead to more accurate measurements).

\subsection{Materials for heating and sensing}

- To complete the knowledge about the $\mathrm{TiSi}_{2} /$ poly-Si temperature sensors the TCR as a function of thicknesses of $\mathrm{TiSi}_{2}$ and poly-Si can be investigated.

\subsection{Experimental}

- Implementation of a differential measurement circuit: differential power (between transducers with and without catalyst patch) in constant temperature mode.

- Introducing splitter and bypass which allows us to set the flows of MFC-s to higher range, i.e. measure more precise (with larger flows error on uncertainty in set point is less), in addition, with these flows it is possible to measure more accurately.

\subsection{Catalytic thin films}

New fabrication methods for the formation of high surface area catalysts in a reproducible way. This can be done, for example, by introducing "defects" and profiles into sputtered thin films. The first ensures presence of highly active catalytic centers, the latter distributes the heat homogeneously and reduces the mechanical stress.

\subsection{Nanolink-based devices}

As far as we can conclude, the link-based device has more potential as a thermal sensor than a thermal actuator. Therefore, in the future study, if application as catalytic gas detector of combustible gases is prioritized, the link heater can be combined with larger flat heater.

For passive temperature sensing applications and those, not exceeding $300{ }^{\circ} \mathrm{C}$, combining links in an array opens possibilities to high resolution temperature measurements. As a lot of nano-scale and low micro-scale effects can not be quantified due to limitation in spacial resolution, ultra-small and ultra-low power heaters can contribute in that. 
Due to their low thermal mass link-based devices are extremely fast, therefore, sensing applications where a rapid response is required, such as flow sensors, are potentially attractive.

The temperature sensitivity of link-based devices is not compatible at this moment with conventional micro hotplates. The fabrication process flow remains challenging and time consuming. The main advantage of this technology is, however, low power consumption. If the sensitivity of the link-based devices can be improved (resistive properties of integrated ALD layers) and process flow simplified, the devices can be potentially useful in various portable sensing systems. 


\section{References}

[1] S. Semancik, R.E. Cavicchi, M.C. Wheeler, J.E. Tiffany, G.E. Poirier, R.M. Walton, J.S. Suehle, B. Panchapakesan, D.L. deVoe, Microhotplate platforms for chemical sensor research, Sens. Actuators B 77 (2001) 579-591.

[2] M. Dijkstra, T.S.J. Lammerink, M.J. de Boer, J.W. Berenschot, R.J. Wiegerink, M.C. Elwenspoek, Low-drift UShaped thermopile flow sensor. In Proceedings of IEEE Sensor Conference, Lecce, Italy (2008) 66-69.

[3] T. Adrega, A.W. van Herwaarden, Chip calorimeter for thermal characterization of bio-chemical solution. Sens. Actuators A 167 (2011) 354-358.

[4] A. Loui, D.J. Sirbuly, S. Elhadj, S.K. McCall, B.R. Hart, T.V. Ratto, Detection and discrimination of pure gases and binary mixtures using a dual-modality microcantilever sensor, Sens. Actuators A 159 (2010) 58-63.

[5] N. Barsan, D. Koziej, U. Weimar, Metal oxide-based gas sensor research: How to ? Sens. Actuators B 121 (2007) 18-35.

[6] A. Hierlemann, Higher-Order Chemical Sensing, Chem. Rev. 108 (2008) 563-613.

[7] D. C. Meier, S. Semancik, B. Button, E. Strelcov, A. Kolmakov, "Coupling nanowire chemiresistors with MEMS microhotplate gas sensing platforms", Appl. Phys. Lett. 91 (2007) 063118.

[8] H.-K. Lee, J.-B. Yoon, E. Yoon, S.-B. Ju, Y.-J. Yong, W. Lee and S.-G. Kim, A high fill-factor infrared bolometer using micromachined multilevel electrothermal structures. IEEE T. Electron Dev. 46(7) (1999) 1489-1491.

[9] M. Zanini-Fisher, High-sensitivity, silicon-based, microcalorimetric gas sensor. US patent 5451371 (1995).

[10] R.E. Cavicchi, G.E. Poirier, N.H. Tea, M. Afridi, D. Berning, A. Hefner, J. Suehle, M.Gaitan, C. Montgomery, Micro-differential scanning calorimeter for combustible gas sensing. Sens. Actuators A 97 (2004) 22-30.

[11] L.F. Houlet, W.S. Shin, K. Tajima, M. Nishibori, N. Izu, T. Itoh, I. Matsubara, Thermopile sensor-devices for the catalytic detection of hydrogen gas. Sens. Actuators B 130 (2008) 200-206.

[12] I. Bársony, M. Ádam, P. Fürjes, R. Lucklum, M. Hirschfelder, S. Kulinyi and C. Dücsô, Efficient catalytic combustion in integrated micropellistors Meas. Sci. Technol. 20 (2009) 1-9.

[13] A. Greve, J. Olsen, N Privorotskaya, L. Senesac, T. Thundat, W.P. King, A. Boisen 2010 Micro-calorimetric sensor for vapor phase explosive detection with optimized heat profile Microelectron. Eng. 87 696-698

[14] C. Dücső, É. V ázsonyi, M. Ádam, I. Szabó, I. Bársony, J.G.E. Gardeniers, A. van der Berg, Porous silicon bulk micromachining for thermally isolated membrane formation. Sens. Actuators A 60 (1997) 235-239.

[15] P.M. Sarro, A.V. van Herwaarden, W. van der Vlist, A silicon-silicon nitride membrane fabrication process for smart thermal sensors. Sens. Actuators A 41(42) (1994) 666-671.

[16] I. Simon, N. Bârsan, M. Bauer, U. Weimar, Micromachined metal oxide gas sensors: opportunities to improve sensor performance. Sens. Actuators B 73(1) (2001) 1-26.

[17] J. Lee, C.M. Spadaccini, E.V. Mukerjee and W.P. King, Differential scanning calorimeter based on suspended membrane single crystal silicon microhotplate. J. Microelectromech. S. 17 (6) (2008) 1513-1525.

[18] J. Lee and N.A. Kotov, "Thermometer design at the nanoscale" Nanotoday 2(1) (2007) 48-51.

[19] A.Y. Kovalgin, J. Holleman, G. Iordache, A pillar-shaped antifuse-based silicon chemical sensor and actuator. IEEE Sens. J. 7 (2007) 18-27.

[20] A.Y. Kovalgin, J. Holleman, G. Iordache, T. Jenneboer, F. Falke, V. Zieren, M.J. Grossens, Low-power antifusebased silicon chemical sensor on a suspended membrane. J. Electrochem. Soc. 153 (2006) H181-H188.

[21] H. Baltes, Thermal CMOS Sensors an Overview. Sensors Update (Wiley) 1 (2001) 121-142.

[22] J. Shieh, J.E. Huber, N.A. Fleck, M.F. Ashby, The selection of sensors. Prog. Mater. Sci. 46 (2001) 461-504 
[23] H. Baltes, CMOS as sensor technology. Sens. Actuators A 37-38 (1993) 51-56.

[24] J.Y.W. Seto, The electrical properties of polycrystalline silicon films. J. Appl. Phys. 46 (1975) 5247-5254.

[25] Mattes et al., Temperature sensor and method. US patent 5.795 .069 (1998).

[26] X. Zhang, A. Mehra, A.A. Ayon, I.A. Waitz, Development of polysilicon igniters and temperature sensors for a micro gas turbine engine. Proceedings of the fifteenth IEEE international conference on micro electro mechanical systems (2002) 280-283.

[27] L. Fonseca, J. Santander, R. Rubio, N. Sabaté, E. Figueras, M. Duch, I. Grácia, C. Cané,Use of boron heavily doped silicon slabs for gas sensors based on free-standing membranes. Sens. Actuators B 130(1) (2008) 538-545.

[28] R.A. Collins, D.F.C. Johnston, G. Dearnaley, Tantalum and cobalt silicides: Temperature sensor applications. Appl. Phys. A-Mater. 40(2) (1986) 109-117.

[29] J.F. Creemer, W. van der Vlist, C.R. de Boer, H.W. Zandbergen, P.M. Sarro, D. Briand, N.F. de Rooij, MEMS hotplates with TiN as a heater material. Proceedings of IEEE Sensors (2005) 330-333.

[30] J.F. Creemera, D. Briand, H.W. Zandbergen, W. van der Vlist, C.R. de Boer, N.F. de Rooij, P.M. Sarro, Microhotplates with TiN heaters. Sens. Actuators A 148(2) (2008) 416-421.

[31] T. Akin, CMOS-based thermal sensors. Advanced Micro and Nanosystems. Vol. 2. CMOS MEMS, Edited by H. Baltes, O. Brand, G. K. Fedder, C. Hierold, J. Korvink, O. Tabata. WILEY-VCH Verlag GmbH \& Co. KGaA, Weinheim 2005.

[32] F. Udrea, S. Santra, J.W. Gardner, CMOS temperature sensors - concepts, state-of-the-art and prospects. Proceedings of International Semiconductor Conference 13-15 Oct. 1 (2008) 31-40.

[33] G.C.M. Meijer, W. Guijie, F. Fruett, Temperature sensors and voltage references implemented in CMOS technology. IEEE Sens. J. 1(3) (2001) 225-234.

[34] A.W. van Herwaarden, Overview of calorimeter chips for various applications. Thermochim. Acta 432(2) (2005) 192-201.

[35] W. Lee, W. Fon, B.W. Axelrod, M.L. Roukes, High-sensitivity microfluidic calorimeters for biological and chemical applications PNAS 106 (2009) 15225-15230.

[36] J.W. Gardner, Microsensors. Wiley (1994).

[37] W. Goepel, J. Hesse, J.N. Zemel, Chemical and biochemical sensors. Part1. Sensors. A comprehensive survey. Vol.2. VCH Verlagsgesellschaft mbH, 1991.

[38] A.R. Baker, Patent GB 892530, Improvements in or relating to electrically heatable filaments. 28, March (1962).

[39] D.W. Dabill, S.L. Gentry, P.T. Walsh, A fast-response catalytic sensor for flammable gases. Sens. Actuators 11 (1987) 135-143.

[40] M.G. Jones, The detection of hydrogen using catalytic flammable gas sensors, Sens. Actuators 16 (1989) 215-224.

[41] C.Vauchier, D.Charlot, G.Delapierre, A.Accorsi, Thin-film gas catalytic microsensor.Sens. Actuators B 5(1-4) (1991) 33-36.

[42] P.Krebs, A. Grisel, A low power integrated catalytic gas sensor. Sens. Actuators B 13-14 (1993) 155-158.

[43] M.Gall, The Si planar pellistor array, a detection unit for combustible gases, Sens. Actuators B 15-16 (1993) 260264.

[44] M. Zanini, J.H. Visser, L. Rimai, R.E. Soltis, A. Kovalchuk, D.W. Hoffman, E.M. Logothetis, U. Bonne, L. Brewer, O.W. Bynum, M.A. Richard, Fabrication and properties of a Si-based high sensitivity microcalorimetric gas sensor. Sens. Actuators A 48 (1995) 187-192. 
[45] S.M. Lee, D.C. Dyer, J.W. Gardner, Design and optinization of a high-temperature silicon micro-hotplate for nanoporous palladium pellistors. Microelectron. J. 34 (2003) 115-126.

[46] P.N. Bartlett, S. Guerin, A micromachined calorimetric gas sensor: an application of electrodeposited nanostructured palladium for the detection of combustible gases. Anal. Chem. 75 (2003) 126-132.

[47] M.-C. Wu, A.L. Micheli, Calorimetric hydrocarbon sensor for automotive exhaust applications. Sens. Actuators B 100 (2004) 291-297.

[48] B.L. Zink, B. Revaz, R. Sappey, F. Hellman, Thin film microcalorimeter for heat capacity measurements in high magnetic fields. Rev. Sci. Instrum. 73(4) (2002) 1841-1844.

[49] W. Shin, M. Matsumiya, N. Izu, N. Murayama, Hydrogen-selective thermoelectric gas sensor. Sens. Actuators $B$ 93 (2003) 304-308.

[50] E.-B. Lee et al., Micromachined catalytic combustible hydrogen gas sensor. Sens. Actuators B 153 (2011) 392-397.

[51] E.A. Johanssen, J.M.R. Weaver, L. Bourova, P. Svoboda, P.H. Cobbold, J.M. Cooper, Micromachined nanocalorimetric sensor for ultra-low-volume cell-based assays. Anal. Chem. 74 (2002) 2190-2197.

[52] E. Iervolino, A.W. van Herwaarden, P.M. Sarro, Calorimeter chip calibration for thermal characterization of liquid samples. Thermochim. Acta 492(2009)95-100.

[53] K. Verhaegen, K. Bert, J. Simaels, W. van Drissche, A high-throughput silicon microphysiometer, Sens. Actuators 82 (2000) 186-190.

[54] F. Yi, D.A. La Van, Nanoscale thermal analysis for nanomedicine by nanocalorimetry. Wiley Interdisciplinary Reviews: Nanomedicine and Nanobiotechnology (2011) John Wiley \& Sons, Inc pp. 1939-1941.

[55] M. Graf, U. Frey, S. Taschini, A. Hierlemann, Micro hot plate-based sensor array system for the detection of environmentally relevant gases, Anal. Chem. 78(19) (2006) 6801-6808.

[56] B. Morana, F. Santagata, L. Mele, M. Mihailović, G. Pandraud, J.F. Creemer, P.M. Sarro, A silicon carbide MEMS microhotplate for nanomaterial characterization in TEM, Proceed. MEMS Conference, 23-27 Jan. (2011) 380-383.

[57] N.L. Privorotskaya, W.P. King, Silicon microcantilever hotplates with high temperature uniformity, Sens. Actuators A 152 (2009) 160-167.

[58] D. M. Wilson, S. Hoyt, J. Janata, K. Booksh, L. Obando, Chemical sensors for portable, handheld field instruments, IEEE Sens. J. 1(4) (2001) 256-274.

[59] R.E. Cavicchi, G.E. Poirier, J.S. Suehle, M. Gaitan, S. Semancik, D.R.F. Burgess, Pulsed desorption kinetics using micromachined microhotplate arrays, J. Vac. Sci. Technol. A 12(4) (1994) 2549-2553.

[60] A.G. Shirke, R.E. Cavicchi, S. Semancik, R.H. Jackson, B.G. Frederick, M.C. Wheeler, Femtomolar isothermal desorption using microhotplate sensors, J. Vac. Sci. Technol. A 25 (2007) 514-526.

[61] K.D. Hurley, B.G. Frederick, W.J. De Sisto, A.R.P. van Heiningen, M.C. Wheeler, Catalytic reaction characterization using micromachined nanocalorimeters, Appl. Catal. A 290(1-2) (2010) 84-93.

[62] J.K. Olsen, A. Greve, A. Boisen, N. Privorotskaya, W.P. King, L. Senesac, T. Thundat, Micro calorimetric sensor for trace explosive particle detection, Micro- and Nanotechnology Sensors, Systems, and Applications ||, Proc. of SPIE (7679) (2010) 767929.

[63] S.-I. Yoon et al., Neisseria meningitidis detection based on a microcalorimetric biosensor with a split-flow microchannel, J. Microelectromech. S. 17 (3) (2008) 590-598.

[64] B.S. Kwak et al., Dual thermopile integrated microfluidic calorimeter for biochemical thermodynamics, Microfluid. Nanofluid. 5 (2008) 255262.

[65] R.M. Tiggelaar et al., Fabrication of a high-temperature microreactor with integrated heater and sensor patterns on an ultrathin silicon membrane, Sens. Actuators A 19(1) (2005) 196-205. 
[66] R.M. Tiggelaar, P.W.H. Loeters, P. van Male, R.E. Oosterbroek, J.G.E. Gardeniers, M.H.J.M. de Croon, J.C. Schouten, M.C. Elwenspoek, A. van den Berg, Thermal and mechanical analysis of a microreactor for high temperature catalytic gas phase reactions, Sens. Actuators A 112 (2004) 267277.

[67] S.M. Lee, D.C. Dyer, J.W. Gardner, Design and optimization of a high-temperature silicon micro-hotplate for nanoporous palladium pellistors,Microelectron. J. 34 (2003) 115-126.

[68] M. Graf, D. Barettino, H.P. Baltes, A. Hierlemann, CMOS hotplate chemical microsensors, Springer Verlag Berlin Heidelberg (2007) 17-28.

[69] P. van Male, M.H.J.M. de Croon, R.M. Tiggelaar, A. van der Berg, J.C. Schouten, Heat and mass transfer in a square microchannel with assymetric heating, Int. J. Heat Mass Tran., 47(1) (2004) 87-99.

[70] S. Karagiannidis, K. Marketos, J. Mantzaras, R. Schaeren, K. Boulouchos, Experiemental and numerical investigation of a propane-fueled, catalytic mesoscale combustor, Catal. Today, 155 (2010) 108-115.

[71] E. Vereshchagina, R.A.M. Wolters, J.G.E. Gardeniers, Measurement of reaction heats using a polysilicon-based microcalorimetric sensor, Sens. Actuators A 169(2) (2011) 308-316.

[72] E. Vereshchagina, R.A.M. Wolters, J.G.E. Gardeniers, The development of titanium silicide boron-doped polysilicon resistive temperature sensors, J. Micromech. Microeng. 21 (2011) 105022.

[73] A.D. McConnell, S. Uma, Thermal conductivity of doped polysilicon layers, J. Microelectromech. S., 10(3) (2001) $360-369$.

[74] B. Bhushan, Handbook of nanotechnology, $2^{\text {nd }}$ edition Springer (Berlin) (2007).

[75] Handbook of chemistry and physics, $9_{\text {th }}$ edition, http://www.hbcpnetbase.com/ (2010-2011).

[76] F.P. Incropera et al, Fundamentals of Heat and Mass Transfer, John Wiley \& Sons (2011).

[77] I.Barin, Thermochemical data of pure substances, VCH Verlagsgesellschaft (Weinheim) (1995).

[78] http://www.mit.edu/ 6.777/matprops/pecvd_sin.htm

[79] http://www.mit.edu/ 6.777/matprops/pecvd_sio2.htm

[80] C.H. Mastrangelo, Y.-C. Tai, R.S.Muller, Thermophysical properties of low-residual stress, silicon-rich, LPCVD silicon nitride films, Sens. Actuators A21-A23 (1990) 856-860.

[81] R. Sultan, A.D. Avery, G. Stiehl, B. Zink, Thermal conductivity of micromachined low-stress silicon-nitride beams from 77 to 325 K. J. Appl. Phys. 105 (2009) 043501.

[82] S.-M. Lee, D.G. Cahill, Heat transport in thin dielectric films. J. Appl. Phys. 81 (6) (1997) 2590-2595.

[83] C. Prakash, Thermal conductivity variation of silicon with temperature, Microelectron. Reliab., 18(4) (1978) 333.

[84] P. Furjes, P. Csikvari, I. Barsony, C. Ducso, Micro-hotplates for thermal characterisation of structural materials of MEMS. (2007) THERMINIC 13th International Workshop on Thermal Investigation of ICs and Systems, , 17-19 Sept. 2007, 184-188

[85] D. Briand, S. Heimgartner, M.A. Gretillat, B. van der Schoot, N.F. de Rooj, Thermal optimization of microhotplates that have a silicon island, J. Micromech. Microeng. 12 (2002) 917-978.

[86] L. van de Beld, M.C. van der Ven, K.R. Westerterp, A kinetic study of the complete oxidation of ethene, propane and their mixtures on a $\mathrm{Pd} / \mathrm{Al}_{2} \mathrm{O}_{3}$ catalyst, Chem. Eng. Process. 34 (1995) 469-478.

[87] I. Chorkendorff, J.W. Niemantsverdriet, Concepts of modern catalysis and kinetics. Wiley-VCH (2003).

[88] A. Hierlemann, D. Lange, C. Hagleitner, N. Kerness, A. Koll, O. Brand, H. Baltes, Application-specific sensor systems based on CMOS chemical microsensors, Sens. Actuators, 70 (2000) 2-11. 
[89] M. Dijkstra, T.S.J. Lammerink, M.J. de Boer, M.J. Berenschot, R.J. Wiegerink, M. Elwenspoek, Low-drift flow sensor with zero-offset thermopile-based power feedback, In Proc. DTIP of MEMS and MOEMS (2008).

[90] P. Bataillard, Calorimetric sensing in bioanalytical chemistry: principles, applications and trends, Trends in analytical chemistry 12(10) (1993) 387-394.

[91] D.J. Quiram, K.F. Jensen, M.A. Schmidt, P.L. Mills, J.F. Ryley, M.D. Wetzel, D.J. Kraus, Integrated microreactor system for gas-phase catalytic reactions. 1. Scale-up microreactor design and fabrication, Ind. Eng. Chem. Res. 46 (2007) 8292-8305.

[92] D.J. Quiram, K.F. Jensen, M.A. Schmidt, P.L. Mills, J.F. Ryley, M.D. Wetzel, D.J. Kraus, Integrated microreactor system for gas-phase catalytic reactions. 2. Microreactor packaging and testing, Ind. Eng. Chem. Res. 46 (2007) 8306-8318.

[93] D.J. Quiram, K.F. Jensen, M.A. Schmidt, P.L. Mills, J.F. Ryley, M.D. Wetzel, D.J. Kraus, Integrated microreactor system for gas-phase catalytic reactions. 3. Microreactor system design and system automation, Ind. Eng. Chem. Res. 46 (2007) 8319-8335.

[94] C.F. Cullis, T.G. Nevell, The Kinetics of the catalytic oxidation over palladium of some alkanes and cycloalkanes, P. Roy. Soc. Lond. A Mat. 349(1659) (1976) 523-534.

[95] L. Hiam, H. Wise, S. Chaikin, Catalytic oxidation of hydrocarbons on platinum, J. Catal. 9(10) (1968) $272-276$.

[96] E. Obermeier, P. Kopystynski, Polysilicon as a material for microsensor applications, Sens. Actuators, A 30 (1992) 149-155.

[97] J. Xie, C. Lee, M.-F. Wang, Y. Liu, H. Feng, Characterization of heavily doped polysilicon films for CMOS-MEMS thermoelectric power generators, J. Micromech. Microeng. 19 (2009) 125029.

[98] I. Erill, S. Campoy, J. Rus, L. Fonseca, A. Ivorra, Z.Navarro, J.A. Plaza, J. Aguilo, J.Barbe, Development of a CMOS-compatible PCR chip: comparison of design and system strategies, J. Micromech. Microeng. 14 (2004) 15581568.

[99] S. Jae Oh, J. Keun Song, S. Kwan An, S. June Kim, Properties of the doped polycrystalline silicon as site material for the micromachined silicon neural probe, J. Mater. Sci. Lett. 22 (2003) 131133.

[100] C.-I. Baban, Y. Toyoda, M. Ogita, High temperature oxygen sensor using a $\mathrm{PtGa}_{2} \mathrm{O}_{3} \mathrm{Pt}$ sandwich structure, Jpn. J. Appl. Phys. 43 (2004) 7213-7216.

[101] J. Courbat, D. Briand, N.F. de Rooij, Reliability improvement of suspended platinum-based micro-heating elements, Sens. Actuators, A 142 (2008) 284291.

[102] M. Ehmann, P. Ruther, M. von Arx, O. Paul, Operation and short-term drift of polysilicon-heated CMOS microstructures at temperatures up to 1200 K, J. Micromech. Microeng. 11 (2001) 397401.

[103] J. Lee, C.M. Spadaccini, E.V. Mukerjee and W.P. King, Differential scanning calorimeter based on suspended membrane single crystal silicon microhotplate, J. Microelectromech. S. 17 (6) (2008) 1513-1525.

[104] R.M. Tiggelaar, R.G.P. Sanders, A.W. Groenland, J.G.E. Gardeniers, Stability of thin platinum films implemented in high-temperature microdevices, Sens. Actuators, A 152 (2009) 39-47.

[105] R.M. Tiggelaar, A.W. Groenland, R.G.P. Sanders and J.G.E. Gardeniers, Electrical properties of low pressure chemical vapor deposited silicon nitride thin films for temperatures up to 650 $\mathrm{C}$, J. Appl. Phys. 105033714 (2009).

[106] S. Enderling, C.L. Brown, S. Smith, M.H. Dicks, J.T.M. Stevenson, M. Mitkova, M.N. Kozicki, A.J. Walton, Sheet resistance measurement of non-standard cleanroom materials using suspended greek cross test structures, IEEE T. Semiconduct. M. 19 (2006) 2-9.

[107] M.M. Mandurah, K.C. Saraswat, C.R. Heims, Dopant segregation in polycrystalline silicon, J. Appl. Phys. 51(11) (1980) 5755-5763. 
[108] T.I. Kamins, Polycrystalline silicon for integrated circuits and displays, 2nd edition, Kluwer Academic Publishers (1998).

[109] Z. Xue, H. Qiu, Integrating micromachined fast response temperature sensor array in a glass microchannel, Sens. Actuators, A 122(2) (2005) 189-195.

[110] A. Andrei, Long-term stability of metal Lines, polysilicon gauges, and ohmic contacts for harsh-environment pressure sensors. IEEE Sens. J. 6(6) (2006) 1596-1601.

[111] N. Sabate, J. Cerda, I. Gracia, J. Berganzo, C. Cane, J.R. Morante, Evaluation of sensitive materials for integrated thermal flow sensors, In Proc. IECON IEEE 28th Annual conference of the industrial electronics society 4 (2002) 2681-2684.

[112] R.M. Tiggelaar, J.W. Berenschot, J.H. de Boer, R.G.P. Sanders, J.G.E. Gardeniers, R.E. Oosterbroek, A. van den Berg and M.C. Elwenspoek, Fabrication and characterization of high-temperature microreactors with thin film heater and sensor patterns in silicon nitride tubes, Lab. Chip 5 (2005) 326-336.

[113] K. Arnby, J. Assiks, P.-A. Carlsson, A. Palmqvist, M. Skoglundh, The effect of platinum distribution in monolithic catalysts on the oxidation of CO and hydrocarbons, J. Cat. 233 (2005) 176-185.

[114] J. Riegel, K.H. Härdtl, Analysis of combustible gases in air with calorimetric sensors based on semiconducting $\mathrm{BaTiO}_{3}$ ceramics, Sens.Actuators, B (1990) 54-51.

[115] R.M. Tiggelaar, P.W.H. Loeters, P. van Male, R.E. Oosterbroek, J.G.E. Gardeniers, M.H.J.M. de Croon, J.C. Schouten, M.C. Elwenspoek and A. van den Berg, Thermal and mechanical analysis of a microreactor for high temperature catalytic gas phase reactions, Sens. Actuators A 112 (2004) 267-277

[116] S. Jensen, S. Thorsteinsson, O. Hansen and U.J. Quaade, Parametric investigation of rate enhancement during fast temperature cycling of CO oxidation in mircroreactors, Chem. Eng. J. 135S (2008) S237-S241

[117] N. Wilken, K. Kamasamudram, N.W. Currier, J. Li, A. Yezerets and L. Olsson, Heat of adsorption for $\mathrm{NH}_{3}, \mathrm{NO}_{2}$ and $\mathrm{NO}$ on $\mathrm{Cu}$-Beta zeolite using microcalorimeter for $\mathrm{NH}_{3}$ SCR applications, Catal. Today 151 (2010) 237-243

[118] H. Baltes, O. Paul and D. Jaeggi, Thermal CMOS Sensors an Overview, Sensors Update (Wiley) 1 (2001) 121-142

[119] C.H. Ho, Y.H.C. Cha, S. Prakash, G. Potwin, H.J. Doerr, C.V. Deshpandey, R.F. Bunshah, M. Zeller, Electrical resistance drift of molybdenum silicide thin film temperature sensors, Thin Solid Films 260 (1995) 232-238

[120] S. Jensen, High-temperature compatible nickel silicide thermometer and heater for catalytic chemical microreactors, Proc. of 18th International Conference on Micro Electro Mechanical Systems 24-27 July, 2005, Banff, Alberta, Canada 463-466

[121] L.W.-W. Fang, R. Zhao, E.-G. Yeo, K.-G. Lim, H. Yang, L. Shi, T.-C. Chong and Y.-C. Yeo, Phase change random access memory devices with nickel silicide and platinum silicide electrode contacts for integration with CMOS technology J. Electrochem. Soc. 158 (2011) H232-H238

[122] L.J. Chen, Silicide technology for integrated circuits, (London: The institution of electrical engineers/UK), (2004)

[123] K. Wetzig, Metal based thin films for electronics, Weinheim: Germany/WILEY-VCH Verlag Gmbh \& Co. KGaA, (2006) 48-64

[124] S.P. Murarka, Silicide thin films and their applications in microelectronics Intermetallics 3 (1995) 173-186

[125] A.H. Reader, Transition metal silicides in silicon technology Rep. Prog. Phys. 56 (1992) 1397-1467

[126] S.L. Zhang and M. Ostling, Metal Silicides in CMOS Technology: Past, Present, and Future Trends, Crit. Rev. Solid State 28 (2003) 1-129

[127] J.P. Gambino, E.G. Colgan, Silicides and ohmic contacts, Mater. Chem. Phys. 52 (1998) 99-146

[128] Z. Ma and L.H. Allen, Kinetic mechanisms of the C49-to-C54 polymorphic transformation in titanium disilicide thin films: a microstructure-scaled nucleation-mode transition, Phys. Rev. B 49 (1994) 13501-13511 
[129] F. Mammoliti, M.G. Grimaldi and F. La Via, Electrical resistivity and Hall coefficient of C49, C40, and C54 TiSi 2 thin-film phases, J. Appl. Phys. 92 (2002) 3147-3151

[130] M. Bhaskaran, S. Sriram and L.W. Sim, Nickel silicide thin films as masking and structural layers for silicon bulk micro-machining by potassium hydroxide wet etching, J. Micromech. Microeng. 18 (2008) 095002-095004

[131] Z. Li, G. Zhang, W. Wang, Y. Hao, T. Li and G. Wu, Study on the application of silicide in surface micromachining, J. Micromech. Microeng. 12 (2002) 162-167

[132] M. Qin, M.C. Poon and C.Y. Yuen, A study of nickel silicide film as a mechanical material, Sens. Actuators, 87 (2000) 90-95

[133] P. Ritterskamp, A. Kuklya, M.-A. Wüstkamp, K. Kerpen, C. Weidenthaler and M. Demuth, A titanium disilicide derived semiconducting catalyst for water splitting under solar radiation-reversible storage of oxygen and hydrogen, Angew. Chem. Int. Ed. 46 (2007) 7770-7774

[134] E.G. Colgan, G.P. Gambino, Q.Z. Hong, Formation and stability of silicides on polycrystalline silicon, Mater. Sci. Eng. R16 (1996) 43-96

[135] Y.M. Chen, G.C. Tu, Y.L. Wang, G.L. Hwang and C.Y. Lo, CoSi $x_{x}$ thermal stability on narrow-width polysilicon resistors, J. Vac. Sci. Technol. B 24 (2006) 83-86

[136] T.E. Karlin, S. Nygren, L. Mattsson, E. Andre, M. Bjuggren and F.M. dHeurle, The influence of silicon substrate crystallinity and doping on TiSi 2 thin film morphology, Appl. Surf. Sci. 73 (1993) 280-284

[137] W. Wong-Ng, H. McMurdie, B. Peretzkin, C. Hubbard and A. Dragoo, Powder Diffr. 2

[138] N.V. Ageev and V.P. Samsonov, Zh. Neorg. Khim. 41590

[139] J.A. Kittl, M.A. Gribelyuk and S.B. Samavedam, Mechanism of low temperature C54 $\mathrm{TiSi}_{2}$ formation bypassing C49 TiSi 2 : Effect of Si microstructure and Mo impurities on the TiSi reaction path, Appl. Phys. Lett. 73 (1998) 900-902

[140] D. Barrettino, M. Graf, M. Zimmermann, C. Hagleitner, A. Hierlemann, H. Baltes, A smart single-chip microhotplate-based gas sensor system in CMOS-technology. Analog Integr. Circ. S. 39 (2004) 275-287.

[141] Y. Mo, Y. Okawa, K. Inoue, K. Natukawa, Low-voltage and low-power optimization of micro-heater and its on-chip drive circuitry for gas sensor array. Sens. Actuators A 100(1) (2002) 94-101.

[142] B. Guo, A. Bermak, P. C. H. Chan, G.-Z. Yan, An integrated surface micromachined convex microhotplate structure for tin oxide gas sensor array. IEEE Sens. J. 7(12) (2007) 1720-1726.

[143] S. Semancik, R.E. Cavicci, M.C. Wheeler, J.E. Tiffany, G.E. Poirier, R.M. Walton, J.S. Suehle, B. Panchapakesan, D.L. DeVoe, Microhotplate platforms for chemical sensor research. Sens. Actuators B 77 (2001) 579-591.

[144] M.Y. Afridi, J.S. Suehle, M.E. Zaghloul, D.W. Berning, A.R. Hefner, R.E. Cavicchi, S.Semancik, C.B. Montgomery, C.J. Taylor, A monolithic CMOS microhotplate-based gas sensor system. IEEE Sens. J. 2(6) (2002) 644655 .

[145] E. Zhuravlev, C. Schick, Fast scanning power compensated differential scanning nano-calorimeter: 1. The device. Thermochim. Acta 505(1-2) (2010) 1-13.

[146] R.K. Kummamuru, L. De La Rama, L. Hu, M.D. Vaudin, M.Y. Efremov, M.L. Green, D.A. LaVan, L.H. Allen, Measurement of heat capacity and enthalpy of formation of nickel silicide using nanocalorimetry. Appl. Phys. Lett. 95 (2009) 181911.

[147] A.Y. Kovalgin, J. Holleman, G. Iordache, T. Jenneboer, F. Falke, V. Zieren and M.J. Goossens, ”Low-Power, Antifuse-Based Silicon Chemical Sensor on a Suspended Membrane" J. Electrochem. Soc. 153(9) (2006) H181H188.

[148] A.W. Groenland, R.A.M. Wolters, A.Y. Kovalgin and J. Schmitz, "Nano-link based ultra low power micro electronic hotplates for sensors and actuators", ECS transactions (2011) 35. 
[149] A.W. Groenland, "Nanolink-based thermal devices: integration of ALD TiN thin films" PhD thesis, University of Twente, The Netherlands (2011).

[150] H.-Y. Lee, S. Moon, S.J. Park, J. Lee, K.-H. Park and J. Kim, ”Micro-machined resistive micro-heaters for high temperature gas sensing applications", Electron. Lett 44(25) (2008).

[151] J.G. Firth, A. Jones, T.A. Jones, Combustion and flame 20(3) (1973) 303-311.

[152] J.J. Bravo-Surez, K.K. Bando, J. Lu, T. Fujitani, S.T. Oyama, Oxidation of propane to propylene oxide on gold catalysts. J. Catal. 255(1) (2008) 114-126.

[153] M. Taylor, E. N. Ndifor, T. Garcia, B. Solsona, A.F. Carley, S.H. Taylor, Deep oxidation of propane using palladiumtitania catalysts modified by niobium. Appl. Catal. A 350(1) (2008) 63-70.

[154] H. Shinjoh, H. Muraki, Y. Fujitani, Periodic operation effects in propane and propylene oxidation over noble metal catalysts. Appl. Catal. 49(2) (1989) 195-204.

[155] L. Hiam, H. Wise, S. Chaikin, Catalytic oxidation of hydrocarbons on platinum. J. Catal. 9(10) (1968) $272-276$.

[156] J. Hermia, S. Vigneron, Catalytic incineration for odour abatement and VOC destruction. Catal. Today 17 (1993) $349-358$.

[157] J.J. Spivey, Complete catalytic oxidation of volatile organics. Ind. Eng. Chem. Res. 26 (1987) 2165-2180.

[158] T.A. Jones, P.T. Walsch, Flammable gas detection. The role of platinum metals. Platinum Metals Review 32(2) (1988) 50-60.

[159] H.S. Gandhi, G.W. Graham, R.W. McCabe, Automotive exhaust catalysis. J. Catal. 216(1-2) (2003) $433-442$.

[160] J.E. ten Elshof, S.U. Khan, O.F. Göbel, Micrometer and nanometer-scale parallel patterning of ceramic and organicinorganic hybrid materials. J. Eur. Ceram. Soc. 30(7) (2010) 1555-1577.

[161] K. Arnby, J. Assiks, P.-A. Carlsson, A. Palmqvist, The effect of platinum distribution in monolithic catalysts on the oxidation of CO and hydrocarbons. J. Catal. 233 (2005) 176-185.

[162] H.L. Tuller, R. MlCac, Advanced sensor technology based on oxide thin film - MEMS integration J. Electroceram. $4: 2 / 3$ (2000) 415-425.

[163] M. Roumanie, C. Pijolat, V. Meille, C. De Bellefon, P. Pouteau, C. Delattre, Deposition of Pt-catalyst in a microchannel of a silicon reactor: Application to gas micro-TAS working at high temperature. Sens. Actuators B 118 (2006) 297-304.

[164] V. Meille, Review on methods to deposit catalysts on structured surfaces. Appl. Catal. A: General 315 (2006) 1-17.

[165] P. Fürjes, M. Ádám, Cs. Dücső , J. Zettner, I. Bársony, Thermal effects by the sensitive coating of calorimetric gas sensors. Sens. Actuators B 111112 (2005) 96-101.

[166] S.R. Quake, A. Scherer, From micro- to nanofabrication with soft materials. Science 290 (2000) 1536-1540.

[167] Y. Xia, G. M. Whitesides, Soft Lithography, Angew. Chem. Int. Ed. 37 (1998) 550-575.

[168] J.A. Rogers, R.G. Nuzzo, Recent progress in soft lithography. Mater. Today 8(2) (2005) 50-56.

[169] B. Michel et al., Printing meets lithography: soft approaches to high-resolution patterning. IBM J. Res. \& Dev. 45(5) (2001) 697-719.

[170] H. Fudouzi, Y. Sakka, Micro gas sensor assembly of tin-oxide nanoparticles by a capillary micro-moulding process. J. Ceram. Soc. Jpn. 118(3) (2010) 202-205.

[171] A. Perl, D.N. Reinhoudt, J. Huskens, Microcontact Printing: Limitations and Achievements. Adv. Mater. 21 (2009) 2257-2268. 
[172] E. Kim, Y.N. Xia, G.M. Whitesides, Polymer microstructures formed by molding in capillaries. Nature 376 (1995) 581-584.

[173] M. Heule, L.J. Gauckler, Gas sensors fabricated from ceramic suspensions by micromolding in capillaries. Adv. Mater. 13(23) (2001) 1790-1793.

[174] M. Heule, L.J. Gauckler, Miniaturised arrays of tin oxide gas sensors on single microhotplate substrates fabricated by micromolding in capillaries. Sens. Actuators B 93 (2003) 100-106.

[175] Y.S. Lin, A.G. Burggraaf, Preparation and characterization of high temperature thermally stable alumina composite membrane. J. Am. Ceram. Soc. 74(1) (1991) 219-224.

[176] P.M. Biesheuvel, H. Verweij, Design of ceramic membrane supports: permeability, tensile strength and stress. $J$. Membrane Sci. 156 (1999) 141-152.

[177] P.G. de Gilles, F. Brochard-Wyart, D. Cuéré, Capillarity and wetting phenomena. (2004) Springer Science.

[178] T.M. Mayer, M.P. de Boer, N.D. Shinn, P.J. Clews, T.A. Michalske, Chemical vapor deposition of fluoroalkylsilane monolayer films for adhesion control in microelectromechanical systems, J. Vac. Sci. Technol. B 18(5) (2000) 2433-2440.

[179] H. Rathgen, Superhydrophobic surfaces : from fluid mechanics to optics (2008), PhD Thesis, University of Twente, The Netherlands.

[180] N. Tian, Z.-Y. Zhou, S.-G. Sun, Platinum metal catalysts of high-index surfaces: from single-crystal planes to electrochemically shape-controlled nanoparticles. J. Phys. Chem. C 112 (2008) 19801-19817.

[181] P. Gélin, M. Primet, Complete oxidation of methane at low temperature over noble metal based catalysts: a review. Appl. Catal. B: Environmental 39 (2002) 1-37.

[182] O. Bliznyuk, E. Vereshchagina, E.S. Kooij, P. Poelsema, Scaling of anisotropic droplet shapes on chemically stripepatterned surfaces. Phys. Rev. E 79 (6) (2009) 041601 1-5.

[183] O. Bliznyuk, H.P. Jansen, E.S. Kooij, H.J.W. Zandvliet, B. Poelsema, Initial spreading kinetics of high-viscosity droplets on anisotropic surfaces. Langmuir 26(6) (2009) 6328-6334.

[184] M. Heule, U. P. Schönholzer, L.J. Gauckler, Patterning colloidal suspensions by selective wetting of microcontactprinted surfaces. J. Eur. Ceram. Soc. 24(9) (2004) 2733-2739. 


\section{Appendix}

Appendix A

(a) Temperature dependence of thermal conductivity of $\mathrm{Si}, \mathrm{SiO}_{2}$ and $\mathrm{Si}_{3} \mathrm{~N}_{4}$.

The following polynom fits were incorporated in the model:

$\mathrm{f}_{f i t}\left(\mathrm{~T}_{S i}\right)=-6 \cdot 10^{-7} \cdot \mathrm{T}^{3}+1.5 \cdot 10^{-3} \cdot \mathrm{T}^{2}-1.27 \cdot \mathrm{T}+418.32$,

$\mathrm{f}_{f i t}\left(\mathrm{~T}_{\mathrm{SiO} 2}\right)=3 \cdot 10^{-9} \cdot \mathrm{T}^{3}-3 \cdot 10^{-6} \cdot \mathrm{T}^{2}+2.4 \cdot 10^{-3} \cdot \mathrm{T}+0.88$,

$\mathrm{f}_{f i t}\left(\mathrm{~T}_{\text {Si3N }}\right)=-4 \cdot 10^{-9} \cdot \mathrm{T}^{3}+2 \cdot 10^{-5} \cdot \mathrm{T}^{2}-26.9 \cdot 10^{-3} \cdot \mathrm{T}+22.43$.

(b) Temperature dependence of specific heat capacity of $\mathrm{Si}, \mathrm{SiO}_{2}$ and $\mathrm{Si}_{3} \mathrm{~N}_{4}$.

The following polynomial fits were incorporated in the model:

$\mathrm{f}_{f i t}\left(\mathrm{~T}_{S i}\right)=-6 \cdot 10^{-7} \cdot \mathrm{T}^{3}+1.5 \cdot 10^{-3} \cdot \mathrm{T}^{2}-1.27 \cdot \mathrm{T}+418.32$,

$\mathrm{f}_{f i t}\left(\mathrm{~T}_{S i O 2}\right)=3 \cdot 10^{-9} \cdot \mathrm{T}^{3}-3 \cdot 10^{-6} \cdot \mathrm{T}^{2}+2.4 \cdot 10^{-3} \cdot \mathrm{T}+0.88$,

$\mathrm{f}_{f i t}\left(\mathrm{~T}_{S i 3 N 4}\right)=-4 \cdot 10^{-9} \cdot \mathrm{T}^{3}+2 \cdot 10^{-5} \cdot \mathrm{T}^{2}-26.9 \cdot 10^{-3} \cdot \mathrm{T}+22.43$.
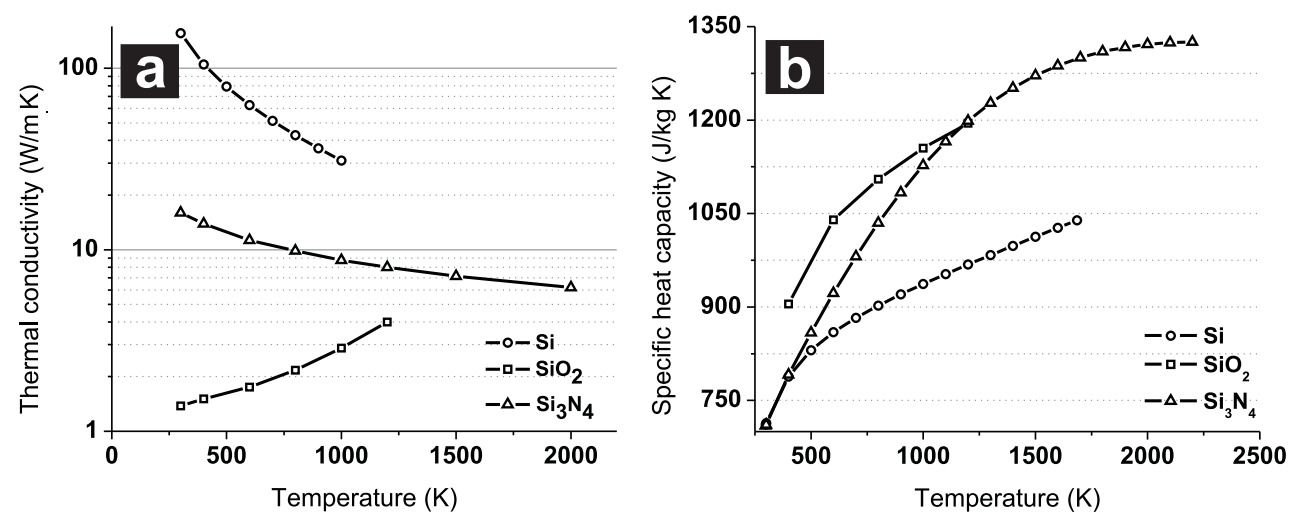



\section{Summary}

In the Hot Silicon project low and ultra-low-power Si-based hot surface devices have been developed, i.e. thermal sensors and actuators, for application in catalytic gas micro sensors, micro- and nano- calorimeters.

This work include several scientific and technological aspects:

- Design and fabrication of suspended membrane ultra-low-power hot-surface Si-based thermal sensors and actuators;

- Integration of the sensors and actuators into a micro flow channel, and investigation of the related microfluidic aspects, i.e. flow behavior and control, heat transport and transport of reactive species,

- Investigation of potential applications in the field of micro calorimetric sensing, e.g. studying thermally-activated physical and chemical processes on-a-chip, i.e. detection of reaction heats, adsorption/desorption kinetics.

A numerical model for a catalytic micro flow system consisting of a micro hotplate at elevated temperature with an immobilized reactive catalyst has been developed. The influence of the micro hotplate design parameters, such as, thickness and material of an isolating membrane, dimensions of the etched cavity in silicon substrate, type of substrate on power consumption, overall thermal efficiency, heat loss mechanism and surface temperature distribution has been investigated. The material of the membrane influences heat loss mechanisms more than the thickness of the membrane with all other constant parameters. The implementation of thinner membranes and membranes of low thermally conductive materials both leads to a decrease in the total power consumption but also leads to non-uniformity in surface temperature distribution. The ratio of heater area to membrane, $\mathrm{a}_{h} / \mathrm{a}_{m}$, taken below 0.25 reduces the heat flux to the substrate and does not lead to sharp surface temperature profiles. The main heat loss paths are by Si substrate (ca. $40 \%$ ) and air (ca. $60 \%$ ), the amount of heat lost through the membrane is less than $1 \%$ of total supplied power. However, the membrane introduces a heat path to the $\mathrm{Si}$, thus, the thickness, and especially the material of the membrane need to be selected based on the application specific power consumption requirements.

The heating effect of a reaction is directly proportional to the concentration of a target gas (propane) in air, and actuating temperature. If a catalyst is not active enough, higher temperatures can be applied to compensate for it. Non-flow operating conditions are the preferred operational mode because losses are less compared to constant flow operation. From the actuation point of view it does not make sense to downscale the dimensions of the heater to nanometer range while keeping constant the size of the catalytic patch. A small heater would be not capable of delivering sufficient power to heat up the catalyst patch. If the catalyst is much smaller than the heater than it also not favorable situation as heat will be just generated and lost to the air. A preferred design is where heater and catalyst are of the same dimensions or $\mathrm{a}_{c a t} / \mathrm{a}_{h}$ below 2.5. A small resistive element when integrated with a powerful heater can be used as a temperature sensor.

The fabrication, characterization and testing of a low-cost, low-power, CMOS-compatible microcalorimetric sensor for detection of reaction heats is discussed.

The construction of the calorimetric sensing system based on extrinsically-doped polysilicon resistive heaters and temperature sensors has been demonstrated. The compatibility with Si technology and the chemical inertness at elevated temperatures makes polysilicon attractive for integration into microreactors and sensors. However, non-linearity of its resistive properties and drift still offer challenges to its successful implementation as a functional sensing material.

We demonstrate the detection of reaction heats for a model reaction - the catalytic oxidation of propane in air. The reaction was performed over a thin film Pd catalyst at concentrations of propane ( 0.01 to $0.8 \mathrm{vol} \%$ ) which is significantly below lower explosion limit of propane. The sensor exhibits an immediate and reversible response upon exposure to propane in air. The short response time allows this detection mechanism to be applied in transient studies of chemical reactions, such as differential scanning microcalorimetry.

The sensor can be applied for the detection of reaction heats and kinetic analysis, where commonly higher concentrations of reactive components are used, which will lead to greater heat release. Besides that it can be utilized as a safety sensor for the detection of propane leaks. In addition, immobilization of a highly active catalyst with a high surface area on the sensor can significantly increase the sensitivity. 
Thin films of titanium silicide $\left(\mathrm{TiSi}_{2}\right)$ formed on heavily boron doped polycrystalline silicon (poly-Si/B ${ }^{+}$) were applied for resistive temperature sensing. The TCR and the thermal stability were tested on $\mathrm{TiSi}_{2} / \mathrm{poly}$-Si thin films on $\mathrm{Si}$ and FS substrates and on $\mathrm{TiSi}_{2} /$ poly-Si resistors suspended on a SiRN membrane. The TCR for flat, non-patterned layers was found to be $(2.9-3.1) \times 10^{-3} /{ }^{\circ} \mathrm{C}$ in the range of temperatures from 20 to $800{ }^{\circ} \mathrm{C}$. The TCR of suspended TiSi $/ 2$ poly-Si resistors was measured $(3.6-4) \times 10^{-3} /{ }^{\circ} \mathrm{C}$ from 20 to $185^{\circ} \mathrm{C}$.

The process developed for poly-si and $\mathrm{TiSi}_{2} /$ poly-Si resistive thin films was implemented in a microhotplate for application in microcalorimetric sensing. Thermo-electrical characterization and analysis was performed. The sensor consumes ca. $12 \mathrm{~mW}$ at an operational temperature of $350{ }^{\circ} \mathrm{C}$. Thermal imaging with an infrared camera indicated heating in the high-ohmic part with the temperature gradient along the active sensor area estimated to be $4{ }^{\circ} \mathrm{C} / \mu \mathrm{m}\left(\right.$ at ca. $\left.500{ }^{\circ} \mathrm{C}\right)$. The heating and cooling times were found to be ca. $1 \mathrm{~ms}$ and $8 \mathrm{~ms}$. The power vs temperature curve was determined while operating in both stationary and constant flow conditions and shows the thermal resistance to ambient of ca. $28{ }^{\circ} \mathrm{C} / \mathrm{mW}$. We demonstrate the detection principle in constant temperature mode based on model reactions of propane and hydrogen oxidation in air at temperatures $200-350{ }^{\circ} \mathrm{C}$ and $100{ }^{\circ} \mathrm{C}$, respectively.

Sputtered thin films and sol-gel based catalyst were investigated as potential functional coatings in micromachined gas sensors. Sputtered thin films are characterized by a low surface area and, if there is a non-active phase present on the top layer, low activity. However, introducing a micropattern may resolve this issue. The catalyst does not require any specific activation procedure and can be used directly. Sputtered thin films can be deposited and patterned in a straightforward way using magnetron sputtering in combination with metal lift-off and photolithography.

Sol-gel based supports ensure a high surface area and a high dispersion, resulting in high activity. Sol-gel based catalyst precursors require the development of specific immobilization techniques in combination with activation procedures (calcination, reduction). Soft-lithographic techniques were applied for the deposition of a boehmite sol.

In terms of reproducibility and efficiency: lithography-assisted self-assembly and MIMIC are considered as the most promising techniques to pattern a sol-gel based catalyst.

Both the metal thin films as well as the metal dispersed sol-gel based $\gamma$-alumina layers were characterized and showed good catalytic activity.

A novel heating and temperature sensing concept proposed by Chair of Semiconductor Components (University of Twente) has been utilized for development of ultra-low-power hot surface devices. A so-called "link" heating and sensing device is based on a small and highly conductive link which can act both as heater and temperature sensor. This is a novel type of heater where the dissipation of heat takes place inside the link acting as a resistor. In such a structure the electrical and thermal resistances are controlled independently. This reduces losses from conduction via the resistive leads, which is a practical problem for all type of heaters, as the leads remain "cold" during the device operation. This increases the overall efficiency of the heating device. These ultra low power, CMOS compatible, suspended membrane, resistive sensors and actuators with operating temperatures up to $300^{\circ} \mathrm{C}$ were tested as transducers for calorimetric sensing applications.

The sensors consist of a thin film, titanium nitride resistive element in a 4-point configuration: meander-, spiral-, linkshaped heaters. They can facilitate local heating and temperature sensing of $0.001-0.1 \mathrm{~mm}^{2}$ area at a power consumption less than $3 \mathrm{~mW}$ and for particular designs even limited to few $\mu \mathrm{W}$. This enables potential applications in various portable sensing systems where rapid temperature sensing and/or thermal actuation with high spacial resolution and minimized power consumption are required.

The feasibility of successful operation was demonstrated with two examples: flow meter and thermal conductivity detector. It was not possible to obtain reliable measurements while operating devices as a pellistor. 


\section{Samenvatting}

In het "Hot Silicon" project zijn laag- en ultra-laag-vermogen-meetinstrumenten ontwikkeld, die gebaseerd zijn op siliciumtechnologie. De ontwikkelde geminiaturiseerde thermische sensoren en actuatoren maken gebruik van een heet oppervlak, en kunnen worden toegepast als katalytische gassensoren en als micro- of nanocalorimeters. Wetenschappelijke en technologische aspecten die in het onderzoek ter sprake zijn gekomen zijn:

- Ontwerp en fabricage van ultra-laag-vermogen op silicium gebaseerde thermische sensoren en actuatoren met een meerzijdig ingeklemd membraan met een heet oppervlak;

- Integratie van de sensoren en actuatoren in een microkanaal met gasstroom, en onderzoek naar de relevante microfluidische aspecten zoals stromingsgedrag en -controle, warmtetransport, en transport van reactieve gassen;

- Onderzoek naar mogelijke toepassingen op het gebied van microcalorimetrische metingen, zoals het bestuderen van thermisch geactiveerde fysische en chemische processen op een chip, waaronder de bepaling van reactiewarmte en adsorptie/desorptiekinetiek.

Er is een numeriek model ontwikkeld dat het katalytische microgassensorsysteem beschrijft dat bestaat uit een microverwarmingsplaat op verhoogde temperatuur waarop geïmmobiliseerd een reactieve katalysator. De invloed van de ontwerpparameters voor de microverwarmingsplaat, zoals dikte en materiaalkeuze van het isolerende membraan, dimensies van de geëtste holte in het silicium substraat, en het type substraat, op vermogensverbruik, totale thermische efficiëntie, warmteverlies en oppervlaktetemperatuursverdeling, is onderzocht. Het materiaal gekozen voor het membraan beïnvloedt het warmteverlies meer dan de dikte van het membraan, als alle andere parameters constant worden gehouden. De implementatie van dunnere membranen of van membranen uit materialen met een lagere thermische geleidbaarheid leidt tot een afname in het totale vermogensverbruik, maar helaas ook tot een niet uniforme oppervlaktetemperatuursverdeling. Wanneer de verhouding tussen verhit oppervlak en membraanoppervlak, $\mathrm{a}_{h} / \mathrm{a}_{m}$, onder de 0.25 wordt gekozen, wordt de warmteflux naar het omringende substraat gereduceerd, en ontstaan er geen sterke gradiënten in het oppervlaktetemperatuursprofiel. De belangrijkste paden voor warmteverlies zijn via het Si substraat (ca. $40 \%$ ) en via de lucht (ca. $60 \%$ ), de hoeveelheid warmte die verloren gaat door het membraan is minder dan $1 \%$ van het totale toegevoerde vermogen. Echter, het membraan introduceert wel een warmtelek naar het $\mathrm{Si}$, daarom moeten de dikte, en vooral het materiaal van het membraan adequaat gekozen worden, afhankelijk van de vermogensverbruikseisen die gelden voor een specifiek toepassing.

Het warmte-effect van een chemische gasreactie is evenredig met de concentratie van het te meten gas (propaan) in lucht, en de van de aangelegde basistemperatuur. Als de gekozen katalysator niet actief genoeg is, dienen hogere temperaturen te worden toegepast ter compensatie. Stilstaande (geen gasstroming) condities hebben de voorkeur omdat daarbij de verliezen minder zijn in vergelijking met bedrijven van het systeem bij constante stroming. Uit actuatieoogpunt heeft het weinig zin om de dimensies van het verwarmingselement verder te verkleinen tot het nanometerbereik, indien men daarbij de grootte van het katalysatorelement constant houdt. Een kleinere verhitter zal niet in staat zijn om voldoende vermogen te leveren om het katalysatorvolume tot de gewenste (de ontstekings-) temperatuur te verhitten. Een katalysator veel kleiner dan het verwarmingselement is ook onwenselijk, omdat in dat geval de overbodige warmte slechts verspild wordt door verhitting van de omringende lucht. Ideaal is derhalve een ontwerp waarin de verhitter en de katalysator van vergelijkbare dimensies zijn, ofwel $\mathrm{a}_{\text {cat }} / \mathrm{a}_{h}$ beneden de 2.5. Een klein weerstandselement geïntegreerd met een verhitter met sterk vermogen kan dan dienen als een temperatuursensor.

De fabricage, het karakteriseren en het testen van een goedkope, laagvermogen, CMOS-compatibele micro-calorimetrische sensor voor de detectie van reactiewarmte wordt uitvoerig beschreven. De constructie van het calorimetrische sensorsysteem gebaseerd op extrinsiek-gedoteerde polysilicium resistieve verwarmingselement en en temperatuursensoren is gedemonstreerd. Zowel de compatibiliteit met Si-technologie als de chemische inertheid bij verhoogde temperaturen maakt polysilicium attractief voor de integratie in microreactoren en sensoren. Evenwel, het niet -lineaire gedrag van de weerstand en de drift blijven uitdagingen die een succesvolle implementatie als functioneel sensormateriaal belemmeren. De detectie van reactiewarmte is gedemonstreerd voor een model reactie - de katalytische oxidatie van propaan in lucht. 
De reactie werd uitgevoerd over een dunne film Pd katalysator bij propaanconcentraties van 0.01 tot 0.8 vol \%, wat significant lager is dan de laagste explosielimiet van propaan, en daarom een zeer relevant gebied voor gaslekdetectie.

De sensor vertoont een onmiddellijke reversibele respons op blootstelling aan propaan in lucht. De snelle responstijd laat het toe om het detectiemechanisme toe te passen in transient studies van chemische reacties, zoals in differentiële scanning (micro)calorimetrie (DSC). De sensor kan derhalve worden toegepast voor de bepaling van reactiewarmtes en kinetische analyses, waarvoor normaalgesproken hoge concentraties aan reactieve componenten worden gebruikt, met een navenant grote warmteontwikkeling. De sensor kan derhalve ook worden gebruikt als veiligheidsmonitor voor propaanlekkage. Het aanbrengen van een nog actievere katalysator met een groter oppervlak kan de gevoeligheid nog significant verbeteren.

Dunne films van titanium silicide $\left(\mathrm{TiSi}_{2}\right)$ gevormd op zwaar boor-gedoteerd polykristallijn silicium $\left(\mathrm{poly-Si} / \mathrm{B}^{+}\right.$) zijn toegepast in resistieve temperatuurmeting. De TCR (temperatuurcoëfficiënt van de weerstand) en de thermische stabiliteit zijn getest aan $\mathrm{TiSi}_{2} /$ poly-Si dunne films op Si en op kwartsglas (fused silica) substraten, en aan TiSi $2 /$ poly-Si weerstanden op een SiRN (silicium-rijk siliciumnitride) membraan. De TCR van vlakke, niet gepatroneerde lagen was $(2.9-3.1) \times 10^{-3} /{ }^{\circ} \mathrm{C}$ voor het temperatuurgebied van 20 tot $800{ }^{\circ} \mathrm{C}$. De TCR van de $\mathrm{TiSi}_{2} /$ poly-Si weerstanden op het membraan bedroeg $(3.6-4) \times 10^{-3} /{ }^{\circ} \mathrm{C}$, voor 20 tot $185^{\circ} \mathrm{C}$. Het proces ontwikkeld voor poly-Si en $\mathrm{TiSi}{ }_{2} /$ polySi resistieve dunne films is gecombineerd met de fabricage van een microverwarmingselement (een microhotplate) met beoogde toepassingen in microcalorimetrische metingen. Een thermo-elektrische karakterisering en analyse van de resulterende devices werd uitgevoerd, waaruit bleek dat de sensoren ca. $12 \mathrm{~mW}$ consumeren bij een operationele temperatuur van $350^{\circ} \mathrm{C}$. Thermische imaging met een infrarood camera liet opwarming zien in het hoog-Ohmige deel van de sensor. Tevens kon hieruit geschat worden dat de temperatuurgradiënt langs het actieve sensorgebied $4{ }^{\circ} \mathrm{C} / \mu \mathrm{m}$ is $($ at $\mathrm{ca}$. 500 ${ }^{\circ} \mathrm{C}$ ). De opwarm- en afkoeltijden werden gemeten als ca. $1 \mathrm{~ms}$ en $8 \mathrm{~ms}$. Een vermogen vs. temperatuur curve werd bepaald voor zowel stationaire als constante gasstroomcondities, waaruit een thermische weerstand naar de omgeving werd gevonden van ca. $28^{\circ} \mathrm{C} / \mathrm{mW}$. Het detectieprincipe werd gedemonstreerd in een constante-temperatuur-modus, voor de modelreacties propaan oxidatie en waterstofgas oxidatie in lucht bij temperaturen van $200-350{ }^{\circ} \mathrm{C}$ en $100{ }^{\circ} \mathrm{C}$, respectievelijk.

Gesputterde dunne films en sol-gel-gebaseerde katalysatorlagen werden onderzocht als potentiele functionele coatings in micromachined gassensoren. Gesputterde dunne films worden getypeerd door een laag oppervlak en, indien er een niet-actieve toplaag aanwezig is, een lage activiteit. Het introduceren van een micropatroon zou dit laatste bezwaar kunnen opheffen. De katalysator heeft geen specifieke activeringsprocedure nodig en kan direct worden gebruikt. Gesputterde dunne films kunnen worden aangebracht en gepatroneerd op eenvoudige wijze, via magnetron sputtering in combinatie met lift-off fotolithografie. Op sol-gel gebaseerde onderlagen garanderen een hoog oppervlak en grote dispersie, wat resulteert in hoge activiteit. De sol-gel katalysatorreactanten vereisen evenwel de ontwikkeling van specifieke immobilisatietechnieken in combinatie met activeringsprocedures zoals calcinatie en reductie. Soft-lithografische technieken werden toegepast voor de depositie van Boehmite sol. In termen van reproduceerbaarheid en efficiëntie kunnen de op lithografie gebaseerde zelf-assemblage en de zogenaamde MIMIC techniek worden beschouwd als de meest belovende technieken om een sol-gel katalysator te patroneren. Zowel de dunne metaal films als de sol-gel gebaseerde -alumina lagen met metaaldispersie werden gekarakteriseerd en vertoonden een goede katalytische activiteit.

Een nieuw verhittings- en temperatuurmeetconcept voorgesteld in de leerstoel Semiconductor Components werd onderzocht voor gebruik in ultra-laag-vermogen "hot surface" devices. Deze nano-"link" is toe te passen als verwarmings- en als sensorelement, is gebaseerd op een zeer kleine geleidende verbinding, waarin de link met zijn hoge weerstand als verhitter optreedt door sterke lokale warmtedissipatie. In deze structuur zijn de elektrische en de thermische weerstanden onafhankelijke van elkaar gecontroleerd, waardoor verliezen door conductie via de resistieve verbindingen, een praktisch probleem voor alle typen verwarmingselementen, worden gereduceerd omdat deze verbindingen koud blijven gedurende deviceoperatie. Hiermee neemt de efficiëntie van het verwarmingselement toe. Deze ultra-laag-vermogen, CMOS compatibele, membraan-geïntegreerde, resistieve sensoren en actuatoren met werktemperaturen tot $300{ }^{\circ} \mathrm{C}$ werden getest als omzetter in calorimetrische sensor applicaties. De sensoren bestaan uit dunne film titaniumnitride resistieve elementen in een 4-puntsconfiguratie, met verschillende ontwerpen, namelijk meander, spiraal, en eenvoudige link-vormige verhitters. Ze faciliteren lokale verhitting en temperatuurbepaling op een oppervlak van $0.001-0.1 \mathrm{~mm}^{2}$ met een vermogensverbruik van minder dan $3 \mathrm{~mW}$, voor sommige ontwerpen zelfs gelimiteerd tot een paar $\mu \mathrm{W}$. Dit staat het toe te denken aan toepassingen in mobiele sensorsystemen waarin snelle temperatuurmeting en/of thermische actuatie met een hoge plaatsresolutie en minimaal vermogensverbruik gewenst zijn. De toepasbaarheid van het concept werd succesvol gedemonstreerd met twee voorbeelden: een stromingssensor en een thermische geleidbaarheidssensor. Betrouwbare metingen met het nanolink-concept verwerkt in een pellistor zijn helaas nog niet succesvol geweest. 


\section{List of Publications}

\section{Conference contributions}

2008

- E.Vereshchagina, J.G.E. Gardeniers. Low-power hot-surface silicon-based devices for chemical sensors and actuators. Ameland Summer School, Ameland (The Netherlands) Poster

2009

- E.Vereshchagina, J.G.E. Gardeniers. Downscaling of temperature sensors and actuators for chemical analysis ona-chip. Proceedings of the Sense of Contact 11, Zeist 24-26 March (The Netherlands) Oral

- E.Vereshchagina, J.G.E. Gardeniers. Development of hot-surface polysilicon-based chemical sensor and actuator with integrated catalytic micropatterns for gas sensing applications. AIP Proceedings of the 13th International Symposium on Olfaction and Electronic Nose, Brescia (Italy), 15-17 April, 1137 407-408 Poster

2010

- E.Vereshchagina, R.A.M. Wolters, J.G.E. Gardeniers. Development and characterization of titanium disilicide thin films for high temperature gas sensing applications, Proceedings of the European Material Research Society Conference, Strasbourg (France) 9-10 June Oral

- E.Vereshchagina, J.G.E. Gardeniers. Development of a CMOS compatible microcalorimetric sensor for the selective detection and kinetic analysis of propane in air. Proceedings of the $5^{\text {th }}$ Asia-Pacific Conference on Transducers and Micro-Nano Technology, Perth 6-9 July (Western Australia) Oral

- E.Vereshchagina, J.G.E. Gardeniers. Microcalorimetric detection of propane, propene and propyne using a CMOSbased microhotplate sensor. Proceedings of the $13^{\text {th }}$ International Meeting on Chemical Sensors, Perth 11-14 July (Western Australia) Oral

- E.Vereshchagina, R.A.M. Wolters, J.G.E. Gardeniers. Development of titanium silicide - boron doped polysilicon resistive temperature sensors. Proceedings of the the Mesa+ Annual Meeting, Enschede 14 September (The Netherlands) Poster

2011

- E.Vereshchagina, R.A.M. Wolters, J.G.E. Gardeniers. Chemical analysis and sensing using CMOS microcalorimetric sensors and actuators. $9^{\text {th }}$ meeting of Electrochemical Society. Electrochemical Sensors: From nanoscale engineering to industrial applications. Turku 8-11 May (Finland) Poster

\section{Journal Publications}

- O.Bliznyuk, E.Vereshchagina, E.Stefan Kooij, B.Poolsema. (2009) Scaling of anisotropic droplet shapes on chemically stripe-patterned surfaces. Physical Review E 79(4) 041601.

- E.Vereshchagina, J.G.E. Gardeniers. (2011) Measurement of reaction heats using a polysilicon-based microcalorimetric sensor. Sens. Actuators A. 169(2) 308-316.

- E.Vereshchagina, R.W.A. Wolters, J.G.E. Gardeniers. (2011) Development of titanium silicide - boron doped polysilicon resistive temperature sensors. J. Micromech. Microeng. 21105022 (14pp).

- E.Vereshchagina, O.Bliznyuk, K.Altena-Schildkamp, R.M.Tiggelaar, J.G.E.Gardeniers (2011) Selection, characterization and local deposition of catalytic thin films. J. Michromech. Microeng. status: submitted.

- E.Vereshchagina, R.M.Tiggelaar, R.G.P. Sanders, J.G.E.Gardeniers, Characterization of $\mathrm{TiSi}_{2} / \mathrm{Poly}-\mathrm{Si}$ micro hotplates for analysis of gaseous samples. Sens. Actuators B status: in preparation. 



\section{Acknowledgments}

Finally!! I arrived to this point alive! Here comes the part of the thesis that will be read by the biggest amount of people!

These four years in Enschede was an amazing time for me and here I would like to thank all people whom I had a pleasure to work with, whom I met in one or other way during this time for helping me going through this process.

I would like to thank Prof. J.G.E. Gardeniers for giving me the opportunity to work in such a productive environment as MCS group. Thank you, Han, for your very interesting way of guidance, for all the freedom to do best and worst out of my work and ideas and to learn on my own mistakes. It was not easy for me from time to time, but I think I learned a lot from this experience and I never regretted I challenged myself for this project. I'm really grateful for everything I was given the opportunity to do and learn in your group.

I would like to thank the "Hot Silicon" project team for being an enthusiastic working environment and setting high standards for research. It was a great pleasure to be part of the team, I appreciate all your support during my research.

Alexey Kovalgin, thank you for all your encouragement and (sometimes too) creative ideas, for showing what does it mean to be a real scientist looking for great challenges. Thank you for your constructive criticism and assistance in making my thesis better. I really appreciate all that.

Alfons, it was great pleasure to work with you! You will be always for me one of the best examples of super-organized perfectionists! Thank you for always being there to help and have patience towards my sometimes chaotic way of doing research.

I would like to thank Prof. R.A.M. Wolters for his help and guidance in my research. Thank you, Rob, for always being supportive and understanding, without your help and advices it simply would not be possible to accomplish this work. It has been a great honor for me to have this opportunity to work with you.

I want to thank the members of my promotion committee, Prof. K. Seshan, Dr. A.G. van den Bos, Prof.dr.ing. A.J.H.M. Rijnders, Prof.dr.R.Dekker for reading my thesis and for your impact on the final version, your suggestions were very useful, thank you for helping me to improve the manuscript.

During these four years what I probably enjoyed the most is clean room work, but this experience would be not the same without combined effort of all clean room staff, 
users and technicians from other groups. Thank you all.

During my PhD I was lucky to work in one of the best groups in UTwente, not only in terms of research and work quality but also from the point of view of working environment and people. Thank you all for being with me and helping me in a different way!

There is one more person without whom I would not make it up to here. Roald, thank you very much for all your advices, energy, time, for correcting my thesis, trying to find a single logical thing in what I was writing, for keeping up my spirit, without your suggestions this thesis surely would not be the same. You are the best!

Of course it is not possible to get anything done without a help and knowledge of the technicians. In my case I was lucky, as Stefan "has a lot of knowledge" which helps you to kill a lot of waiting time in the clean room. Now I'm thinking, did I actually learn anything from you?.. ah..sure, I learned about karting, movie making, weirdest youtubes...Thanks for everything, Stefan, you are definitely "the pleasant one" to work with!)))

Jacqueline, the secretary of MCS, thank you very much for being such a nice and helpful person. Your support and help in organization in last days was very important.

I'd like to thank Regina Luttge for all advices. I always will remember you as very energetic person with whom I can discuss anything. Thank you for encouraging me to persuade my career opportunities.

I want to thank my officemates - Anil, Kevin, Shreenath, Svetlana, Sertan - for helping me and making every day working life nice. Svetlana, thank you for listening and sharing your opinion, for supporting me especially on the stage of writing of my thesis. Sertan, the "compiler", thanks for your kind advices and honest way of expressing your opinion. Your humor and easy way of taking things was really helpful, may be one day I could learn to deal with the troubles at work as calm as you..hmm...or may be not!

Wojtek, it was always interesting to find how well you understand some of my EastEuropean mentality, thank you for nice discussions and various time you helped me with advices during my PhD...not sure I should thank you for introducing me to the world of Red Bull addiction!

The best bodyguards - David and Nikolay - thanks for agreeing to help me this day! Piotr, Machek, Brigitte, Takayuki, Engin, Wim, Arturo, Bilge, Reyes, thank you!!!

During my PhD I also worked in other labs. CPM-ers, thanks for hosting me in your very organized and clean lab, it was a great pleasure to introduce some degree of chaos there from time to time! Special thank to Bert and Karin for their help.

I'd like to acknowledge my collaborators - Olesya, it was always a pleasure to dis- 
cuss things with you, you have such an amazingly broad knowledge in physics! I have been fortunate to come across many good people who became friends of mine. I would like to thank Julian, David, Vincent, Marijana, Inga, Shilpii, Gacia, Arturo, Olga(-s), Chilangos for great parties, musical entertainment, your energy and positive spirit!!!

David, we became "enemies" since the first day we met, hope we are going to keep it this way!

Julian, dear friend with big heart!! thank you very much for all your support, understanding, especially last months of my PhD, for teaching me salsa, Usselerweg dinners with enjoyable chats and for your delicious Mexican food!!

Shilpii, thank you for always being cheerful person, for teaching me secrets of Indian food (I can not eat without coriander anything now!), for all conversations and also for allowing me to practice my image-making skills on you during our parties!

Maki, Inga, our great train trip to Lausanne and back, accidentally taking too long was very memorable!..and I think we all can still recall)))...we should repeat it definitely!

Arturo, pinche de la chingada(??), my favorite salsa dancer (may be after Julian and David, haha), thanks for being such a nice person, and your enchiladas were also super tasty!))))

Gacia, thanks for organizing girls nights and movies activities. I still don't understand why our masterpiece about "animals in prisons" so geniously performed by Chris was not awarded for Oscar yet?!

Hippocampus members, friends I met there and all Horstlinde horses are thanked for opportunity to learn riding and share nice time during the lessons and at the bar (you can figure out very fast why you need the bar at the manege!)! Also, Pro Deo International and other student clubs I've been involved shortly, thanks, for making Enschede life more colorful!

There are so many people I'd like to thank, that it could be I forget to name somebody, well sorry about that! I'm thankful to all people I have met! I will miss you all! After the graduation we all scatter somewhere on this planet, who knows where we meet next time, may be in Enschede? Dublin? Saint-Petersburg? or somewhere else.. Deep from my heart I wish you all the best in your life!

Last but not least, I would like to thank my family and friends for their love and support during my stay in Enschede, and my boyfriend Roman for his love, patience and enormous support.

Liza. 



\section{Author biography}

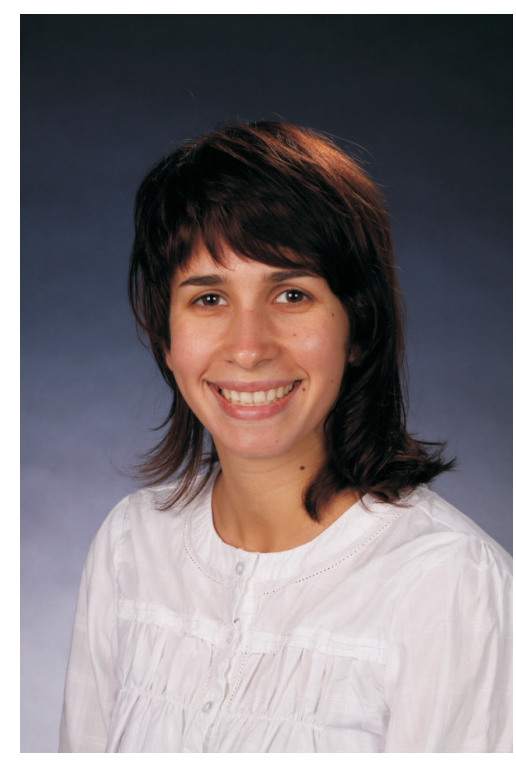

Elizaveta Vereshchagina was born in Saint-Petersburg, Russia, on May 16, 1984. She received her Engineer degree in Microelectronics and Solid State Components of Electronics from the Saint-Petersburg State Electrotechnical University LETI (St. Petersburg, Russia, 2007) and the M.Sc. degree in Technical Physics from the Lappeenranta University of Technology (Lappeenranta, Finland) in 2007. From September 2007 to 2011 she did her Ph.D. at the Chair of Mesoscale Chemical Systems, MESA+ Institute for Nanotechnology, University of Twente (Enschede, The Netherlands) under supervision of Prof. dr. J.G.E. Gardeniers on the development of low power hot surface silicon devices for chemical sensors and actuators. In September 2011 she joined the Microfluidic Platforms group, Dublin City University (Dublin, Ireland) as a post-doctoral researcher and is currently working on the development of point-ofcare diagnostic devices under supervision of Prof. J. Ducrée. 